\title{
Deciphering the Bifunctional Role of Disulfide Oxidoreductase DsbA2 in
} Legionella pneumophila

by

Zegbeh Zekie Kpadeh

\begin{abstract}
A Dissertation presented to the Graduate Faculty of the University of Virginia in Candidacy for the Degree of Doctor of Philosophy

Department of Microbiology, Immunology \& Cancer Biology
\end{abstract}

at

University of Virginia School of Medicine April 2014

(C) Copyright by Zegbeh Z. Kpadeh, 2014 


\title{
Dedication
}

This dissertation is dedicated in loving memory to my beloved brother, Massaquoi M. Kamara Jr.; who taught me that a fulfilled life is a life of service. Thanks for showing me what happiness, loyalty, and love look like. May you rest in blessed peace!

\section{$\mathscr{8}$}

My dearest mother, Argbah K. Momolu; your life has provided me with a wonderful, day-byday, lifetime demonstration of a godly mother who understands the true value of family, hard work, dedication, humility, giving and higher education.

\begin{abstract}
$\mathscr{8}$
My amazing father, Selma D. Kpadeh; thanks for your encouragement and belief in the importance of me being a well-rounded lady in life.
\end{abstract}

My wise and loving sister, Solieh G. Kpadeh; you have been my motivator and silent role model. Thank you for always being very proud of me, and for encouraging me and praying with me to see my dream fulfilled. 


\section{Table of Contents}

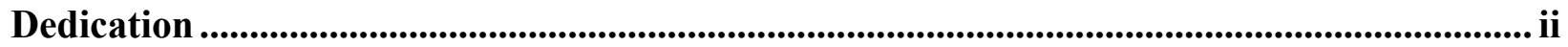

Table of Contents ................................................................................................................ ii

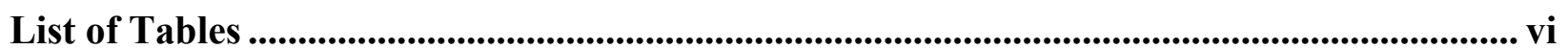

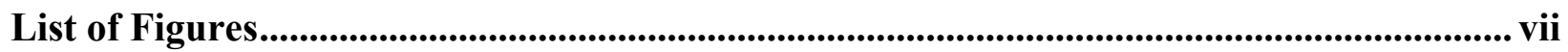

List of Abbreviations and Symbols ....................................................................................... ix

Acknowledgements ................................................................................................................................. xiii

Abstract........................................................................................................................................ xiv

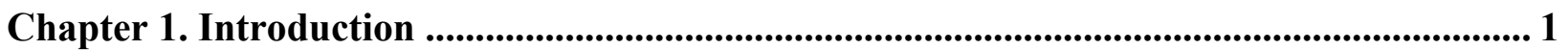

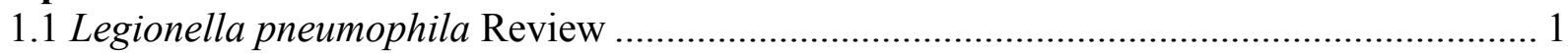

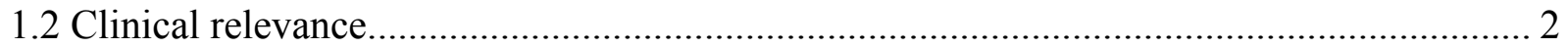

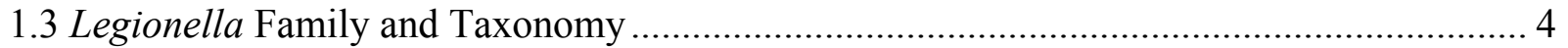

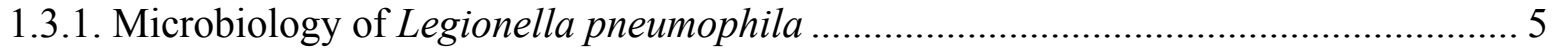

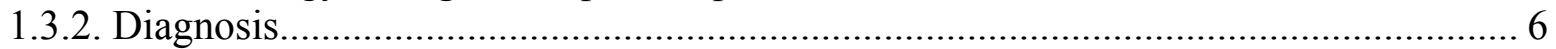

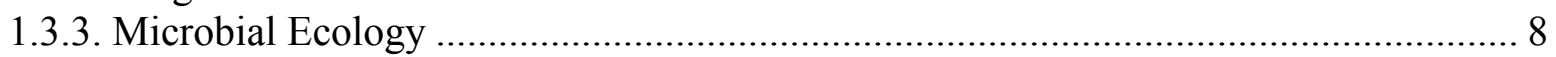

1.3.4. The Developmental cycle of Legionella ……......................................................... 9

1.4 Virulence of Legionella in protozoa and Human hosts .................................................... 13

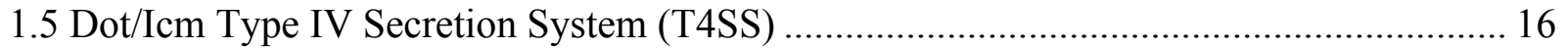

1.6 Protein Folding and Disulfide Bond Formation................................................................ 17

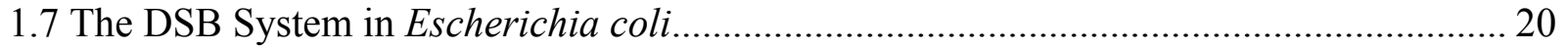

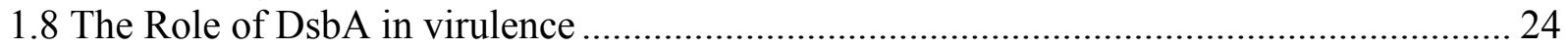

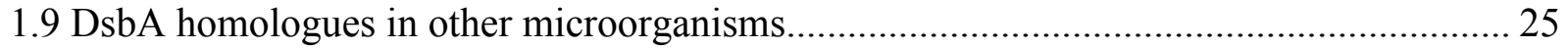

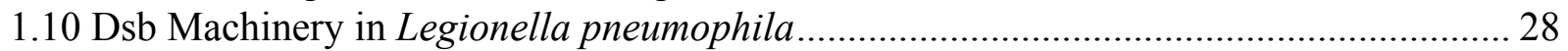

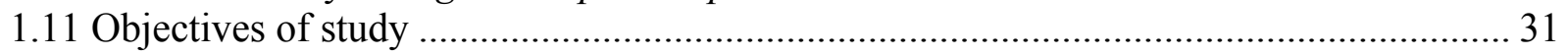

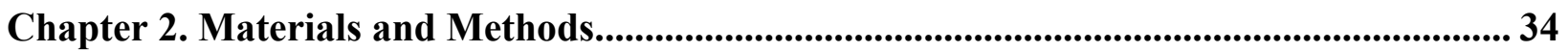

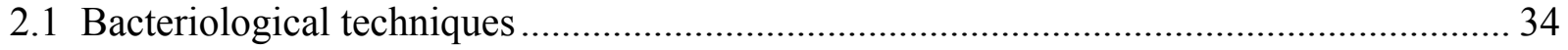

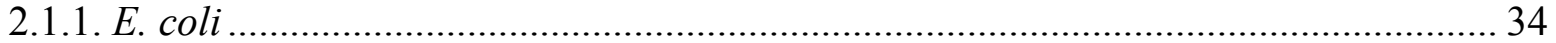

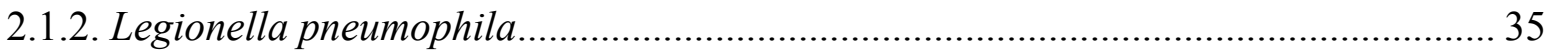

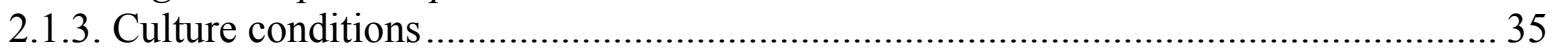

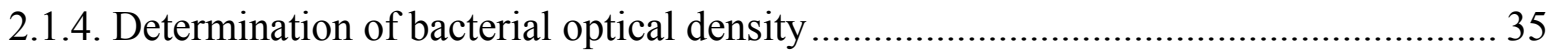

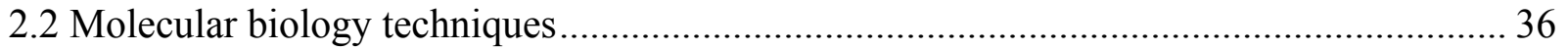

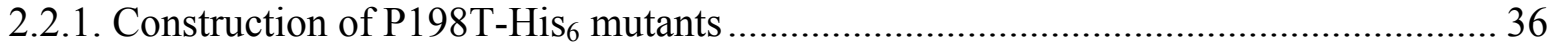

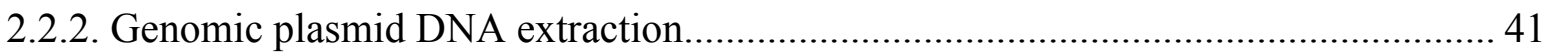

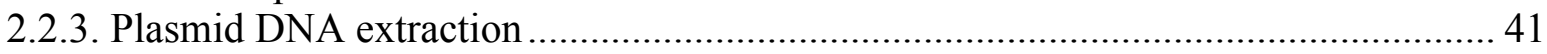

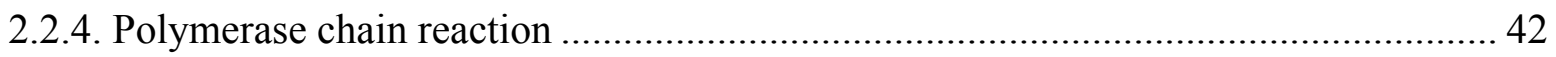

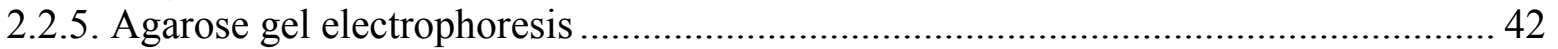

2.2.6. Purification of DNA from agarose gels .................................................................. 43

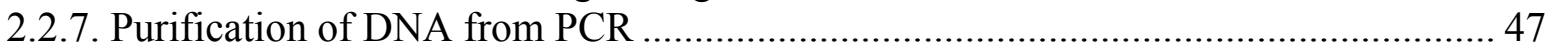

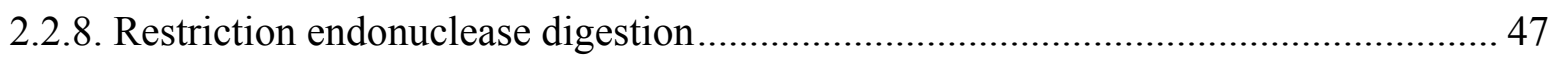

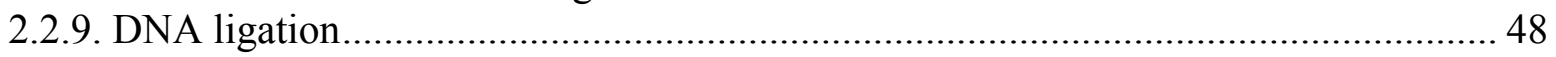

2.2.10. Preparation of electrocompetent cells ...................................................................... 48 
2.2.11. Electroporation of plasmids into E. coli or L. pneumophila .................................. 49

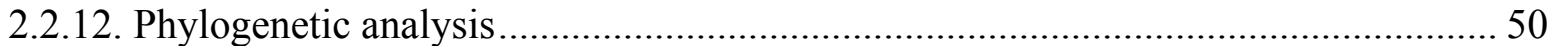

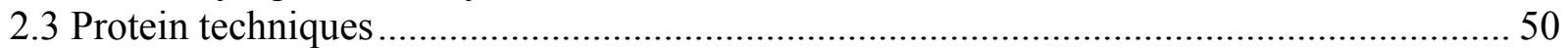

2.3.1. Sodium Dodecyl Sulfate-Polyacrylamide Gel Electrophoresis (SDS-PAGE) ............ 50

2.3.2 Diagonal gel electrophoresis of DsbA1 cellular extracts............................................. 51

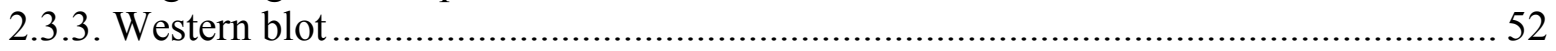

2.3.4. Isolation of native DsbA2 by osmotic shock ........................................................... 52

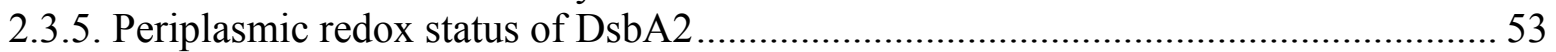

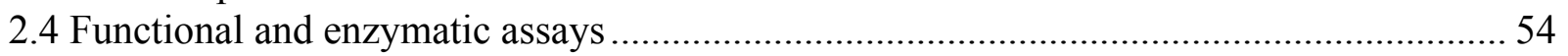

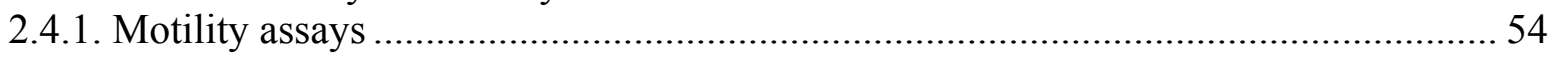

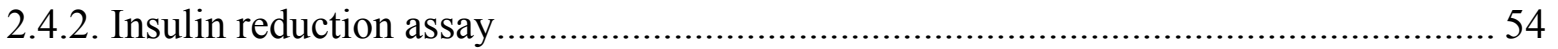

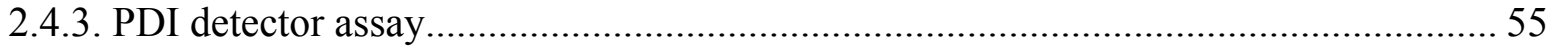

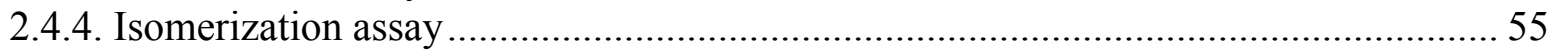

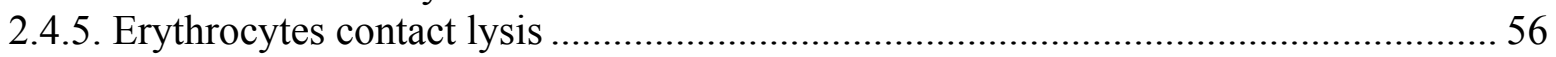

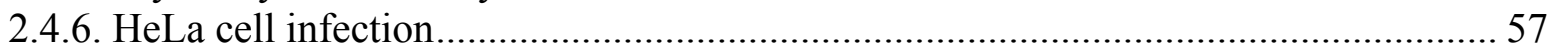

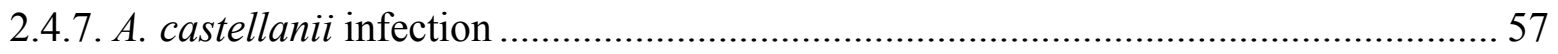

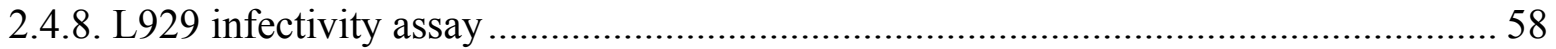

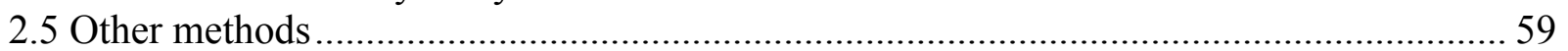

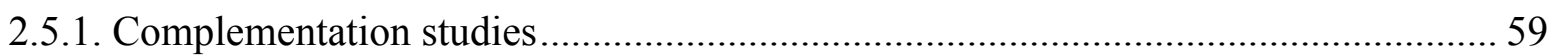

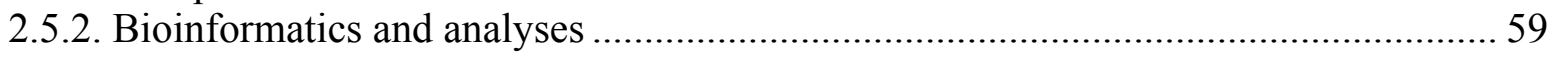

2.5.3. Phylogenetic analysis of DsbA1, DsbA2 and EcDsbC............................................. 59

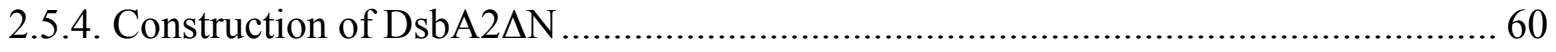

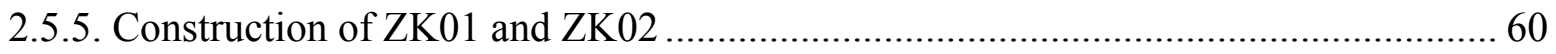

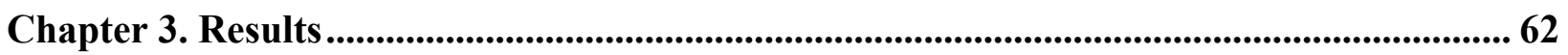

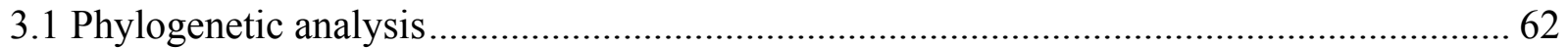

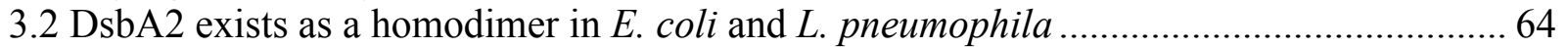

3.3 Lpn $d s b A 1$ and dsbA2 $\Delta \mathrm{N}$, but not $d s b A 2$ restore motility to Ec $d s b A$ mutant ................... 66

3.4 Periplasmic redox status of monomeric and homodimer DsbA2 in E. coli ....................... 69

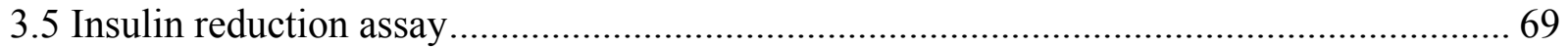

3.6 Legionella pneumophila DsbA2, but not DsbA1 or DsbA2 $\Delta \mathrm{N}$, exhibits PDI activity....... 71

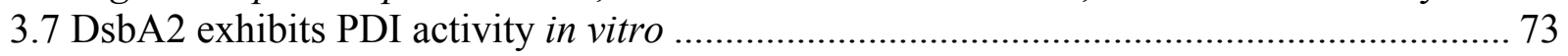

3.8 Identification and nomenclature of DsbB and DsbD proteins .......................................... 76

3.9 Evaluation of DsbB1 and DsbB2 in E. coli dsbB mutants.............................................. 80

3.10 Both Lpn DsbB1 and DsbB2 interact with monomeric DsbA2 (DsbA2 $\Delta \mathrm{N}$ ) in E. coli ... 80

3.11 Lpn DsbD1 and Lpn DsbD2 are unique for DsbA2 bifunctional activity ......................... 82

3.12 DsbA2 exhibits oxidase activity in the presence of Lpn $d s b B$ s and Lpn $d s b D$ s............... 86

3.13 DsbA2 mutant constructs have no growth or expression defect in vitro .......................... 90

3.14 Monomeric DsbA2 mutant exhibits a defect in attachment, invasion and intracellular

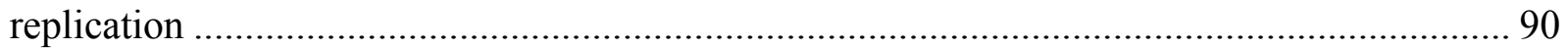

3.15 Functional Dot/Icm T4SS assessment of monomeric DsbA2 activity............................ 95

3.16 The importance of DsbA1 for L. pneumophila pathogenesis .......................................... 97

3.17 Novel phenotype: $d s b A l$ knockout exhibits a defect in pigment production .................. 101

$3.18 d s b A l$ knockout exhibits a hypervirulent phenotype in plaque assay .............................. 101

3.19 DsbA1 is a modulator of virulence in Legionella pneumophila .................................... 103

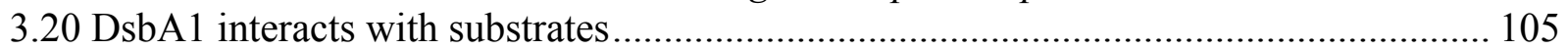

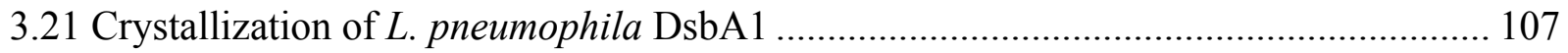




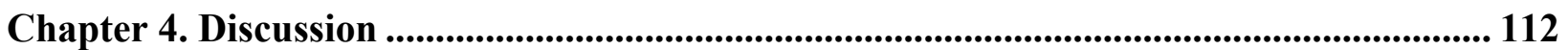

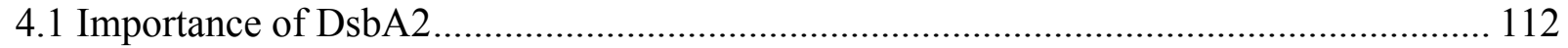

4.2 DsbA2 of L. pneumophila differs from canonical DsbA of E. coli ................................ 115

4.3 How are the redox forms maintained? ....................................................................... 116

4.4 Functional activity of homodimeric DsbA2 ............................................................ 118

4.5 Reconstitution of L. pneumophila $d s b$ genes in E. coli background............................... 120

4.6 In vivo characterization of DsbA2 forms for virulence in L. pneumophila .................... 126

4.7 Why is DsbA1 maintained in L. pneumophila? ...................................................... 128

Chapter 5. Conclusion ........................................................................................ 130

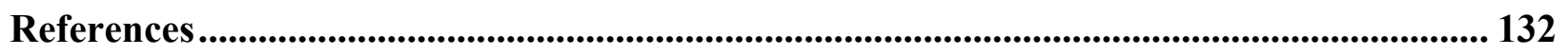




\section{List of Tables}

Table 1. Disulfide bond composition of genera expressing DsbA2 ....................................... 30

Table 2. Bacterial strains and plasmids used in this study..................................................... 37

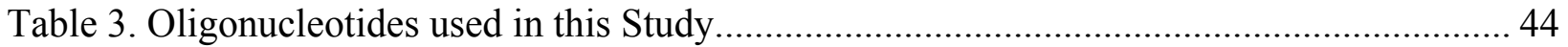




\section{List of Figures}

Figure 1. Simplified model illustrating the intracellular and extracellular growth cycles of $L$.

pneumophila... 10

Figure 2. Morphological differences between the two principal forms of L. pneumophila in vivo

Figure 3. The type IVB secretion systems of Legionella pneumophila .................................... 15

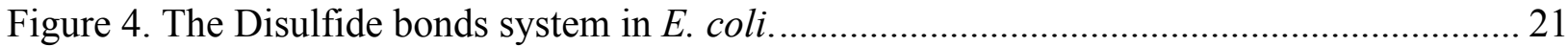

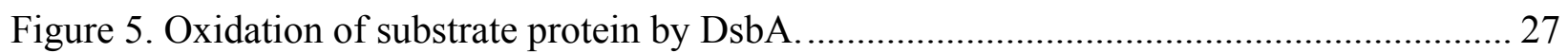

Figure 6. Dimerization domain and secondary structure comparison among DsbA proteins of

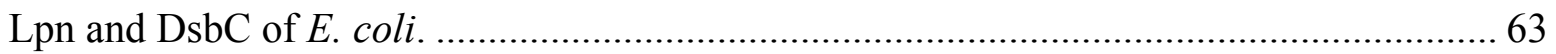

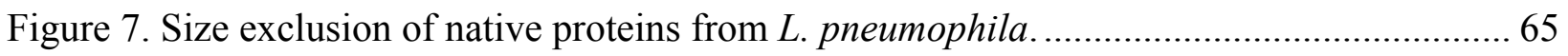

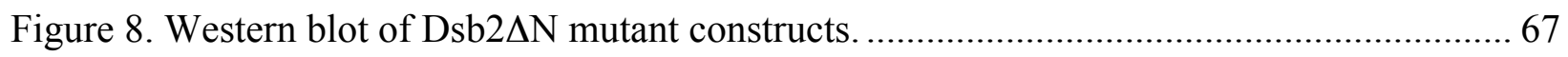

Figure 9. Complementation of motility defect in soft agar......................................................... 68

Figure 10. DsbA2 and DsbA2 $\Delta \mathrm{N}$ redox status in E.coli............................................................ 70

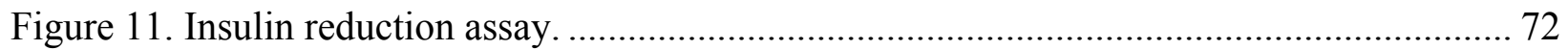

Figure 12. Complementation tests in PDI detector system..................................................... 74

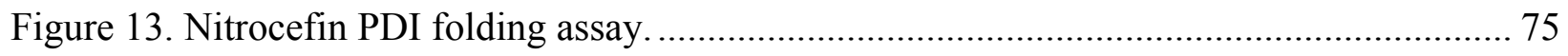

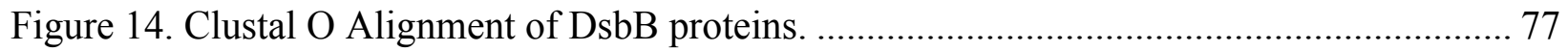

Figure 15. Clustal O Alignment of DsbD proteins. .................................................................. 79

Figure 16. Lpn DsbB1 and Lpn DsbB2 complement motility in an E. coli $d s b B$ mutant strain. . 81

Figure 17. Both Lpn DsbB1 and DsbB2 interact with monomeric DsbA2 (DsbA2 $\Delta \mathrm{N})$ in E. coli.

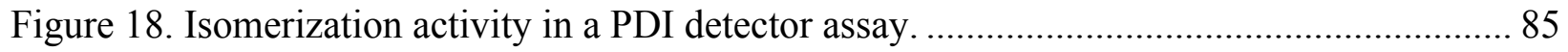

Figure 19. Lpn DsbA2 exhibits oxidase activity when both Lpn DsbB and DsbD are present.... 87

Figure 20. Redox status of Lpn DsbA2 in E. coli as determined by AMS-alkylation.................... 88

Figure 21. Disulfide bond trafficking in L. pneumophila ......................................................... 89

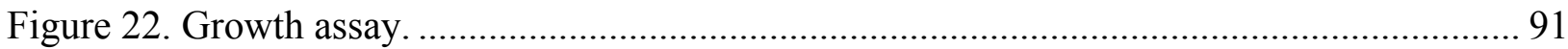

Figure 23. Intracellular replication in amoeba........................................................................ 93

Figure 24. Attachment and invasion of non-phagocytic HeLa cells by Lpn expressing wild-type and mutant DsbA2 constructs. 
Figure 25. Effects of DsbA2 wild-type and mutant constructs on Dot/Icm T4SS-mediated

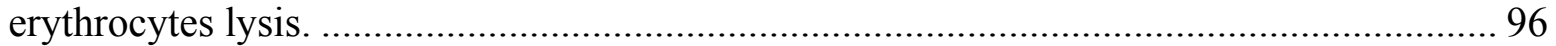

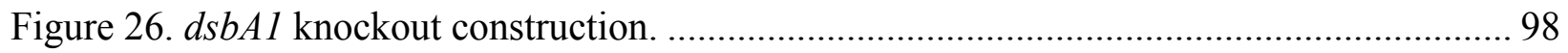

Figure 27. Protein expression and in vitro growth assessments of WT and $d s b A 1$ mutant Lpn. . 99

Figure 28. L. pneumophila DsbA1 has a defect in pigment production. 100

Figure 29. Lpn DsbA1 knockout exhibits a hypervirulent phenotype in L929 infectivity assay. 102

Figure 30. In vivo assays demonstrating the role of DsbA1 in L. pneumophila. 104

Figure 31. Substrate proteins capture by DsbA1 P150T mutant protein in L. pneumophila and E.

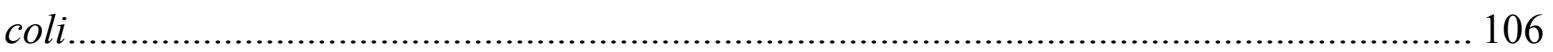

Figure 32. Crystal structures of EcDsbA and Lpn DsbA1 ................................................ 109

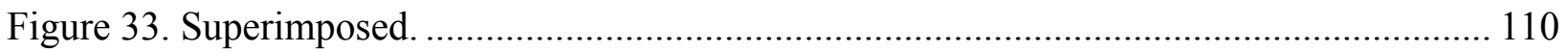

Figure 34. The thioredoxin fold and the active site of EcDsbC do not match up well with Lpn

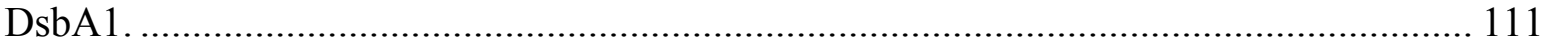

Figure 35. DsbD topological models of E. coli DsbD and Lpn DsbDs................................... 124

Figure 36. Structural model of E. coli DsbD .................................................................... 125 


\section{List of Abbreviations and Symbols}

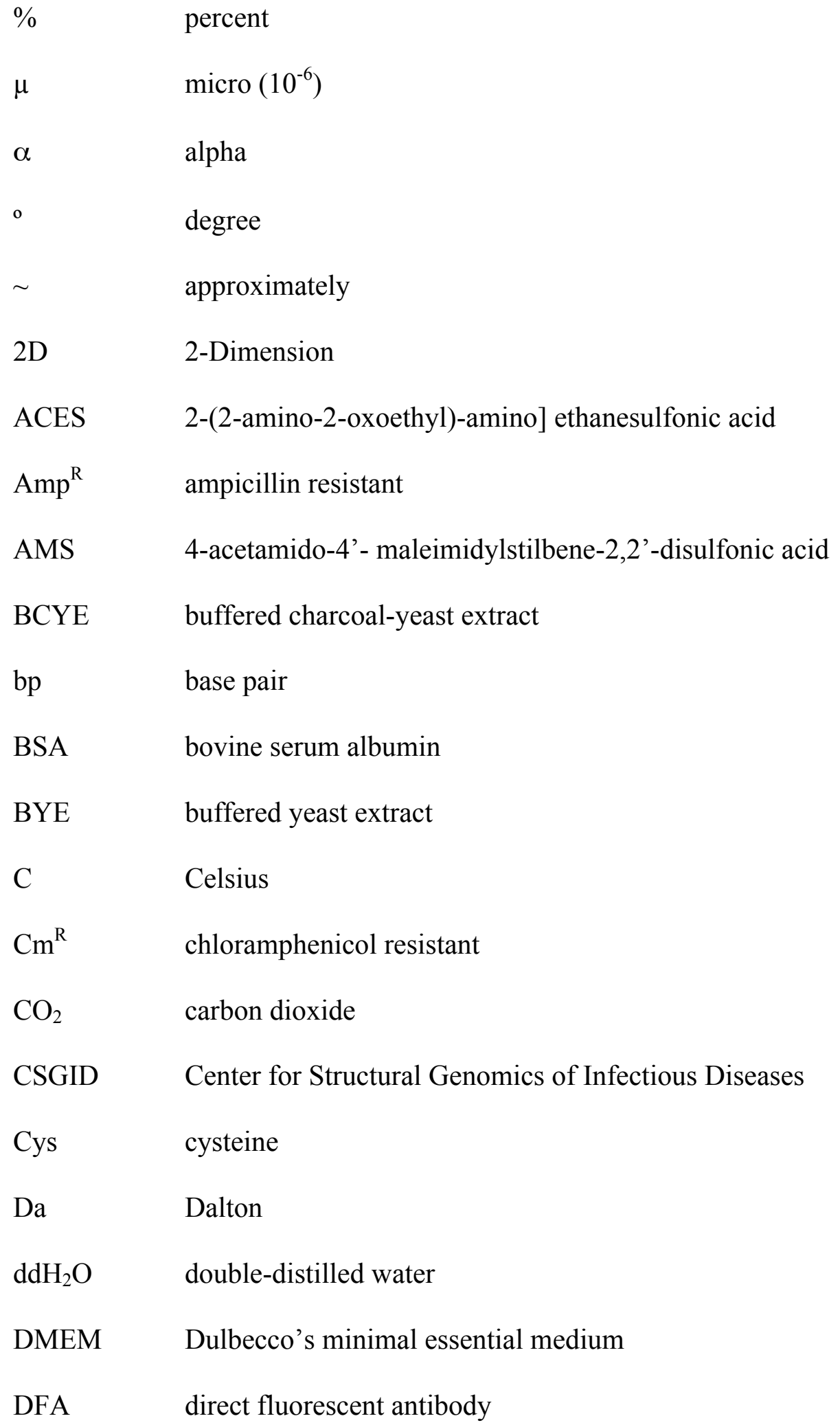




\begin{tabular}{ll} 
DMSO & dimethyl sulfoxide \\
DNA & deoxyribonucleic acid \\
Dot & defect in organelle trafficking \\
Dsb & disulfide bonds \\
DsbA1P150T & cis-proline at position 150 was changed to threonine \\
DsbA2P198T & cis-proline at position 198 was changed to threonine \\
DsbA2 $\Delta N$ & DsbA2 minus the dimerization domain sequences \\
EDTA & ethylenediaminetetraacetic acid \\
ELISAs & enzyme-linked immunosorbant assays \\
ER & endoplasmic reticulum \\
g & gram \\
$g$ & centrifugal force \\
Gent ${ }^{\mathrm{R}}$ & gentamicin resistant \\
h & hour \\
His & Legionnaires' disease \\
Icm & hexameric histidine tag \\
LPTG & intracellular multiplication \\
Km & isopropyl- $\beta$-D-galactoside \\
LCV & kanamycin resistant \\
LD & Legionella pneumophila \\
\hline
\end{tabular}




\begin{tabular}{|c|c|}
\hline LPS & lipopolysaccharide \\
\hline $\mathrm{m}$ & meter or milli $\left(10^{3}\right)$ \\
\hline M & molar \\
\hline MalPEG & $N$-ethylmaleimide covalently bound to polyethylene glycol \\
\hline $\mathrm{MH}$ & Mueller-Hinton \\
\hline MIF & mature intracellular form \\
\hline Min & minute \\
\hline MOMP & major outer membrane protein \\
\hline MW & molecular weight \\
\hline $\mathrm{N}^{++}$ & nickel \\
\hline OD & optical density \\
\hline Omps & outer membrane proteins \\
\hline PAGE & polyacrylamide gel electrophoresis \\
\hline PBS & phosphate-buffered saline \\
\hline PDI & protein disulfide isomerase \\
\hline PCR & polymerase chain reaction \\
\hline PHBA & poly $\beta$-hydroxybutyric acid \\
\hline RF & replicative form \\
\hline rpm & revolutions per minute \\
\hline ROIs & reactive oxygen intermediates \\
\hline RT & reverse transcriptase \\
\hline $\mathrm{S}$ & seconds \\
\hline S-S & disulfide \\
\hline
\end{tabular}


SD standard deviation

SDS Sodium dodecyl sulfate

$\mathrm{SH} \quad$ thiol

Strep $^{\mathrm{R}} \quad$ streptomycin resistant

T3SS type III secretion system

T4SS type IV secretion system

TAE tris acetate EDTA

TCA trichloroacetic acid

TEMED N,N,N',N'-tetramethylethylenediamine

V volt

VBNC viable but non-culturable

X-gal 5-bromo-4-chloro-3-indolyl- $\beta$-D-galactopyronidase 


\section{Acknowledgements}

First, and foremost, God is the creator of all things and when it comes to expressing the gratitude in my heart, for me that is where it begins. He gives me strength, hope, joy, peace... Phil 4:13-19, and He is unfolding the plans for my life - Jer 29:11.

In addition, there are so many people that deserve much more than a simple "thank you" for their tireless support and patience during my graduate endeavors. I would like to acknowledge those individuals whose unselfish and noble actions allowed me to be who I am today.

I would like to extend my deepest gratitude to my supervisor, Dr. Paul Hoffman for the opportunity to complete my training under his mentorship, and for his wonderful support, advice and encouragement throughout my journey as a doctoral student. I could not have imagined having any better mentor to keep me motivated with tough love as Dr. Hoffman. I also wish to express my gratitude to my supervisory committee: Dr. Alison Criss, Dr. Daniel Engel, Dr. Ian Glomski, Dr. Joanna Goldberg, Dr. Dean Kedes, Dr. Barbara Mann, and Dr. Judith White for their constructive comments, valuable suggestions, and guidance. Special thanks also go to all the members of the Department of Microbiology, Immunology \& Cancer Biology, and the Division of Infectious Diseases and International Health for their support and kindness throughout the past four years. I would especially like to thank all the members of the Hoffman Laboratory for their support, encouragement and all the good times we had to laugh and learn together; especially Dr. Shandra Day, whose professional skills and emotional support are difficult to match.

Very special thanks to my parents, Argbah Momolu and Selma Kpadeh, for your undying love and support on all things I have done and continue to do. I would thank you from the bottom of my heart, but for you my heart has no bottom.

Last, but certainly not the least, my sister Solieh Kpadeh; my many great and loyal friends and love ones for all the love, support and patience which helped me get through many obstacles in life.

Acknowledgements are also made towards the American Society for MicrobiologyRobert D. Watkins Graduate Fellowship, and NIH Biodefense training grant for their financial support of this project and my professional development. 


\begin{abstract}
Extracytoplasmic formation of disulfide bonds (DSB) is often required for proper folding of proteins, many of which are important for the assembly of virulence factors such as the Dot/Icm type IVb secretion system (T4SS) of Legionella pneumophila (Lpn), the major virulence system that delivers multiple effector proteins into the cytoplasm of host cells. In Escherichia coli, DsbA catalyzes the formation of disulfide bonds, while DsbC repairs inappropriate disulfides through protein disulfide isomerase (PDI) activity. DsbA is a monomer that is maintained in a fully oxidized state by the cytoplasmic membrane protein DsbB, whereas DsbC is a homodimer and kept reduced by a second cytoplasmic membrane protein, DsbD.

In contrast, Lpn expresses two DsbA-like proteins (DsbA1 and DsbA2), but no orthologue of DsbC. Both DsbA proteins are capable of catalyzing DSB formation; however, $d s b A 1$ is dispensable for viability and virulence, while $d s b A 2$ is essential and required for disulfide bond formation in core proteins of the Dot/Icm type IVb secretion system. DsbA2 is a homodimer that is phylogenetically distinct from DsbA and DsbC lineages. We hypothesized that in a $d s b A 1$ mutant of Lpn, DsbA2 must be responsible for both oxidase and PDI activities; i.e., a single player system as compared to the two player DsbA/DsbC system of E. coli.

In this study we confirmed that DsbA2 possesses PDI activity using a PDI detector assay in which DsbA2 replaces DsbC in E. coli. Consistent with a single player bifunctional system, we showed that DsbA2 exists in the periplasm of Lpn as a mixture of disulfides (S-S) and free thiols (SH). This equilibrium can only occur if the two DsbB oxidases (DsbB1 and DsbB2) cooperate with the two DsbD reductases (DsbD1 and DsbD2). To test this hypothesis, we reconstituted the Lpn Dsb system in various $d s b$ mutants of $E$. coli, including a $d s b$ null mutant strain. Our studies showed that DsbA2 did not restore motility to a $d s b A$ mutant of E. coli, whereas a monomeric dimerization domain mutant $(\mathrm{DsbA} 2 \Delta \mathrm{N})$ restored motility. We showed that both DsbBs of Lpn were able to oxidize DsbA2 $\Delta \mathrm{N}$, but not DsbA2 and that the E. coli DsbD reduced DsbA2 to the thiol. Motility was restored to a $d s b$ null mutant of $E$. coli expressing Lpn $d s b A 2, d s b B 1$ or $d s b B 2$ and $d s b D 1$ or $d s b D 2$ and this was possible because the reconstituted system maintained DsbA2 as a mixture of S-S/SH forms. This specificity of Lpn DsbB and DsbD proteins for DsbA2 bifunctional activity suggests a distinct evolutionary tract for the DsbA2 system.
\end{abstract}


In addition, we identified biological roles for nonessential DsbA1 in Lpn, by showing that a $d s b A 1$ mutant had lost the ability to produce melanin-like pigment and was more infectious of HeLa cells. This mutant was also more virulent by the contact hemolysis assay, and all of these phenotypes were restored to WT through complementation. Finally, overexpression of DsbA1P150T mutant protein in Lpn did not result in a dominant negative effect on DsbA2 function as was seen with either the DsbA2P198T or DsbA2 $\Delta$ NP198T mutant proteins. Taken together, these studies suggest that DsbA1 might compete for substrate proteins with DsbA2 and perhaps modulate virulence.

These findings indicate that DsbA2 is a bifunctional oxidoreductase in Lpn, which is maintained by the DsbB and DsbD proteins that cooperate in maintaining this single player system. In addition, this protein, which is found in many other pathogens expressing type IV secretion systems, likely provides a selective advantage by economizing disulfide bond management in these slow growing bacteria. 


\section{Chapter 1. Introduction}

\subsection{Legionella pneumophila Review}

An outbreak of severe respiratory illness, now known as Legionnaires' disease, occurred in the summer of 1976 in Philadelphia, Pennsylvania. On July 30, 1976, Dr. Joseph Campbell realized that he was treating several patients for an atypical pneumonia, chiefly among veterans who attended the American Legion convention (Fraser et al., 1977). All of the infected individuals had either stayed as guests at the Bellevue-Stratford hotel, had spent time in the lobby or on the sidewalk immediately outside the building. After returning to their respective hometowns from the convention, over 182 people came down with this unknown illness, 29 of which died. Of the 29 who succumbed to Legionnaires' disease most were males over 55 years of age, who were immunocompromised, or with respiratory problems (smokers), and or other underlying comorbidities. Interestingly, the pneumonic illness was not spread to immediate family members or to healthcare workers in close contact with the infected victims. An extensive laboratory investigation was undertaken by the Pennsylvania State Health Department and the National Centers for Disease Control, where techniques for detecting a wide variety of toxins and for identifying infections caused by chlamydia, fungi, mycoplasmas, parasites, rickettsia and viruses were employed. Despite these efforts, the etiologic agent of the disease was not identified.

Six months after the initial report of the epidemic, Dr. Joseph McDade from the Centers for Disease Control, first noted that histological slides of human lung biopsy material and spleen tissue from experimentally infected guinea pigs contained a common bacterial "contaminant" (McDade et al., 1977). Convalescent serum confirmed the Gram-negative bacterium as the etiological agent of the disease, and was subsequently named Legionella pneumophila honoring 
the victims who brought national attention to the disease. Since the bacterium had not been isolated on routine bacteriological media, special medium was developed for its isolation and cultivation (Feeley et al., 1979). These developments allowed epidemiologists to eventually identify the air-conditioning system of the Bellevue-Stratford hotel as responsible for the dissemination of the organisms to the veterans (Blackman et al., 1978). Interestingly, the sera of the hotel employees, who had experienced none of the symptoms of the disease, tested positive for previous exposure to the organism. This indicated that the bacterium was an opportunistic pathogen, causing illness primarily in physically debilitated or immunocompromised individuals. Retrospective studies revealed that other epidemics of Legionnaires' Disease (LD) had occurred prior to 1976, and that the disease Pontiac Fever was caused by Legionella pneumophila (Kaufmann et al., 1981). Scientific research over the last 40 years has yielded vast amount knowledge into the etiology and pathogenesis of this disease; yet, detailed information about the role of disulfide bonds proteins in the periplasm for the folding of virulence factors for the pathogenesis of Legionella still remains a mystery.

\subsection{Clinical relevance}

Legionella pneumophila is also responsible for causing a less severe disease - a mild flu-like illness called Pontiac Fever (Glick et al., 1978), and Legionnaires' disease (LD), a more severe multi-system infection that causes severe pneumonia (Fraser et al., 1977), and despite antibiotic intervention it is often fatal. L. pneumophila is the predominant cause of legionellosis in humans, accounting for $\sim 80-90 \%$ of all reported cases in the United States with a predominance for serogroup 1 (Fields et al., 2002). 
Pontiac fever was first documented from a flu-like outbreak that struck workers in 1968 in Pontiac, Michigan, but L. pneumophila was only identified as the etiological agent ten years after the incident (Glick et al., 1978). Infected individuals displayed mild symptoms such as malaise, myalgia, and headaches, and usually recovered without hospitalization within five days (Glick et al., 1978). In contrast, LD presents as an atypical pneumonia with diffuse radiographic infiltrates within 2 to 10 days after initial exposure to Legionella, and with more severe symptoms that may include fever, muscle aches, chest pain, dry cough, abdominal pain, diarrhea, neurological problems and urinary retention (McDade et al., 1977; Fraser et al., 1977). The annual incidence of LD in the United States is estimated at $>100,000$ cases (Fields, 1996; Bitar et al., 2004). This number may underestimate the true number of cases, as up to $50 \%$ of pneumonia is of unknown etiology. Outbreaks of disease can be traced to cooling towers, hot water systems, potable water, humidifiers, hot tubs, and many more other sources (Wadowsky et al., 1982). LD is always transmitted from the environment to people via L. pneumophila-laden aerosols and there are no reported cases of human-human transmission. L. pneumophila remains a significant threat to the public because it continues to cause random epidemics that often involve hundreds of people over distances up to 14 kilometers from the point source (Ngyen et al., 2006). The mortality rate can exceed $25 \%$ in debilitated individuals (cancer patients, elderly, immunocompromised persons and solid organ transplant patients) (Wadowsky et al., 1982; Winn, 1988; Marrie, 1993; Fields et al., 2002). In addition to the morbidity and mortality it causes, Legionella is studied because its intracellular life cycle is similar to other existing and emerging human pathogens, and for its weapon potential in studying transmission by aerosol. 


\subsection{Legionella Family and Taxonomy}

To date, more than 50 species of Legionella have been identified, and at least 24 of these have been associated with human disease (Newton et al., 2010). Lpn is the predominant cause of legionellosis in humans, accounting for $\sim 90 \%$ of cases, with serogroup 1 accounting for $84 \%$ of $L$. pneumophila pneumonia (Yu et al., 2002). Some species of Legionella can be further differentiated into serotypes, of which there are at least 15 for L. pneumophila but so far no more than two for any other species. Within the type species Lpn, 15 serogroups have been identified (Benson \& Fields, 1998). The variability between the serogroups has largerly been attributed to the diversity in antigens (lipopolysaccharide, LPS) expressed on the surface of the bacterium. Since Lpn accounts for 90\% of human cases of disease (Marston et al., 1994), this Legionella species has received the most attention. While Legionella serogroup 1 is found in the environment, it is not the dominant species worldwide. In France, studies have found that serogroup 1 comprises $28 \%$ of environmental isolates but accounts for $94 \%$ of clinical isolates (Doleans et al., 2004). Of the remaining cases, the majority are caused predominantly by $L$. longbeachae (4\%) and L. bozemanii (2.4) (Muder and Yu, 2002). Based on a single reported human infection, some of these species are classified as pathogenic (Newton et al., 2010).

Lpn, a Gram-negative coccobacillus belongs to the gamma-subgroup of proteobacteria. Comparison of the DNA relatedness of the Legionnaires' disease bacterium, Lpn to known bacterial isolates led researchers to propose that Legionella be placed in a new family, the Legionellaceae (Brenner et al., 1979). Later 16S rRNA analysis demonstrated that Rickettsia and Coxiella species show distinct evolutionary relatedness with Legionella. In 2004, the completed Legionella genome sequence revealed that Lpn shares distinct evolutionary relatedness by sharing $\sim 42 \%$ of its genome (3.4Mbp) with the Coxiella burnetii genome (1.9Mbp), an intracellular 
pathogen causing Q fever (Chien et al, 2004). Several known and putative virulence factors are among the shared genes (Seshadri et al., 2003; Seshadri and Samuel, 2005). The novel DsbA2 protein of Lpn, which is futher characterized in this study was originally annotated as Com1, an orthologue of the Com1 protein of Coxiella (Jameson-Lee et al., 2011). Coxiella is genetically related to Lpn, including genes encoding the highly conserved Dot/Icm T4SS, and orthrologues of $d s b B, d s b D$, and DsbA2. Since Coxiella does not encode DsbA1, by default, DsbA2 must be bifunctional in this bacterium.

\subsubsection{Microbiology of Legionella pneumophila}

Legionella pneumophila is an aerobic, non-sporulating Gram negative intracellular pathogen that is 0.3 to $0.9 \mu \mathrm{m}$ in width and 2 to more than $20 \mu \mathrm{m}$ in length (Brenner et al., 1979; Brenner, 1986); it is responsible for most cases of Legionnaires' disease worldwide (Fraser et al, 1977; Horwitz, 1983 a and b). The bacteria possess flagella and are thus considered motile. Except for L. oakridgenesis, L. brunesis, L. cincinnatiensis, and L. longbeachae serogroup 1, the other named species are motile by one, or two polar or lateral flagella (Chandler et al., 1980; Rodgers et al., 1980; Bornstein et al., 1991).

In natural environments, L. pneumophila multiplies in protozoa and acquires traits that increase its virulence (Cirillo et al., 1994; Fields et al., 2002; Greub and Raoult, 2004). In fact, $L$ pneumophila displays a developmental cycle in protozoa and some mammalian cell lines, where the organisms differentiate into metabolically dormant cyst-like forms that enable the bacteria to survive for extended periods in a highly infectious state (Garduno et al., 2002; Greub and Raoult, 2004). Transmission to susceptible humans occurs via inhalation of Legionella-contaminated aerosols where L. pneumophila infects alveolar macrophages (Fields et al., 2002). By virtue of 
virulence factors such as the Dot/Icm type IVB secretion system, L. pneumophila avoids lysosomal degradation by generation of a replication-permissive endosome that fails to mature via the endocytic pathway (Berger and Isberg, 1993; Horwitz, 1983 a and b; Marra et al., 1992). Evasion of phagolysosomal fusion is a particularly apt strategy to avoid being consumed by protozoa or killed by human alveolar macrophages and as such will be discussed in a future section (1.3.3).

In terms of their laboratory growth requirements, the organisms are fastidious. They do not grow on blood agar or other standard laboratory media. Their source of carbon and energy is derived from amino acids (George et al., 1980). The bacteria rely on cysteine (cys) and iron, and an optimal $\mathrm{pH}$ of between 6.0 and 6.9 for growth (Feeley et al., 1979; Edelstein, 1981) The growth medium that is currently used is buffered charcoal yeast extract (BCYE) agar supplemented with cys and $\alpha$-ketoglutarate, and has a pH of 6.9 (Feeley et al., 1979, Edelstein, 1981). The charcoal in the media tends to neutralize toxic compounds such as hydrogen peroxide and superoxide radicals that are found upon exposure of the media to fluorescent light, and whose levels are accelerated upon autoclaving (Hoffman et al., 1983). The organisms are generally grown at 35 to $37^{\circ} \mathrm{C}$ in a $\mathrm{CO}_{2}$ incubator. Legionella colonies, which are bluish-grey in color, are usually visible on BCYE plate after three to four days after inoculation.

\subsubsection{Diagnosis}

Post-mortem human lung tissue was used to infect guinea pigs and spleen homogenates were inoculated into embryonated yolk sacs, resulting in the original discovery of the etiological agent of Legionnaires' disease (McDade et al., 1977; Brenner et al., 1979). The resulting bacteria were visualized with Gimenez stain, which uses carbol fuchsin to intensely stain Gram-negative 
bacteria and Rickettsia. The use of the Gimenez stain was critical, as traditional staining methods failed to detect any bacteria despite the tremendous numbers of bacteria present in the lung in fatal cases (Greer et al., 1980). In addition, early attempts to culture Legionella on common laboratory media such as Mueller-Hinton (MH) agar were unsuccessful (Feeley et al., 1979). A better diagnostic technique was established when a growth medium supporting Lpn growth was discovered. Addition of hemoglobin and IsoVitaleX permitted growth, resulting in the identification of soluble iron and L-cysteine as essential components of the Feeley-Gorman agar (Feeley et al., 1979).

Since then, routine laboratory culture of virulent Lpn was made possible using charcoal yeast extract agar in which starch was replaced with charcoal and essential amino acids were provided in yeast extract (Feeley et al., 1979). Iron salts like ferric pyrophosphate establish equilibrium between cysteine and the oxide dipeptide cystine, maintaining a steady-state level of cysteine that promotes growth (Ewann and Hoffman, 2006). The $\alpha$-ketoglutaric acid also serves as a primary carbon source for Legionella (Tesh and Miller, 1981). Further refinements like addition of ACES buffer and charcoal led to the currently used buffered charcoal-yeast extract (BCYE) medium (Pascule et al., 1980). Charcoal, $\alpha$-ketoglutaric acid, and cysteine were shown to scavenge reactive oxygen intermediates (ROIs) generated in the medium during aerobic growth (Hoffman et al., 1983; Pine et al., 1986).

Legionella can be defined in culture by its requirement for L-cysteine, serologically by direct fluorescent antibody (DFA), enzyme-linked immunosorbant assays (ELISAs), polymerase chain reaction (PCR), real-time PCR, and by DNA sequencing (Fields et al., 2002). 


\subsubsection{Microbial Ecology}

Paradoxically, the natural habitat of Legionella is aquatic, where the nutrient levels and the general growth conditions are expected to be poor. They have been isolated from both natural aquatic environments, i.e. rivers, lakes, streams, and thermally polluted waters (Nguyen et al., 1991) as well as manmade aquatic environments, i.e. cooling towers, hot-water systems, whirlpool spa baths, clinical humidifiers in respiratory equipment, and a supermarket vegetable sprayer (Lee and West, 1991). Also, in an outbreak of Legionnaires' disease in Australia where $L$. longbeachae was identified to be the causative agent, this organism was isolated from potting soil compost used by the patients, who were all avid gardeners (Steel et al., 1990). In the aquatic environment, Legionella have been isolated from water temperatures ranging from $5.7^{\circ} \mathrm{C}$ to $63^{\circ} \mathrm{C}$ (Fliermans et al., 1981).

Legionella are able to survive for extended periods of time in aquatic biofilms (Rogers et al., 1994) where they fall prey to grazing amoeba (Greub and Raoult, 2004). Bacterial-protozoa interactions not only represent a shelter against stress, but also serve as a niche for replication and selection of virulence traits that prime the pathogen for human infection (Greub and Raoult, 2004). Excluding environmental protozoa, biofilms can provide nutrients and shelter from environmental stresses and thus could provide a suitable environment for persistence (Fields et al., 2002). Lpn can survive for extended periods of time as a viable but non-culturable (VBNC) form; however, it is less clear whether the bacterial is able to replicate in biofilms without protozoa (Steinert et al., 1997). 


\subsubsection{The Developmental cycle of Legionella}

As a means of ensuring survival, many intracellular organisms have adopted a variety of mechanisms to allow persistence within their adapted niches (Finlay \& Falkow 1997). Intracellular parasitism is not unique to Legionella. Despite what is most likely to be an inhospitable environment, several bacteria including Salmonella, Shigella, Yersinia, Mycobacteria, Chlamydia, Coxiella and Listeria all prefer, or seem to have adapted to intracellular lifestyles (Moulder et al., 1984; Finlay \& Falkow, 1997). Selective pressures distinct to each niche favor certain traits that maximize survival of the organism in its habitat. One mechanism to allow survival in aquatic environments is transition to a cyst-like form when environmental conditions are poor; when the proper nutrients become available, the organism then differentiates into a metabolically active vegetative form (Sadoff, 1976). A number of Gram-negative intracellular pathogens have evolved a similar differentiation mechanism to allow their persistence, though the mechanism is modified slightly for each of their individual intracellular lifestyles. Like Legionella and Chlamydia, C. burnetii possesses a developmental cycle that was recognized as early as the 1950's (Heinzen, 1997) when density gradient centrifugation yielded a variety of bacterial forms with different buoyant densities. Coxiella alternates between a replicative large-cell variant and the environmentally resistant small-cell variant (Samuel et al., 2003). Similarly, the differentiation of Lpn in protozoa and mammalian cells has been well documented (Figure 1). Byrne and Swanson (1998) provided a detailed investigation of the changes associated with Lpn growth in vitro. During exponential phase, Lpn 


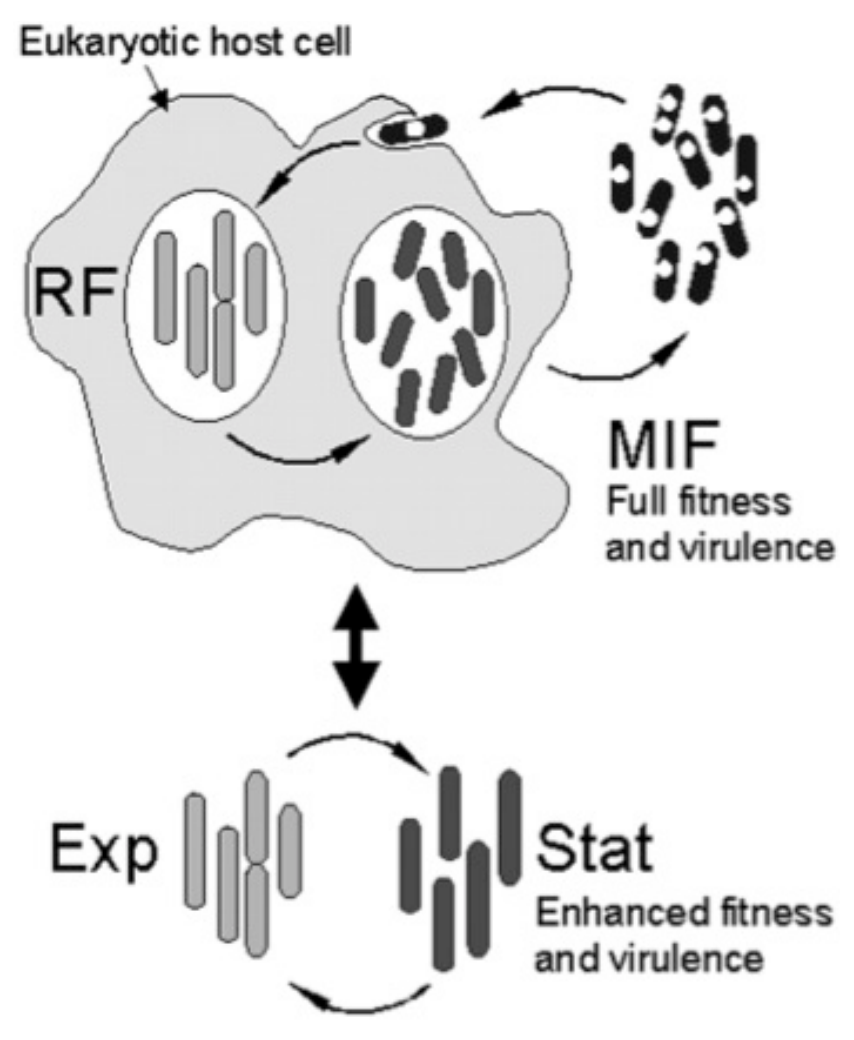

Figure 1. Simplified model illustrating the intracellular and extracellular growth cycles of L. pneumophila. Only the intracellular growth cycle produces the fully differentiated mature intracellular form (MIF), which is more infectious and environmentally fit than the in vitro-grown SP bacteria (Stat). Presumably, host-derived signals (inducers) are required for post-stationaryphase differentiation of MIFs. Intracellular replicative forms (RFs) would be equivalent to exponentially growing bacteria (Exp) in vitro, as they display similar morphological traits, but it remains to be determined whether or not they are physiologically different. The central double arrow indicates that Lpn can move from one cycle to the other when the proper conditions are met. Notice that, to initiate a new intracellular cycle, Lpn has to exit into the aquatic environment, where it is possible for MIFs to continue their maturation in response to extracellular signals (inducers). Adapted from Garduño et al., 2002. 
was most resistant to salt (a phenotype often associated with avirulent Lpn strains) (Catrenich \& Johnson, 1989), less cytotoxic, less osmotically tolerant, less motile, and less infectious. Based on in vitro growth studies, virulence was shown to increase post-exponentially. Similar changes were observed when Lpn were intracellular; intracellular forms were salt-tolerant and non-motile, but upon host cell lysis, the bacteria were observed to be flagellated and sodium sensitive.

As depicted in Fig. 1; throughout the infection of host cells, Lpn is shown to alternate between a replicative form (RF) and a metabolically dormant, highly infectious cyst-like form termed the mature intracellular form (MIF) (Garduño et al., 2002). The stage-specific transition is emphasized by the various intermediate (RF-to-MIF or MIF-to-RF) morphological forms (Faulkner and Garduño, 1998). RFs and MIFs could be distinguished by Giménez staining (Fig. 2), where MIFs retained the carbol fuschin red color (Giménez-positive) whereas RFs, being unable to retain the stain, stained green by the malachite green (Giménez-negative) counterstain (Garduño et al., 2002). Compared to RFs, MIFs were metabolically dormant and displayed numerous ultrastructural differences, including poly $\beta$-hydroxybutyric acid (PHBA) inclusions, a thickened cell-wall architecture, and multi-laminated intra-cytoplasmic membranes, which were all reflected by increased resistance to various stress (Garduño et al., 2002). It should also be noted that due to early demise of the host, Lpn does not differentiate into MIF-like forms very efficiently in infected macrophages, which may partly explain why LD is not a communicable disease (Garduño et al., 2002). Nonetheless, Lpn progeny arising from intracellular growth in macrophages express numerous transmission traits that could facilitate their uptake by other phagocytes (Molofsky and Swanson, 2004). It is also important to note that in vitro, MIFs do not form because development is arrested in stationary phase. For intracellular pathogens that have a 


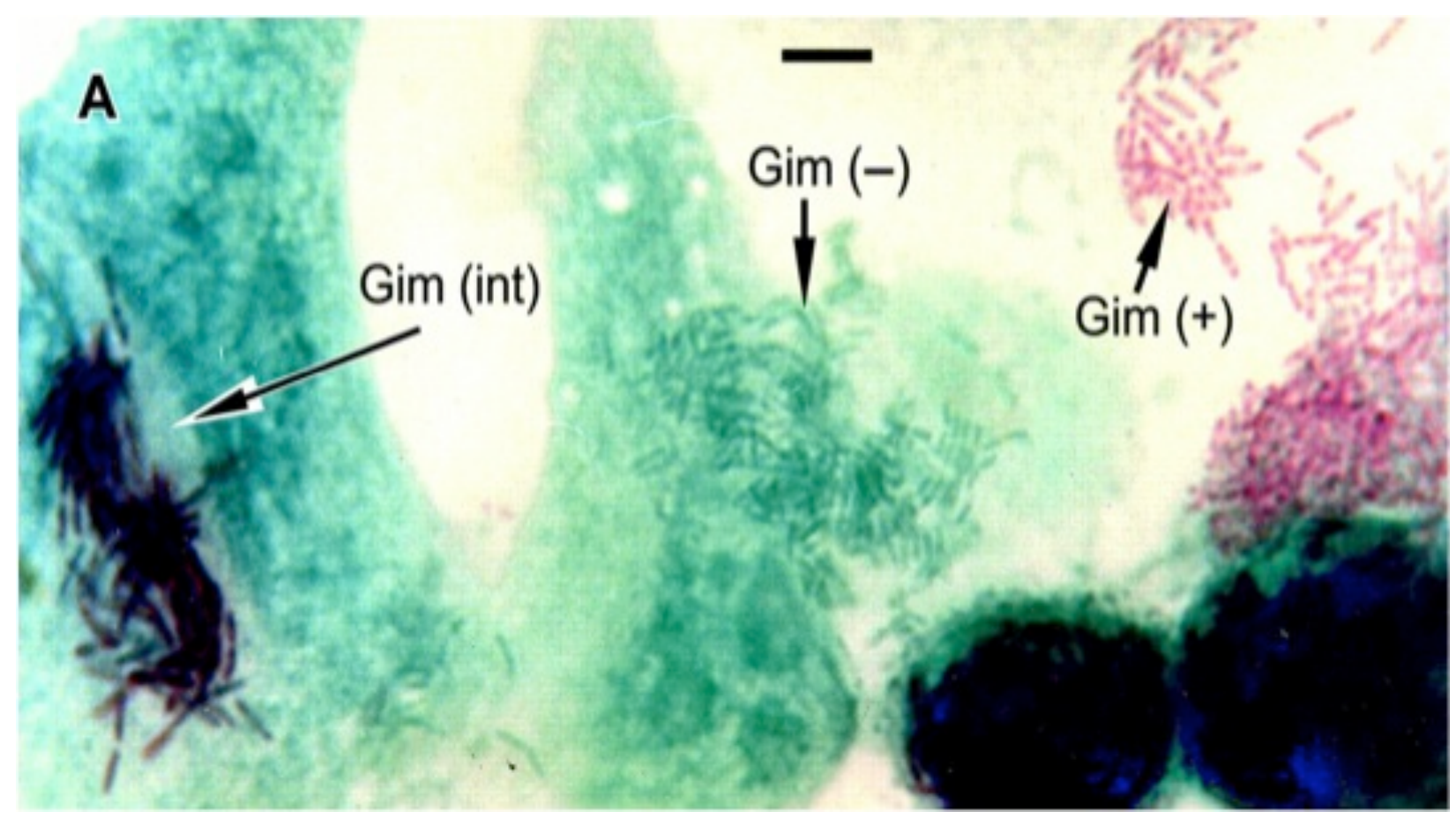

Figure 2. Morphological differences between the two principal forms of L. pneumophila in vivo. Giménez phenotypes observed in HeLa cell monolayers infected with purified MIFs. Light micrograph showing three different Giménez phenotypes $(+,-$, and int) in a single microscopical field from a specimen fixed and stained $24 \mathrm{~h}$ after infection. The Giménez negative (green, -) replicative forms are believed to be the form of Lpn that replicates within the host cell. The intermediate (int) forms are believed to represent a transition phase between the replicative forms and the mature intracellular forms (MIFs), which stain red with the Giménez stain, but lack the discontinuous staining pattern of the MIFs $(+)$ formed during later stages of infection. Scale bar $=10 \mu \mathrm{m}$. Adapted from Garduño et al., 2002. 
developmental cycle, there is probably no well-defined stage for stationary phase - they might just go right to differentiation.

\subsection{Virulence of Legionella in protozoa and Human hosts}

The resistance of Legionella to these harsh environmental conditions may be explained by the observation that these bacteria can maintain a commensal association with other aquatic microorganisms. The first evidence for symbiosis between Legionella species and other organisms was presented by (Tison et al., 1980), where Lpn was isolated from an algal mat in thermally polluted water. In the same year, Rowbotham (1980) reported that Legionella can infect amoebal trophozoites and then become incorporated in their cysts. To date, Lpn has been found to replicate in numerous amoeba, ciliates, and slime mold (Abu Kwaik et al., 1998; Fields et al., 2002; Steinert et al., 2002). Acanthameoba castellanii has been shown to resuscitate Lpn from its environmental VBNC state, revealing important ecological significance (Steinert et al., 1997). Growth with protozoa protects Lpn from chlorination, which may explain why elimination of Legionella from water systems is so difficult. Indeed, continuous treatment with chlorine dioxide was identified as the most efficient method for controlling Lpn in water systems; however, presence of protozoa resulted in quick re-colonization by Lpn (Thomas et al., 2004). In 1986, Rowbotham suggested that Legionella-infected amoeba might represent an important vehicle for human transmission. This hypothesis was further confirmed in a murine model where co-infection of Lpn and protozoa resulted in a more severe pneumonia than with either organism alone (Brieland et al., 1996). Rowbotham (1986) also suggested that Legionella-filled vacuoles could represent a transmissible form. As further demonstrated in A. castellanii or polyphaga, Lpn was expelled in vesicles that could easily be inhaled ("respirable"), and deliver a substantial dose of 
organisms (Berk et al., 1998). Latter studies showed that the release of vesicles from protozoa was attributed to two proteins, LepA and LepB (Chen et al., 2004) that were translocated into the host cells by the Dot/Icm system (to be described in detail in section 1.5) (Fig. 3). It is important to note that intracellular growth in environmental protozoa is known to select for traits promoting environmental survival (Greub \& Raoult, 2004; Rowbotham, 1986) such as resistance to biocides, cold, antibiotics, or traits required for the intracellular lifestyle within human macrophages (Brüggemann et al., 2006; Greub \& Raoult, 2004)

Legionella's ability to replicate within many different protozoa has equipped the bacteria with the capacity to replicate in human alveolar macrophages (Abu Kwaik, 1996). Legionella growth in environmental hosts has been studied with Acanthamoeba castellani, Hartmanella vermiformis, Naegleria spp., and Dictostelium discoideum (Valster, et al., 2010). After replicating in these hosts, disseminated Lpn invades and replicates within human alveolar macrophages, monocytes, and alveolar epithelial cells (Gao et al., 1998). After phagocytosis, the facultative intracellular pathogen Lpn is found within a membrane-bound compartment, termed the Legionella-containing vacuole (LCV), which diverges from the endosomal-lysosomal pathway within five minutes (Horwitz, 1983b; Roy et al., 1998). The LCV fuses transiently with mitochondria, subsequently becoming surrounded by ribosomes and does not fuse with primary or secondary lysosomes (Horwitz, 1983b). Formation of the LCV involves recruiting secretory vesicles from the endoplasmic reticulum (ER) (Kagan and Roy, 2002), which then proceed to remodel the LCV to resemble an ER-like vacuole (Horwitz, 1983; Swanson and Isberg, 1995; Tilney et al., 2001). Egress from spent hosts is thought to occur via cell death (Gao and Abu Kwaik, 1999) caused by pore formation in the host plasma membrane (Alli et al., 2000; Kirby et al., 1998). In addition to possessing traditional bacterial virulence determinants such as 


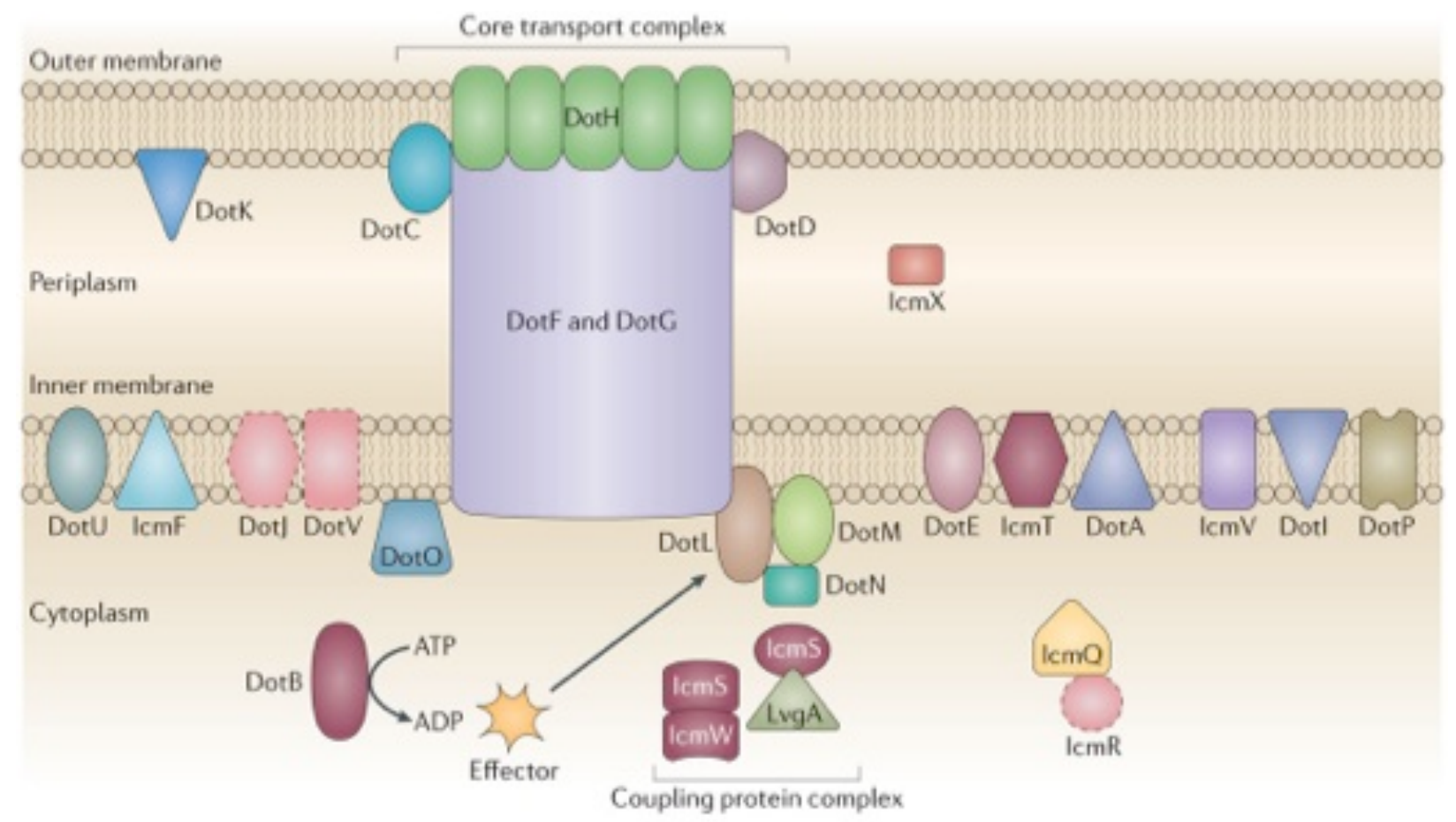

Figure 3. The type IVB secretion systems of Legionella pneumophila. The proposed structure of Lpn T4bSS based on mutational analysis encoding dot (defect in organelle trafficking) and icm (intracellular multiplication) proteins. Studies using Lpn identified two subcomplexes: first termed the core transport complex, which links the inner and outer membranes and is composed of DotC, DotD, DotF, DotG and DotH. A second subcomplex consists of the coupling protein DotL (providing a link between the substrates and the transport complex), DotM, DotN, IcmS and IcmW. Adapted from van Schaik et al., 2013. 
lipopolysaccharide (LPS) and flagella, Legionella has a unique Dot/Icm type IV secretion system (T4SS) (Fig. 3), which serves as a conduit that injects over 300 effector proteins into the host to manipulate host cell processes.

\subsection{Dot/Icm Type IV Secretion System (T4SS)}

Genetic analysis has revealed Lpn contains two T4SSs: T4aSS, which is more similar to the Agrobacterium tumafaciens Vir system, and the T4bSS, first studied in Legionella that is more similar to Incl plasmids (Newton et al., 2010). Of these two systems, only Dot/Icm T4bSS is known to play a crucial role in virulence (Marra et al., 1992; Berger \& Isberg, 1993; Segal et al., 1999). Despite their diversity, all T4SS are thought to be evolutionarily related and probably functionally similar (Lessi \& Lanka, 1994). For the interest of this report, the Dot/Icm T4bSS is discussed below.

The Dot/Icm T4SS (Fig. 3) of Lpn was discovered simultaneously by two independent groups (Marra et al., 1992; Berger \& Isberg, 1993), and is now known to be encoded by 26 genes of various nomenclatures found on two distinct regions of the Legionella chromosome designated dot for "defect in organelle trafficking" and $\mathrm{icm}$ for "intracellular multiplication" (Vogel et al., 1998, Segal et al., 1998, Sexton \& Vogel, 2002). Phagosomes containing Legionella dot/icm mutants fail to evade the endocytic pathway and quickly acquire endosome markers producing an avirulent phenotype. Though the global architecture of the Dot/Icm system is yet to be elucidated, the macromolecular complex is thought to act as a molecular syringe to inject effector proteins that modulate host cell functions into the host cell upon contact activation within minutes of bacterial internalization (Roy et al., 1998; Wiater et al., 1998; Coers et al., 1999). The T4SSs are important for the virulence of several pathogens, including Agrobacterium tumafaciens, 
Helicobacter pylori, Bordetella pertussis, Bartonella sp., Brucella sp., Ricketssia sp., Coxiella burnetii, and Wolbachia sp. (Backert \& Meyer, 2006). Lpn utilizes the Dot/Icm T4SS to inject effector proteins into host cells, most of which have unknown effects or may be reductant, rendering them unsuitable to mutational analysis (Hubber \& Roy, 2010). Functions for some Dot/Icm effector proteins have been reported; sdeA regulates uptake of Lpn into host cells (Bardill et al., 2005), AnkX allows Lpn to evade the host endocytic maturation pathway (Pan et al., 2008), LegC3 (de Felipe et al., 2008), and Dot F (Paumet et al., 2009), recruit ER-derived vesicles to the LCV (DrrA (Machner \& Isberg, 2006), LepB (Ingmundson et al., 2007), SidD (Tan \& Luo, 2011)), and inhibit host cell apoptosis (SdhA and SidF (Banga et al., 2007; Laguna et al., 2006)). The formation of correct DSB in the proteins required for the assembly of the Dot/Icm T4SS apparatus is essential for proper folding and function (to be discussed in detail in section 1.6). While the core proteins require for the assembly of the Dot/Icm T4SS apparatus have been described (Vincent et al., 2006), the roles of the disulfide bonding proteins involved in the proper folding of these core proteins in Legionella have not been elucidated.

\subsection{Protein Folding and Disulfide Bond Formation}

In Gram-negative bacteria, membrane or secreted proteins are first expoerted to the periplasm. They are initially synthesized in the cytoplasm, and then translocated across the inner membrane by different export mechanisms (Wandersman, 1992). As newly synthesized polypeptides emerge into the periplasmic space, they must fold into their three dimensional configuration in order to achieve their native structure and be functional later in their final location (Missiakas \& Raina, 1997a and b). For proteins that contain intramolecular disulfide bonds, such as the core proteins (DotC ( 2 cys), DotG (15 cys), DotK ( 3 cys) and IcmX ( 3 cys)) of the Dot/Icm T4SS apparatus, 
proper folding is dependent on correct formation of new bonds or isomerization of existing disulfide bonds. These bonds are often essential for the stability of the tertiary and quaternary protein structures and their function (Bardwell \& Beckwith, 1993; Luckey et al., 1991; Gething \& Sambrook, 1992). Reduction or breakage of disulfide bridges leads to unstable or unfolded proteins and inactive catalytic enzymes (Bardwell et al., 1991; Bardwell et al., 1993; Bardwell \& Beckwith, 1993; Dailey \& Berg, 1993; Missiakas et al., 1993b). These bonds are formed by the linkage of pairs of cysteines in unfolded polypeptides and involve the transfer of a disulfide bond from an external donor that then becomes reduced. The disulfide bond donor in Gram-negative bacteria is an oxidase, which is located in the bacterial periplasm. A disturbing factor in the kinetics of renaturation of reduced proteins was that it took them hours to fold in vitro, but only minutes in a cell (Anfinsen et al., 1961; Canfield \& Anfinsen et al., 1963). This discrepancy led to the search for and discovery of an enzyme eventually identified as protein disulfide isomerase (PDI), which catalyzes the proper formation of DSBs (Venetianer \& Straub, 1963; Canfield \& Anfinsen et al., 1963) in proteins. It is exciting to note that Bruno Straub later became the President of Hungary, and Christian Anfinsen won a Nobel Prize for his work on protein folding.

As previously stated above, protein folding has been studied for many years, and has been shown to occur spontaneously and slowly in vitro (Anfinsen, 1973). Specifically, in vivo, protein folding occurs so rapidly that it must be assisted by accessory proteins, especially in response to environmental conditions such as changes in temperature, $\mathrm{pH}$ or osmolarity (Gross, 1996). The role of these accessory proteins is not only to assist folding into native structure, but also to prevent degradation of unfolded proteins. Although PDI had been discovered many years previously, no one had looked for an enzyme similar to PDI in bacteria; it was assumed that the oxidizing environment of the periplasm was enough to cause DSB to form spontaneously. By the 
1990's, two labs discovered $d s b$ A (DsbA) in E. coli and Vibrio cholerae (Bardwell et al., 1991; Peek \& Taylor, 1992), leading to the characterization of these processes in bacteria.

DSB is the covalent link formed between the thiol groups of two cysteine residues when they are in an oxidizing environment, and when the redox potential of the cysteine residues is below that of the biological system it is in. The redox potential of any species is a measure of its affinity for electrons compared against a standard hydrogen electrode with a half-cell potential of $0.0 \mathrm{mV}$. As electrons are gained by thiol residues, they are simultaneously protonated, leading to the tradition of labeling redox active thiol groups as "SH" when reduced or "S-S" when oxidized. DSBs are known to contribute to the stability, activity, and folding of many proteins, including bacterial proteins that are destined to reside outside the cytosol (Kadokura \& Beckwith, 2010). In prokaryotes, this includes outer membrane proteins, flagella, and secreted proteins. This complex but essential process of protein folding and DSB formation is not unique to prokaryotes. Cysteine oxidation also occurs in eukaryotes as proteins are being translocated from the cytosol (reducing compartment) toward the extracellular environment. DSB can also be formed in proteins in the oxidizing environment of the endoplasmic reticulum (Hwang et al., 1992) by PDI, which can oxidize, reduce, and isomerize DSB proteins (Freedman et al., 1994; Luz \& Lennarz, 1996). Oxidases/isomerases accelerate the rate-limiting step of DSB formation by directly catalyzing them or rearranging them. Members of these groups have been well studied in E. coli (as described below). In L. pneumophila, proteins similar to these DSB proteins in E. coli are the subjects of the study presented here. 


\subsection{The DSB System in Escherichia coli}

Almost all organisms, from bacteria to humans, are equipped with enzymes involved in disulfide bond generation and isomerization (Inaba, 2010). These processes have been studied in E. coli (Ito \& Inaba, 2008), the endoplasmic reticulum of yeast (Sevier \& Kaiser, 2008), and the intermembrane space of mitochondria (Chacinska et al., 2009). The discovery of the first bacterial DSB protein (DsbA) happened similarly to any great scientific discovery; discovering something novel while searching for something else entirely. In the case of DsbA discovery, a reporter fusion protein was constructed and subjected to a genetic screen in E. coli, looking for mutations in bacterial secretion. Astonishingly, the reporter protein accidently contained DSB, and the mutants obtained did not interfere with translocation from the cytosol to the periplasm, but mapped to some unknown locus. The mutant was frozen in glycerol for four years until a talented postdoctoral fellow took on the project and proved that the disrupted gene $(d s b A)$ catalyzed the formation of DSBs in the bacterial periplasm (Beckwith, 2007; Bardwell et al., 1991).

Over 250 proteins are expressed by E. coli that contain two or more DSBs and are predicted to enter the periplasm (Hiniker \& Bardwell, 2005; Dutton et al., 2010), and DsbA is thought to interact with many of them (Frech et al., 1996). Since then, a model for DSB formation in the $E$. coli periplasm has been developed from studies conducted in several laboratories. Up until this study the current DSB model, as illustrated in Figure 4, has the periplasmic disulfide oxidoreductase DsbA (Bardwell et al., 1991; Kamitani et al., 1992) as the main enzyme responsible for disulfide bond formation. It is the functional equivalent of PDI that is found in the endoplasmic reticulum of eukaryotes (Freedman et al., 1994). However, DsbA has only one disulfide active site (CXXC) while PDI has two (Luz \& Lennarz, 1996). This 


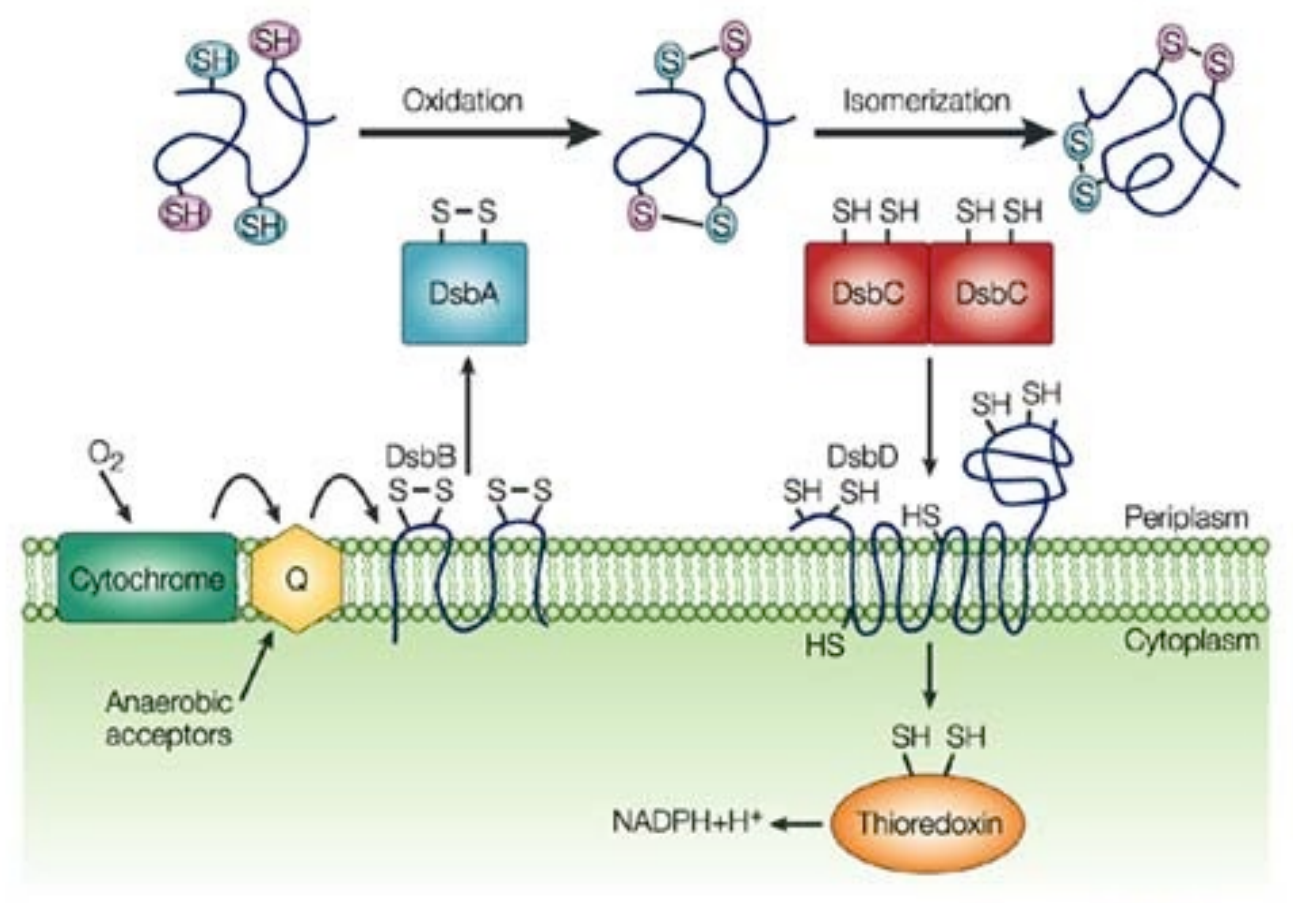

Figure 4. The Disulfide bonds system in E. coli. The oxidative pathway. Disulfides are introduced into substrate proteins by disulfide exchange with DsbA, which is in turn reoxidized by the inner membrane protein DsbB. DsbA is a soluble periplasmic protein while DsbB is an inner membrane protein. The isomerase pathway. Substrates with more than two cysteines may form incorrect disulfides, causing them to misfold. Non-native disulfides are corrected by DsbC. These two proteins are maintained in their active reduced state by the inner membrane protein DsbD. DsbC is a soluble homodimer in the periplasm and DsbD is a membrane protein. S-S indicates cysteines are in the oxidized disulfide bond form while $\mathrm{SH}$ indicates cysteines in the reduced dithiol form. Adapted from Sevier et al., 2002. 
is probably why PDI, a multifunctional protein, is much more active than DsbA in isomerization reactions and does not need to be re-oxidized by another component (Zapun \& Creighton, 1994) while DsbA displays primarily oxidoreductase activity. The redox potential of the active site of DsbA has been measured and is one of the most potent biological oxidants known (Grauschopf et al., 1995). DsbA catalyzes disulfide bond formation by oxidizing the sulfhydryl groups on free cysteine residues of reduced, unfolded polypeptides as soon after they are translocated to the periplasm (see Fig. 4) (Frech et al., 1996; Kadokura \& Beckwith, 2009). Once it has donated its own disulfide bond to its substrate, DsbA itself is reduced. In order to continue its disulfide bond forming function, DsbA need to be re-oxidized. The integral, inner membrane protein DsbB specifically oxidizes DsbA so that it is able to participate in another oxidation reaction (Bardwell et al., 1993; Dailey \& Berg, 1993; Missiakas et al., 1993; Jander et al., 1994). Guilhot et al. (1995) as well as Kishigami et al. (1995) have provided direct evidence of a disulfide-bonded complex between a reduced DsbA and DsbB. In addition, Darby and Creighton (1995) as well as Ruddock et al. (1996) have demonstrated the rate-limiting factor in an oxidation reaction between DsbA and short peptides is the reoxidation of DsbA.

E. coli DsbA is one of the most oxidizing proteins identified in biology (Zapun et al., 1993), with a redox potential of $-122 \mathrm{mV}$ (Carvalho et al., 2006). Its redox potential, coupled with the greater thermodynamic stability in its reduced form, is thought to give rise to its highly oxidizing activity (Philipps \& Glockshuber, 2002). The hydrophobic surface that surrounds the active site of E. coli DsbA aids in binding unfolded proteins, and when this surface is perturbed through mutation, the substrate repertoire of DsbA narrows (Heras et al., 2008; Grimshaw et al., 2008; Vivian et al., 2009). DsbB is an intergral membrane protein that straddles the cytoplasm and periplasm with a four-helix bundle linked through on cytoplasmic and two periplasmic loops 
(Inaba et al., 2004). Each of the periplasmic loops contains a pair of cysteines that act in concert to transfer electrons from DsbA through DsbB onto ubiquinones (Kishigami \& Ito, 1996), which are then reoxidized by terminal oxidases (Bader et al., 2001). The periplasmic loop of DsbB undergoes a conformation change in order to complete the disulfide exchange (Bader et al., 2001). E. coli DsbB has been demonstrated to have a strict substrate specificity for DsbA, and does not participate in direct disulfide bond reactions with reduced nascent polypeptides emerging into the periplasm (Heras et al., 2009).

The DsbA-mediated oxidase system, which is known to introduce disulfide bonds between consecutive cysteine residues in the primary sequence of protein, may not always result in correct DSB formation (Wunderlich \& Glockshuber, 1993), especially in proteins with more than two cysteines, or with proteins that contain non-consecutive disulfide bonds. The isomerase activity of DsbC is required for correct protein folding (Shevchik et al., 1994; Rietsch et al., 1996). DsbC, another periplasmic protein, was first thought to be a disulfide oxidoreductase-like DsbA (Missiakas et al., 1994) because defects in disulfide bond formation of several proteins were seen in $d s b C$ null mutants. However, work demonstrated by Zapun et al. (1995) and supported by Rietsch et al. (1996) revealed that DsbC is actually an isomerase. It is only when it is overexpressed that DsbC can substitute for the loss of DsbA function and can reverse most of the $d s b A^{-}$phenotypic defects (Missiakas et al., 1994). DsbC also has a single disulfide bond Cys-GlyTyr-Cys (CXXC) in each arm of the homodimer (Zapun et al., 1995). In order for DsbC to act as an isomerase, it needs to be in a reduced state. DsbC is kept in a reduced state by its membrane bound redox partner, DsbD (Missiakas et al., 1995; Rietsch et al., 1996). DsbD is a membrane protein with eight transmembrane helices and two periplasmic domains (Katzen \& Beckwith, 2000). DsbC has a C-terminal thioredoxin fold connected to an N-terminal dimerization domain, 
forming a V-shaped homodimer (McCarthy et al., 2000). Dimerization serves two purposes: to form a hydrophobic pocket where misfolded proteins can bind, and secondly to prevent interaction with DsbB (Inaba et al., 2006). This supports the current existing Dsb machinery hypothesis that the oxidative and isomerase pathways are separated to prevent futile cycling (Heras et al., 2009).

\subsection{The Role of DsbA in virulence}

EcDsbA, a $23 \mathrm{kDa}$ periplasmic protein, was first described by Beckwith and Bardwell (Bardwell et al., 1991). Martin et al. (1993) have presented the crystal structure of oxidized DsbA, and demonstrated that structurally it closely resembles the cytoplasmic redox protein thioredoxin despite a low general sequence similarity. The most interesting feature of DsbA is its active site which is composed of two cysteine residues separated by a proline and a histidine, which form a very unstable and reactive disulfide bond (Wunderlich \& Glockshuber, 1993; Zapun et al., 1994). It is the instability of this bond that facilitates the very rapid transfer of DSB to newly translocated polypeptide substrates during the oxidation reaction (Darby \& Creighton, 1995; Frech et al., 1996). The active site of DsbA is surrounded by a relatively extensive uncharged hydrophobic surface, which is proposed to be a substrate-binding domain (Martin et al., 1993; Guddat et al., 1997), where substrates would bind and be stabilized prior to transfer of disulfide bonds.

DSB proteins, especially DsbA-like proteins, play a role in the biogenesis of virulence factors in many bacteria (Yu \& Kroll, 1999; Lasica et al., 2007). Even though DsbA is not essential for E. coli survival, mutations in the $d s b A$ gene can result to loss of virulence or cause more pleiotropic effects such as loss of motility, reduced alkaline phosphatase activity, reduced OmpA 
and $\beta$-lactamase proteins, increased $\beta$-galactosidase activity, increased sensitivity to DTT and inability to assemble F pili (Bardwell et al., 1991; Kamitani et al., 1992; Missiakas et al., 1993). It has been also suggested that DsbA might have a chaperone-like activity that is independent of its disulfide oxidoreductase activity (Jacob-Dubuisson et al., 1994; Zheng et al., 1997). In Gramnegative bacteria, fimbriae are classified by structure (type I, type P, and type IV (known as bundle-forming pili)) or how they are assembled (chaperone usher pathway or type IV secretion) (Fronzes et al., 2009a). These macromolecular structures require DsbA activity in order to have their individual subunits properly formed and assembled correctly in the periplasm. Okamoto et al. (1995) have demonstrated that DsbA is also involved in the DSB formation of two E. coli heat-stable enterotoxins, which cause increased intestinal secretion and diarrhea. They demonstrated that in a $d s b A$ mutant, these extracellular toxins are degraded before they reach the extracellular medium. However, disulfide bond formation is not completely abolished in a $d s b A^{-}$ strain. Overexpression of DsbC, an isomerase, was found to functionally substitute for a loss of DsbA function and DsbC-mediated disulfide bond formation could therefore be responsible for the residual activity seen (Missiakas et al., 1994). Similar bifunctional activity of a DsbA-like protein (DsbA2) in L. pneumophila will be further described in this study. The above combined studies have suggested the importance of $\mathrm{DsbA}$ in disulfide bond formation in some virulence factors.

\subsection{DsbA homologues in other microorganisms}

Since the discovery of DsbA in E. coli and the definition of its role in the stability and activity of many extracytoplasmic proteins, DsbA homologues have been described in other pathogens, some of which will be described in this section. First, DsbA was shown to be involved in the 
biogenesis of Vibrio cholerae enterotoxins (Yu et al., 1992; Yu et al., 1993). Pectate lyase and the cellulose EGZ, both enzymes catalyzing plant cell wall degradation, were rapidly degraded in the periplasm of a $d s b A$ mutant in Erwinia chrysanthemi (recently renamed as Dickeya dadantii) (Shevchik et al., 1995). Watarai et al. (1995) also demonstrated that in Shigella flexneri, DsbA is required for Spa32 to fold correctly. Spa32 is an outer membrane protein involved in the release of Ipa proteins that are essential for the organism to be able to invade epithelial cells. A DsbA homologue has also been found in the Gram-positive bacterium Bacillus brevis (Ishihara et al., $1995)$ that complements an E. coli $d s b A^{-}$strain. It was proposed that this DsbA homologue facilitates disulfide bond formation, probably on the cell surface during or immediately after translocation of the substrate proteins across the cytoplasmic membrane. Also, homologs have also been found in Pseudomonas aeruginosa (Leipelt et al., 1997), Azobacter vinelandii (Ng et al., 1997), Klebsiella oxytoca (Baek et al., 1996), Lpn (Sadosky \& Shuman, 1994), and in many other pathogens. The overall protein sequence of many of the homologues is highly similar, and the region around the active site $(\mathrm{CXXC})$ is the most well conserved area among all disulfide oxidoreductase that have been identified to date.

Lpn and many Gram-negative microorganisms other than E. coli contain more than one DsbA-like protein, but their functions are yet to be properly characterized. Most importantly, no DsbA homologue has been reported to have both oxidase and isomerase functions for disulfide bond formation in proteins prior to this study in Legionella pneumophila. 


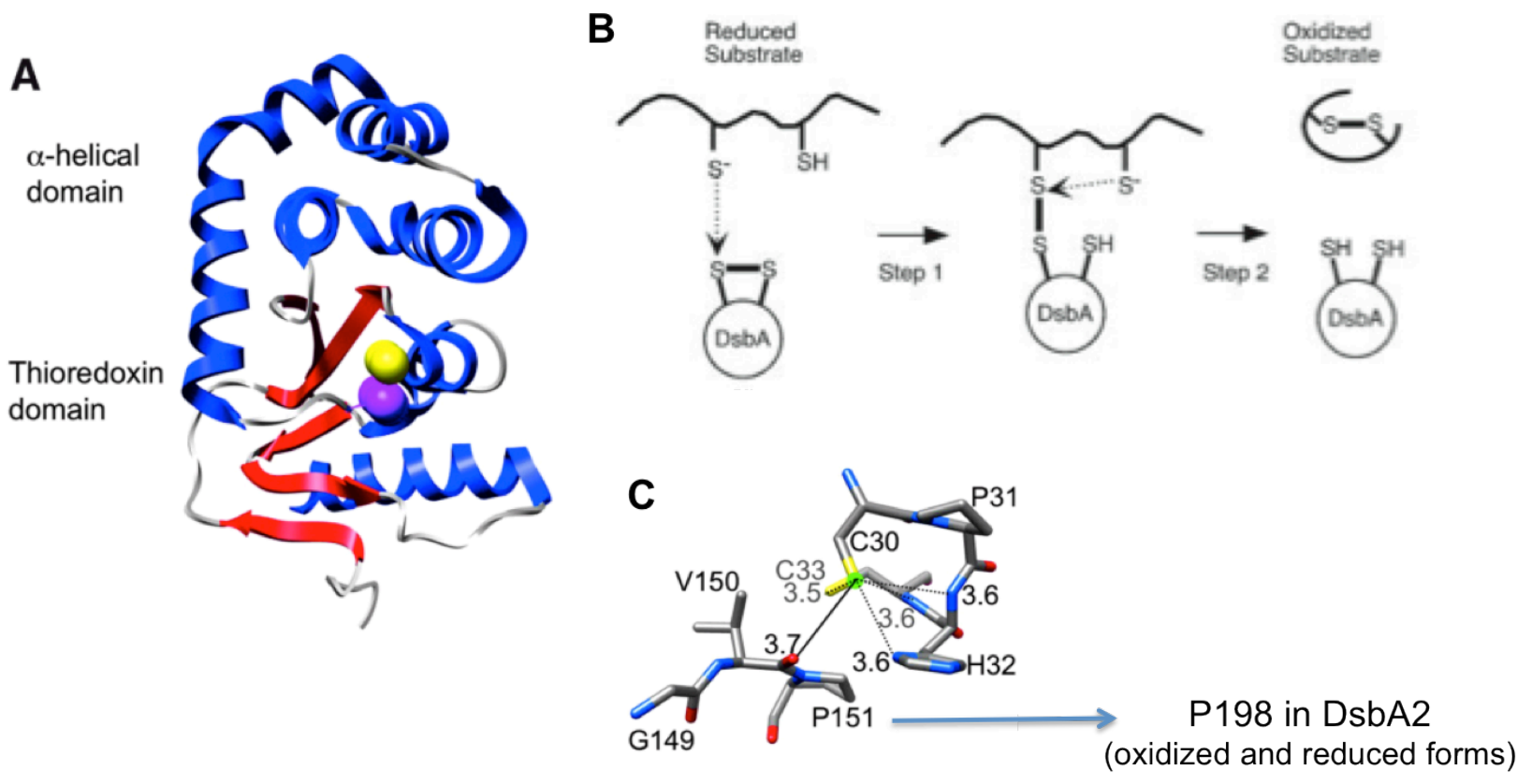

Figure 5. Oxidation of substrate protein by DsbA. A. Ribbon representation of E. coli DsbA structure (PDB accession number, 1FVK) generated by PyMol. Yellow spheres represent sulfurs of active site cysteines. Purple spheres also indicate Pro31 and Pro151 residues. B. Diagram of DsbA interacting with substrates via $S^{N} 2$ mechanism adapted from Kadokura et al. (2004). C. Active site showing cysteine residue and cis-proline that was changed to threonine, which causes a significant defect in resolution of DsbA-substrate complexes. The arrow indicates the conserved proline at position-198 in DsbA2 of Lpn. 


\subsection{Dsb Machinery in Legionella pneumophila}

Disulfide bonds, both intermolecular and intramolecular, provide structure and rigidity to macromolecular protein complexes such as chaperone/usher pili (Heras et al., 2009), flagella motors (Bardwell et al., 1991; Zhang \& Donnenberg, 1996), dimeric proteins such as alkaline phosphatase (Bardwell et al., 1991), type III secretion systems (Heras et al., 2009), type IV secretion systems (Jameson-Lee et al., 2011), and many toxins including cholera and pertussis toxins (Peek \& Taylor, 1992; Zhang \& Donnenberg, 1996; Stenson \& Weiss, 2002; Kadokura \& Beckwith, 2010; Ruiz et al., 2010). In L. pneumophila, many membrane-spanning and surfaceexpressed structural proteins such as the OmpS porin, a trimer that is held together by inter-chain DSBs (Hoffman et al., 1992), and several Dot/Icm core proteins have disulfide bonds (Conover et al., 2003).

Like many pathogens that commonly cause disease in humans, animals, and plants and express a T4SS, Legionella contains two DsbA-like proteins, namely DsbA2, which is reported to be essential for virulence and the proper assembly of the Dot/Icm T4SS system (Table 1) (Kpadeh et al., 2013), and DsbA1, a second nonessential DSB oxidoreductase.

DsbA proteins with point mutations have shown deficiencies in substrate translocation (Sexton et al., 2004). The process of introducing DSBs into newly translocated proteins is thought to occur via formation of mixed disulfide complexes between DsbA-like protein and its substrates. However, these complexes are transient. Kadokura et al (2004) showed that it is possible to detect covalent intermediates in vivo by a mutation in DsbA that alters cis proline-151 (Fig. 5). Using this mutant they were able to identify substrates of DsbA. Knowing that the cisproline is highly conserved among thioredoxin superfamily members, a cis-proline mutant of DsbA2 in Lpn was also constructed as described by (Jameson-Lee et al., 2011), and used to 
capture substrate proteins and to evaluate bacterial virulence. Substrates captured by DsbA2 P198T mutant revealed that DsbA2 could function as a disulfide bond oxidoreductase for outer membrane proteins and other virulence factors (Jameson-Lee et al., 2011).

DsbA2 exists as a mixture of oxidized and reduced forms in vivo in Legionella (UVA thesis, 2012; Kpadeh et al., 2013), and is reported to be similar to Coxiella burnetii outer membrane protein 1 (Com1) (Hendrix et al., 1993; Jameson-Lee et al., 2011). Prior to this study, Legionella dsbA1 had no discernable phenotype (Jameson-Lee et al., 2011). Legionella DsbB1 and DsbB2 (oxidant of the oxidase (s)), and DsbD1 and DsbD2 (reductant of the disulfide bond isomerase) have been discovered in screens involving the Dot/Icm T4SS (Conover et al., 2003), but not further characterized.

The question of whether or not the two DsbAs (DsbA1 and DsbA2) are required for disulfide bond formation in the periplasm still remained. In this study, we sought to characterize the bifunctional activities of DsbA2, and identify the functional contributions of the DSB proteins in Legionella pneumophila for proper assembly of virulence factors, such as the Dot/Icm T4SS. 
Table 1. Disulfide bond composition of genera expressing DsbA2

\begin{tabular}{|lcccccccc|}
\hline Bacteria & DsbA1 & DsbA2 & DsbB & DsbD & CcmG & DsbC/G T4SDS \\
\hline Legionella & + & + & + & + & + & - & + \\
Rickettsia & + & + & + & + & + & - & + \\
Brucella & + & + & + & + & + & - & + \\
Anaplasma & + & + & + & + & + & - & + \\
Agrobacterium & + & + & + & + & + & - & + \\
Coxiella & - & + & + & + & - & - & + \\
Ehrlichia & + & + & + & + & + & - & + \\
Afipia & + & + & + & + & + & - & + \\
Rhodobacter & + & + & + & + & + & - & - \\
Rhodopseu. & + & + & + & + & + & - & - \\
Bradyrhizobium & + & + & + & + & + & - & - \\
Azospirillum & + & + & + & + & + & - & - \\
Bartonella & + & + & + & + & + & - & + \\
Caulobacter & + & + & + & + & + & - & - \\
Rhodospirillum & + & + & + & + & + & - & - \\
Aeromonas & + & + & + & + & + & - & - \\
Bordetella & + & - & + & + & + & + & + \\
Escherichia & + & - & + & + & + & + & - \\
\hline
\end{tabular}

This is a partial list from more than 60 genomes identified by BLASTP with the 27-amino-acid dimerization domain sequences of DsbA in Legionella pneumophila. The E. coli protein sequences were used for the searches against each genus of DsbA1, DsbB, DsbD, DsbC, DsbG, and CcmG (a thioredoxin-like periplasmic enzyme associated with cytochrome $c$ maturation). The presence or absence of a T4SS was determined from the literature, and the question mark for Francisella tularensis indicated that the system might be incomplete (Kpadeh et al., 2013). 


\subsection{Objectives of study}

The proper formation of disulfide bonds in bacterial proteins outside of the cytoplasm is aided by specific oxidoreductase, first characterized in E. coli as the disulfide bond (DSB) family of proteins. The DSB enzymes are part of the thioredoxin (TRX) super family of proteins, which are defined by the presence of one or more thioredoxin folds and have been described in detail in the above sections. Briefly, these enzymes participate in disulfide bond formation through a conserved Cys- $\mathrm{x}-\mathrm{x}-\mathrm{Cys}(\mathrm{CxxC})$ active site motif, and have evolved to either reduce or oxidize target substrates. Our interest in disulfide bond formation in L. pneumophila is based on our discovery that the major outer membrane protein trimer is cross-linked by inter-chain disulfide bonds. In addition, ultrastructural studies of the cyst form indicated substantial remodeling of the cell envelope that included changes to these outer membrane proteins (Anfinsen et al., 1961; Anfinsen \& Haber, 1961; Bardwell et al., 1991). Much of the cell remodeling occurs extracytoplasmically. Therefore, we became interested in investigating DSB proteins that participate in this process.

Over the last decade, a sophisticated system for disulfide bond formation and protein folding in the periplasm of Gram-negative gammaproteobacteria has been described using E. coli as a model. However, it is now known that the complement of Dsb proteins in E. coli is the "classical" pathway (mostly referred to as the canonical model (Fig. 4)), but not conserved universally (Dutton et al., 2008). Many bacterial species have diversified from the canonical model, as bioinformatic and phylogentic analyses indicate that all Gram-negative species expressing T4SS (exception: Bordetella pertussis) express DsbA2 and lack orthologues of DsbC and DsbG (see Table 1). DsbA2 is highly conserved in intracellular human, animal and plant pathogens that employ T4SS for virulence and in most aquatic and soil bacteria, many of which 
are also dimorphic. Our lab recently identified DSB oxidoreductase DsbA2. In addition to its essential functions, it seemed to be required for proper assembly or function of the T4SS. Also, previous studies showed DsbA2 to exist as a mixture of oxidized and reduced forms in the periplasm of Lpn. A DsbA2P198T mutant protein was used to capture substrates, supporting that DsbA2 was a DsbA oxidase (Jameson-Lee et al., 2011). The existence of a mixture of oxidized and reduced forms suggests that DsbA2 is a naturally occurring bifunctional DSB oxidoreductase, thus suggesting an "alternative" DSB pathway (Fig. 21). In addition, in E. coli DsbA is a monomer while DsbC and DsbG are dimers. Because DsbG does not complement isomerase activity in an E. coli $d s b C$ mutant (Ren \& Bardwell, 2011) this study did not explore the relationship of Lpn DsbA2 with DsbG. The major structural difference between DsbA and DsbC in E. coli is the presence of a dimerization domain on $\mathrm{DsbC}$, which enables DsbC to exist as a homodimer and function as a protein disulfide isomerase.

Prior to this study, the $d s b A 2$ allele, previously known as Com1, was of unknown function and had not been studied in any bacteria, other than as a diagnostic antigen for C. burnetii (Hendrix et al., 1993), and by Dr. Mann in Francisella where the DsbA2 is not essential for viability, but is for virulence (Qin et al., 2011). In this study, we sought to characterize this novel DsbA-like oxidoreductase (DsbA2). We will demonstrate the following: i) DsbA exists as a homodimer and also functions as a protein disulfide isomerase, ii) deletion of DsbA2 dimerization domain results in a monomeric DsbA2 that exhibits oxidase activity, and is important for proper assembly and function of the T4SS by functional assays (invasion of HeLa cells, intracellular replication in amoeba, and contact-dependent lysis of RBC), iii) reconstitution of Lpn $d s b$ genes in an E. coli null mutant reveal that DsbA2 is maintained as a mixture of $\mathrm{SH} / \mathrm{S}-\mathrm{S}$, exhibiting 
oxidase activity using soft agar motility assay, iv) and the DsbDs of Lpn show considerable divergence from $E$. coli DsbD, both structurally and functionally.

In addition to DsbA2, Legionella contains DsbA1, which is dispensable for intracellular multiplication. We sought to investigate the differences in conformation, and expression profile of DsbA1 and DsbA2 in L. pneumophila, and their ability to complement E. coli mutants. We also crystallized DsbA1 and then structurally and functionally compared it to E. coli DsbA. The results presented in this study will further broaden our understanding of the $d s b$ genes in Legionella and reveal that the "DsbA2 system" differs fundamentally from the DsbA/DsbC system of E. coli, challenging the established paradigms. 


\section{Chapter 2. Materials and Methods}

\subsection{Bacteriological techniques}

The bacterial strains and the cloning vectors used in this study are listed in Table 2 . Bacterial stock cultures were maintained at $-85^{\circ} \mathrm{C}$ in nutrient broth containing $10 \%$ dimethyl sulfoxide (DMSO) or glycerol.

\subsubsection{E. coli}

The Escherichia coli strains were grown on either Luria-Bertani (LB) agar or LB broth (per 1 liter of distilled water: $10 \mathrm{~g}$ yeast extract, $10 \mathrm{~g}$ tryptone, $5 \mathrm{~g}$ sodium chloride; for agar also add 1.5\% bacto agar). E. coli strains RGP443, RGP663, RGP665, RGP665, RGP810, JCB570, and JCB571 were generously provided by Dr. James C. Bardwell and Dr. Ren Guoping (University of Michigan, Ann Habor, MI). Cells of these strains were grown at $37^{\circ} \mathrm{C}$ under aerobic conditions in appropriate media supplemented with the appropriate antibiotics. Proper antibiotic (Sigma-Aldrich) selection for E. coli strains was maintained when appropriate, as follows: streptomycin $(100 \mu \mathrm{g} / \mathrm{ml})$, kanamycin $(40 \mu \mathrm{g} / \mathrm{ml})$, gentamicin $(10 \mu \mathrm{g} / \mathrm{ml})$, chloramphenicol $(20 \mu \mathrm{g} / \mathrm{ml})$, metronidazole $(20 \mu \mathrm{g} / \mathrm{ml})$ and ampicillin $(100 \mu \mathrm{g} / \mathrm{ml})$. For transformation of pBlueScript-derived vectors, $40 \mu 1$ of a $20 \mathrm{mg} / \mathrm{ml}$ solution of 5-bromo-4-chloro3-indolyl- $\beta$-D-galactopyronidase (X-gal) or isopropyl- $\beta$-D-galactoside (IPTG) was added to a final concentration of $1 \mathrm{mM}$. 


\subsubsection{Legionella pneumophila}

L. pneumophila strains were grown at $37^{\circ} \mathrm{C}$ on buffered charcoal yeast extract (BCYE) or in buffered yeast extract (BYE) broth: $10 \mathrm{~g}$ yeast extract, $1 \mathrm{~g}$ [2-(2-amino-2-oxoethyl)-amino] ethane sulfonic acid (ACES), and $1 \mathrm{~g} \alpha$-ketoglutaric acid, with $\mathrm{pH}$ adjusted to 6.6-6.7 with $6 \mathrm{~N}$ potassium hydroxide. BCYE solid medium included all components of BYE broth as well as $16 \mathrm{~g} / 1$ agar and $1.5 \mathrm{~g} / 1$ of activated charcoal. After autoclaving, all media received $0.4 \mathrm{~g} / 1 \mathrm{~L}-$ cysteine $\mathrm{pH}$ 6.6-6.7, $0.25 \mathrm{~g} / 1$ ferric pyrophosphate and $100 \mu \mathrm{g} / \mathrm{ml}$ thymidine. Where appropriate, media were supplemented as follows: streptomycin $(100 \mu \mathrm{g} / \mathrm{ml})$, kanamycin $(40 \mu \mathrm{g} / \mathrm{ml})$, gentamicin $(10 \mu \mathrm{g} / \mathrm{ml})$, chloramphenicol $(20 \mu \mathrm{g} / \mathrm{ml})$, metronidazole $(20 \mu \mathrm{g} / \mathrm{ml})$ and ampicillin $(100 \mu \mathrm{g} / \mathrm{ml})$.

\subsubsection{Culture conditions}

Starter cultures from single colonies or diluted overnight cultures were prepared as previously described (LeBlanc et al., 2006) and used to inoculate pre-warmed BYE or LB to an optical density at $600 \mathrm{~nm}\left(\mathrm{OD}_{600}\right)$ of 0.2 . For growth curve determinations, $1 \mathrm{ml}$ aliquot samples were taken every $2 \mathrm{~h}$ (triplicate) and optical density was determined at $600 \mathrm{~nm}$ every 30 min until stationary phase was reached.

\subsubsection{Determination of bacterial optical density}

Bacterial optical density (OD) was determined at $600 \mathrm{~nm}\left(\mathrm{OD}_{600}\right)$ using a Unico UV-2100 spectrophotometer. For agar plate grown bacteria, cells were resuspended in $1 \mathrm{ml}$ of phosphatebuffered saline (PBS) [137mM NaCl, $2.7 \mathrm{mM} \mathrm{KCl,} 10 \mathrm{mM} \mathrm{Na} 2 \mathrm{HPO}_{4}$, and $\left.2 \mathrm{mM} \mathrm{KH}_{2} \mathrm{PO}_{4}\right]$ or 
appropriate culture medium, and vortexed until a homogenous suspension is obtained. Samples were diluted as required, transferred to a cuvette, and $\mathrm{OD}_{600}$ was determined. For broth cultures, one hundred microliters of this suspension were then removed and added to $900 \mu \mathrm{l}$ of $\mathrm{ddH}_{2} \mathrm{O}$ or appropriate media (1/100 dilution). The diluted sample was then mixed by inversion, transferred to a cuvette, and the optical density determined at $600 \mathrm{~nm}$. An $\mathrm{OD}_{600}$ of 1.0 of L. pneumophila was found to be equivalent to $1 \times 10^{9} \mathrm{CFU}$.

\subsection{Molecular biology techniques}

\subsubsection{Construction of P198T-His 6 mutants}

To create mutant alleles of DsbA1 or DsbA2, proline at position 150 or 198 was changed to theorine using a PCR-based strategy with primer pairs P150T-F and P150T-R, or P198T-F and P198-R, respectively. The PCR amplicons were digested with EcoRI/ BamHI and cloned into pMMB206 to enable expression under a controllable promoter (lac $I^{q}$ - and IPTG-inducible promoter) (Morales et al., 1991). For both P150T and P198T constructs, a His $6_{6}$-tag was also engineered by PCR. All constructs were confirmed by PCR, DNA sequencing and introduced into the Lp02 and AA100 strains by electroporation or by natural transformation. 
Table 2. Bacterial strains and plasmids used in this study

\begin{tabular}{|c|c|c|}
\hline Bacterial Strain or plasmid & Relevant properties & Reference or source \\
\hline \multicolumn{3}{|l|}{ Legionella pneumophila } \\
\hline AA100 & $\begin{array}{l}\text { Clinical isolate from Los Angeles, } \\
\text { CA. Also called } 130 \mathrm{~b}\end{array}$ & Yousef Abu Kwaik \\
\hline $\mathrm{AA} 100 \Delta d s b A 1$ & AA100 dsbA1 mutant & This study \\
\hline LpO2 & $\begin{array}{l}\text { Philadelphia-1 derivative, } r p s L \\
h s d R \text { thy } A\left(\mathrm{Sm}^{\mathrm{R}}\right)\end{array}$ & Berger \& Isberg \\
\hline$A A 100 \Delta \operatorname{dot} A$ & AA100 $\operatorname{dot} A$ mutant & Yousef Abu Kwaik \\
\hline \multicolumn{3}{|l|}{ Escherichia coli } \\
\hline $\mathrm{DH} 5 \alpha$ & 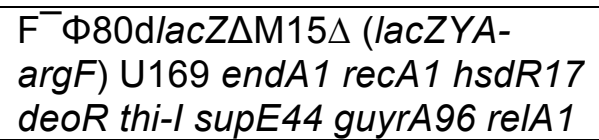 & Clontech \\
\hline BL21 Codon Plus & $\begin{array}{l}\mathrm{F}^{-} \text {ompT hsdS }\left(\mathrm{rB}^{-} \mathrm{mB}^{-}\right) \text {dcm }^{+} \mathrm{Tet}^{\mathrm{R}} \\
\text { gal }(\mathrm{DE} 3) \text { endA The [argU ileY } \\
\text { leuW] }\left(\mathrm{Cm}^{\mathrm{R}}\right)\end{array}$ & Stratagene \\
\hline JCB570 & E. coli wild type strain & James Bardwell \\
\hline JCB571 & JCB570 $\Delta d s b A ; \operatorname{Kan}^{R}$ & James Bardwell \\
\hline R P4 5 & E. coli JP114 dsbB- & Ren \& Bardwell \\
\hline RGP438 & E. coli JP114 $\Delta d s b A \Delta d s b B$ & Ren \& Bardwell \\
\hline RGP209 JP114 dsbC- & E. coli JP114 dsbC- & Ren \& Bardwell \\
\hline $\begin{array}{l}\text { RGP663:JP114 + PDI } \\
\text { detector }\end{array}$ & $\begin{array}{l}\text { JP114 + PDI detector, Tet and } \\
\text { Amp resistance. Amp resistance } \\
\text { goes to } 3 \mathrm{~g} / \mathrm{L}\end{array}$ & Ren \& Bardwell \\
\hline $\begin{array}{l}\text { RGP665: RGP209 PDI } \\
\text { etector }\end{array}$ & $\begin{array}{l}\text { Tet and Amp resistance (both } \\
\text { from the plasmid of PDI detector). } \\
\text { However, the Amp resistance is } \\
\text { weak as } 0.2 \mathrm{~g} / \mathrm{L} \text {. }\end{array}$ & Ren \& Bardwell \\
\hline RGP666 & $\begin{array}{l}\text { E. coli JP114 } \Delta d s b D+\mathrm{pPDI} \\
\text { detector, Tet and Amp resistance }\end{array}$ & Ren \& Bardwell, \\
\hline R P8 0 & $\begin{array}{l}\text { E. coli JP114 } \Delta d s b C \Delta d s b D+ \\
\text { pPDI detector, Tet, Amp and Kan } \\
\text { resistance }\end{array}$ & Ren \& Bardwell, \\
\hline $\begin{array}{l}\text { RGP666: pBS:LpndsbD1+ } \\
\text { PDI detector }\end{array}$ & $\begin{array}{l}\text { Tet, Amp (both from the plasmid } \\
\text { of PDI detector) and Cm (from } \\
\text { pBS). }\end{array}$ & This study \\
\hline $\begin{array}{l}\text { RGP666:pBS:LpndsbD2 + } \\
\text { PDI detector }\end{array}$ & $\begin{array}{l}\text { Tet, Amp (bot from the plasmid } \\
\text { of DI detector) and Cm (from } \\
\text { pBS). }\end{array}$ & This study \\
\hline $\begin{array}{l}\text { RGP666: pBS:LpndsbD1 + } \\
d s b A 2+\text { PDI detector }\end{array}$ & $\begin{array}{l}\text { Tet, Amp (both from the plasmid } \\
\text { of PDI detector) and Cm (from } \\
\text { pBS). DsbD1 and DsbA2 are } \\
\text { under the same promoter in the } \\
\text { pBS vector. }\end{array}$ & This study \\
\hline $\begin{array}{l}\text { RGP666: pBS:LpndsbD2 + } \\
d s b A 2+\text { PDI detector }\end{array}$ & $\begin{array}{l}\text { Tet, Amp (both from the plasmid } \\
\text { of PDI detector) and Cm (from } \\
\text { pBS). DsbD2 and DsbA2 are } \\
\text { under the same promoter in the }\end{array}$ & This study \\
\hline
\end{tabular}




\begin{tabular}{|c|c|c|}
\hline & pBS vector. & \\
\hline RGP810 JP114 $d s b C-d s b D-$ & $\begin{array}{l}\text { E. coli JP114 } \Delta d s b C \Delta d s b D+ \\
\text { pPDI detector, Tet, Amp and Kan } \\
\text { resistance }\end{array}$ & $\mathrm{R} n \&$ Bardwell \\
\hline $\begin{array}{l}\text { RGP810: pBS:LpndsbD1+ } \\
\text { PDI detector }\end{array}$ & $\begin{array}{l}\text { Tet, Kan and Amp (from the } \\
\text { plasmid of PDI detector) and } \mathrm{Cm} \\
\text { (from pBS). }\end{array}$ & This study \\
\hline $\begin{array}{l}\text { RGP810:pBS:LpndsbD2 + } \\
\text { PDI detector }\end{array}$ & $\begin{array}{l}\text { Tet, Kan and Amp (from the } \\
p \text { asmid of PDI detector) and } \mathrm{Cm} \\
\text { (from pBS). }\end{array}$ & This study \\
\hline $\begin{array}{l}\text { RGP810: pBS:LpndsbD1 + } \\
\text { dsbA2 + PDI detector }\end{array}$ & $\begin{array}{l}\text { Tet, Kan and Amp (from the } \\
\text { plasmid of PDI detector) and } \mathrm{Cm} \\
\text { (from pBS). DsbD1 and DsbA2 } \\
\text { are under the same promoter in } \\
\text { the pBS vector. }\end{array}$ & This study \\
\hline $\begin{array}{l}\text { RGP810: pBS:LpndsbD2 + } \\
\text { dsbA2 + PDI detector }\end{array}$ & $\begin{array}{l}\text { Tet, Kan and Amp (from the } \\
\text { plasmid of PDI detector) and } \mathrm{Cm} \\
\text { (from pBS). DsbD2 and DsbA2 } \\
\text { are under the same promoter in } \\
\text { the pBS vector. }\end{array}$ & This study \\
\hline ZK01 & $\begin{array}{l}\text { RGP438 + LpndsbD } 1^{\mathrm{C}}\left(\text { Gent }^{\mathrm{R}},\right. \\
\left.\mathrm{Kan}^{\mathrm{R}}, \mathrm{Amp}^{\mathrm{R}}\right)\end{array}$ & This study \\
\hline ZK02 & $\begin{array}{l}\text { RGP438+ LpndsbD2 }{ }^{\mathrm{C}}\left(\mathrm{Gent}^{\mathrm{R}} \text {, }\right. \\
\left.\operatorname{Kan}^{\mathrm{R}}, \mathrm{Amp}^{\mathrm{R}}\right)\end{array}$ & This Study \\
\hline ZK01::pA2B1 & $\begin{array}{l}\text { ZK01+dsbA2 and dsbB1 } \\
\text { domains in pMMB206 }\left(\mathrm{Cm}^{\mathrm{R}},\right. \\
\left.\text { Gent }^{\mathrm{R}}, \mathrm{Kan}^{\mathrm{R}}, \mathrm{Amp}^{\mathrm{R}}\right)\end{array}$ & This Study \\
\hline ZK01::pA2B2 & $\begin{array}{l}\text { ZK01 + dsbA2 and } d s b B 2 \\
\text { domains in pMMB206 }\left(\mathrm{Cm}^{\mathrm{R}},\right. \\
\left.\mathrm{Gent}^{\mathrm{R}}, \mathrm{Kan}^{\mathrm{R}}, \mathrm{Amp}^{\mathrm{R}}\right)\end{array}$ & This Study \\
\hline ZK02::pA2B2 & $\begin{array}{l}\text { ZK02 + dsbA2 and } d s b B 2 \\
\text { domains in pMMB206 }^{2}\left(\mathrm{Cm}^{\mathrm{R}} \text {, }\right. \\
\left.\text { Gent }^{R}, \mathrm{Kan}^{\mathrm{R}}, \mathrm{Amp}^{\mathrm{R}}\right)\end{array}$ & This Study \\
\hline \multicolumn{3}{|l|}{ Plasmids } \\
\hline pBlueScript II KS (+) & Cloning vector, $A m p^{R}$ & Stratagene \\
\hline $\mathrm{pBC}$ & Cloning vector, $\mathrm{Cm}^{R}$ & Stratagene \\
\hline $\mathrm{pBC}:: d s b A 1$ & $\mathrm{pBC}$ containing $d s b A 1$ sequence & This study \\
\hline $\mathrm{pBC}:: d s b A 1$ P150T & $\begin{array}{l}\text { pBC containing dsbA1 P150T } \\
\text { point mutation sequence using } \\
\text { quick change site-directed } \\
\text { mutagenesis strategy. }\end{array}$ & This study \\
\hline pMMB206 & $\begin{array}{l}\text { Derivative of pRSF1010; Ptac } \\
\text { promoter and IPTG-inducible } \\
\text { lac/Q system; Cm }\end{array}$ & Morales \\
\hline pBH6119 & $\begin{array}{l}\text { Promoterless green fluorescent } \\
\text { protein (GFP) vector; } \mathrm{Amp}^{\mathrm{R}} \text {, Thy }\end{array}$ & Hammer \& Swanson \\
\hline pMMB206::dsbA2 & $\begin{array}{l}\text { pMMB206 containing } d s b A 2 \\
\text { coding sequence }\end{array}$ & This study \\
\hline pMMB206::dsbA1 & pMMB206 containing $d s b A 1$ & This study \\
\hline
\end{tabular}




\begin{tabular}{|c|c|c|}
\hline & coding sequence & \\
\hline pMMB206::dsbA1 P150T & $\begin{array}{l}\text { pMMB206 containing } d s b A 1 \\
P 150 T \text { coding sequence }\end{array}$ & This study \\
\hline pMMB206::dsbA1 P150T-His6 & $\begin{array}{l}\text { pMMB206 containing } d s b A 1 \\
P 150 T \text { coding sequence with C- } \\
\text { terminal His-tagged expression } \\
\text { sequence. }\end{array}$ & This study \\
\hline pMMB206::dsbA2 P198T & $\begin{array}{l}\text { dsbA2 P198T mutant in } \\
\text { pMMB206 cloning vector }\end{array}$ & This Study \\
\hline pMMB206::dsbA2 $\Delta N$ & $\begin{array}{l}\text { dsbA2 minus dimerization domain } \\
\text { in pMMB206 }\end{array}$ & This Study \\
\hline pMMB206::dsbA2 $\Delta N$ P198T & $\begin{array}{l}d s b A 2 \Delta N \text { P198T mutant in } \\
\text { pMMB206 }\end{array}$ & Jameson-Lee \\
\hline pMMB206dsbA2::dsbB1 & $\begin{array}{l}d s b A 2 \text { and } d s b B 1 \text { domains in } \\
\text { pMMB206 }\end{array}$ & This Study \\
\hline pMMB206dsbA2:: dsbB2 & $\begin{array}{l}d s b A 2 \text { and } d s b B 2 \text { domains in } \\
\text { pMMB206 }\end{array}$ & This Study \\
\hline pMMB206dsbA2:::dsbD1 & $\begin{array}{l}\text { dsbA2 and } d s b D 1 \text { domains in } \\
\text { pMMB206; co-express under the } \\
\text { same Ptac promoter and IPTG- } \\
\text { inducible lac/Q system; } \mathrm{Cm}^{\mathrm{R}}\end{array}$ & This Study \\
\hline pMMB206dsbA2::dsbD2 & $\begin{array}{l}\text { dsbA2 and } d s b D 2 \text { domains in } \\
\text { pMMB206; co-express under the } \\
\text { same Ptac promoter and IPTG- } \\
\text { inducible lac/Q system; } \mathrm{Cm}^{\mathrm{R}}\end{array}$ & This Study \\
\hline pMMB206dsbD1 & $d s b D 1$ domain in pMMB206 & This Study \\
\hline pMMB206dsbD2 & $d s b D 2$ domain in pMMB206 & This Study \\
\hline $\mathrm{pBC} d s b B 1$ & $d s b B 1$ in $\mathrm{pBC}$ cloning vector & This Study \\
\hline $\mathrm{pBC} d s b B 2$ & $d s b B 2$ in $\mathrm{pBC}$ cloning vector & This Study \\
\hline $\mathrm{pBC} d s b A 2 \Delta N$ & $\begin{array}{l}\text { dsbA2 minus dimerization domain } \\
\text { in } \mathrm{pBC}\end{array}$ & This Study \\
\hline pMMB206:: Empty vector & $\begin{array}{l}\text { AA100 containing pMMB206 } \\
\text { empty vector }\end{array}$ & This study \\
\hline pRDXA & $\begin{array}{l}\text { Dual suicide vector pBOC20 with } \\
B . \text { subtilis sacB (conferring } \\
\text { sucrose sensitivity) and } H \text {. pylori } \\
r d x A \text {; nitro-reductase, as counter } \\
\text { selectable markers (conferring } \\
\text { sensitivity to metronidazole); } \mathrm{Cm}^{\mathrm{R}}\end{array}$ & $\begin{array}{l}\text { Ann Karen C. } \\
\text { Brassinga }\end{array}$ \\
\hline pRDXA $\triangle d s b A 1::$ Gent & $\begin{array}{l}\text { dsbA1::Gent suicide delivery } \\
\text { vector }\end{array}$ & This study \\
\hline $\begin{array}{l}\text { pRDXA } \triangle d s b A 1: \\
\text { pMMB206::dsbA1 P150T }\end{array}$ & $\begin{array}{l}\text { Gent (from the knockout plasmid) } \\
\text { and Cm from the pMMB206 } \\
\text { plasmid. }\end{array}$ & This study \\
\hline $\begin{array}{l}\text { pBRDX } \Delta d s b A 1: \\
\text { pMMB206::dsbA1 P150T-His } 6\end{array}$ & $\begin{array}{l}\text { Gent (from the knockout plasmid) } \\
\text { and Cm from the pMMB206 } \\
\text { plasmid. Plus c-terminal his- } \\
\text { tagged expressing sequence. }\end{array}$ & This study \\
\hline
\end{tabular}




\begin{tabular}{|l|l|l|}
\hline PDI detector & Beta-lactamase with engineered & Ren \& Bardwell \\
& non-consecutive disulfide bond & \\
(Ren \& Bardwell, 2011). Requires & & \\
& disulfide isomerase for proper & \\
folding and Amp resistance. & \\
\hline
\end{tabular}

$A m p^{R}, \operatorname{Kan}^{R}$, Gent $^{R}$, Strep ${ }^{R}$, and $\mathrm{Cm}^{R}$ indicate resistance markers to ampicillin, kanamycin, gentamicin, streptomycin, and chloramphenicol, respectively. $\mathrm{Thy}^{+}$indicates presence of the thymidylate synthase gene enabling the organism to grow in absence of added thymidine. $\left({ }^{c}\right.$ ) Indicates chromosomally replaced gene. 


\subsubsection{Genomic plasmid DNA extraction}

Genomic DNA was isolated from L. pneumophila using the Maxicell 16 DNA purification machine with paramagnetic particles (Promega, Madison, WI). $400 \mu \mathrm{l}$ of overnight $L$. pneumophila culture was added directly to the first well, and the machine was run under the bacteria setting. Typically, $200 \mu \mathrm{l}$ of purified DNA $(\sim 50 \mathrm{ng} / \mu \mathrm{l}-130 \mathrm{ng} / \mu \mathrm{l})$ was obtained that was suitable as PCR template $(0.1 \mu \mathrm{g})$ and stored at -20 degrees.

\subsubsection{Plasmid DNA extraction}

Plasmid DNA (listed in Table 2) was isolated from E. coli and L. pneumophila using the QIAGEN Plasmid Miniprep Kit (QIAGEN Inc., Valencia, CA) following manufacturer's instructions. The only exception was L. pneumophila was incubated with lysis buffer P2 for 10 minutes on ice to increase bacterial lysis and plasmid DNA yield. For the miniprep kit, $5 \mathrm{ml} E$. coli cultures were grown overnight, harvested by centrifugation $(4000 \mathrm{x} \mathrm{g}, 6 \mathrm{~min}$, room temperature), and resuspended in $250 \mu \mathrm{l}$ buffer P1. Following addition of $250 \mu \mathrm{l}$ buffer P2, solutions were mixed by inversion until clarification. The only exception was L. pneumophila was incubated with lysis buffer P2 for 10 minutes on ice to increase bacterial lysis and plasmid DNA yield. Then, three hundred microliters of neutralization buffer N3 was added, solutions were mixed by inversion, and solutions were centrifuged at $13,000 \mathrm{rpm}$ for $10 \mathrm{~min}$ at room temperature. The resulting supernatants were added to columns and subjected to centrifugation at 13,000 rpm for $1 \mathrm{~min}$. After addition of $750 \mu \mathrm{l}$ of PE wash buffer, columns were subjected to centrifugation again and the flow-through was discarded. The columns were centrifuged once more under the same conditions to remove residual ethanol. Plasmid DNA was eluted in EB buffer in a final volume of $50 \mu 1$. 


\subsubsection{Polymerase chain reaction}

Polymerase chain reaction (PCR) amplifications were conducted with either Taq DNA polymerase (Life Technologies, Grand Isand, NY) or Expand High Fidelity Polymerase (Roche, Mannheim, Germany). All oligonucleotides (Table 3) were synthesized by Integrated DNA Technologies, Inc. (IDT) (Coralville, IA). Typically, $50 \mu 1$ reactions ( $8 \mathrm{ul} 1.25 \mathrm{mM}$ dNTPs, $3 \mu 1$ $25 \mathrm{mM} \mathrm{MgCl} 2,5 \mu \mathrm{l}$ of $10 \mathrm{x}$ reaction buffer, $400 \mathrm{ng}$ of each primer, $1 \mu \mathrm{l}$ of polymerase, and $0.1 \mu \mathrm{g}$ of template). Unless otherwise indicated, PCR conditions were as follows: an initial heating for 5 min at 94 degrees; then 35 cycles of denaturation at 94 degrees for $30 \mathrm{sec}$, annealing at 60 degrees for $30 \mathrm{sec}$, then elongation at 72 degrees ( 1 min per $\mathrm{kb}$ of amplicon). The final elongation step was 72 degrees for $7 \mathrm{~min}$, then 4 degrees indefinitely. All PCR reactions were carried out in a Gene Amp PCR system 2400 (Perkin Elmer, Norwalk, CT USA). For colony PCR, one half of a colony was harvested using a sterile toothpick, placed in $100 \mu \mathrm{l}$ of sterile $\mathrm{ddH}_{2} \mathrm{O}$, boiled for 10 min, and centrifuged at $13,000 \mathrm{rpm}$ at room temperature to remove cell debris. 1 to $2 \mu \mathrm{l}$ of this solution was used as template DNA in PCR reactions. Amplicons were stored at $4{ }^{\circ} \mathrm{C}$ for use within $24 \mathrm{~h}$, or at $-20^{\circ} \mathrm{C}$ for long-term storage.

\subsubsection{Agarose gel electrophoresis}

Electrophoresis of DNA was performed in $1 \times$ Tris acetate EDTA buffer (TAE) buffer, diluted from a $50 \mathrm{x}$ stock, per liter: $242 \mathrm{~g}$ Tris base, $57 \mathrm{ml}$ glacial acetic acid, and $100 \mathrm{ml} 0.5 \mathrm{M}$ EDTA pH 8.0, using $1 \%$ agarose gels containing 1 x TAE buffer and $1 \mu \mathrm{g} / \mathrm{ml}$ ethidium bromide. Loading buffer was added to DNA samples prior to loading into the wells of the gel. Agarose gels were subjected to electrophoresis at $125 \mathrm{~V} / \mathrm{cm}$ for desired amounts of time. For visualization 
of DNA, a UV transilluminator (Fotodyne, WI) was used and photographs were taken. DNA bands of interest were excised from gels using a scalpel blade.

\subsubsection{Purification of DNA from agarose gels}

Purification of DNA fragments from agarose gels for subcloning was carried out using a QIAquick gel purification kit (QIAGEN Inc., Valencia, CA) as described by the manufacturer using a microcentrifuge. For every $100 \mathrm{mg}$ of agarose, $300 \mu \mathrm{l}$ of QG solubilization buffer was added and agarose was placed at 50 degrees until the gel slice had completely dissolved. To ensure optimal DNA adsorption to the filter, 1 gel volume of isopropanol was added to the mixture before being loaded into the spin column. After centrifugation $(13,300 \mathrm{rpm}, 1 \mathrm{~min})$, flow through was discarded and DNA on the column was washed with $500 \mu \mathrm{l}$ of buffer QG to remove all traces of agarose and centrifuged as before. A second wash with $750 \mu$ of Buffer PE (ethanol added) was performed and centrifuged. After discarding flow through, the column was centrifuged dry to remove all traces of ethanol. DNA was eluted in $30 \mu \mathrm{l}$ of elution buffer. 
Table 3. Oligonucleotides used in this Study

\begin{tabular}{|c|c|}
\hline Primer & Sequence \\
\hline DsbA1-1F & AGTACCGCCACCTAAGTCGACCTCAAGCTATAAAGGATATTC \\
\hline DsbA1- 2R & AAACTCGAGCCCGATGTCGAGCCCTCTG \\
\hline DsbA1- 3F & TCATGGATCCGCAAGGTATTAGTAC \\
\hline DsbA1- 4R & ACAGGCTTATGTCAAGTCGACGTTGCCAAAGCAGTCATTGGC \\
\hline $\begin{array}{l}\text { c1P151T L v2 } \\
\text { (P198T- F) }\end{array}$ & CATTTGATGGGTACAACAGCCTTTATAATTGG \\
\hline $\begin{array}{l}\text { c1P151T C v2 } \\
\text { (P198T-R) }\end{array}$ & CCAATTATAAAGGCTGTTGTACCCATCAAATG \\
\hline DsbA2LDNNdel & GGAATTCCATATGGCCGCAATTCAGGAAAAT \\
\hline DsbA2expBamHI & TTAGATGGATCCTTAATTGCCAGCCGCC \\
\hline DsbA2NF & TGGCAGCTGATCAGGAAAATGCTGAACAAG \\
\hline DsbA2NR & GCATTTTCCTGATCAGCTGCCATTATTGCA \\
\hline Com1FSD & GGGAATTCTAAGGGGAATTACGTGAAATTTAC \\
\hline DsbA2 R & TAAGGATCCTTAATTGCCAGCCGCC \\
\hline DsbA2 BamHIR & TAAGGATCCTTAATTGCCAGCCGCC \\
\hline DsbB1 EcoRI F & ATCGAATTCAGCCGCAATACAATATATGTTGCCGAAATC \\
\hline DsbB1 BamHI R & ACTGGATCCCGAAAGCACGCGTTTAATGCC \\
\hline DsbB2 EcoRI F & CTATCGAATTCCAGCTTCAATTATCACAGCCATCTG \\
\hline DsbB2 BamHI R & ACTGGATCCGCCTGTAAATATCCGCTGGC \\
\hline DsbD1 Xhol F & ACTGACTCGAGACAATACCGATGACAGCGATATGG \\
\hline DsbD1 Xbal R & ACTCTCTAGAAGTGTCCGTAGTTACTTTAGTTCTCATC \\
\hline DsbD2 EcoRI F & CAGAATTCCTATGCAAGAGGTCCAGTGTAGGT \\
\hline DsbD2 BamHI R & CCGGATCCTTCAACTCAAATGGCGCGCTTGCT \\
\hline
\end{tabular}




\begin{tabular}{|c|c|}
\hline c13'BH16his & \begin{tabular}{|l} 
TAAGGATCCTTAGTGATGATGATGATGATGATTGCCAGCCGCC \\
\end{tabular} \\
\hline P150T-F & AAATTAACGCCGTAACAGCCTTTGTCGTC \\
\hline P150T- R & GACGACAAAGGCTGTTACGGCGTTAATTT \\
\hline GentaSal F Xhol & ATGCTACTCGAGGTCGACTTAGGTGGCGGTACTTG \\
\hline $\begin{array}{l}\text { Lp Com1 } \\
\text { GentaSalF }\end{array}$ & ATACTGCAGCTCGCAGATAACTTTATCATTAAT \\
\hline $\begin{array}{l}\text { Lp Com1 } \\
\text { GentaSalR }\end{array}$ & ATAAAGCTTGCCAAAGGGCTTGAGTTTCCGATTG \\
\hline DsbA EcoRI & ATAATAGAATTCGAAGATATGGAAGCCGTTGC \\
\hline DsbA BamHI & ATAATAGGATCCCTTTATAGCTTGAGTTAATTAGGCTG \\
\hline DsbA 6His & TAAGGATCCTAAATTAGTGATGATGATGATGATGGGCTGATTTTCT \\
\hline DsbA EcoRI F & GAATTCAAGGGTTATATTGAGGAATTGACTA \\
\hline DsbD2 XO do Rc & CTTATTAATCACCACTGCAACCACC \\
\hline DsbB $1 \mathrm{~kb} \mathrm{c}$ & ACTCGAGTGTGTTCAGCTTCATTCGC \\
\hline DsbB XO upR C & ACAGGCTTATGTCTATTCATAGGTTC \\
\hline DsbB XO upF & GCGAGCGGGCTCTTTTACCTG \\
\hline DsbB XO doR C & CTGTGGGGGCTATTGCGGCG \\
\hline DsbB XO doF L & AGTACCGCCACCTAGATAACCCGAGC \\
\hline DsbD1 XO do Rc & GTCCACGCCATAAGCACTGGTATC \\
\hline DsbD2 XO up F & GGAAGGAGCTGGGTTAAAGCAAC \\
\hline DsbB $1 \mathrm{~kb} \mathrm{~L}$ & AATCTAGAAGCGCAGTCAAAGCGCTC \\
\hline DsbD2 XO up Rc & ACAGGCTTATGTCTATATGACCACGC \\
\hline DsbD1 XO do F & AGTACCGCCACCTTTGAACGCCGATG \\
\hline DsbD1 XO up F & CCTTAGGGCAAACAATACCGATGAC \\
\hline DsbD2 XO do F & AGTACCGCCACCTAGCGCGCCATTTG \\
\hline
\end{tabular}




\begin{tabular}{|l|l|}
\hline DsbD1 XO up Rc & ACAGGCTTATGTCCAAGAGGTGTTGC \\
\hline DsbB XO upF & ATTACTCGAGGTTTGTATGAGACCAACGTTAGC \\
\hline DsbB XO upR & ATTAGAGCTCGCATTCAACCCCTTGATTGCAG \\
\hline DsbB XO doF & ATTAGAGCTCGCCGTTTCTGCGAATGAACAC \\
\hline DsbB XO doR & ATTAGGATCCGATACCTTCCGCCACCAC \\
\hline DsbD2 XO upF & AATTCTCGAGCGTGGAGTCAAGGAAACCAAAAC \\
\hline DsbD2 XO upR & AATTCTCGAGCATATACCCGGCAAGCATCC \\
\hline DsbD2 XO doF & AATTGAGCTCGCATGAATTTGATTCTTTGCTCTG \\
\hline DsbD2 XO doR & AATTGGATCCCCAAACTGATTCATACCTATCGC \\
\hline
\end{tabular}




\subsubsection{Purification of DNA from PCR}

Purification of DNA fragments (15 kb - $200 \mathrm{bp}$ ) was performed using QIAGEN PCR clean-up kit (QIAGEN Inc., Valencia, CA) when agarose gel separation was deemed unnecessary. Manufacturer's instructions were followed using a microcentrifuge. Five volumes of buffer QC were added to each reaction before loading onto a spin column. After centrifugation, the column was washed with $750 \mu 1$ of Buffer PE (ethanol added), and after decanting flow through a second dry spin removed all traces of ethanol. DNA fragments were eluted with $30 \mu \mathrm{l}$ of EB buffer. All DNA was stored at $-20^{\circ} \mathrm{C}$.

\subsubsection{Restriction endonuclease digestion}

Restriction digestion was performed in $25-\mu l$ reactions according to instructions provided by the manufacturer (New England Biolabs [NEB], Ipswich, MA). Unless otherwise indicated, restriction digests were incubated at 37 degrees for 60 to 90 minutes; reactions consisted of $1 \mu \mathrm{g}$ DNA, 1 x reaction buffer, $1 \mathrm{x}$ bovine serum albumin (BSA), and appropriate units (U) of restriction enzyme. Often, 5'-phosphoryl groups were removed from the vector (to minimize vector self-ligation) by addition of $0.2 \mu \mathrm{l}$ of calf intestinal phosphatase (CIP) (NEB) for the final 10 minutes of the reaction. Blunt ends were created by heat inactivation of the enzyme, followed by treatment with $1 \mu \mathrm{l}$ of T4 DNA polymerase and $1 \mu \mathrm{l}$ of $10 \mathrm{mM}$ deoxynucleoside triphosphates (dNTPs) (Life Technologies, Burlington, ON) for 30 minutes at room temperature or overnight at $16^{\circ} \mathrm{C}$. Reactions were terminated by addition of DNA loading buffer $(200 \mathrm{mM}$ Tris- $\mathrm{HCl}, \mathrm{pH} 7.5$, $200 \mu \mathrm{M}$ ethylenediaminetetraacetic acid [EDTA] $\mathrm{pH} 8.0,20 \%$ glycerol and $0.15 \%$ xylene cyanol)(Sambrook and Russel, 2001) and subjected to gel electrophoresis; alternatively, reactions 
were terminated by addition of 5 volumes of buffer QG included in the PCR Cleanup Kit supplied by QIAGEN (QIAGEN) and purified as described above (PCR purification methodology).

\subsubsection{DNA ligation}

DNA ligations were performed using T4 DNA ligase (NEB). A 1:3 to 1:5 molar ratio of vector to insert was typically used with a vector concentration of $50 \mathrm{ng}$. Reactions consisted of appropriate volumes of vector and insert DNA, 1 x final concentration of T4 DNA Ligase Buffer (NEB), and $1 \mu \mathrm{l}$ of T4 DNA ligase, with the final volume adjusted with sterile $\mathrm{ddH}_{2} \mathrm{O}$. Ligations were then left for 1.5 hours at room temperature, or overnight at $16^{\circ} \mathrm{C}$.

\subsubsection{Preparation of electrocompetent cells}

\subsubsection{E. coli}

E. coli cultures were grown overnight at $37^{\circ} \mathrm{C}$ with aeration $(200 \mathrm{rpm})$. LB broth was then inoculated 1:20 with the overnight culture, and allowed to grow for two hours until an $\mathrm{OD}_{600}$ of $\sim 0.6$ was obtained. All harvests were performed at $3000 \mathrm{x}$ g for 15 minutes at four degrees. Cells were then incubated for 5 minutes on ice. The pellet was then resuspended in $40 \mathrm{ml}$ of $0.1 \mathrm{M}$ $\mathrm{CaCl}_{2}$, followed by $20 \mathrm{~min}$ incubation on ice, and then harvested by centrifugation ( $4800 \mathrm{xg}, 6$ minutes, $\left.4^{\circ} \mathrm{C} ; 3 \mathrm{x}\right)$. The pelleted cells were then resuspended in $4 \mathrm{ml}$ of $0.1 \mathrm{M} \mathrm{CaCl}_{2}$. Cells were then aliquoted into $200 \mu \mathrm{l}$ fractions and stored at $-85^{\circ} \mathrm{C}$ until use. 


\subsubsection{L. pneumophila}

All L. pneumophila strains were grown at $37^{\circ} \mathrm{C}$ for $48 \mathrm{~h}$ on $\mathrm{BCYE}$ medium with appropriate selection conditions, and then transferred to fresh media for additional $24 \mathrm{~h}$ incubation at $37^{\circ} \mathrm{C}$ as heavy lawns on BCYE. Bacterial lawns were harvested using sterile disposable loops, and resuspended in $20 \mathrm{ml}$ of sterile $\mathrm{ddH}_{2} \mathrm{O}$ by pipette. Cells were then centrifuged (3000 x g, 10 minutes, 4 degrees) and resuspended in $20 \mathrm{ml}$ of cold $\mathrm{ddH}_{2} \mathrm{O}$, this process was repeated a total of three times. The final pellet was resuspended in $200 \mu \mathrm{l}$ of cold $15 \%$ glycerol and stored at $-85^{\circ} \mathrm{C}$ in $50 \mu$ aliquots.

\subsubsection{Electroporation of plasmids into E. coli or L. pneumophila}

Electrocompetent E. coli or L. pneumophila cells were thawed on ice and then mixed with 1-5 $\mu 1$ of plasmid DNA. The mixture was then added to a 1-mm gapped electroporation cuvette and placed on ice for $5 \mathrm{~min}$. Electroporation was performed at $1.1 \mathrm{kV}, 200 \Omega, 25 \mu \mathrm{FD}$. The cuvette was then flooded with $500 \mu 1$ of pre-warmed media (for E. coli: LB without antibiotics; for L. pneumophila: BYE without antibiotics, ferric pyrophosphate, L-cysteine plus thymidine if appropriate), the mixture removed and incubated at $37^{\circ} \mathrm{C}$ for $1-2$ hours with gentle agitation (100 $\mathrm{rpm}$ ) in a micro-centrifuge tube. One hundred microliters of the transformation is then spread on medium (LB or BCYE) or the tube was spun down at $4000 \mathrm{rpm}$ for 4 minutes, and the pellet resuspended and plated on $\mathrm{LB}$ or $\mathrm{BCYE}$ with appropriate antibiotics, and incubated at $37^{\circ} \mathrm{C}$ for 24 h (for E. coli) or 3-5 days (for L. pneumophila) for colonies to appear. Resulting colonies were replica plated onto fresh medium and incubated overnight at $37^{\circ} \mathrm{C}$. PCR analysis and DNA sequencing were used to confirm the resulting transformants. 


\subsubsection{Phylogenetic analysis}

DsbA1 and DsbA2 amino acid sequences used in phylogenetic analysis were obtained from the Legiolist web server (http://genolist.pasteur.fr/LegioList/genome.cgi) and orthologous genes from GenBank by BLASTP search using DsbA1 and DsbA2 of L. pneumophila Philadelphia-1 and DsbA, DsbC, and DsbG from E. coli. Phylogenetic trees were generated by multiple sequence alignment of unedited DsbA2 sequences using CLUSTALW. Analyses were further refined by BLASTP search using the highly conserved dimerization domain sequence (SLSDAQKKEIEKVIHDYLINNPEVLLEASQA). Protein prediction software Phyre (http://www.sbg.bio.ic.ac.uk/ phyre) was used to align DsbA2 onto the crystal structure of DsbA of E. coli.

\subsection{Protein techniques}

\subsubsection{Sodium Dodecyl Sulfate-Polyacrylamide Gel Electrophoresis (SDS-PAGE)}

SDS-PAGE analysis of proteins was performed by standard techniques described in Sambrook and Russell (2001). Briefly, protein samples were harvested as further described below in the next section or from bacterial cultures. Pellets were resuspended in SDS sample buffer ( $250 \mathrm{mM}$ Tris$\mathrm{HCl} \mathrm{pH} 6.8,20 \%$ glycerol, $2 \%$ SDS, $5 \% \beta$-mercaptoethanol, $0.001 \%$ bromophenol blue) and then boiled for $10 \mathrm{~min}$. After centrifugation to pellet cell debris, $15 \mu \mathrm{l}$ of protein lysate were resolved on Invitrogen Nu-Page Bis/Tris 4-12\% SDS-PAGE at $200 \mathrm{~V}$ for $45 \mathrm{~min}$. Gels were then transferred to coomassie blue stain, silver stain, or western blot using specific antibody to targeted protein. 


\subsubsection{Diagonal gel electrophoresis of DsbA1 cellular extracts}

Preparation of cellular extracts and diagonal gel electrophoresis were as described by Kadokura et al. (2004) and Jameson-Lee et al (2011). Briefly, 41 of AA100 strain (with DsbA1 P150T construct) was induced at an $\mathrm{OD}_{600}$ of 1.0 with $1 \mathrm{mM}$ IPTG for $15 \mathrm{~h}$. The cells were centrifuged and washed once with $1 / 10$ volume $\mathrm{ddH}_{2} \mathrm{O}$, then resuspended in $1 / 10$ volume cold $\mathrm{ddH}_{2} \mathrm{O}$. Dry TCA was added to $\sim 8 \%$, the cells were lysed on ice for $20 \mathrm{~min}$, centrifuged for 10 min, and the pellet washed twice with acetone and dried. The pellet was alkylated in $100 \mathrm{mM}$ Tris- $\mathrm{HCl}(\mathrm{pH} 8.0)$ containing $0.5 \%$ SDS and $100 \mathrm{mM}$ iodoacetamide. The alkylated lysate was then diluted four times with $50 \mathrm{mM}$ Tris- $\mathrm{HCl}(\mathrm{pH} 8.0)$ containing $300 \mathrm{mM} \mathrm{NaCl}$ and centrifuged at $10000 \mathrm{~g}$ for $20 \mathrm{~min}$ at $4^{\circ} \mathrm{C}$ to pellet insoluble material. The cleared lysate was loaded onto four separate $1 \mathrm{ml} \mathrm{Ni}^{++}$columns pre-equilibrated with buffer A [50 mM Tris- $\mathrm{HCl}(\mathrm{pH} 8.0), 300 \mathrm{mM}$ $\mathrm{NaCl}, 0.1 \% \mathrm{SDS}]$. The columns were washed with eight column volumes of buffer A containing $20 \mathrm{mM}$ imidazole and protein fractions eluted with five column volumes of buffer $\mathrm{A}+250 \mathrm{mM}$ imidazole. Fractions containing DsbA1 P150T-His 6 were pooled and concentrated by spin column and centrifugation. Proteins were resolved on Invitrogen Nu-Page Bis/Tris 4-12\% SDS-PAGE at $200 \mathrm{~V}$ for $45 \mathrm{~min}$ under non-reducing conditions. The lane was excised and incubated in warm $100 \mathrm{mM}$ DTT/BME for $15 \mathrm{~min}$, then placed in Laemmli loading buffer without bromophenol blue $+100 \mathrm{mM}$ iodoacetamide for $5 \mathrm{~min}$. The excised lane was placed on top of another Invitrogen $\mathrm{Nu}-$ Page SDS Bis/Tris 4-12\% gel that had been modified by removal of gel material separating lanes as previously described (Hoffman et al., 1992). The gel slice was locked in place by addition of stacking gel, and electrophoresed at $200 \mathrm{~V}$ for desired amounts of time, followed by silver staining. 


\subsubsection{Western blot}

Proteins were transferred onto nitrocellulose membranes with the IBlot Western blotting equipment from Invitrogen for $7 \mathrm{~min}$ at $20 \mathrm{~V}$. The immune detection reaction was performed following these steps: blots were incubated $60 \mathrm{~min}$ in a $1 \mathrm{x}$ PBS blocking solution $(140 \mathrm{mM} \mathrm{NaCl}$,

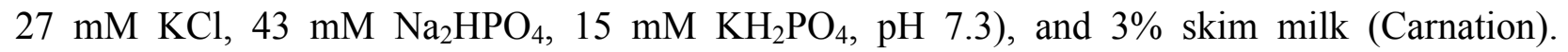
Membranes were then incubated overnight or for $60 \mathrm{~min}$ at room temperature in a $1 \mathrm{x}$ PBS supplemented with primary antiserum-antibody. Following a quick wash in $1 \mathrm{x}$ PBS, blots were incubated for $60 \mathrm{~min}$ in $15 \mathrm{ml}$ of 1x PBS with secondary antibody, membranes were washed with 1x PBS for $15 \mathrm{~min}$ and then developed.

\subsubsection{Isolation of native DsbA2 by osmotic shock}

Stationary-phase bacteria (strain AA100 or expressed in E. coli) from overnight culture were collected by centrifugation at $13,000 \mathrm{xg}$ for $15 \mathrm{~min}$ at $4^{\circ} \mathrm{C}$, and the pellet $(1 \mathrm{~g}$ [wet weight]) was suspended in $40 \mathrm{ml}$ of sucrose buffer $(0.5 \mathrm{M}$ sucrose, $30 \mathrm{mM}$ Tris- $\mathrm{HCl}$ plus $1 \mathrm{mM}$ EDTA at $\mathrm{pH}$ 8) and gently shaken for $10 \mathrm{~min}$ at $25^{\circ} \mathrm{C}$. Following centrifugation, the pellet was suspended in $20 \mathrm{ml}$ of ice-cold $30 \mathrm{mM}$ Tris- $\mathrm{HCl}$ plus $1 \mathrm{mM}$ EDTA buffer, gently shaken for $10 \mathrm{~min}$ at $4^{\circ} \mathrm{C}$, and centrifuged. The shockate was decanted and concentrated to $5 \mathrm{ml}$ and loaded onto the calibrated HiLoad 16/60 Superdex-200 column, and fractions corresponding to the homodimer and monomer (by molecular masses) were subjected to SDS-PAGE. The HiLoad 16/60 Superdex200 column was calibrated with RNase A (13.7 kDa), chymotrypsinogen A (25 kDa), ovalbumin (43 kDa), albumin (67 kDa), and blue dextran 2000 (void volume). The proteins were transferred to nitrocellulose and developed with DsbA2-specific antibody diluted 1:10,000 in phosphate- 
buffered saline (PBS) with $0.1 \%$ Tween 20 as previously described (Helsel et al., 1987; JamesonLee et al., 2011).

\subsubsection{Periplasmic redox status of DsbA2}

The in vivo redox status of DsbA2 was determined by alkylation of free thiol groups by 4acetamido-4'- maleimidylstilbene-2,2'-disulfonic acid (AMS) (Molecular Probes, Eugene, OR) essentially as described previously (Kadokura et al., 2004; Jameson-Lee et al., 2011). Briefly, $L$. pneumophila was grown to stationary phase $(\sim 24 \mathrm{~h})$, and half of the culture was collected by centrifugation and washed once in sterile water and then suspended to an $\mathrm{OD}_{660}$ of 0.5 in sterile filtered tap water. The water- suspended bacteria were kept in the dark for $48 \mathrm{~h}$ at $22^{\circ} \mathrm{C}$, a period of time previously shown to promote differentiation into cyst-like dormant forms (Garduno et al., 2002). The stationary-phase bacteria were collected by centrifugation and were divided into aliquots, one of which was first treated with $10 \mathrm{mM}$ DTT and then trichloroacetic acid (TCA) precipitated and alkylated with $100 \mathrm{mM}$ AMS; one was TCA precipitated and alkylated with AMS, and one served as an untreated control. The water-treated bacteria were TCA precipitated and treated with $100 \mathrm{mM}$ AMS. MalPEG 5000 ( $N$-ethylmaleimide covalently bound to polyethylene glycol) was used to alkylate free thiols of DsbA2 and DsbA2N expressed in E. coli. Aliquots were treated as described for AMS except that $2 \mathrm{mM}$ DTT was used to reduce DsbA2 prior to alkylation with $5 \mathrm{mM}$ MalPEG 5000. In both treatments, samples were analyzed by SDSPAGE and immunoblotting with anti-DsbA2 serum (Jameson-Lee et al., 2011). AMS increases protein mass by $490 \mathrm{Da}$ and MalPEG by $5,000 \mathrm{Da}$, observed as band shifts. 


\subsection{Functional and enzymatic assays}

\subsubsection{Motility assays}

E. coli wild type (JCB570), $d s b A$ mutant (JCB571), $d s b B$ mutant (RGP405), $d s b A d s b B$ mutant (RGP438) strains were kindly provided by Dr. JC Bardwell (University of Michigan, Ann Harbor, MI). Plasmid pBC or pMMB206 containing either the coding sequence of LpndsbAl, LpndsbA2, LpndsbB1, LpndsbB2, or empty vector was heat-shocked into JCB571, RGP405, or RGP438. JCB570, JCB571, RGP405, and RGP438 (with different plasmids) were grown overnight in LB and appropriate antibiotics, diluted 1:20, and grown for 2-3 hours to an $\mathrm{OD}_{600}$ of 0.7 with $1 \mathrm{mM}$ IPTG induction. Soft agar LB plates $(0.4 \%$ agar $)$ supplemented with $1 \mathrm{mM}$ IPTG were prepared, and $2 \mu \mathrm{l}$ of cell suspension was inserted into the middle of the plate. The LB plates were incubated overnight at $30^{\circ} \mathrm{C}$, and motility was assessed by measuring the diameter of spreading bacterial growth and reported as a percentage of the WT control.

\subsubsection{Insulin reduction assay}

Reductase activity was assessed by an insulin precipitation assay with minor modifications (Bardwell et al., 1991; Jameson-Lee et al., 2011). Bovine insulin was dissolved in Tris-HCl to 10 $\mathrm{mg} / \mathrm{ml}(1.67 \mathrm{mM})$ and titrated to $\mathrm{pH} 7.5$, creating a clear solution. Reactions (triplicate) were carried out in $200 \mu \mathrm{l}$ of $100 \mathrm{mM}$ sodium phosphate buffer, $\mathrm{pH} 7.0,150 \mu \mathrm{M}$ insulin, $0.33 \mathrm{mM}$ dithiothreitol (DTT), and $2 \mathrm{mM}$ EDTA; reaction mixtures were incubated in a 96-well plate format at room temperature in a VersaMax (Molecular Devices) plate reader, and absorbance was measured at $650 \mathrm{~nm}$. The insulin reduction assay was initiated by adding $5 \mu \mathrm{M}$ DsbA2, $\operatorname{DsbA} 2 \Delta \mathrm{N}$, and DsbA1 protein purified as $\mathrm{His}_{6}$-tagged proteins following induction in E. coli 
strain BL21. Both time to start of insulin reduction and specific activity were determined in triplicate using the enzyme kinetics program as previously described (Jameson-Lee et al., 2011).

\subsubsection{PDI detector assay}

The PDI detector assay utilizes the TEM1 $\beta$-lactamase of pBR322 with an engineered nonconsecutive disulfide bond (cysteine residues added, S81C and T108C) that requires disulfide bond isomerase activity to be properly folded in the E. coli periplasm (Ren \& Bardwell, 2011). Strains kindly provided by J. C. Bardwell and listed in Table 2 include RGP209 (dsbC mutant control), RGP663 plus pPDI detector plasmid (wild-type positive control), RGP665 (RGP209 plus pPDI detector plasmid), RGP666 ( $d s b D$ mutant plus pPDI detector plasmid), and RGP810 ( $d s b C d s b D$ mutant plus pPDI detector plasmid). Ampicillin resistance was evaluated at $0,1,2$, or $3 \mathrm{~g} /$ liter. For complementation studies, $d s b A 1, d s b A 2, d s b A 2 L D N, d s b A 2 \Delta N, d s b D 1$, and $d s b D 2$ were cloned into $\mathrm{pBC}$ or $\mathrm{pMMB} 206$ with selection for chloramphenicol. Bacterial cells were grown overnight in the appropriate antibiotic, diluted 1:20, and grown for 2 to $3 \mathrm{~h}$ to an $\mathrm{OD}_{600}$ of 0.7 with $1 \mathrm{mM}$ IPTG induction. Cells were then serially diluted and plated in triplicate on LB plates with 0, 1, 2, or $3 \mathrm{~g} /$ liter Amp, and CFU counts were recorded at $24 \mathrm{~h}$.

\subsubsection{Isomerization assay}

TEM1 $\beta$-lactamase (WT and PDI detector mutant enzymes Bla and MBla) was obtained from spent culture supernatants of strains RGP663 and RGP665 following overnight growth in LB medium. Supernatants were concentrated by spin column (12 $\mathrm{ml}$ concentrated to $0.5 \mathrm{ml})$ (Amicon Ultracel 10k). Bla activity was tested by spotting $5 \mu$ l of concentrated supernatant onto 
nitrocefin-impregnated paper disks (Becton, Dickinson). The relative concentration of Bla in each fraction was determined by SDS-PAGE following staining with Coomassie brilliant blue. For the PDI assay, either purified leaderless $\mathrm{H}_{6}$-tagged DsbA1 $(6.9 \mathrm{mg} / \mathrm{ml})$ or $\mathrm{H}_{6}$-DsbA2 $(3 \mathrm{mg} / \mathrm{ml})$ was added to concentrated culture supernatant from strain RGP665 in a ratio of 1:5 and incubated at $37^{\circ} \mathrm{C}$ for $30 \mathrm{~min}$. Nitrocefin (Calbiochem, La Jolla, CA) was prepared according to manufacturer's instructions for a spectrophotometric assay, and a working dilution of $500 \mu \mathrm{g} / \mathrm{ml}$ was prepared in $0.1 \mathrm{M}$ sodium phosphate buffer at $\mathrm{pH}$ 7.0. The refolding assay was configured in a 96-well microplate (Costar, Corning, NY) assay (100 $\mu 1 /$ well) containing sodium phosphate buffer and $15 \mu \mathrm{l}$ of MBla plus DsbA1 or MBla plus DsbA2, and the assay was started by the addition of $10 \mu \mathrm{l}$ of nitrocefin stock. Controls contained no DsbA2 or DsbA1 protein or contained WT Bla as a positive control. The rates of hydrolysis of nitrocefin at $486 \mathrm{~nm}$ were obtained over the linear range of the reaction $(30 \mathrm{~min})$ at $30^{\circ} \mathrm{C}$ in a Molecular Dynamics plate reader; all reactions were run in triplicate.

\subsubsection{Erythrocytes contact lysis}

Human red blood cells (UVA Blood Bank) were diluted in PBS, washed by centrifugation until supernatant was colorless, and incubated with bacterial strains at an MOI of 25:1 to 50:1 in a final volume of $\sim 1 \mathrm{ml}$ as described (Kirby et al., 1998; Charpentier et al., 2009; Jameson-Lee et al., 2011). L. pneumophila strains and red blood cells were mixed, pelleted for $3 \mathrm{~min}$ at $10000 \mathrm{~g}$, and after 1 to $2 \mathrm{~h}$ at $37^{\circ} \mathrm{C}$ followed by gentle vortexting, $100 \mathrm{ml}$ aliquots of supernatants were transferred to microtitre plates, and absorbance measured at $\mathrm{OD}_{415}$ for hemoglobin release. 


\subsubsection{HeLa cell infection}

HeLa cells were grown in Dulbecco's minimal essential medium (DMEM) with newborn calf serum (NCS) as described previously (Garduño et al., 1998c; Jameson-Lee et al., 2011). HeLa cells were resuspended in DMEM without antibiotics to a concentration of $10^{6}$ cells per ml and left to adhere for 1-2 h. L. pneumophila was standardized to an $\mathrm{OD}_{600}$ of 1.0. One hundred microlitres of the bacterial suspension was added to triplicate wells to a final inoculum of $10^{8}$ bacteria per $10^{6} \mathrm{HeLa}$ cells. Plates were centrifuged at $500 \mathrm{rcf}$ for $5 \mathrm{~min}$ at room temperature in a clinical centrifuge to maximize contact of bacteria with the HeLa cell monolayer and then incubated for $3 \mathrm{~h}$ to facilitate infection. Following incubation, monolayers were washed six times with PBS, lysed with water and vigorous pipetting and the remaining bacteria (representing $L$. pneumophila which as attached or invaded the HeLa cells), was then serially diluted and plated in triplicate on BCYE agar for bacterial enumeration.

\subsubsection{A. castellanii infection}

Acanthamoeba castellanii (ATCC 30010) was maintained in ATCC medium 712 at $25^{\circ} \mathrm{C}$. For infection, a $48 \mathrm{~h}$ culture of $A$. castellanii was washed and resuspended in Tris-buffered salt solution containing $2 \mathrm{mM} \mathrm{KCl}, 1 \mathrm{mM} \mathrm{CaCl}, 0.5 \mathrm{mM} \mathrm{MgCl}_{2}$ and $1 \mathrm{mM}$ Tris, with the $\mathrm{pH}$ adjusted to 6.8-7.2 (Berk et al., 1998). Approximately $10^{5}$ A. castellanii per ml were infected with $10^{3}$ cfu per ml of L. pneumophila from an overnight culture grown in the presence or absence of IPTG, as previously described (Morash et al., 2009; Jameson-Lee et al., 2011). All experiments were performed in triplicate, incubated at $30^{\circ} \mathrm{C}$, and at each time point wells were scraped to remove adherent amoeba. Bacteria were enumerated via decimal dilution in sterile 
water and plating on BCYE agar plates as previously described (Morash et al., 2009). Data are presented as the mean and standard deviation.

\subsubsection{L929 infectivity assay}

Murine L929 cells were grown and maintained in $1 \times$ DMEM supplemented with $10 \%$ bovine serum. For plaque assay, $\sim 5 \times 10^{5}$ cells were added to each well of a 24 -well tissue culture plate. After allowing the cells to adhere for $2-3 \mathrm{~h}$, the confluent monolayers were washed once with PBS, and received $200 \mu 1$ of 1 x DMEM (with $10 \%$ bovine serum). Stationary phase grown L. pneumophila cell were harvested and standardized to an $\mathrm{OD}_{600}$ of $0.5 \mathrm{in} 1 \mathrm{ml}$ of $1 \mathrm{x}$ DMEM (with $10 \%$ bovine serum). Cells were then serially diluted 10 -fold in $900 \mu \mathrm{l}$ of $1 \mathrm{x}$ DMEM (with 10\% bovine serum). Immediately after removing the culture medium from the L929 cells, $400 \mu \mathrm{l}$ of each dilution was added to duplicate wells. Plates were then subjected to centrifugation $(1,000 \times \mathrm{g}, 10 \mathrm{~min})$ at room temperature and incubated for $3 \mathrm{~h}$ at $37^{\circ} \mathrm{C}$. Following incubation, monolayers were washed six times with $1 \mathrm{ml}$ DMEM before the addition of $1 \mathrm{ml}$ of $0.6 \%$ agarose (SeaKem) in $1 \mathrm{x}$ DMEM (with bovine serum) supplemented with appropriate antibiotic for selection. Cells were then incubated at $37^{\circ} \mathrm{C}$ for four days. After four days, $1 \mathrm{ml}$ of PBS containing $10 \%$ formalin was added to each monolayer (gently placed under agarose plugs), and then incubated for $2 \mathrm{~h}$ to $24 \mathrm{~h}$ at room temperature. The agarose plugs were gently removed and the PBS/formalin solution was washed off the monoloyer with $\mathrm{ddH}_{2} \mathrm{O}$, and the monolayer were stained for $1 \mathrm{~h}$ with $1 \%$ crystal violet in $20 \% \mathrm{EtOH}$. Excess stain was then removed with $\mathrm{ddH}_{2} \mathrm{O}$ and plates were left to air dry as described (LeBlanc, Dalhousie University thesis, 2006). Plaques in the monolayer were counted and plaquing efficiency was calculated as (number of plaques formed/ viable count of the inoculum) x 100 . 


\subsection{Other methods}

\subsubsection{Complementation studies}

Wild-type (WT) E. coli (JCB570) and a $d s b A$ mutant (JCB571), $d s b B$ mutant (RGP405), and a $d s b A d s b B$ double mutant (RGP438) were kindly provided by J. C. Bardwell. The plasmid $\mathrm{pBC}$ or pMMB206 containing the coding sequence of $d s b A, d s b A 2$, or $d s b A 2 \Delta N$ were transformed into JCB571, and $\mathrm{p} d s b A 1$ (or with either $\mathrm{p} d s b B 1$ or $\mathrm{p} d s b B 2$ ), $\mathrm{p} d s b A 2$ (or with either $\mathrm{p} d s b B 1$ or $\mathrm{p} d s b B 2$ ), $\mathrm{p} d s b A 2 \Delta N$ (or with either $\mathrm{p} d s b B 1$ or $\mathrm{p} d s b B 2$ ) were transformed into RGP438. Also, pdsbB1, pdsbB2, or empty vector was transformed into the (RGP405) E. coli strain. Appropriate antibiotic(s) resistance was used (see Table 2) for selection of all constructs.

\subsubsection{Bioinformatics and analyses}

Phylogenetic analyses of the various Dsb proteins of L. pneumophila (DsbB1, DsbB2, DsbD1 and DsbD2) was performed using CLUSTALW. Transmembrane topology of DsbB and DsbD proteins was determined using TMHMM Server 2 software and amino acid positioning by alignments. BLASTP was used to evaluate relatedness of DsbB and DsbD proteins.

\subsubsection{Phylogenetic analysis of DsbA1, DsbA2 and EcDsbC}

DsbA1 and DsbA2 amino acid sequences used in phylogenetic analysis were obtained from the LegioList web server (http://genolist.pasteur.fr/LegioList/genome.cgi) and orthologous genes from GenBank by BLASTP search using DsbA1 and DsbA2 of L. pneumophila Philadelphia-1 and DsbA, DsbC, and DsbG from E. coli. Phylogenetic trees were generated by multiple sequence alignment of unedited DsbA2 sequences using CLUSTALW. Analyses were 
further refined by BLASTP search using the highly conserved dimerization domain sequence (SLSDAQKKEIEKVIHDYLINNPEVLLEASQA).

\subsubsection{Construction of DsbA2 $\Delta N$}

To construct an in-frame deletion of the dimerization domain of DsbA2 (DsbA2 $\Delta \mathrm{N})$, a vector-free strategy was employed using overlapping primers (DsbA2NF \& DsbA2NR) to join the 5 ' leader sequence with sequences downstream of the dimerization domain, and using flanking primers Com1FSD and DsbA2BamHIR to amplify the joined construct. The resulting amplicon was cloned into $\mathrm{pBC}$ and $\mathrm{pMMB} 206$ vectors.

\subsubsection{Construction of ZK01 and ZK02}

Utilizing RGP438 ( $\Delta d s b A d s b B)$ mutant strain, we replaced the chromosomal copy of the E. coli $d s b D$ with either LpnDsbD1 or LpnDsbD2 resulting to genetically altered strains ZK01 and ZK02, respectively. We utilized a vector free strategy using primers (DsbD1 XhoI F \& DsbD1 Xbal R) and (DsbD2 EcoRI F \& DsbD2 BamHI R) to amplify $d s b D 1$ and $d s b D 2$, we then amplified a gentamicin cassessette using primer pairs (GentaSal F XhoI \& Lp Coml GentaSaIR) and (Lp Com1 GentaSaIF \& Lp Com1 GentaSaIR). The amplicons were digested with appropriate enzymes and ligated creating $d s b D 1$-gent and $d s b D 2$-gent. The constucts were electroporated into RGP438 competent cells and plated on antibiotic plates for selection of chromosomally replaced $d s b D$. Colonies of the constructs were checked by PCR with primers (DsbD1 XhoI F \& Lp Com1 GentaSaIR) and (DsbD2 EcoRI F \& Lp Com1 GentaSaIR); PCR products were validated by visualization of gel electropheresis. Following validation, DsbA2 with 
either DsbB1 or DsbB2 in pMMB206 inducible vector (pA2B1 or pA2B2) were added to ZK01 or ZK02, and then utilized in motility assay as described above (section 2.4.1) to assess DsbA2 oxidase activity. 


\section{Chapter 3. Results}

\subsection{Phylogenetic analysis}

Previous phylogenetic analysis of the DsbA2 clade indicated that this group had diverged from the DsbA lineage of disulfide bond oxidoreductase (Jameson-Lee et al., 2011). Comparison of the structures of DsbA2, DsbA, and DsbC shows that DsbA2 contains an additional 56 amino acids ( 86 total) from the N-terminus to the CXXC motif compared with 31 for DsbA and 107 for DsbC. A refined BLASTP search using the dimerization domain region depicted in Fig. 6A revealed a 27-amino-acid (aa) sequence (bold in Fig. 6A) present in all the bacterial species expressing DsbA2 listed in Table 1. Moreover, this sequence was more discriminating in searches than the whole protein or the C-terminal DsbA-related sequences used previously (Jameson-Lee et al., 2011). Table 1 includes representative species based on a similarity cutoff of $\sim e^{-34}$ and is not meant to be comprehensive. We noticed that below this cutoff, the DsbA2 clade begins to merge into the DsbA clade. The DsbA2 clade includes a group of intracellular parasites that commonly cause disease in humans, animals, and plants and share a T4SS. Other genera in this clade that do not express T4SSs include the nitrogen-fixing endosymbionts associated with leguminous plants, including Bradyrhizobium and related genera (not listed), and many soil and aquatic free-living genera, including Azospirillum Rhodopseudomonas, Rhodospirillum, and Caulobacter. One exception is Bordetella pertussis, which has a T4SS (pertussis toxin [PtL] transporter) and expresses a DsbA/DsbC system similar to the $E$. coli system.

In addition, the spacing between the CXXC motif and the resolving cis-proline motif is conserved between DsbA2 and DsbA compared with DsbC. Finally, as highlighted in Fig. 5B, the first amino acid at the $\mathrm{N}$-terminus of the cis-proline in DsbA2 (threonine) is similar to that of 


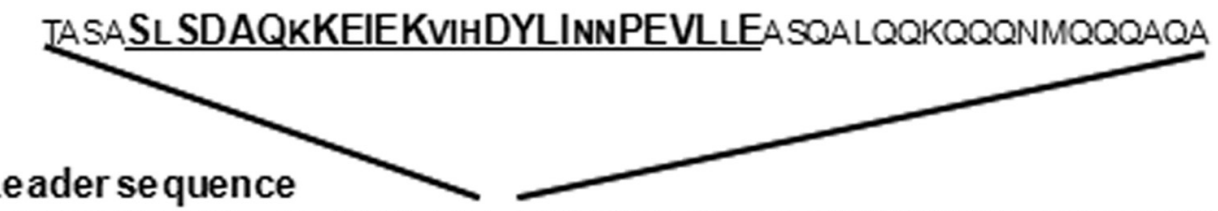

MKFTSLLTAGALASTLVSPAIMEAAAD QENAEQVFQGKLTTVGNPKGNVTLVEFFDYQCIHCKKMASTIENL VKKDSGLRVIYKEFFIFGKTSDLASRVALAAGMQGKYQAMHNALIIDKRLDEKTVMDAAKSIGLDMQKLK KDMDSQEVTDILDANRQLAEKLHLMGTPAFIIGSTPDGQYKKGSEISFIPGATSEQSLRELIKKAAGN

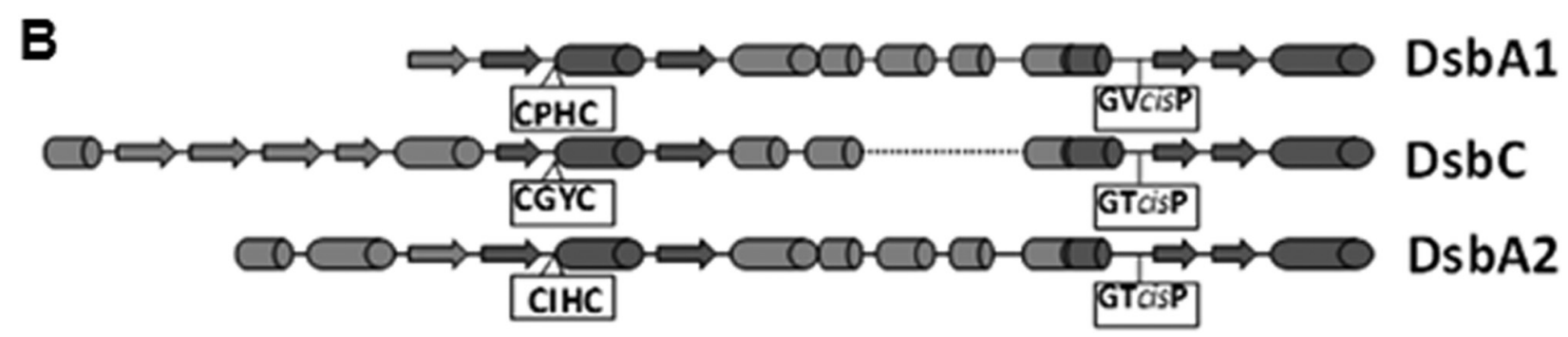

Figure 6. Dimerization domain and secondary structure comparison among DsbA proteins of Lpn and DsbC of E. coli. (A) DsbA2 amino acid sequence depicting the leader sequence and the detached dimerization domain sequence deleted to create DsbA2 $\Delta \mathrm{N}$. The underlined sequences (27 amino acids) of the dimerization domain are conserved, and larger letters represent amino acids that are highly conserved among DsbA2 members listed in Table 1. The CXXC and cis-proline regions are in boldface. (B) Secondary structure predictions for $L$. pneumophila DsbA1, which is similar to DsbA of E. coli, E. coli DsbC, and L. pneumophila DsbA2 are depicted. The CXXC region and the cis-proline motifs are boxed. Note that the cis- $P$ region of $\mathrm{DsbA2}$ is similar to that of $\mathrm{DsbC}$. 
DsbC and not of DsbA which substitutes valine. Further genomic analysis using E. coli DsbC and DsbG amino acid sequences in the BLASTP searches revealed that the genera expressing DsbA2 listed in Table 1 also lacked orthologues of DsbC and DsbG. Our previous studies showed that $L$. pneumophila $\triangle d s b A 1$ was indistinguishable from the wild-type strain for motility and infectivity for amoebae and HeLa cells, and in the close relative Coxiella burnetii, dsbA1 is completely absent from sequenced genomes (Jameson-Lee et al., 2011). While there is plenty of precedent for interchangeability of DsbA and DsbC functions in E. coli (Bader et al., 2001; Segatori et al., 2004), we investigated whether DsbA2 represents a functional equivalent of DsbC or if equilibrium of monomers and homodimers is responsible for the apparent bifunctional phenotype and thus a variation of the $E$. coli $\mathrm{DsbA} / \mathrm{DsbC}$ paradigm.

\subsection{DsbA2 exists as a homodimer in $E$. coli and $L$. pneumophila}

To test whether DsbA2 exists as a mixture of monomers and dimers, size exclusion chromatography was employed. For this study, His 6 -tagged DsbA1 and DsbA2 were treated with iodoacetamide to limit formation of spurious disulfides prior to nickel interaction chromatography. Each purified protein was subsequently applied to a calibrated gel filtration column, and, as seen in Fig. 7A, DsbA1 eluted at 95 min, with an apparent mass of $27 \mathrm{kDa}$, consistent with a monomer. In Fig. 7B, DsbA2 eluted as a single peak at 75 min, with an apparent mass of $60 \mathrm{kDa}$, consistent with the size of the homodimer. Subsequent purifications of DsbA2 indicated that inclusion of iodoacetamide was unnecessary.

To ensure that the homodimeric form was not an artifact generated in E. coli, we applied osmotic shockates from L. pneumophila similarly treated with iodoacetamide over the same calibrated column, and DsbA2 eluted as the dimer (Fig. 7C). 


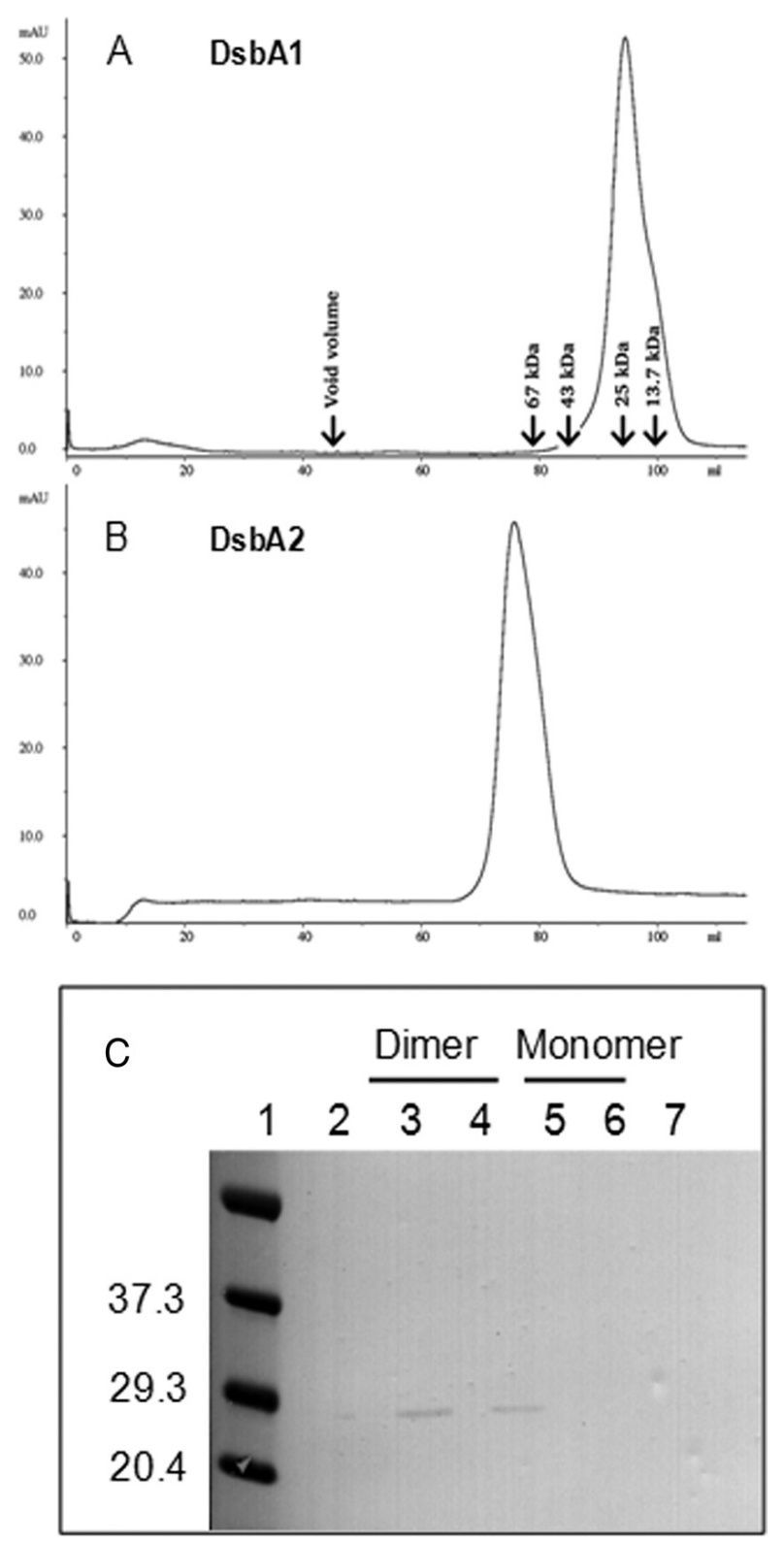

Figure 7. Size exclusion of native proteins from L. pneumophila.

(A) DsbA1 elutes as a single peak at 95 min, consistent with a monomer and an estimated mass of $25 \mathrm{kDa}$. (B) DsbA2 elutes at $75 \mathrm{~min}$, consistent with the homodimer and estimated mass of $60 \mathrm{kDa}$. (C) Immunoblot of DsbA2 containing fractions prepared by osmotic shock from L. pneumophila and collected from the calibrated column. The DsbA2 protein from $L$. pneumophila eluted at $75 \mathrm{~min}$, consistent with the homodimer. The immunoblot was developed with antibody specific for DsbA2. 
The elution of DsbA2 as a single peak suggests that if the monomer form is present in $L$. pneumophila, it is below the limit of detection in our assay.

\subsection{Lpn $d s b A 1$ and $d s b A 2 \Delta \mathrm{N}$, but not $d s b A 2$ restore motility to Ec $d s b A$ mutant}

In E. coli, DsbA is required for disulfide bond formation in the flagellar P-ring protein (FlgI), which is part of the flagella motor structure that is necessary for flagella driven motility in soft agar (Dailey \& Berg, 1993). As seen in Fig. 9, wild-type E. coli strain JCB570 is motile in $0.4 \%$ soft agar, whereas $\Delta d s b A$ mutant strain JCB571 is not. We introduced Lpn DsbA1 and DsbA2 expressed from pBC plasmids into JCB571 to test whether expression of these proteins could restore motility. As seen in Fig. 9, $d s b A 1$ complemented motility to $\sim 60 \%$ that of wild type (diameter of spreading growth), whereas $d s b A 2$ did not, consistent with the general view that dimeric proteins, such as DsbC, are poor substrates of the E. coli DsbB (Bader et al., 2001; Segatori et al., 2004; Shouldice et al., 2011). We next removed the dimerization domain of DsbA2 (DsbA2 $\Delta \mathrm{N})$ by an in-frame deletion that retained the signal sequence as depicted in Fig. $6 \mathrm{~A}$ to create the monomer. The DsbA2 $2 \mathrm{~N}$ monomer was confirmed by gel filtration (Fig. 8). Expression of $d s b A 2 \Delta \mathrm{N}$ in JCB571 restored motility to nearly the wild-type level ( $70 \%)$ of the control (Fig. 9). While growth rate differences might account for the partial complementation in our studies, these were not apparent. It is more likely that these differences reflect different efficiencies of DsbA1 and DsbA2 $\Delta \mathrm{N}$ oxidation by E. coli DsbB compared with DsbA. These results indicate that it is the dimerization domain of DsbA2 that prevents interaction of the homodimer with DsbB. 


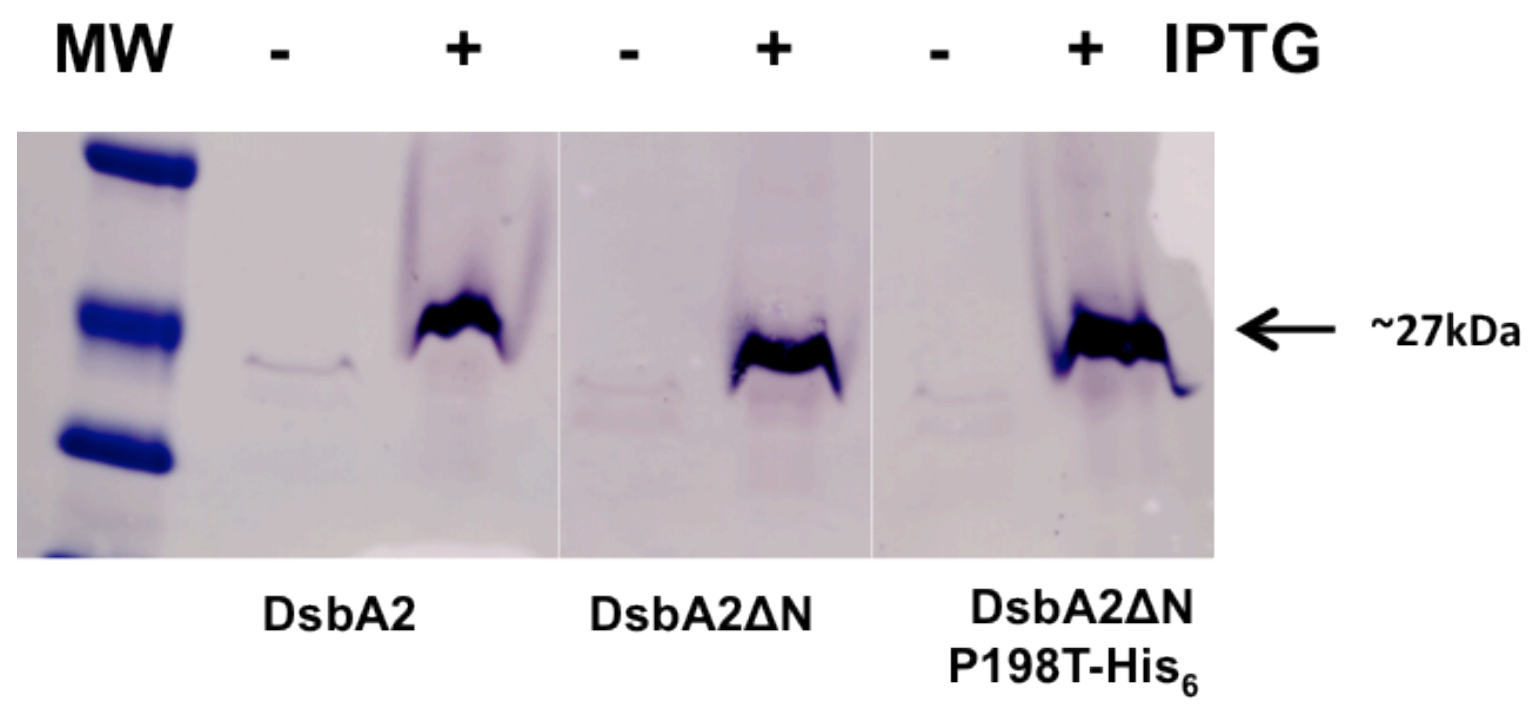

Figure 8. Western blot of Dsb2 $\Delta \mathrm{N}$ mutant constructs. Protein expression of all constructs using $1 \mathrm{mM}$ IPTG at stationary phase. The immunoblot was developed with antibody specific for DsbA2. 


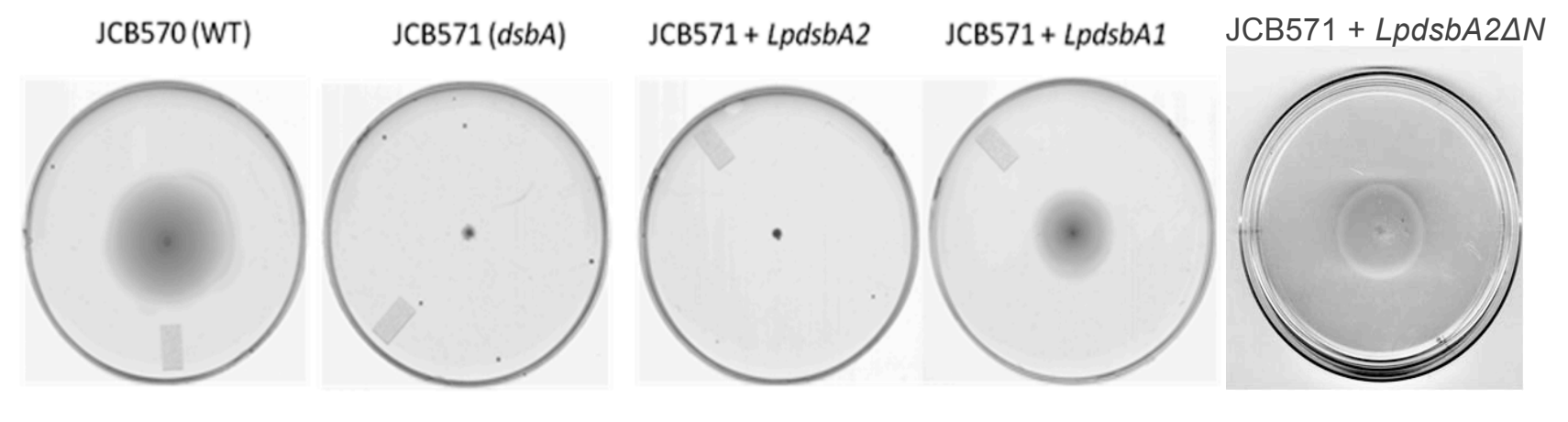

Figure 9. Complementation of motility defect in soft agar. Spreading motility by $E$. coli in soft agar is dependent on functional flagella and chemotaxis. (A) Wild-type E. coli (JCB570) is motile in soft agar at $30^{\circ} \mathrm{C}$. Strain JCB571 $(d s b A)$ has a $d s b A$ mutation and is nonmotile in this medium. Strain JCB571(pBCLpndsbA2) did not complement the motility defect, whereas JCB571(pBCLpndsbA1) complemented $(\sim 60 \%)$ this strain for motility. Also, expression of JCB571 (pBCLpndsbA2 $\Delta N$ ), which contains an in-frame deletion of the dimerization domain depicted in Fig. 6A, restored motility to $\sim 70 \%$ of wild-type strain JCB570 activity. The results presented in both panels are representative of typical results obtained from triplicate platings. $\mathrm{Lp}=\mathrm{Lpn}$ in data. 


\subsection{Periplasmic redox status of monomeric and homodimer DsbA2 in E. coli}

In Gram-negative bacteria, DsbA is maintained fully oxidized by DsbB, while DsbC is maintained fully reduced by the DsbD system. We previously reported that DsbA2 existed as a mixture of oxidized and reduced forms in L. pneumophila during stationary phase (Jameson-Lee, UVa thesis, 2012). To evaluate the redox status of DsbA2 and DsbA2 $\Delta \mathrm{N}$ in E. coli, we used the thiol alkylating agent MalPEG5000 to alkylate free thiols, and those thiols reactive with MalPEG resulted in an increased mass of 5,000 Da per cysteine residue. As seen in Fig. 10, whole E. coli bacteria expressing DsbA2 were first treated with DTT before alkylation with MalPEG (lane 1) or in the absence of prior DTT treatment (lane 3) and in both cases showed shifted high-molecularweight bands resulting from complete alkylation by MalPEG relative to untreated control (lane 2). In contrast, when DsbA2 $\Delta \mathrm{N}$ was treated with reducing agent and then MalPEG, multiple shifted bands were observed (Fig. 10, lane 6), whereas treatment with MalPEG without reducing agent (lane 8) produced no shifted bands and showed no differences from that of the untreated control (lane 7), indicating that $\operatorname{DsbA} 2 \Delta \mathrm{N}$ is maintained as the disulfide in E. coli.

These results indicate that $\mathrm{DsbA} 2$ but not $\mathrm{DsbA} 2 \Delta \mathrm{N}$ is fully reduced to the free thiol in the periplasm of E. coli.

\subsection{Insulin reduction assay}

An insulin reduction assay is often used to demonstrate thioredoxin-like activity of DSBs (Holmgren, 1979; Bardwell et al., 1991). This assay can distinguish between strong oxidases like DsbA and reductases such as thioredoxin by both the time to reduction and the rate of insulin precipitation resulting from the reduction of its disulfide bond (Bessette et al., 1999). In this regard, we previously demonstrated that DsbA2 was similar to thioredoxin in this assay 

A. DsbA2
B. DsbA2 $\Delta N$

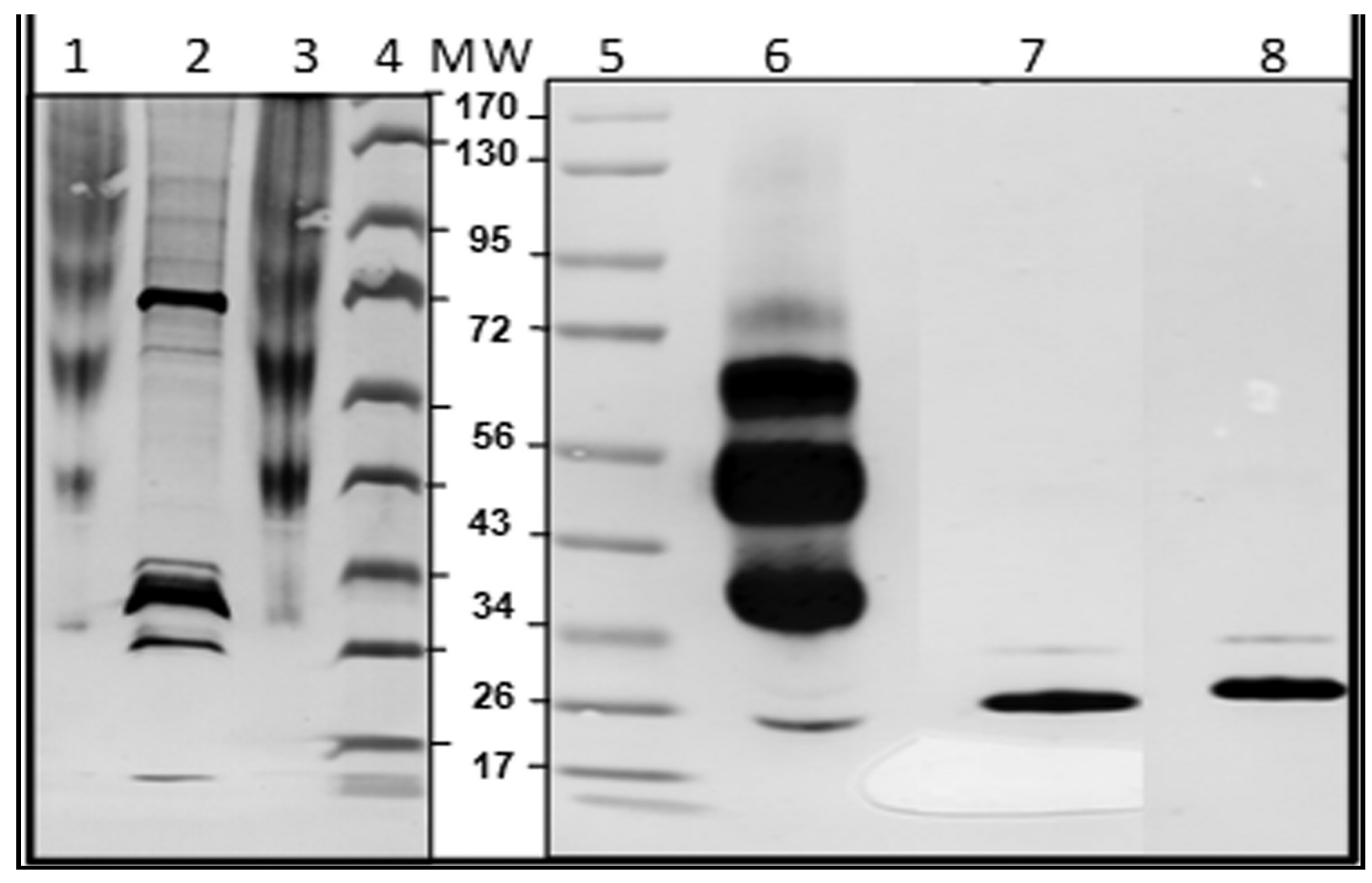

Figure 10. DsbA2 and DsbA2 $\Delta$ N redox status in E.coli. (A) Redox status of DsbA2 in E. coli. Lane 1, whole cells reduced with DTT for $30 \mathrm{~min}$ and alkylated with MalPEG5000 (reduced control); lane 2, untreated control; lane 3, whole cells treated with MalPEG5000. Lanes 4 and 5 contain the molecular weight standards. (B) Redox status of DsbA2 $\Delta \mathrm{N}$ in E. coli. Lane 6, whole cells treated with DTT for $30 \mathrm{~min}$ and then alkylated with MalPEG5000; lane 7, untreated control; lane 8, whole cells treated with MalPEG5000. The molecular weight of DsbA2 is $\sim 28,000$, and that of DsbA2 $\Delta N$ is $\sim 26,000$. Anti-DsbA2 mouse serum was used to identify the DsbA2 proteins. The multiply shifted bands are indicative of DsbA2 or DsbA2 $\Delta \mathrm{N}$ covalent complexes with MalPEG5000, which adds 5,000 Da per cysteine. In E. coli, DsbA2 is maintained as the free thiol and $\mathrm{DsbA} 2 \Delta \mathrm{N}$ as the disulfide. 
(Jameson-Lee et al., 2011). As seen in Fig. 11, DsbA1 of L. pneumophila has a lag time of 20 min compared to DsbA2, which has a lag time of $5 \mathrm{~min}$, more typical of reductases like DsbC and thioredoxin. However, as a monomer, DsbA2LDN is much more active in this assay than DsbA1, though the time to reduction is doubled for the monomer compared with the dimer. These results suggest that monomeric DsbA2LDN, while structurally more similar to DsbA than DsbC, favors the reductase function (Jameson-Lee et al., 2011).

\subsection{Legionella pneumophila DsbA2, but not DsbA1 or DsbA2 $\Delta$ N, exhibits PDI activity}

In E. coli, DsbA catalyzes disulfide bonds between consecutive cysteine residues as the nascent polypeptide enters the periplasm (Kadokura et al., 2004). For those proteins for which nonconsecutive disulfide bonding is required for proper folding, consecutive disulfide bonds must be reduced and then reformed between nonconsecutive cysteine residues. In E. coli, the protein disulfide isomerase DsbC catalyzes this reaction (Ren \& Bardwell, 2011). In our previous studies, we noted that some of the proteins captured by DsbA2 contained an odd number of cysteine residues, and prediction software analyses suggested that some of these might be nonconsecutive (Jameson-Lee et al., 2011). Since DsbA2 exists as a homodimer and fully reduced in E. coli, we tested the possibility that DsbA2 might complement a $d s b C$ mutant of $E$. coli by restoring PDI activity. To test this hypothesis, $d s b A 2, d s b A 2 N$, and $d s b A 1$ were cloned into an $E$. coli strain carrying a $d s b C$ deletion and containing a pPDI detector system (Ren \& Bardwell, 2011). In this system, a TEM $\beta$-lactamase (Bla) which naturally contains two cysteine residues at positions 52 and 98 that are not required for biological activity was engineered to contain an additional two cysteine residues (81 and 108) positioned so that DsbA 


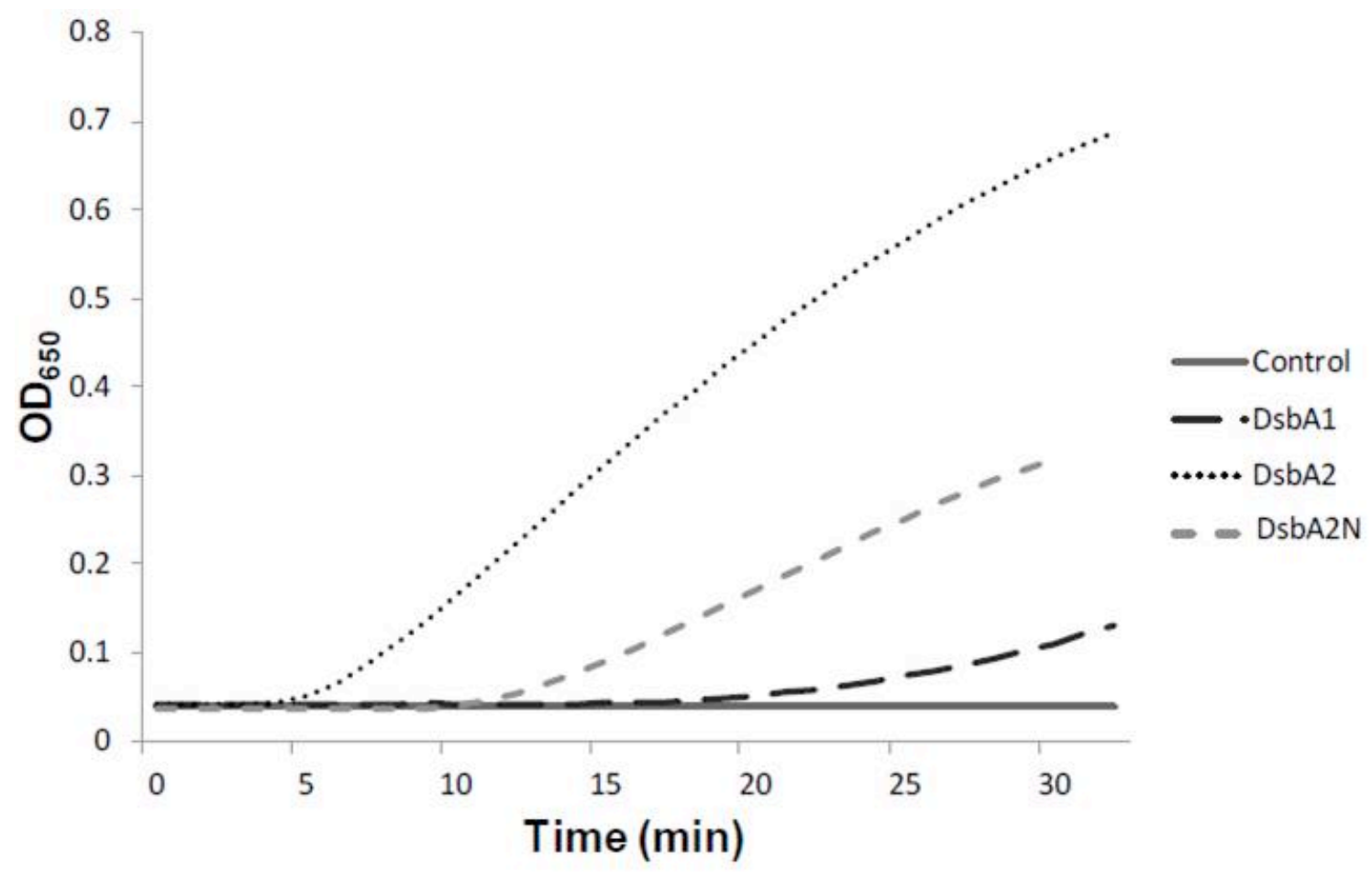

Figure 11. Insulin reduction assay. Reductase activities of equimolar concentrations of DsbA1, DsbA2, and DsbA2N (DsbA2 $\Delta \mathrm{N})$ were followed spectrophotometrically at $650 \mathrm{~nm}$ in a microplate assay as described above. The assays were performed in triplicate, and a representative assay is presented. The time to reduction indicates that $\mathrm{DsbA} 2 \Delta \mathrm{N}$ is intermediate between DsbA2 and DsbA1 (more reducing than oxidizing). 
will introduce consecutive disulfide bonds and produce an inactive enzyme (Ren \& Bardwell, 2011). In the presence of DsbC, biological activity is restored by PDI activity (Ren \& Bardwell, 2011). In this PDI detector assay, only $d s b A 2$ expression restored ampicillin resistance to nearly wild-type DsbC control levels at 2 or $3 \mathrm{~g} /$ liter of drug (Fig. 12A and B). In contrast, DsbA1 (depicted in Fig. 12A and B), DsbA2LDN (Fig. 12A), and DsbA2N (DsbA2 $\triangle$ N) (Fig. 12B) failed to complement in the PDI detector assay. The potent PDI activity displayed by DsbA2 in E. coli also suggests that DsbA2 is an efficient substrate of the DsbD reductase system, perhaps tipping the balance in favor of PDI/reductase activity over oxidase activity.

\subsection{DsbA2 exhibits PDI activity in vitro}

To confirm in vivo PDI activity for DsbA2, we developed an assay in which the PDI detector TEM $\beta$-lactamase serves as the substrate. In this assay, gain of Bla activity, as measured with the chromogenic substrate nitrocefin, is dependent on PDI enzyme reducing and shuffling disulfide bonds until correct nonconsecutive disulfides are formed (Hailu et al., 2013). As seen in Fig. 13, the Bla activity of MBla in concentrated spent culture supernatant is below the level of detection over the course of the assay. Incubation of DsbA2, but not DsbA1 with MBla efficiently corrected disulfide bonding and catalyzed protein folding, as noted by a gain in enzyme activity with nitrocefin. Compared with the WT Bla similarly concentrated from culture supernatants, near-equivalent activity was obtained. These studies were repeated several times, and results of a representative experiment are presented. These studies confirm the biological assay for PDI activity for DsbA2.

While L. pneumophila expresses a canonical DsbA (LpnDsbA1), the gene is neither essential for growth nor for virulence (will be discussed in detail from page 97) leaving 
A

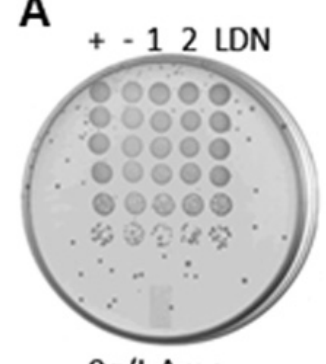

Og/L Amp
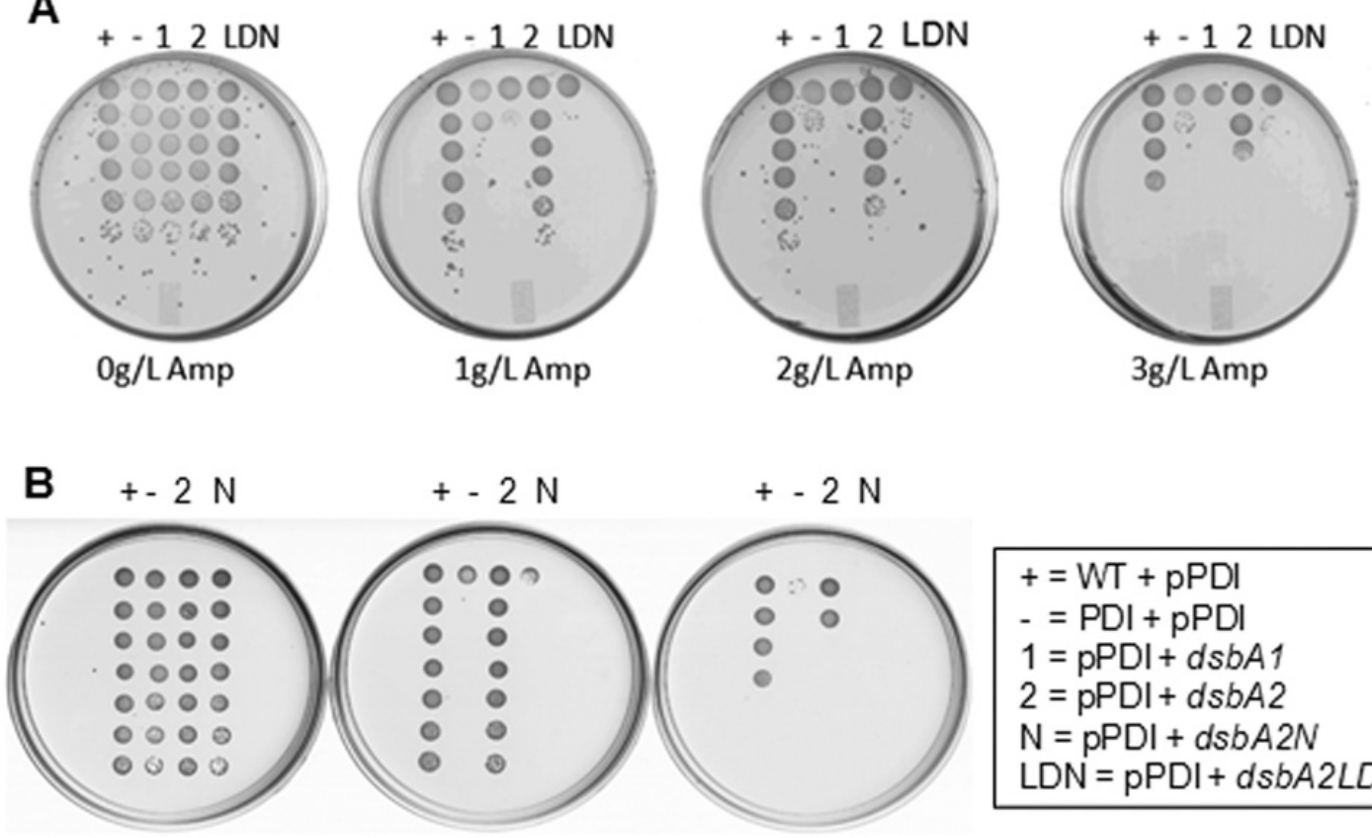

og/L Amp

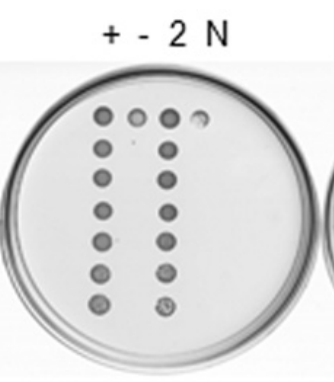

1g/L Amp

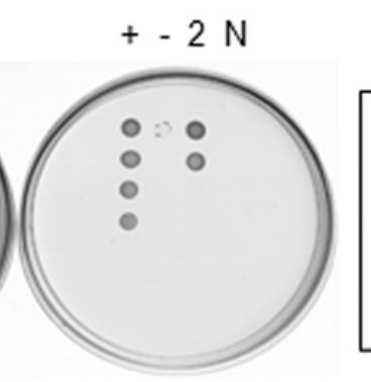

2g/L Amp
$+=\mathrm{WT}+\mathrm{pPDl}$

- = PDI + pPDI

$1=\mathrm{pPDI}+d s b A 1$

$2=\mathrm{pPDI}+d s b A 2$

$\mathrm{N}=\mathrm{pPDI}+d s b A 2 \mathrm{~N}$

$\mathrm{LDN}=\mathrm{pPDI}+d s b A 2 L D N$

Figure 12. Complementation tests in PDI detector system. The PDI detector system uses a $\beta$-lactamase engineered to form consecutive disulfide bonds, which require DsbC PDI activity to correct to nonconsecutive to facilitate protein folding and gain of enzyme activity (Ren \& Bardwell, 2011). Growth on ampicillin indicates that $\beta$-lactamase is properly folded. Ampicillin concentrations are $0,1,2$, or $3 \mathrm{~g} /$ liter. Bacterial dilutions are plated from top to bottom as detailed in the text. $(A)+$, wild-type E. coli strain RGP663 plus pPDI detector plasmid; - , strain RGP665dsbC plus pPDI detector plasmid; 1, strain RGP665 plus pPDI detector plasmid plus pBCdsbA1; 2, strain RGP665 plus pPDI detector plasmid plus pBCdsbA2; LDN; strain RGP665 plus pPDI detector plasmid plus pBCdsbA2LDN (LDN, deleted leader and dimerization domain sequences). (B) Replicate PDI detector assay that includes RPG665 plus pPDI detector plasmid plus $\mathrm{pBC} d s b A 2 N(\mathrm{pBC} d s b A 2 \Delta N)$ containing the leader sequence and an in-frame deletion of the dimerization domain sequences. 


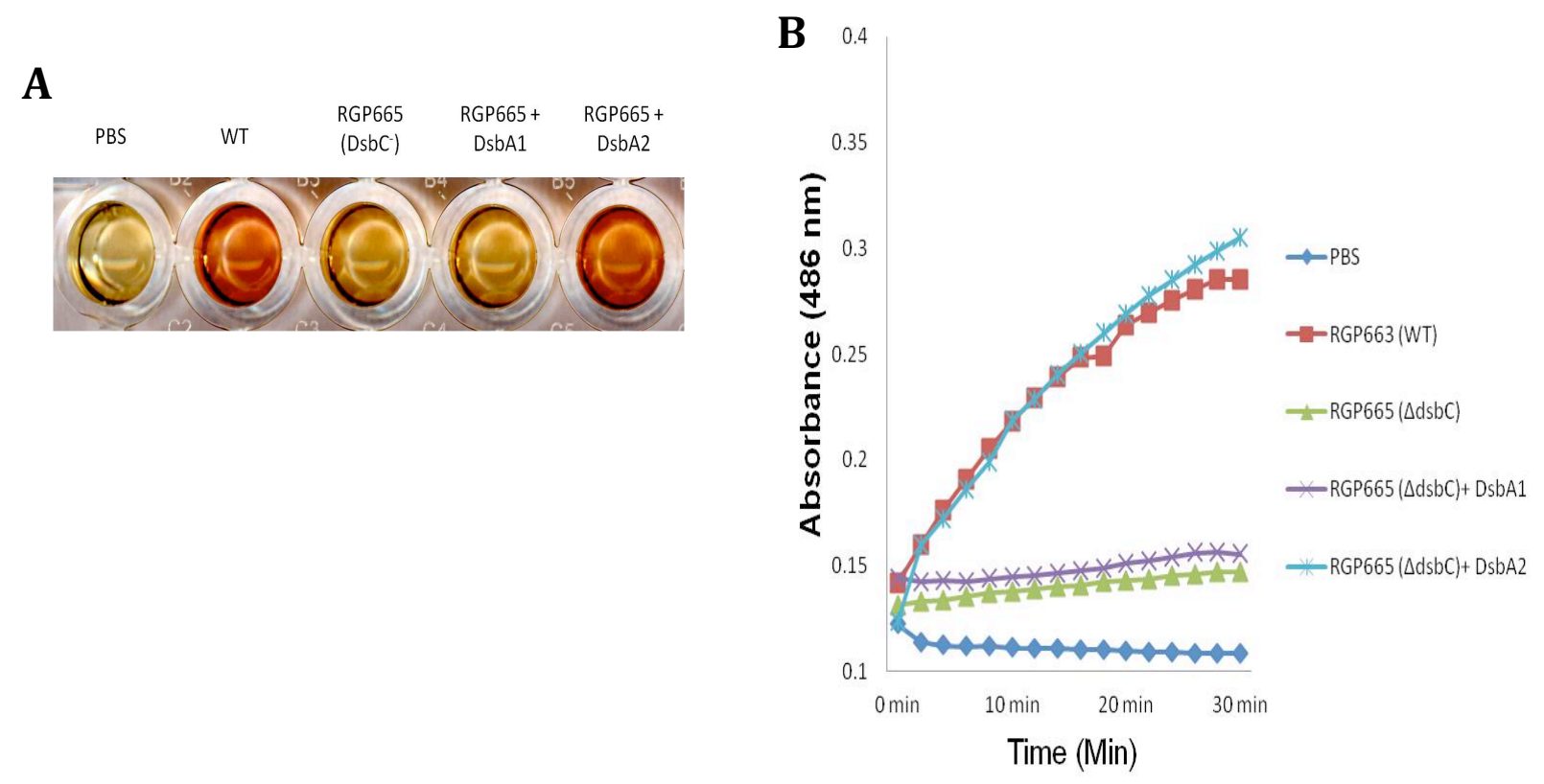

Figure 13. Nitrocefin PDI folding assay. TEM1 $\beta$-lactamase (Bla) and pPDI mutant TEM1 $\beta$ lactamase (MBla) were obtained by concentrating culture supernatants from strains RGP663 and RGP665, respectively. MBla contains consecutive disufide bonds and is inactive (nitrocefin negative). DsbA1 or DsbA2 was added to aliquots of MBla for $30 \mathrm{~min}$ at $37^{\circ} \mathrm{C}$, and $15 \mu \mathrm{l}$ aliquots were tested in triplicate for Bla activity in microplates with nitrocefin as described in the text. A) Cleavage of the $\beta$-lactam of nitrocefin is monitored as a change in color over $30 \mathrm{~min}, \mathbf{B}$ ) rates of hydrolysis are plotted by the increase in absorbance at $486 \mathrm{~nm}$ over time in micro-titer plate. DsbA2, but not DsbA1, exhibited PDI activity. 
homodimeric DsbA2 to catalyze both sequential disulfide bond formation in nascent polypeptides and conversion of consecutive or inappropriate disulfide bonds into non-consecutive disulfide bonds through protein disulfide isomerase (PDI) activity to promote correct protein folding (Jameson-Lee et al., 2011; Kpadeh et al., 2013). Evaluation of the redox status of DsbA2 in $L$. pneumophila revealed an equilibrium between the free thiol $(\mathrm{SH})$ and the disulfide (S-S) forms while in E. coli, DsbA2 was exclusively maintained in the reduced thiol form (Kpadeh et al., 2013). This suggests that in L. pneumophila the activities of partner proteins DsbB that typically re-oxidize DsbA and DsbD that typically reduces DsbC, according to the E. coli paradigm, must cooperate to maintain equilibrium of redox forms.

\subsection{Identification and nomenclature of DsbB and DsbD proteins}

Analysis of the complete sequenced genomes of L. pneumophila and related species reveals multiple alleles of DsbB and DsbD. In the Philadelphia 1 strain, one DsbB is annotated as LidJ (lethal in Dot) as it was selected as non-essential in a dotA mutant background (Conover et al., 2003). Comparative amino acid sequence analysis of LidJ with DsbB of E. coli reveals both similarity and divergence (see Figure 14). In our studies LidJ is referred to as DsbB2. One of the unique features of DsbB2 is a unique periplasmic domain containing a thioredoxin fold (see Figure 14). A second DsbB in the Philadelphia-1 strain is mis-annotated as DsbH. In all other sequenced strains, a canonical DsbB is present and shows a high degree of similarity with the DsbB of E. coli (see Figure 14 alignment). A re-examination of the $d s b H$ locus in the Philadelphia-1 strain reveals the complete gene is present, which we verified by functional complementation studies in E. coli and named this allele DsbB1 (figure 16). 


\section{S---S}

LpnDsbB2

LpnDsbB1

EcDsbB

LpnDsbB2

LpnDsbB1

EcDsbB

LpnDsbB2

LpnDsbB1

EcDsbB

LpnDsbB2

LpnDsbB1

EcDsbB

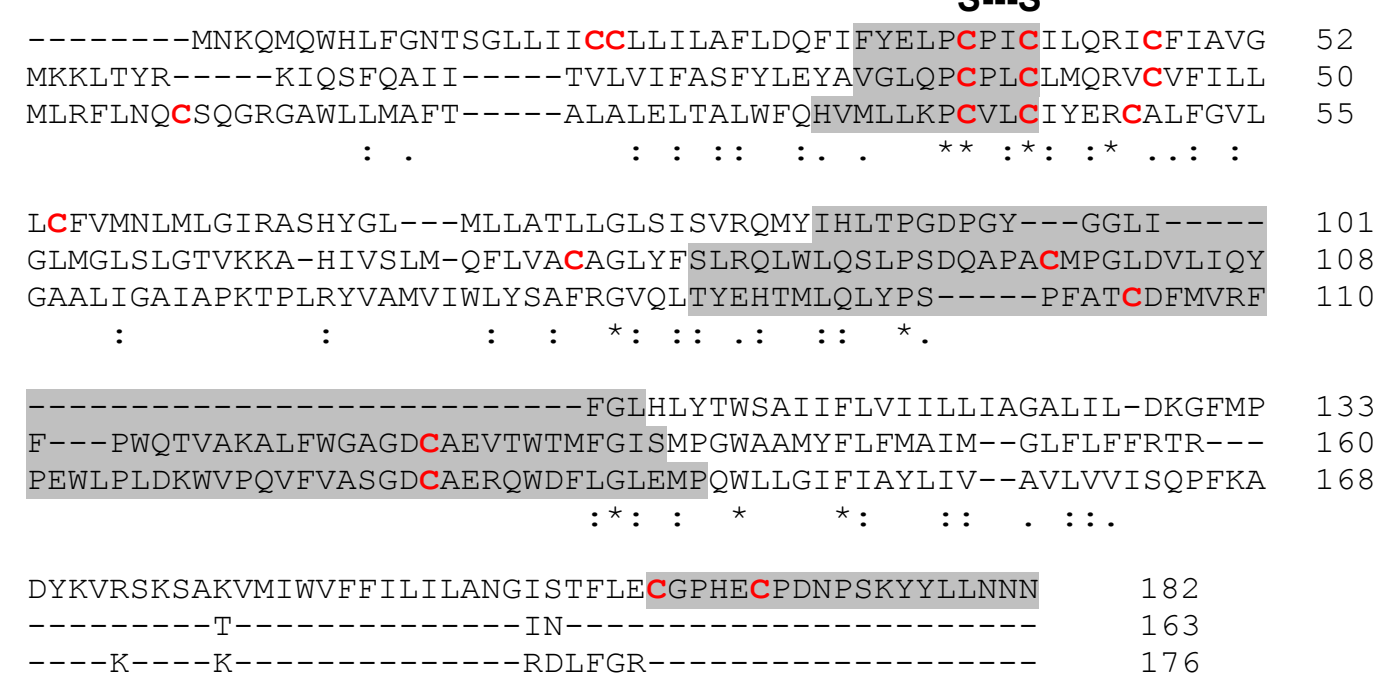

--------MNKQMQWHLFGNTSGLLI ICCLLILAFLDQFIFYELPCPICILQRICFIAVG 52 MKKLTYR-----KIQSFQAII-----TVLVIFASFYLEYAVGLQPCPLCLMQRVCVFILL 50 MLRFLNQCSQGRGAWLLMAFT-----ALALELTALWFQHVMLLKPCVLCIYERCALFGVL 55

LCFVMNLMLGIRAS HYGL---MLLATLLGLS ISVRQMY I HLTPGDPGY---GGLI----- 101 GLMGLSLGTVKKA-HIVSLM-QFLVACAGLYFSLRQLWLQSLPSDQAPACMPGLDVLIQY 108 GAALIGAIAPKTPLRYVAMVIWLYSAFRGVQLTYEHTMLQLYPS-----PFATCDFMVRF 110

-----------------------EGLHLYTWSAIIFLVIILLIAGALIL-DKGFMP 133 F---PWQTVAKALFWGAGDCAEVTWTMFGISMPGWAAMYFLFMAIM--GLFLFFRTR--- 160 PEWLPLDKWVPQVFVASGDCAERQWDFLGLEMPQWLLGIFIAYLIV--AVLVVISQPFKA 168

DYKVRSKSAKVMIWVFFILILANGISTFLECGPHECPDNPSKYYLLNNN 182

$----\mathrm{K}----\mathrm{K}-------------\mathrm{RDLFGR}------------------176$

Figure 14. Clustal O Alignment of DsbB proteins. The cysteine residues are bold red and the grey highlighted regions represent regions extending into the periplasm. The S-S identifies cysteine residues that form a disulfide bond. 
Similarly, L. pneumophila expresses two alleles of $d s b D, d s b D 1$, as depicted in Fig. 15, which is also annotated as lidC and located just upstream of the groELS operon. The second $\operatorname{DsbD}(\operatorname{Lpg} 1680)$ is referred to as DsbD2 and is located elsewhere. While Conover et al. (2003) were able to obtain lidJ $(d s b B 2)$ and $l i d C(d s b D 1)$ mutants, our attempts to generate these mutants in L. pneumophila Lp02 and in the AA100 backgrounds have not been successful. In our studies we used either $\mathrm{Km}$ or $\mathrm{Gm}$ cassettes inserted in-frame within the truncated open reading frame (orf) such that mutations were non polar on downstream genes. In these studies, we were routinely able to produce $d s b A l$ mutants, which we had previously reported to be non-essential (Jameson-Lee et al., 2011). In all studies, we were not successful in obtaining $d s b A 2$ mutants as previously reported (Jameson-Lee et al., 2011). While a TraSH mutant screen performed in rich medium with the Lp02 strain confirms the essentiality of $d s b A 2, d s b B 1$ could not be evaluated ( $d s b H$ orf incorrect) and both $d s b D 1$ and $d s b D 2$ were borderline and lidJ (dsbB2) was called as non-essential (Conover et al., 2003). Of interest though is the apparent lack of functional redundancy, suggesting that the DsbB and DsbD alleles might interact with different periplasmic proteins whose functions are distinct. To begin testing function for each of the $d s b$ alleles, we used a complementation approach in a set of defined E. coli $d s b$ mutants. In this strategy, DsbA function was evaluated by gain of motility in a soft agar motility assay. DsbA is required for formation of a disulfide bond in FlgI, a motor protein required for flagellar function. To assess protein disulfide isomerase function, we employed an in vivo folding assay developed by Ren and Bardwell and as described by us (Ren and Bardwell, 2011; Kpadeh et al., 2013). 


\begin{tabular}{|c|c|c|}
\hline CDsbD & $------M A Q R I F T L I L L L C S T S V F A G L F D A P G R S Q F V P A D Q A F A F D F-Q Q N Q H D L N$ & 49 \\
\hline DsbD2 & ------------------------------------------------------------ & 0 \\
\hline DsbD1 & MFISNSKRYRMKKWLLFSLLFLTSLCVH--------AEPLPASEVFKVSVKKIDPNTFA & 51 \\
\hline EcDsbD & LTWQIKDGYYLYRKQIRITPEHAKIAD---VQLPQGVWHEDEFYGKSEIYRDRLTLPVTI & 06 \\
\hline DsbD2 & ------------------------------------------------------------ & \\
\hline \multirow[t]{2}{*}{ DsbD1 } & IQWNILPKYFLYSDRIQLNSDNDDIAQLGTLRFPTPLTKTDKQGRTFKVYRNQLNLPVGV & 11 \\
\hline & No disulfide bond region & \\
\hline EcDsbD & NQASAGAT-LTVTYQGCADAGFCYPPETKTVPLS-------EVVANN-----EASQPVS & 152 \\
\hline DsbD2 & ---------- MLFITY-------WDIK-MRKLIDSFMILLLIFFTQAAWSYTPGFESS & 0 \\
\hline \multirow[t]{2}{*}{ DsbD1 } & LGITPGETIVNLRFQGCADDGFCYPPEVKQIKLAIDDKLALSQV--DLETFHAPEATPLE & 169 \\
\hline & $\begin{array}{lllll}:: & : & : & 0\end{array}$ & \\
\hline EcDsbD & VPQQEQPTAQLPFSALWA------LLIGIGIAFTPCVLPMYPLISGIVLGGKQRLSTARA & 06 \\
\hline DsbD2 & NPD-TVM--HFITGNSAFVYLSAFFGLGILLAFTPCVLPMVPILSGIIVGQNSI-STGKA & 6 \\
\hline \multirow[t]{2}{*}{ DsbD1 } & KPQQDIA--DIFANHNWIMILLIFYGFGLLLSFTPCILPMVPVLSGIIVGHGKTATTKKA & \\
\hline & 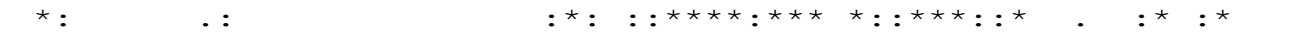 & \\
\hline EcDsbD & LLLTFIYVQGMALTYTALGLVVAAAGLQFQAALQHPYVLIGLAIVFTLLAMSMFGLFTLQ & 66 \\
\hline DsbD2 & FKLSLSYVTGMAITYAGAGMLAGYMGSTIQTVMQRPVVITLFSVVFVAMALSMFGFFELK & 56 \\
\hline \multirow[t]{2}{*}{ DsbD1 } & FFLSLSYVLSMSVTYAVVGAVVALLGANLQISMQSPWAISLFSLIFVLLALSMFGFYEFK & 287 \\
\hline & 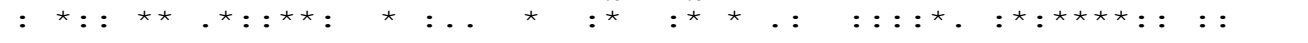 & \\
\hline EcDsbD & LPSSLQTRLTLMSNRQQGGSPGGVFIMGAIAGLICSPCTTAPLSAILLYIAQSGNMWLGG & 326 \\
\hline DsbD2 & FPTSLGNRLNKNNQLGKQRSYLSVGIMGVLSTLIVSPCVTAPLIGVLSYIGQNGQVIMGG & 216 \\
\hline \multirow[t]{2}{*}{ DsbD1 } & LPDAWQSKIVGSSREQRGGHYLGAAIMGCLSTLILSPCVTAPLIGVLTYIAQTGNVLLGS & 347 \\
\hline & 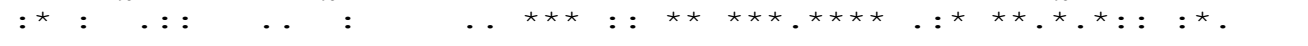 & \\
\hline EcDsbD & GTLYLYALGMGLPLMLITVFGNRLLPKSGPWMEQVKTAFGFVILALPVFLLERVIGDIWG & 386 \\
\hline DsbD2 & LILFVMALGMGVPLLIVGAGYGTLLPKAGSWMLKIKQLFGFIMLGIAIWMLSRILNQTIT & 276 \\
\hline \multirow[t]{2}{*}{ DsbD1 } & VTLFTLSLGMGTPLLLIGTSAGKWLPETGSWMNAVKAFFGILLLAVAIYLMARILPAGLV & 407 \\
\hline & 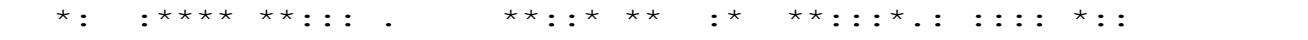 & \\
\hline EcDsbD & LRLWSALGVAFFGWAFITSLQAKRGWMRV-----------VQIILLAAALVSVRPLQDW & \\
\hline DsbD2 & HILWAGLLM--VASISLGALKSQNNWRGYLAQGLGILALISGGIIFYNTAIPSVK----- & 329 \\
\hline \multirow[t]{3}{*}{ DsbD1 } & MGLWACLLI--FSGIYSGALTKSNTNQEKLCQGIGIILLTYGLLILIGASMGSSNPLQPL & 465 \\
\hline & $\star \star: \quad \star \quad: \quad \ldots$ & \\
\hline & $\mathrm{C} \gamma$ disulfide bond reg: & \\
\hline EcDsbD & AFGATHTAQTQTHLNFTQIKT--VDELNQALVEAKGKPVMLDLYADWCVACKEFEKYTFS & 492 \\
\hline DsbD2 & --IKQA---SVENSPFIKINTLNALQLQLEQAKKVRKAVFLDFSADWCGDCQEMDTKVFN & 384 \\
\hline \multirow[t]{2}{*}{ DsbD1 } & ANLQAA---PTVSNAFESAKAQSVKSVELAIKQAFGKPVMLDFYADWCASCKVMENTTFK & 522 \\
\hline & $\star \star: \star \star: \star \star \star \star \quad \star::::{ }^{\star}{ }^{\star}$ & \\
\hline EcDsbD & DPQVQKALADTVLLQANVTANDAQDMALLKHLNVLGLPTILFFDGQGQEHPQARVTGFMD & 52 \\
\hline DsbD2 & QPEIQKAMSDWVSIKVDISDKNAEVDSIKKAFGIYGTPTMIFFNTSGEPITQMTSVGLIS & 4 \\
\hline \multirow[t]{2}{*}{ DsbD1 } & DPRVQKALSHFIVIKVDVTANNKNDKALMQHFRVVAPPTFIFFNADGVQLNNLKRVGELN & \\
\hline & 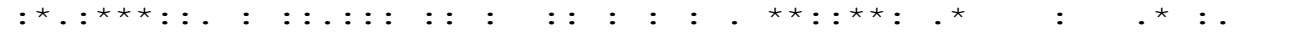 & \\
\hline EcDsbD & AETFSAHLRDRQP-- & \\
\hline DsbD2 & LSKMLQLLEQARHLS & \\
\hline DsbD1 & ADEFMQTIKSIDEN- & \\
\hline
\end{tabular}

Figure 15. Clustal 0 Alignment of DsbD proteins. The cysteine residues are bold red, depicting the $\mathrm{N}$-terminal $\alpha$-disulfide bond region and $\mathrm{C}$-terminal $\gamma$-disulfide bond region with conserved amino acids, shaded regions. 


\subsection{Evaluation of DsbB1 and DsbB2 in E. coli $d s b B$ mutants}

We first determined whether $d s b B 1$ and $d s b B 2$ could functionally complement a $d s b B$ mutant of $E$. coli by evaluating strains for motility in soft in soft agar. Our results reveal that both Lpn DsbB1 and DsbB2 expressed in RGP405 strain were able to partially restore motility compared to wild type (JP114), as shown in (Fig. 16). This indicates that Lpn DsbBs are capable of re-oxidizing DsbA leading to partial gain of motility.

\subsection{Both Lpn DsbB1 and DsbB2 interact with monomeric DsbA2 (DsbA2 $\Delta N)$ in E. coli}

DsbA2 exhibits both oxidase activity (Jameson-Lee et al., 2011) and isomerase activity (Kpadeh et al., 2013), but until now its interacting partners have been unidentified. We sought to elucidate the ability of either DsbB1 or DsbB2 in oxidizing DsbA2 using an E. coli $d s b A d s b B$ double mutant (RGP438), and then assess motility. We overexpressed Lpn DsbA2 and Lpn DsbB1 or Lpn DsbA2 and Lpn DsbB2 in an E. coli double mutant $(\Delta d s b A d s b B)$ background. Interestingly, neither DsbB1 nor DsbB2 were able to restore motility in a DsbA2 producing strain (Figure 17B). Most importantly, $d s b A 2:: d s b B 2$ construct was toxic and unable to show any measurable growth in broth upon induction after many trials. The toxicity may indicate the functional difference of Lpn DsbB2 due to its structural difference from Lpn DsbB1 and EcDsbB, as shown in Figure 14. To further investigate the importance of Lpn DsbBs existence in $L$. pneumophila, we decided to investigate the interaction of monomeric DsbA2 (DsbA2 $\Delta \mathrm{N})$, which has been reported to complement the function of Ec $d s b A$ in a motility assay (Kpadeh et al., 2013), suggesting it is a substrate of DsbB for re-oxidation. As seen in Figure 17A, wild-type E. coli strain (JP114) is motile in $0.4 \%$ soft agar, whereas $\triangle d s b A d s b B$ double mutant strain 


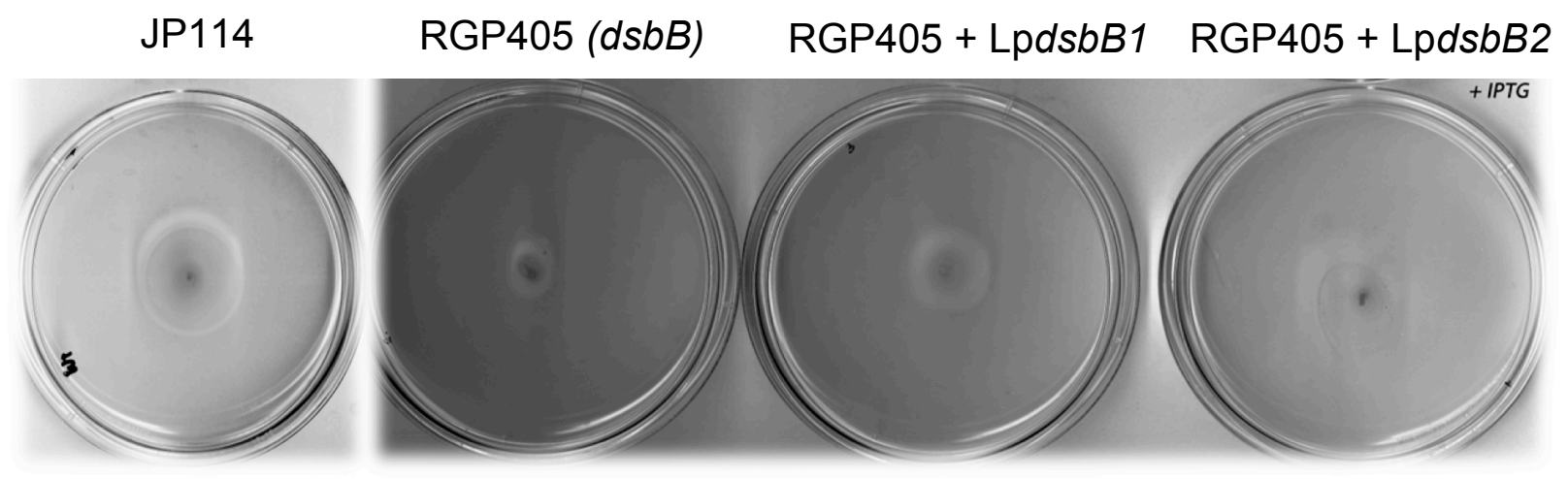

Figure 16. Lpn DsbB1 and Lpn DsbB2 complement motility in an E. coli dsbB mutant strain. Spreading motility by $E$. coli is the measured of chemotaxis, which is dependent on the proper assembly and function of flagella motor protein. Left: Wild type E. coli (JP114) is motile in soft agar and forms a large zone of growth, while $d s b B$ mutant strain RGP405 is non-motile and grows only where it is stabbed into the center of the plate. Addition of either Lpn DsbB1 or Lpn DsbB2 in a plasmid is able to functionally replace $E$. coli $d s b B, \sim 30 \%$ and $\sim 45 \%$, respectively, as demonstrated by the large zone of growth. The results are representative of typical results obtained from triplicate platings. 
RGP438 is not. We introduced DsbA2 $\triangle \mathrm{N}$ with either L. pneumophila $d s b B 1$ or $d s b B 2$, and coexpressed from pBC plasmids into RGP438 to test whether expression of these proteins could restore motility. As depicted in Figure 17A, $d s b A 2 \Delta \mathrm{N}:: d s b B 1$ and $d s b A 2 \Delta \mathrm{N}:: d s b B 2$; and also as depicted in Figure 17B, $d s b A 1:: d s b B 1$ and $d s b A 1:: d s b B 2$ complemented motility to $\sim 30$ and $50 \%$ to that of wild-type, respectively. Growth/motility is measured by the diameter of spreading. Note that the differences in growth rate of bacterial containing E. coli proteins to bacterial containing a mixture of L. pneumophila and E. coli proteins might account for the partial complementation noted in our studies. These results further validate the consistency of the general view that dimeric proteins, such as DsbC or DsbA2 (containing the dimerization domain), are poor substrates of DsbB (Bader et al., 2001; Segatori et al., 2004; Shouldice et al., 2011; Kpadeh et al., 2013). Therefore, we suggest that it is the dimerization domain of DsbA2 that prevents or restricts interaction of the homodimer with DsbB as previously indicated in (Kpadeh et al., 2013). Therefore, we decided to investigate the interaction of Lpn DsbA2 with the reductants (DsbD1 and DsbD2).

\subsection{Lpn DsbD1 and Lpn DsbD2 are unique for DsbA2 bifunctional activity}

In E. coli, the DsbA-DsbB pathway introduces disulfide bonds de novo, while the DsbCDsbD pathway functions to isomerize disulfides. In order to investigate the DSB activity of the different DsbDs in L. pneumophila, we sought to establish the role of Lpn DsbD1 and Lpn DsbD2 as reductants by complementing E. coli $d s b D$ mutant (RGP666) using a PDI detector assay developed by (Ren and Bardwell, 2011; Kpadeh et al., 2013). In this assay, the reductant role of DsbD is important for the isomerase activity of DsbC. In order to test Lpn DsbD1 or 


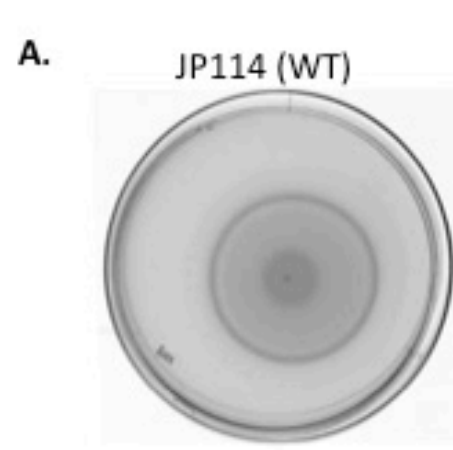

RGP438
RGP438

$(\triangle d s b A \Delta d s b B)$

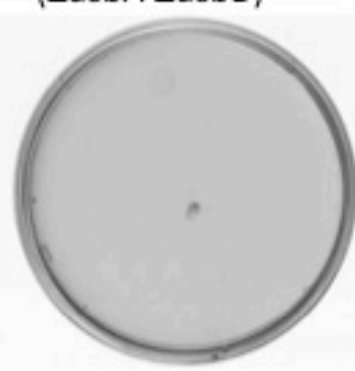

RGP438
RGP438

$(p d s b A 2 \Delta N+\mathrm{pdsbB1})$

B. $\quad\left(\begin{array}{llll}\text { p } d s b A 1+p d s b B 1) & (p d s b A 1+p d s b B 2) & (p d s b A 2+p d s b B 2)\end{array}\right.$

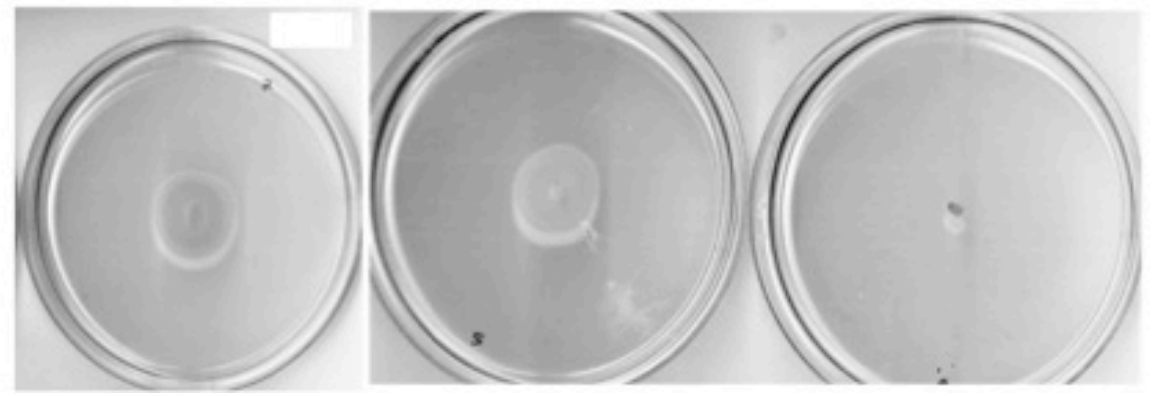

Figure 17. Both Lpn DsbB1 and DsbB2 interact with monomeric DsbA2 (DsbA2 $\Delta N)$ in $E$.

coli. Lpn DsbA1 and monomeric DsbA2 (DsbA2 $\Delta \mathrm{N})$, but not homodimeric DsbA2, interact with both Lpn DsbB1 and Lpn DsbB2 to complement motility in an E. coli dsbA dsbB mutant strain. We assessed the spreading motility by E. coli as in Fig. 3. Wild type E. coli (JP114) are motile in soft agar and form a large zone of growth, while $d s b A d s b B$ mutant strain RGP438 is non-motile and grows only where it was stabbed into the center of the plate. Addition of plasmids encoded (A) Lpn DsbA2 $\Delta \mathrm{N}$ and (B) Lpn DsbA1 with either Lpn DsbB1 or Lpn DsbB2 added to RGP438 are able to functionally replace $E$. coli $d s b A d s b B$, but (B) Lpn DsbA2 with either Lpn DsbB1 or Lpn DsbB2 on a plasmid did not restore motility in RGP438 as demonstrated in this figure. Results shown here are representation of a single experiment obtained from triplicate platings. 
Lpn DsbD2 role in contributing to EcDsbC function, we expressed either Lpn DsbD1 or Lpn DsbD2 from inducible plasmid constructs in RGP666 and then tested their ability to maintain $\mathrm{EcDsbC}$ in the reduced form. Both Lpn DsbDs were unable to interact with EcDsbC to complement $\mathrm{Ec} d s b D$ mutant for isomerase activity as shown in Figure 18A (lanes $3 \& 4$ ). However, co-expression of $d s b A 2:: d s b D 1$ or $d s b A 2:: d s b D 2$ in RGP666 restored PDI activity as measured by growth on Amp plates (Fig. 18A, lanes $5 \& 6$ ), suggesting a unique affinity of both Lpn DsbD1 and Lpn DsbD2 for DsbA2, and not for EcDsbC. In order to validate the interaction of DsbD1 and DsbD2 with DsbA2 was specific we utilized a $d s b C d s b D$ double mutant (RGP810) of E. coli. As shown in Figure $18 \mathrm{~B}$ expression of either $d s b A 2:: d s b D 1$ or $d s b A 2:: d s b D 2$ constructs in RGP810 (lane 5 \& 6) restored TEM-1 $\beta$-lactamase function, indicating that both DsbD1 and DsbD2 control the reduction of DsbA2 in L. pneumophila, revealing a novel interaction of DsbA-like protein interaction with a reductant; contrary to the E. coli DsbC/DsbD "classical" pathway. In addition, as depicted in Figure 15, E. coli DsbD differs from both Lpn DsbD1 and Lpn DsbD2. EcDsbD $\alpha$-disulfide bond region, which is located in the periplasmic region appears earlier (around aa position 123-129) while in Lpn DsbD1 it begins at aa position 128-134, and this conserved region seems to be missing in Lpn DsbD2 (Fig. 35). While the conserved $\gamma$-disulfide bond region seems to be conserved amongst these DsbDs, Lpn DsbDs seem to show some diversity and or differences in the $\beta$-disulfide region compared to EcDsbD; thereby contributing to their inability to reduce EcDsbC as observed in the isomerase assay. All together, these results indicate that both DsbD1 and DsbD2 are structurally diverse from EcDsbD; suggesting a moderate interaction of Lpn DsbDs with DsbA2 is needed for DsbA2 to exist in a bifuntional state in vivo, and the fully reduced status of DsbA2 as reported in (Kpadeh et al., 2013) 


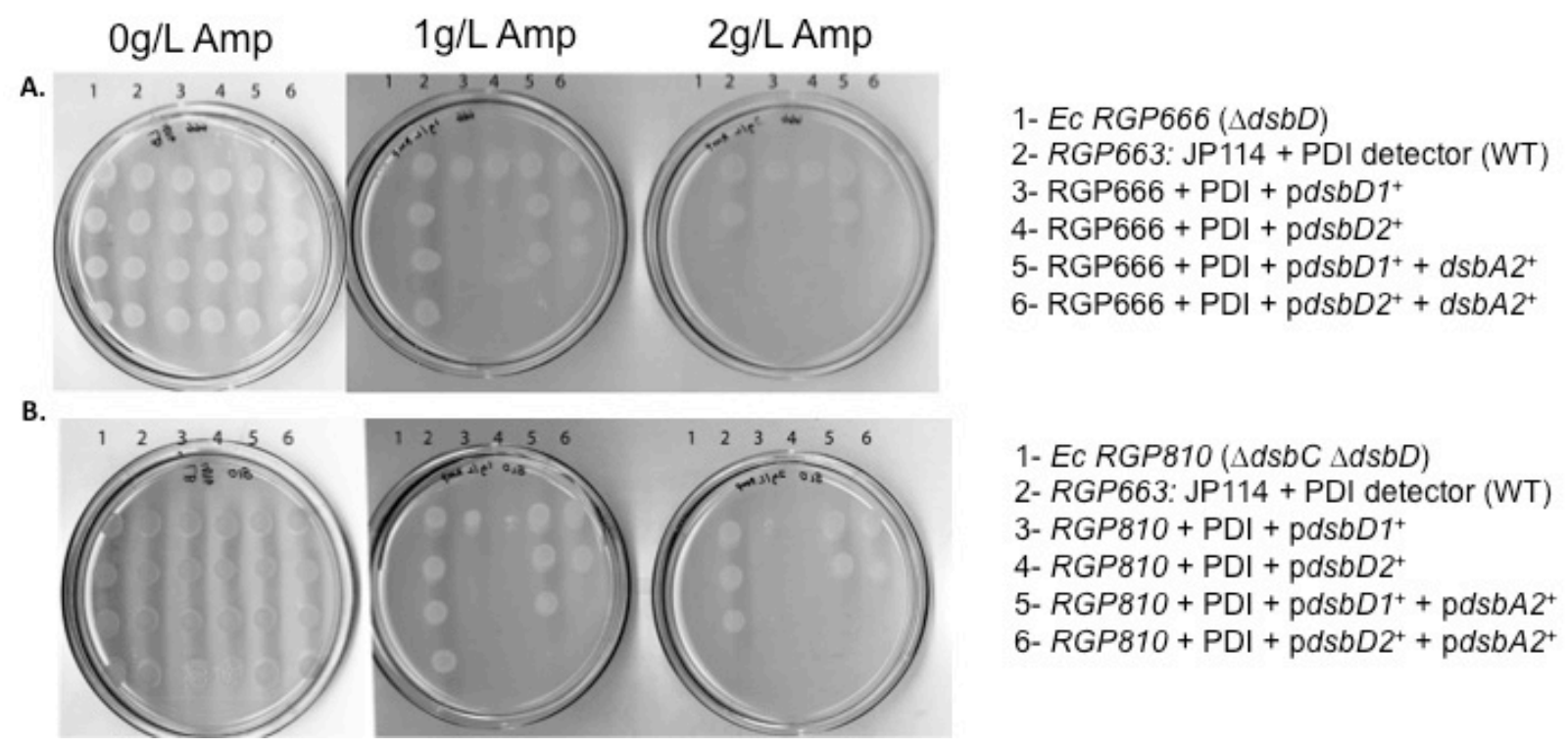

Figure 18. Isomerization activity in a PDI detector assay. Protein isomerase activity is required for the proper folding and function of those proteins that require nonconsecutive disulfide folding. We utilized $\beta$-lactamase engineered to form consecutive disulfide bonds, which require DsbC (reductase) and/or DsbD (reductant; maintains DsbC in the reduced form) PDI activities to correct the mis-disulfides to nonconsecutive to facilitate protein folding resulting to an active enzyme (Ren et al., 2011; Kpadeh et al., 2013). Growth on ampicillin demonstrates $\beta$ lactamase is properly folded and active. Ampicillin concentrations are 0,1 , or $2 \mathrm{~g} /$ liter. Bacteria were serially diluted with concentrations ranging from top $\left(10^{9}\right)$ to bottom $\left(10^{3}\right)$. (A) Lpn DsbD1 and Lpn DsbD2 do not interact with E. coli DsbC (lanes 3 \& 4). (A \& B) Both Lpn DsbD1 and Lpn DsbD2 interact with Lpn DsbA2 (lanes 5 \& 6). Experiments were performed three times independently. Reported here is a representative of the results obtained from single experiment performed in triplicate. 
is due to the EcDsbD efficiently reducing DsbA2 while preventing Lpn DsbBs interaction with DsbA2.

\subsection{DsbA2 exhibits oxidase activity in the presence of Lpn $d s b B s$ and Lpn $d s b D s$}

So far, we have identified the DSB interacting partners of the different forms of Lpn DsbA2. Hence, the genetically constructed monomer form of DsbA2 seems to be active and functional. However, this form does not exist in nature. We sought to evaluate the hypothesis that Lpn DsbBs and DsbDs moderately interact with DsbA2 to produce the mixture of oxidized and reduced redox forms, with the oxidized form contributing to a DsbA-like activity in $L$. pneumophila, as reported in (Kpadeh et al., 2013). Utilizing $\Delta d s b A \Delta d s b B$ double mutant strain RGP438, we chromosomally replaced E. coli $d s b D$ with Lpn $d s b D 1$ or Lpn $d s b D 2$ creating ZK01 and ZK02 by PCR as described in the method section. Expression of pMMB206dsbA2:dsbB1 or pMMB206dsbA2:dsbB2 in either ZK01 or ZK02 was able to restore motility as shown in Figure 19. We then evaluated the redox status of DsbA2 using the above genetically altered constructs that were motile. As seen in Figure 20, DsbA2 with E. coli $d s b B$ and $d s b D$ genes was fully reduced (lane 3), while in the presence of both Lpn $d s b B$ and Lpn $d s b D$ (lane 6), DsbA2 is shown to exhibits a mixture of oxidized and reduced redox forms in E. coli; similar to what is biological reported in L. pneumophila (Kpadeh et al., 2013). These results indicate that when both Lpn DsbD proteins are absent, Lpn DsbA2 accumulates mostly in the reduced form in the presence of Ec DsbD (Fig. 20; lane 3), suggesting Lpn DsbDs role are essential for DsbA2 to exist as oxidized in the periplasm. In addition, these results established that DsbA2 is a bifunctional protein; indicating oxidative and reductive pathways. This research challenges the E. coli DsbA/DsbC paradigm established in the field and introduces a novel single 


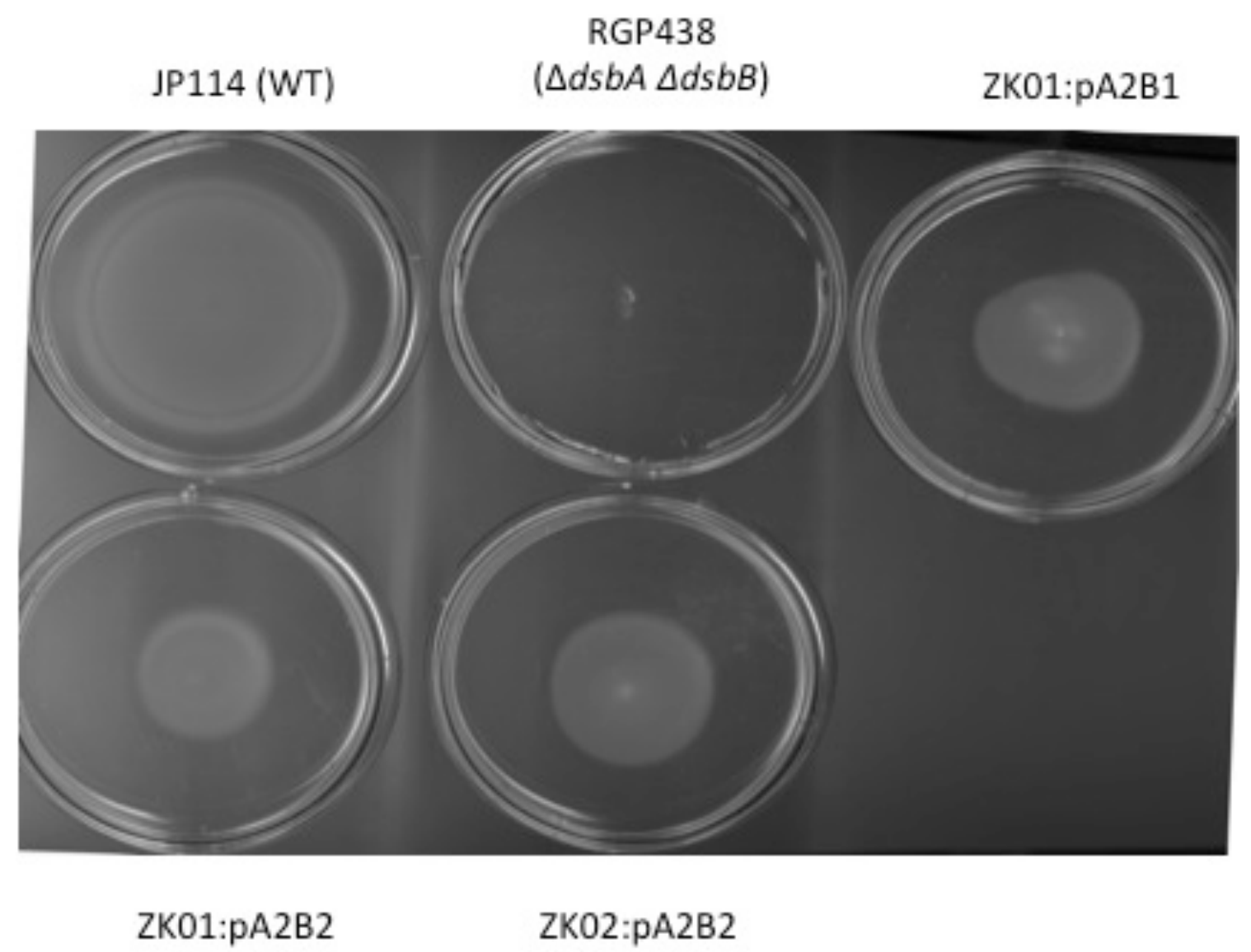

Figure 19. Lpn DsbA2 exhibits oxidase activity when both Lpn DsbB and Lpn DsbD are present. In order to investigate the oxidase activity of DsbA2 in the presence of Lpn DSB proteins, we chromosomally replaced $E$. coli $d s b D$ with either $L p n d s b D 1$ or $L p n d s b D 2$ resulting to genetically altered strains ZK01 and ZK02, respectively. We then complemented E. coli dsbA $d s b B$ with Lpn DsbA2, and then added either Lpn DsbB1 or Lpn DsbB2 (constructs including $d s b A 2, d s b B 1$ or $d s b B 2$ require IPTG). We assessed the spreading motility by $E$. coli as in Fig. 3. Addition of plasmids encoding Lpn $d s b A 2$ and Lpn $d s b B 1$ or Lpn $d s b B 2$ with either chromosomally-replaced $\mathrm{Lpn} d s b D 1$ or $\mathrm{Lpn} d s b D 2$ in RGP438 were able to functionally complement an $E$. coli $d s b A d s b B$ double mutant to restore motility as demonstrated. 


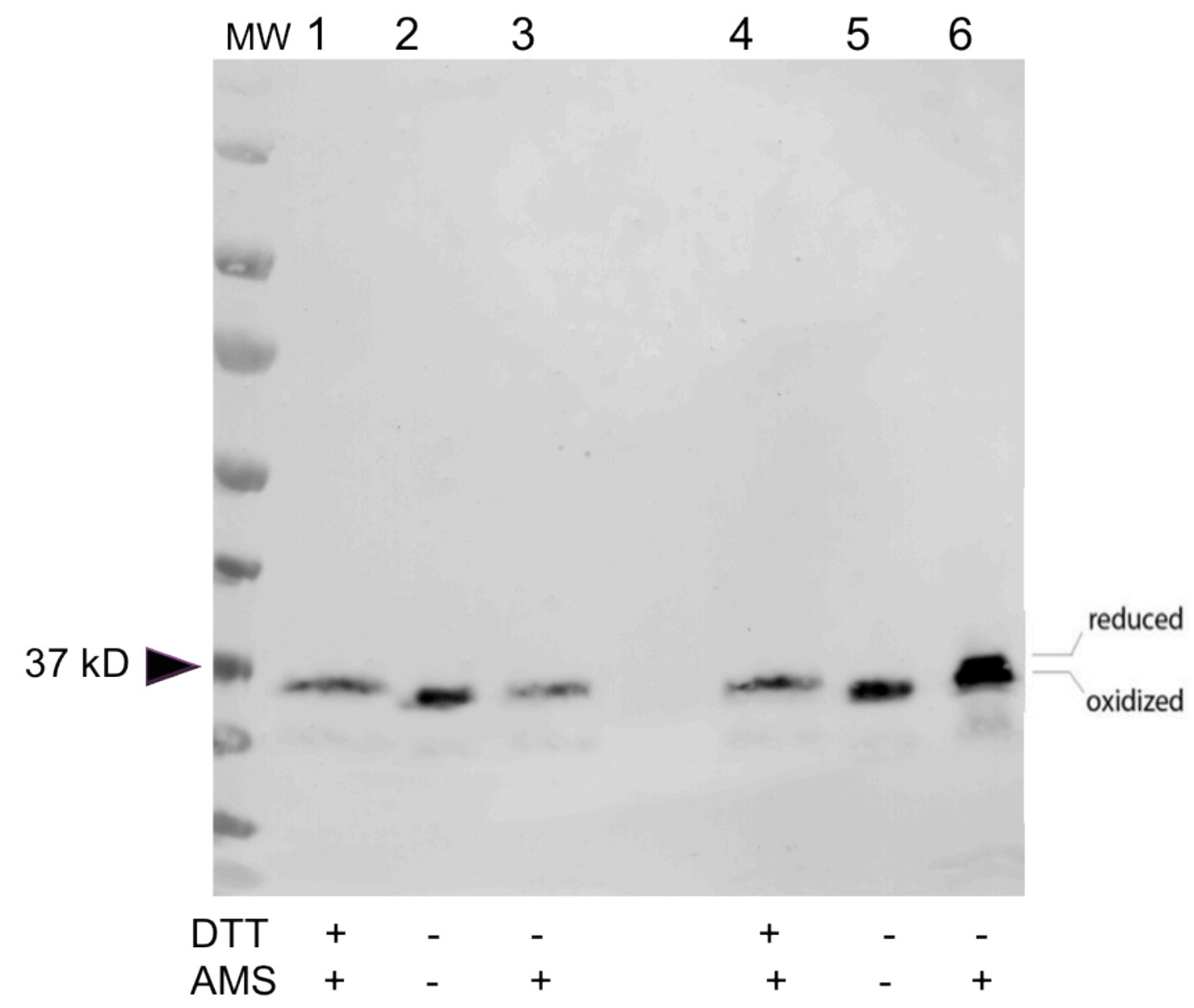

Figure 20. Redox status of Lpn DsbA2 in E. coli as determined by AMS-alkylation. After TCA precipitation of whole cells, AMS alkylation adds $\sim 495$ Da to free cysteine residues, which can then be visualized as a shift in apparent molecular weight. Lanes $1 \& 4$ correspond to the fully reduced and alkylated migration of Lpn DsbA2. Lanes $2 \& 5$ correspond to the oxidized control, DsbA2 without AMS alkylation. Lanes 3 \& 6 AMS-alkylated whole cells, and in (lane 6), DsbA2 redox status in $E$. coli showed that DsbA2 exists as a mixture of oxidized and reduced forms in the presence of Lpn $d s b$ genes during stationary phase. 


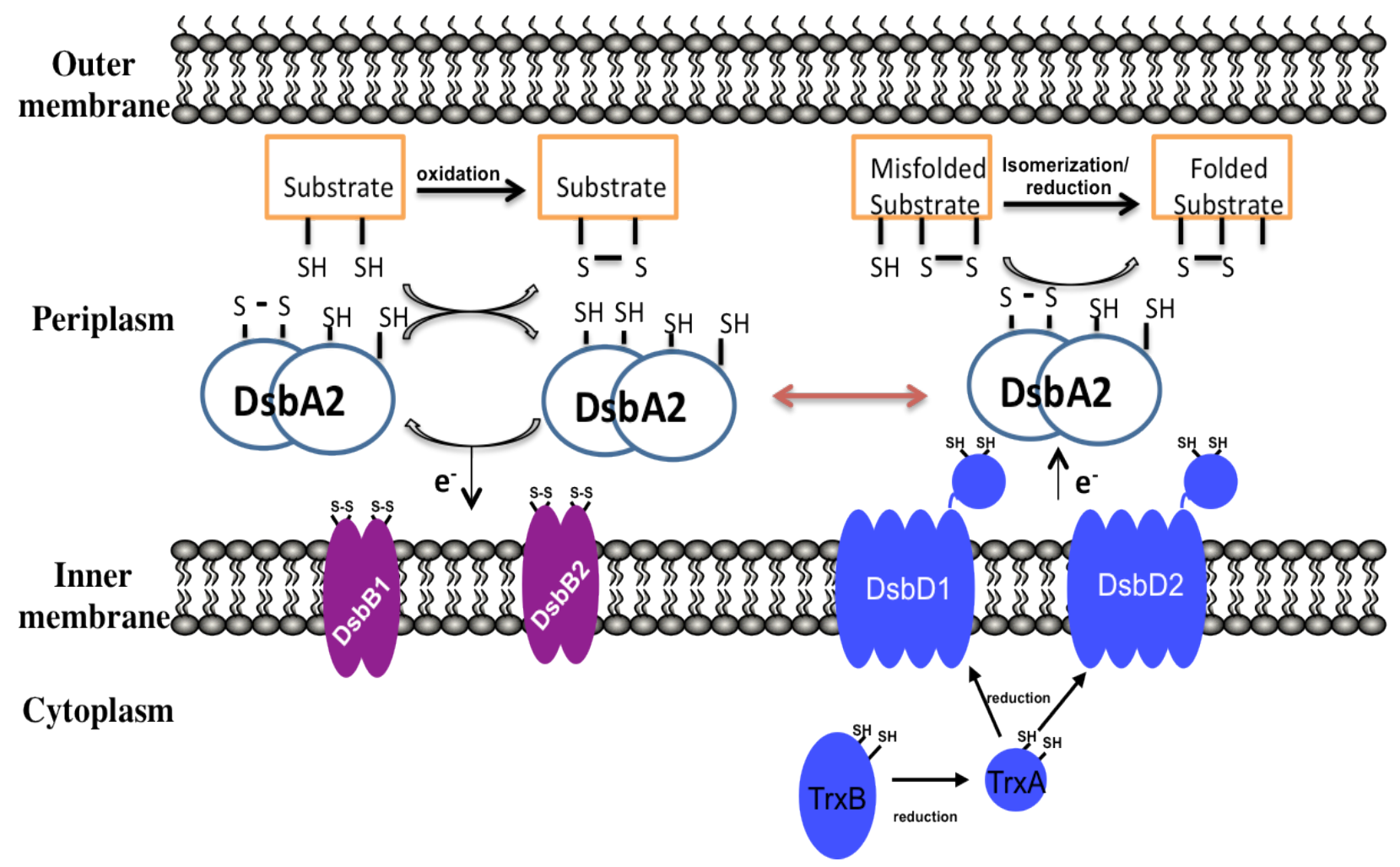

Figure 21. Disulfide bond trafficking in $L$. pneumophila. DsbA2 catalyzes the formation of consecutive disulfide bonds in reduce substrates in the periplasmic. Either DsbB1 or DsbB2 to repeat the oxidation cycle reoxidizes DsbA2. However, if misoxidation occurs in substrates requiring non-consecutive disulfide bonds, DsbA2 also contributes the isomerase activity; reduces and correctly oxidizes disulfide bridges and is kept in a reduced state by either DsbD1 or DsbD2. 
player DSB pathway that appears most often in slow-growing, pathogenic bacteria such as Lpn (Fig. 21).

\subsection{DsbA2 mutant constructs have no growth or expression defect in vitro}

The pathogenicity of L. pneumophila is highly dependent on the production of virulence factors such as type IV secretion system (T4SS). In addition to L. pneumophila, many slowgrowing water-borne and soil bacteria as well as vector-borne pathogenic bacteria containing the T4SS also contain DsbA2-like protein as described in (Kpadeh et al., 2013). Here, we show for the first time DsbA2 is a bifunctional enzyme in Lpn. In order to investigate if this system has an advantage in slow-growing bacteria, we utilized E. coli (WT) and RGP438 constructs containing pMMB206 empty vector, ZK01::pA2B1, ZK01::pA2B2, and ZK02::pA2B2 in in vitro growth assay for any defect due to the reconstitution of $d s b$ genes in $E$. coli. In vitro growth characteristic in LB broth with 0 and $1 \mathrm{mM}$ IPTG were monitored overtime. As seen in Fig. 22, expression of these mutant proteins resulted in a distinguishable defect of bacterial growth compared to the empty vector control and uninduced controls. These studies are consistent with the hypothesis that expression of L. pneumophila dsb genes in E. coli has a measurable effect on in vitro growth, suggesting that the DsbA2 system might be favored in slow growing soil and aquatic bacteria.

\subsection{Monomeric DsbA2 mutant exhibits a defect in attachment, invasion and intracellular replication}

For intracellular pathogens, such as L. pneumophila, the ability to attach, invade, and multiply within host cells is pivotal for virulence. We recently reported that the extracytoplasmic assembly of the T4SS of L. pneumophila is dependent on correct disulfide bond formation 
(A) - IPTG

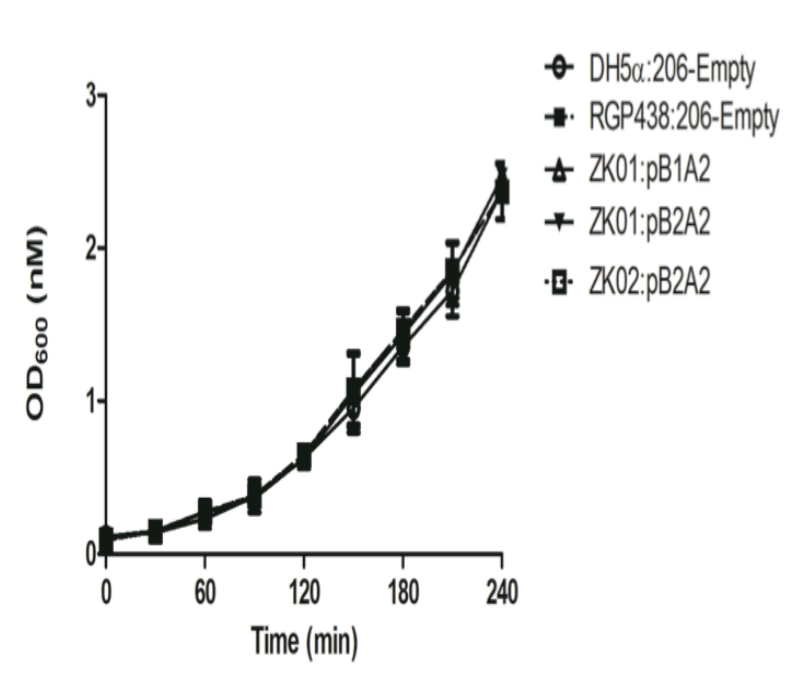

(B) + IPTG

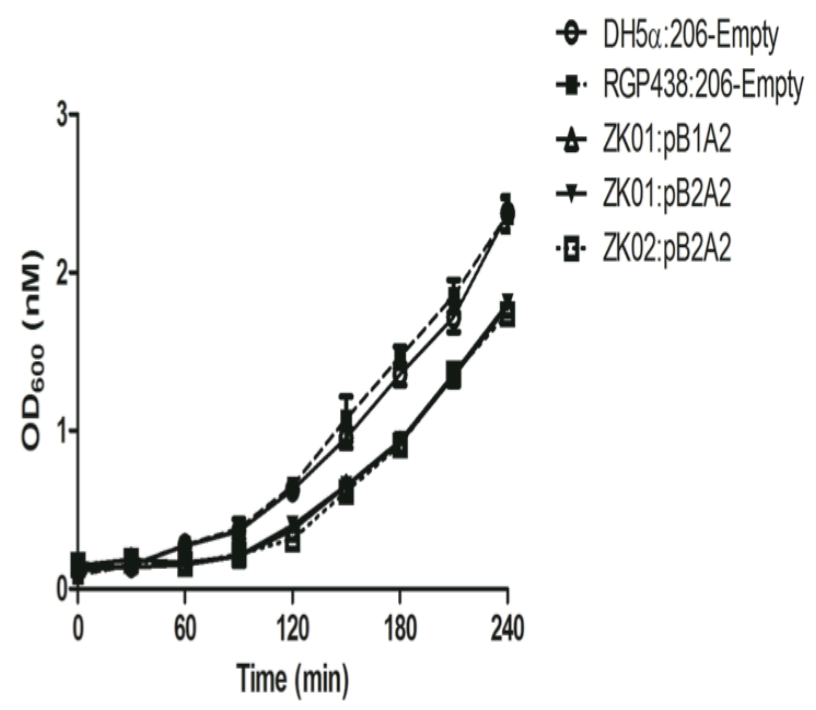

Figure 22. Growth assay. In vitro growth assessment of E. coli containing L. pneumophila dsb genes before and after induction with $1 \mathrm{mM}$ IPTG. Overnight grown bacteria were resuspended in fresh medium to an OD $\sim 0.05$, incubated for $30 \mathrm{~min}$, and then induced at OD $\sim 0.2$ with $1 \mathrm{mM}$ IPTG. Bacteria growths were assessed at each time point as indicated. 
catalyzed by a novel and essential disulfide bond oxidoreductase DsbA2 and not by DsbA1, a second dispensable DSB (Jameson-Lee et al., 2011).

To assess whether expression of monomeric DsbA2 is important for virulence as compared to reduced DsbA2, DsbA2 $\Delta \mathrm{N}$ was used as a tool to assess the effect of oxidized DsbA2 activity in vivo. We created a P198T mutant in both monomeric and dimeric proteins in $L$. pneumophila strain AA100, a strategy adapted from (Jameson-Lee et al., 2011) to test whether expression of mutant proteins might affect periplasmic funtions related to virulence. Expression of constructs was pre-induced with $1 \mathrm{mM}$ IPTG. We first assessed the role of the mutant DsbA2 proteins in replication inside amoeba, a natural host of Lpn. Expression of DsbA2 $\Delta \mathrm{N}$ P198T exhibited a defect in intracellular replication in amoeba as compared to AA100 WT (Fig. 23). However, since amoebae are naturally phagocytic, it is difficult to conclude whether the dominant-negative effect on intracellular growth by DsbA2 $\Delta \mathrm{N}$ P198T was due to defects in cellular attachment, invasion, and/or replication. In order to examine these possibilities we utilized a model of Lpn invasion of non-phagocytic HeLa cells (Garduno et al., 1998c; McCusker et al., 1991; Newton et al., 2008; Jameson-Lee et al., 2011). As depicted in Figure 24, L. pneumophila strain AA100 pMMB206:empty vector (positive control) under inducing conditions readily attached and invaded HeLa cells, while DsbA2 $\Delta \mathrm{N}$ P198T expressing mutant exhibited a three fold decrease in internalization nearly, similar to dotA mutant (negative control) and the DsbA2 P198T mutant previously shown to be dominant negative (Jameson-Lee et al., 2011). Note that $\operatorname{dot} A$, which has a defect in Dot/Icm activity is able to attach to HeLa cells but unable to invade after PBS wash. These results indicate that overexpression of monomeric DsbA2 P198T mutant protein interferes with DsbA2 function in the periplasm; thereby resulting 

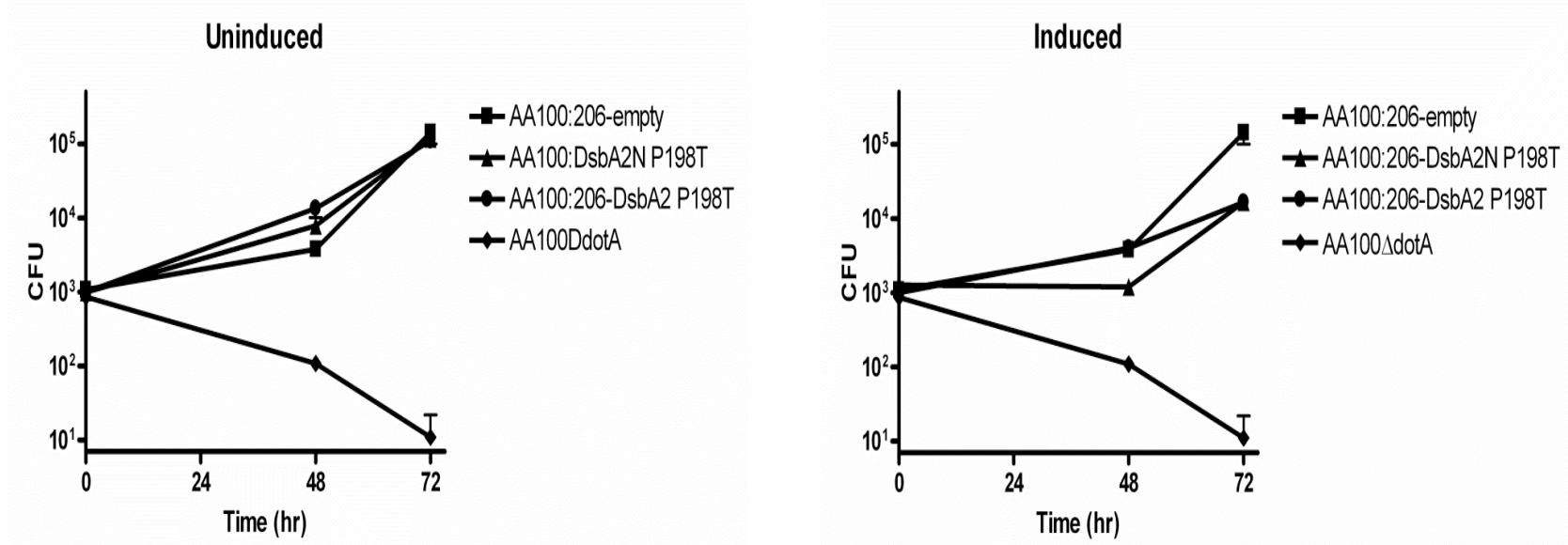

Figure 23. Intracellular replication in amoeba. Acanthamoeba castellanii were infected with $L$. pneumophila AA100 constructs (uninduced) in the absence of IPTG induction, (induced) induction at $1 \mathrm{mM}$ IPTG. Colony-forming units (cfu) were determined in triplicate at the indicated times, and a representative assay is presented. Expressions of P198T mutant proteins inhibit intracellular growth in amoeba. DsbA2N represents DsbA2 $\Delta N$. Statistical significance of $\leq 0.043$ by Student's t-test at $72 \mathrm{~h}$. Experiment performed in conjunction with Shandra Day, MD. 


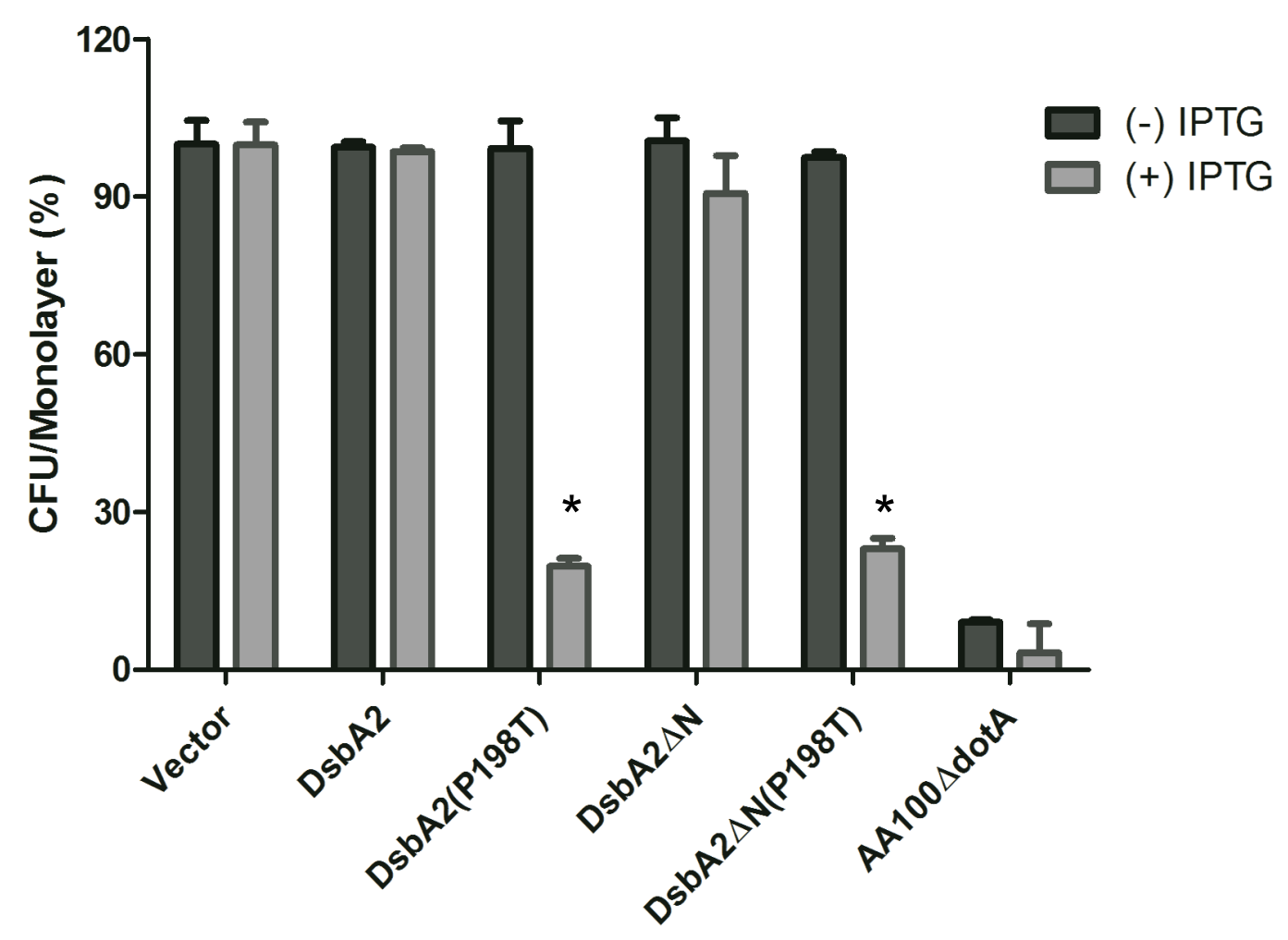

Figure 24. Attachment and invasion of non-phagocytic HeLa cells by Lpn expressing wild-type and mutant DsbA2 constructs. HeLa cells were infected with L. pneumophila AA100 constructs: AA100 WT pMMB206-empty vector (positive control), pMMB:dsbA2 P198T and $\operatorname{dot} A$ (negative controls), pMMB:dsbA2, pMMB:DsbA2 $\Delta \mathrm{N}$ and pMMB:dsbA2 $2 \mathrm{~N}$ P198T in the absence (dark gray) or presence of $1 \mathrm{mM}$ IPTG (gray). Colony-forming units (cfu) were determined in triplicate at the indicated times for a single experiment; experiment was repeated multiple times. Over expression of DsbA2 $\mathrm{N}$ P198T protein in L. pneumophila exhibits a defect in attaching and invading HeLa cells. Asterisk $\left(^{*}\right)$ denotes statistical significance of $\leq 0.005$ by Student's $t$-test. Experiment performed in conjunction with Shandra Day, MD. 
in a dominant-negative effect on attachment, invasion, and intracellular multiplication, indicating a role for DsbA2 function in L. pneumophila pathogenesis.

\subsection{Functional Dot/Icm T4SS assessment of monomeric DsbA2 activity}

Since a functional Dot/Icm T4SS (T4SS) is essential for invasion and intracellular multiplication of L. pneumophila in host cells (Berger and Isberg, 1993; Brand et al., 1994; Franco et al., 2009; Jameson-Lee et al., 2011), we considered it likely that the dominant-negative effect of the DsbA2 $\Delta$ N P198T mutant protein was due to interference on DsbA2 bifunctional activity, thus affecting with either assembly or function of the T4SS secretion apparatus. To directly address this possibility, we employed a well-established contact hemolytic assay that was previously validated with specific Dot/Icm T4SS $(\Delta$ dotA) and dimeric DsbA2 P198T mutants (Kirby et al., 1998; Charpentier et al., 2009; Jameson-Lee et al., 2011). As seen in Figure 25, AA100 pMMB206:empty vector mediated lysis of erythrocytes in the presence or absence of IPTG. In contrast, bacteria expressing the dominant-negative DsbA2 $\Delta$ N P198T mutant protein did not exhibit contact-dependent hemolysis similar to DsbA2 P198T (induced), and nearly equivalent to that of a dotA mutant (negative control), respectively. Here, expressed DsbA2 $\Delta \mathrm{N}$ construct also show a slight defect in hemolysis but was not significant compared to wild-type AA100 strain. Longer incubations also showed no change in erythrocyte lysis by bacteria expressing either DsbA2 $\triangle \mathrm{N}$ P198T or DsbA2N P198T. These results further validate the correlation of defects seen in HeLa cells infectivity assay, suggesting that the dominant-negative avirulence phenotype of the DsbA2 $\Delta \mathrm{N}$ P198T mutant protein most likely results from interference on DsbA2 optimal function in the periplasm, leading to the disruption of assembly or function of the Dot/Icm T4SS. 


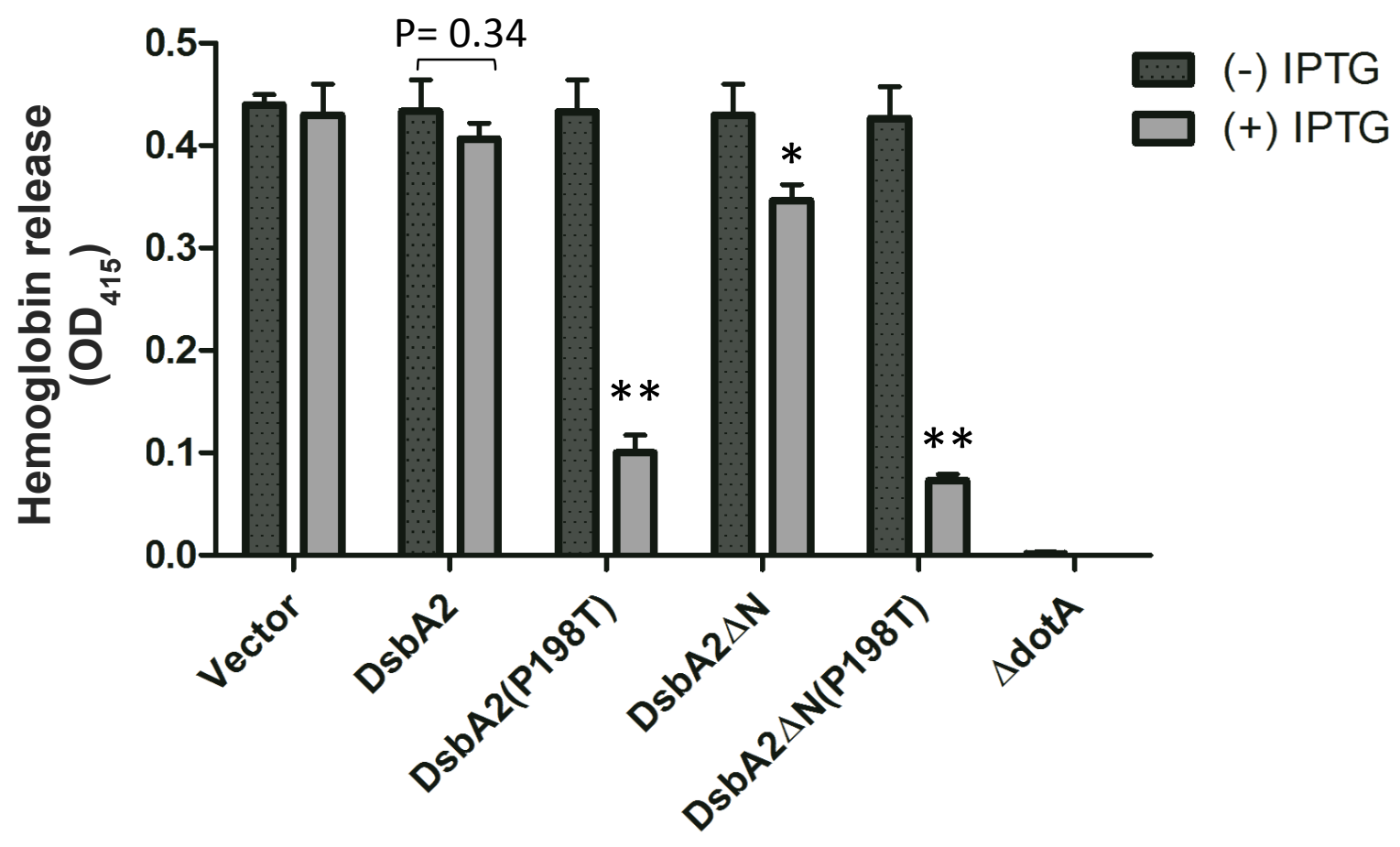

Figure 25. Effects of DsbA2 wild-type and mutant constructs on Dot/lcm T4SS-mediated erythrocyte lysis. Red blood cells were infected with L. pneumophila AA100 constructs in the absence of IPTG induction (dark gray), and induction at 1mM IPTG (gray). Hemoglobin release was measured after $2 \mathrm{~h}$ incubation of erythrocytes with AA100 WT pMMB206-empty vector (positive control), pMMB:dsbA2 P198T and $\operatorname{dotA}$ (negative controls), pMMB:dsbA2,

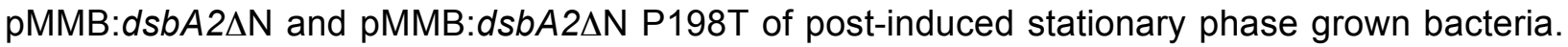
Asterisks $\left({ }^{*}\right),\left({ }^{* *}\right)$ denote statistical significance of $\leq 0.0432$ and $\leq 0.001$ by Student's $t$-test, respectively. Experiment performed in conjunction with Shandra Day, MD. 


\subsection{The importance of DsbA1 for $L$. pneumophila pathogenesis}

Proteins that contain disulfide bonds are often slow to fold in vitro because the oxidation and correct pairing of the cysteine residues is rate limiting. The folding of such proteins is greatly accelerated by a periplasmic oxidoreductase DsbA, but the mechanism of this rate enhancement is not well understood. L. pneumophila contains two oxidoreductase proteins; DsbA1 and DsbA2. DsbA1 has been shown to be non-essential and without phenotype (Jameson-Lee, UVa thesis 2012) when compared to DsbA2, which has been characterized in detailed above. In addition to further characterizing the role of DsbA2 for Lpn pathogenesis, our study here shows that a $d s b A 1$ knockout mutant is defective in pigment production (Fig. 28) and demonstrates a hypervirulent phenotype in an L929 cell infectivity assay (Fig. 29) and enhanced lysis of erythrocytes (Fig. 30A). The mutant also retained the ability to replicate in amoeba (data not shown), and to attach and invade HeLa cells (Fig. 30B). We also determined the crystal structure of Lpn DsbA1, and found that its structure closely resembles the ubiquitous redox protein thioredoxin and E. coli DsbA, despite very low sequence similarity. An important similarity to E. coli DsbA is the presence of another domain that forms a cap over the thioredoxin-like active site of DsbA1; however, this differs when compared to E. coli DsbC which is a dimer. These features suggest that DsbA1 may compete for substrates with DsbA2 or might act by binding to partially folded polypeptide chains before oxidation of cysteine residues like E.coli DsbA. Also, its hypervirulent phenotype is indicative of its modulating role on Lpn virulence. We hypothesized that DsbA1 functional and structural similarities to E. coli DsbA suggest a contributing role to the virulence of L. pneumophila in its host. 
A.

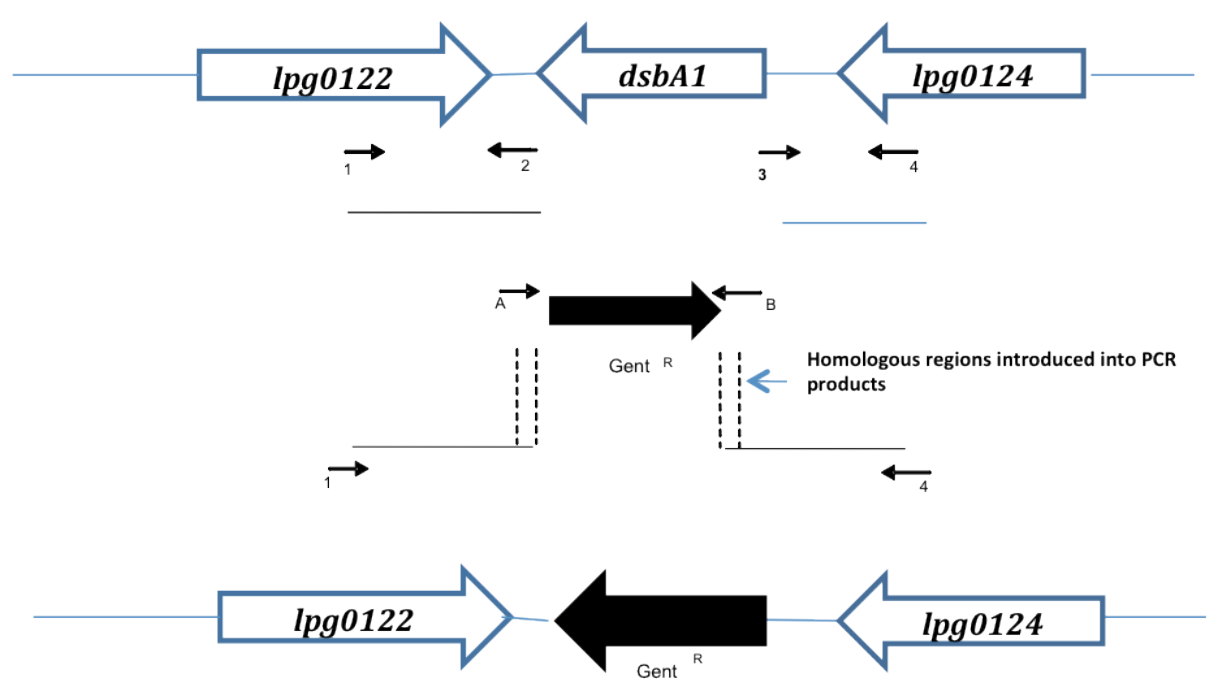

B.
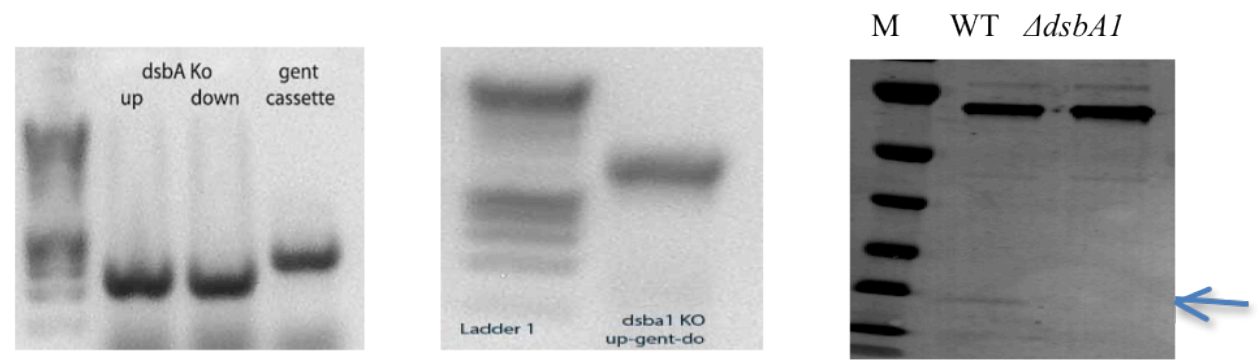

Figure 26. dsbA1 knockout construction. A) Genetic organization of the $d s b A 1$ locus and allelic replacement strategy to introduce a gentamicin cassette downstream of dsbA1. Vectorfree allelic replacement. Primers (arrows) amplify $\sim 500 \mathrm{bp}$ chromosomal regions (lines) surrounding Lpn dsbA1. Primers were used to amplify an antibiotic resistance cassette $\left(G^{2} t^{R}\right)$. In a separate reaction, the two flanking regions and cassette were amplified with the two outside primers (1\&4). The dashed lines denote homologous regions introduced into the PCR products where they can anneal to form one large template (B; middle diagram). The final PCR product contains a resistance cassette flanked by chromosomal regions to facilitate homologous recombination. B) Last diagram - $\alpha$-DsbA1 antibody was used to confirm $d s b A 1$ knockout. The blue arrow points to the $24 \mathrm{kDa}$ DsbA1 protiein. 
A. $\alpha$-DsbA1

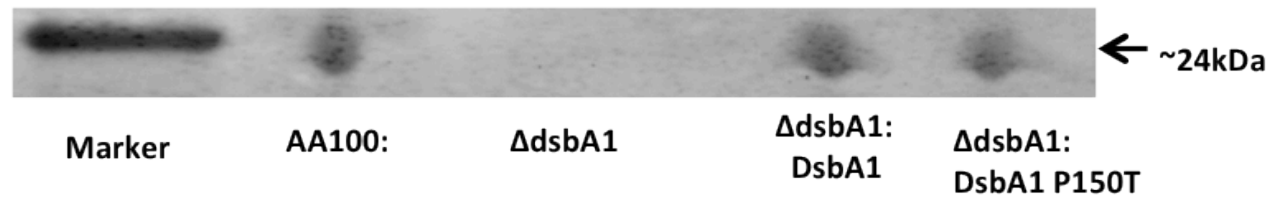

B. Extracellular growth of Legionella pneumophila

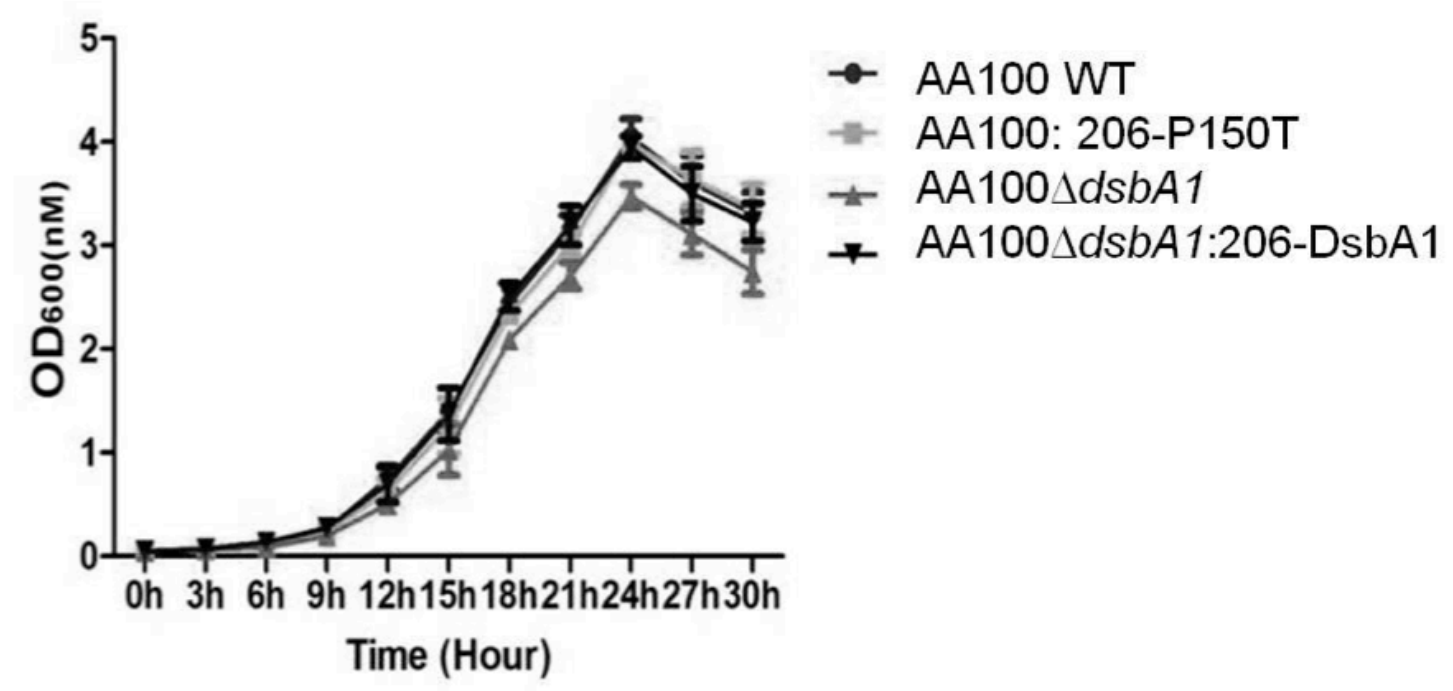

Figure 27. Protein expression and in vitro growth assessments of WT and dsbA1 mutant Lpn. (A) Proteins expressions of all construct using $1 \mathrm{mM}$ IPTG at stationary phase. Western blot analysis with DsbA1-specific antibody validated a successful deletion of $d s b A 1$. (B) Growth curve of L. pneumophila constructs show no significant difference 


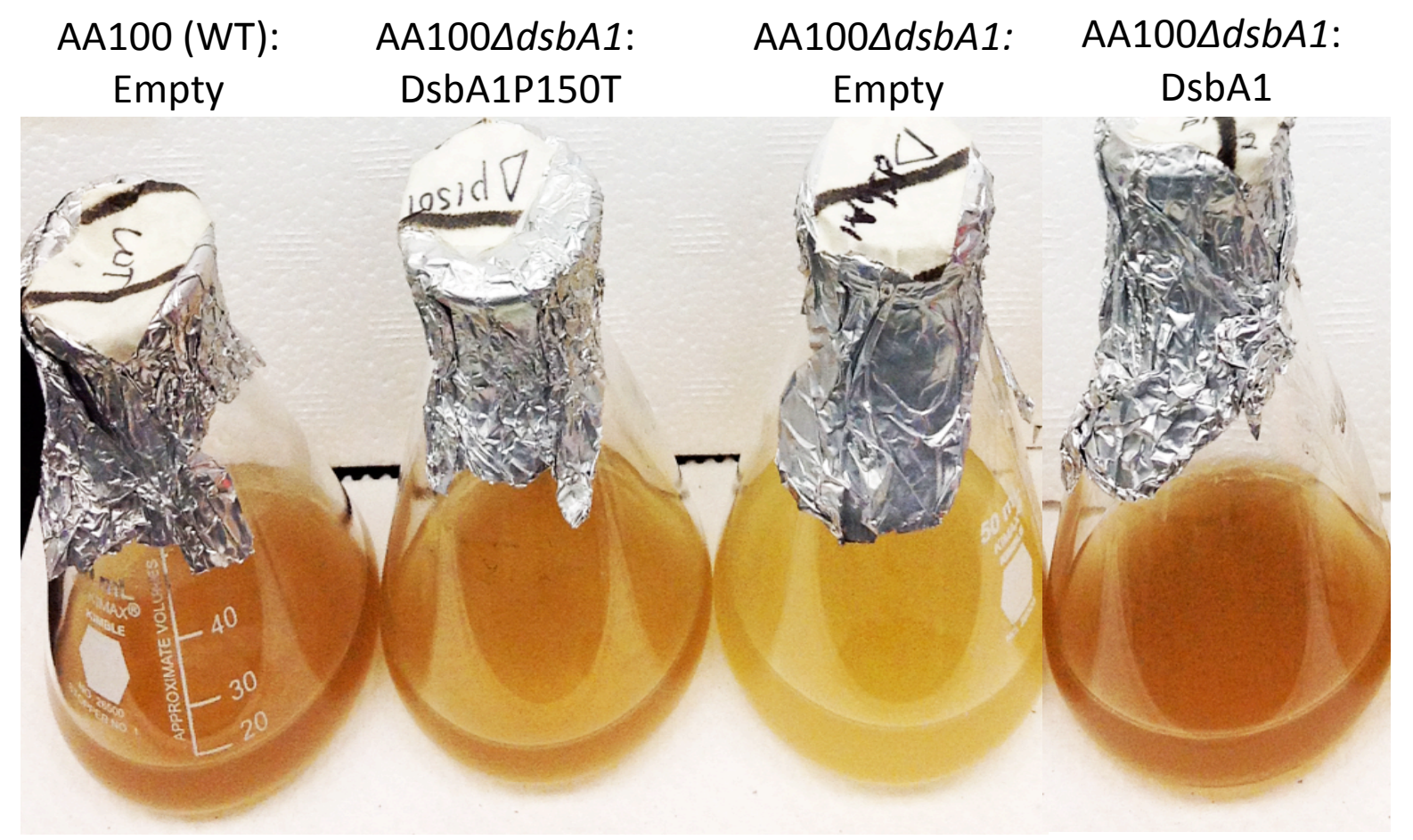

Figure 28. L. pneumophila DsbA1 has a defect in pigment production. Overnight culture of L. pneumophila WT strain AA100 (pMMB206::empty vector), AA100 $\Delta d s b A 1$ (pMMB206::empty vector), pMMB206::dsbA1 P150T, and pMMB206::dsbA1 were grown to $\mathrm{OD}_{600} \sim 0.6$, then induced with $1 \mathrm{mM}$ IPTG and grown till 30h (stationary phase). AA100 $\Delta d s b A 1$ exhibited a defect in pigment production when compared to AA100 WT and $\Delta d s b A 1$ complemented with expression of DsbA1 in an inducible vector (pMMB206::dsbA1). 


\subsection{Novel phenotype: $d s b A 1$ knockout exhibits a defect in pigment production}

Prior to this study, DsbA1 has been reported to be dispensable for virulence in $L$. pneumophila (Jameson-Lee, UVa thesis, 2012). We sought to closely assess the growth differences of Lpn AA100 strains with or without the $d s b A 1$ gene. Our results of overnight grown constructs in BYE broth reveal that DsbA1 knockout exhibits a loss in pigment production, as depicted in Fig. 28. However, the defect was restored upon expression of pMMB206:dsbA1 in a $d s b A 1$ knockout background. This result suggests that the reason $d s b A 1$ is not selected against in Lpn may be due to its ability to modulate virulence both during exponential growth where DsbA1 and DsbA2 levels are low and to a lesser extent during stationary phase when $d s b A 2$ expression is four fold higher than that for DsbA1. In head to head growth studies in vitro, there was no significant difference between the WT AA100 and the $d s b A 1$ mutant (Fig. 27).

\section{$3.18 d s b A 1$ knockout exhibits a hypervirulent phenotype in plaque assay}

Plaque assay is a widely used approach for determining the quantity of infectious bacteria/virus. This technique was first developed to calculate the titers of bacteriophage stocks. Renato Dulbecco modified this procedure in 1952 for use in animal virology, and it has since been used for reliable determination of the titers of many different viruses, which has been adopted by bacteriologists.

We constructed a $\triangle d s b A 1$ mutant using a vector-free allelic replacement mutagenesis strategy (Fig. 26). We performed plaque assay using Legionella AA100 strain containing our $\triangle d s b A 1$ mutant and complements containing (pMMB206:empty vector, pMMB206:dsbA1-P150T, and pMMB206:dsbA1), 10-fold dilutions of overnight grown bacterial stocks were prepared, and 
A.

Control $1.5 \times 10^{\circ}$ L929 Cells only

AA100:pMMB206Empty
$\mathrm{AA} 100 \Delta d s b A 1:$ pMMB206-Empty
$\mathrm{AA} 100 \Delta d s b A 1:$ pMMB206-DsbA1
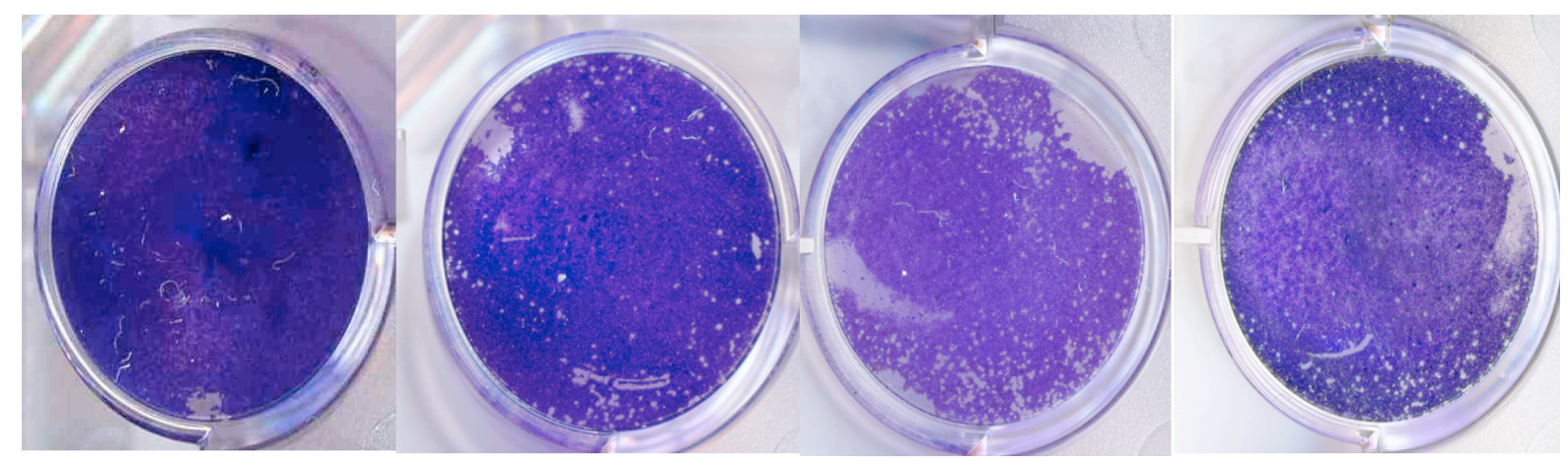

B.

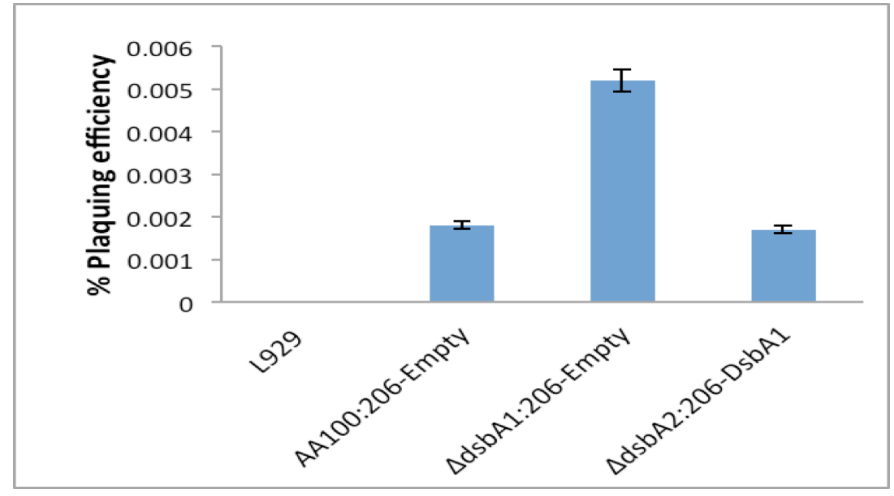

Figure 29. Lpn DsbA1 knockout exhibits a hypervirulent phenotype in L929 infectivity assay. (A) Crystal violet which stains live cells. Zone of clearance is indication of plaques forms due to the lysis of L929 cells by virulent bacteria. (B) Plaquing efficiency of Lpn DsbA1 knockout exhibits a hypervirulent phenotype compared to L. pneumophila WT AA100 strain (positive control), and L929 cells without any bacteria (negative control). $\Delta \operatorname{dot} A$ mutant displayed no plaquing efficiency similar to L929 negative control (data not shown). Experiment performed in conjunction with Shandra Day, MD. 
$0.2 \mathrm{ml}$ aliquots are inoculated onto susceptible L929 cell monolayers in 1.8ml DMEM in a 24 well plate, as described in methods . After an hour incubation period, to allow bacteria to attach to cells, DMEM containing the unattached bacteria are removed, and the monolayers were covered with DMEM plus $0.6 \%$ agarose (SeaKem) gel pad. The plates were incubated for four days. The original infected cells released progeny bacteria, and the spread of the new bacteria was restricted to neighboring cells by the gel. Consequently, each infectious particle produces a circular zone of infected cells called a plaque. Eventually the plaque becomes large enough to be visible to the naked eye. Dyes that stain living cells, such as cystal violet, which is used here, are often used to enhance the contrast between the living cells and the plaques. Only bacteria/virues that cause visible damage of cells can be assayed in this way. As depicted in Fig. 29, our results revealed L929 cells infected with DsbA1 knockout strain showed an increase in plaquing compare to AA100 WT strain. Also, overexpression of DsbA1 from an inducible vector was able to restore the plaquing efficiency to WT levels. These results suggest the novel role of DsbA1 as a modulator in the system for L. pneumophila pathogenesis.

\subsection{DsbA1 is a modulator of virulence in Legionella pneumophila}

In order to validate the modulating role of DsbA1 for pathogenesis in Legionella, we sought to utilize in vivo infectivity assays to demonstrate the contributing role of DsbA in the DSB system using $\triangle d s b A l$ mutant. As reported in Fig. $30 \mathrm{~A}, \triangle d s b A l$ mutant bacteria were very efficient in lysing RBC as depicted by the increased in hemoglobin release as compared to AA100 WT. Overexpression of WT DsbA1 in a $\triangle d s b A 1$ mutant background reversed the hypervirulent phenotype to wild type level. Next, we tested the ability of $\Delta d s b A 1$ mutant to attach and invade non-phagocytic HeLa cells. HeLa cells as previously described are commonly used 
A.

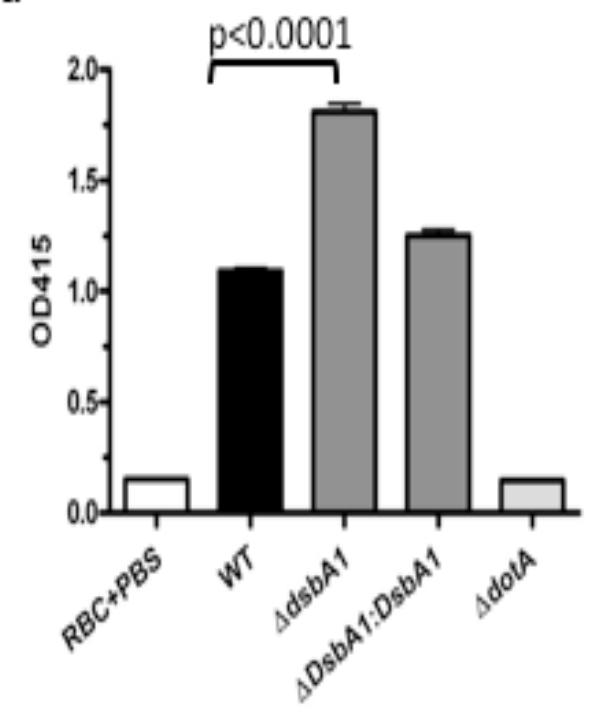

B.

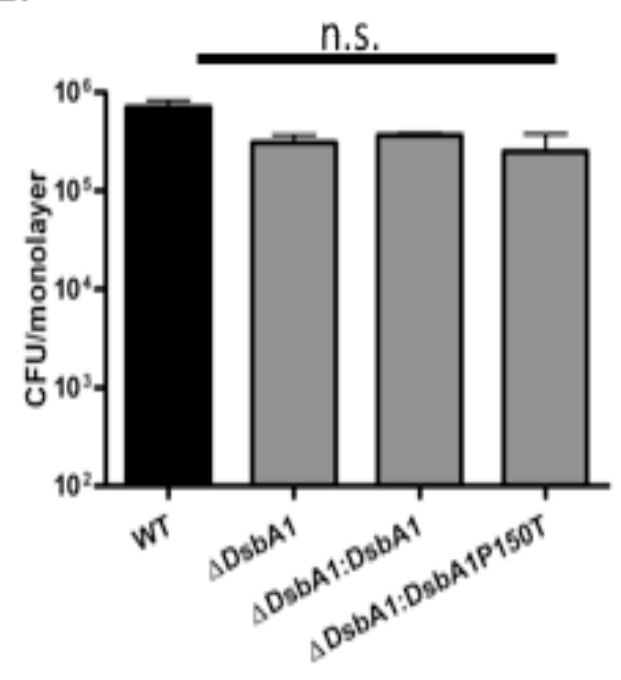

Figure 30. In vivo assays demonstrating the role of DsbA1 in L. pneumophila. (A) Functional Dot/lcm T4SS assessment was examined utilizing contact-dependent lysis of human RBC. Hemoglobin release was measured after $2 \mathrm{~h}$ incubation of erythrocytes with AA100 WT (positive control), dsbA1 KO, $\Delta d s b A 1: p M M B 206-d s b A 1, \Delta d s b A 1: p M M B 206-d s b A 1$ P150T, PBS and $\operatorname{dot} A$ (negative controls) of post-induced stationary phase grown bacteria. (B) Attachment and invasion of non-phagocytic cell. DsbA1 KO has no defect in attaching and invading HeLa cells. Experiment performed in conjunction with Shandra Day, MD. 
to test the invasiveness of many bacterial species. The studies below aim to address the infectivity- attachment and invasion capabilities of DsbA1 mutants (knockout and P150T) as compared to AA100 WT for the assay. Using a stationary phase grown $\Delta d s b A 1$ mutant expressing DsbA1 P150T mutant protein, which also has a defect in resolving its substrates (as shown below) and DsbA1 WT in a $\triangle d s b A l$ background, we showed that there was no measureable defect in attachment and invasion of HeLa cells (as seen in Fig. 30B). These results reveal that while DsbA1 has an attenuating effect on virulence, it is not sufficient to have a dominant negative effect on DsbA2 funciton.

\subsection{DsbA1 interacts with substrates}

DsbA, a member of the thioredoxin superfamily, introduces disulfide bonds into newly translocated proteins. We sought to build on previous studies in which a His 6 -DsbA2 P198T mutant protein was used to capture and identify its substrate repertoire by using a cis-proline mutant of His $_{6}$-DsbA1 P150T constructed by site directed mutagenesis strategy, then SDS-PAGE, immunoblot/silver stain, and 2-dimensional (2D) diagonal gel were performed to examine DsbA1 substrate repertoire. Fig. 31A, shows the silver stained gel of DsbA1 interacting proteins separated by reducing and non-reducing SDS-PAGE. Remarkably, we saw DsbA1 P150T mutant accumulated bands of different apparent molecular masses, suggesting that these bands represent DsbA1-substrate complexes; these bands disappeared when samples were treated with reducing agent before electrophoresis (Fig. 31A, lane 3). 


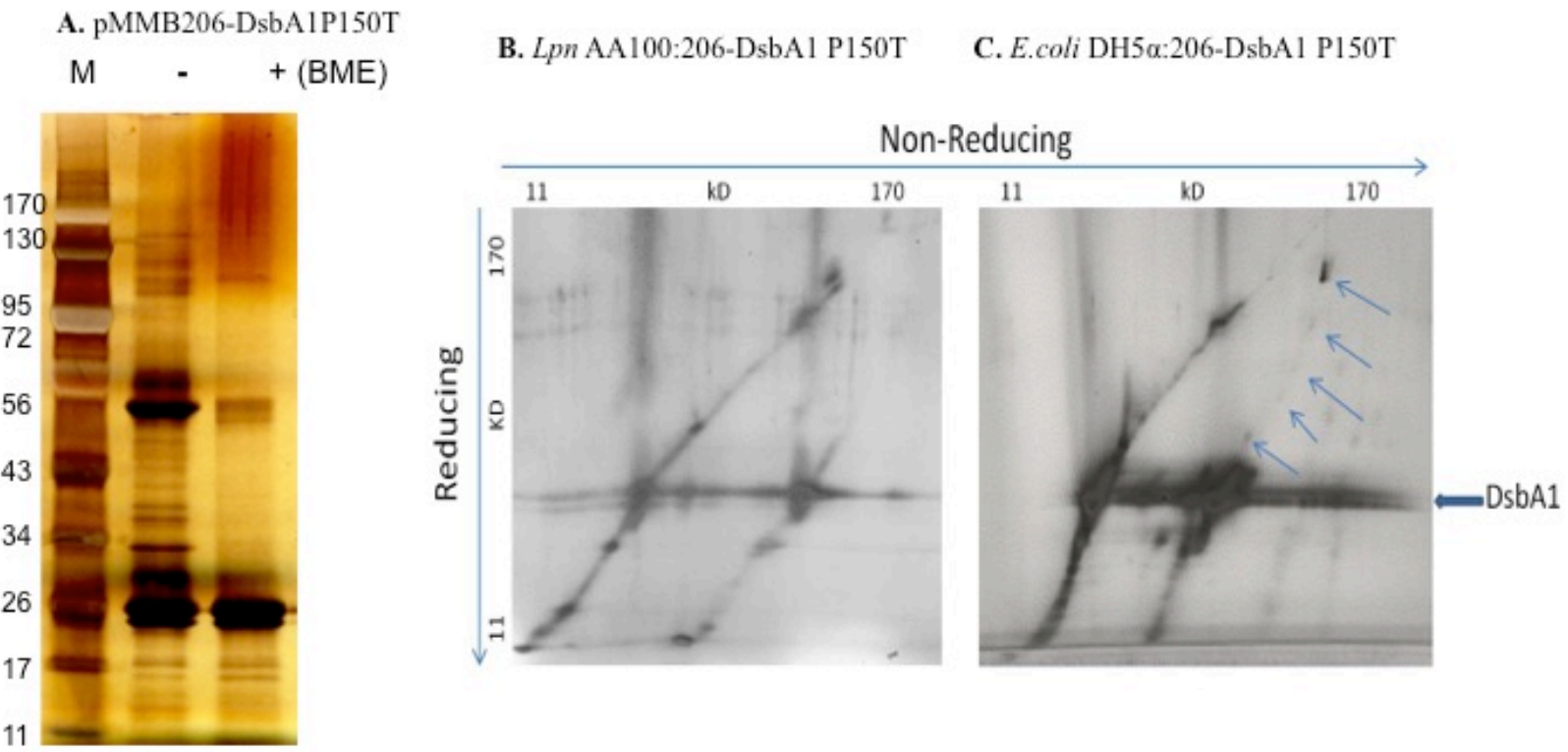

Figure 31. Substrate proteins capture by DsbA1 P150T mutant protein in L. pneumophila and E. coli. Legionella and E. coli strains containing C-terminal His-tagged DsbA1 P150T were induced in vitro. Substrate complexes were purified by nickel chromatography and diagonal gels of captured proteins were run under non-reducing (direction of electrophoresis indicate by arrows), then reducing conditions. Protein spots (silver stained) off the diagonal are DsbA1 interacting proteins whose disulfide bridge had been reduced in the second dimension. These proteins are yet to be identified by Mass-spectrometry (MS). (A) His 6 DsbA1-substrate complexes enriched for by $\mathrm{Ni}^{++}$column chromatography and separated by SDS-PAGE and then silver stained: lane 1 (MW marker), lane 2 (non-reducing conditions), lane 3 (under reducing conditions; note disappearance of high MW complexes), (B) In Legionella - DsbA1 P150T captured less periplasmic protein as compared to (C) E. coli, where the thinner arrows point to the difference in spots of substrate proteins that were captured. Thicker arrow points to DsbA1 that has dissociated from interacting proteins in the second dimension. 
To establish that the proteins were indeed captured, 2D diagonal gels with first dimension nonreducing and second dimension under reducing conditions was used (Fig. 31, B. Legionella, C. E. coli). Silver stained proteins spots off the diagonal represent DsbA1 captured proteins, which suggest that DsbA1 is an oxidase and interacts with substrates in the periplasm, and thus has a functional role in both L. pneumophila. Interestingly, overexpression of DsbA1 in E. coli shows an increased interaction with substrates than was noted for L. pneumophila. This finding suggests a moderate role/effect of DsbA1 for the proper folding of substrates in the periplasm of Lpn.

\subsection{Crystallization of L. pneumophila DsbA1}

The crystallization of DsbA1 was initiated by my predecessor (Jameson-Lee, UVA 2012). Lpn DsbA1 is predicted to have signal sequence peptides that cleaved between residues 20 and 21 (Signal P 4.0 online). We utilized the protocol of Missiakas et al. (1994); wherein periplasmic shockate of DsbA1 followed by anion exchange chromatography was used to generate a fulllength, un-tagged protein, and then sent to CSGID consortium for crystallization. Figures 32, 33, and 34 illustrate the similarities and differences between the crystal structures of the thioredoxin folds of Lpn DsbA1, EcDsbA, and EcDsbC, with the active site and N-terminus domain of Lpn DsbA1 being nearly similar to EcDsbA, but different from EcDsbC. Also, a crystallization study of Lpn DsbA2 using the same strategy is currently in progress. 

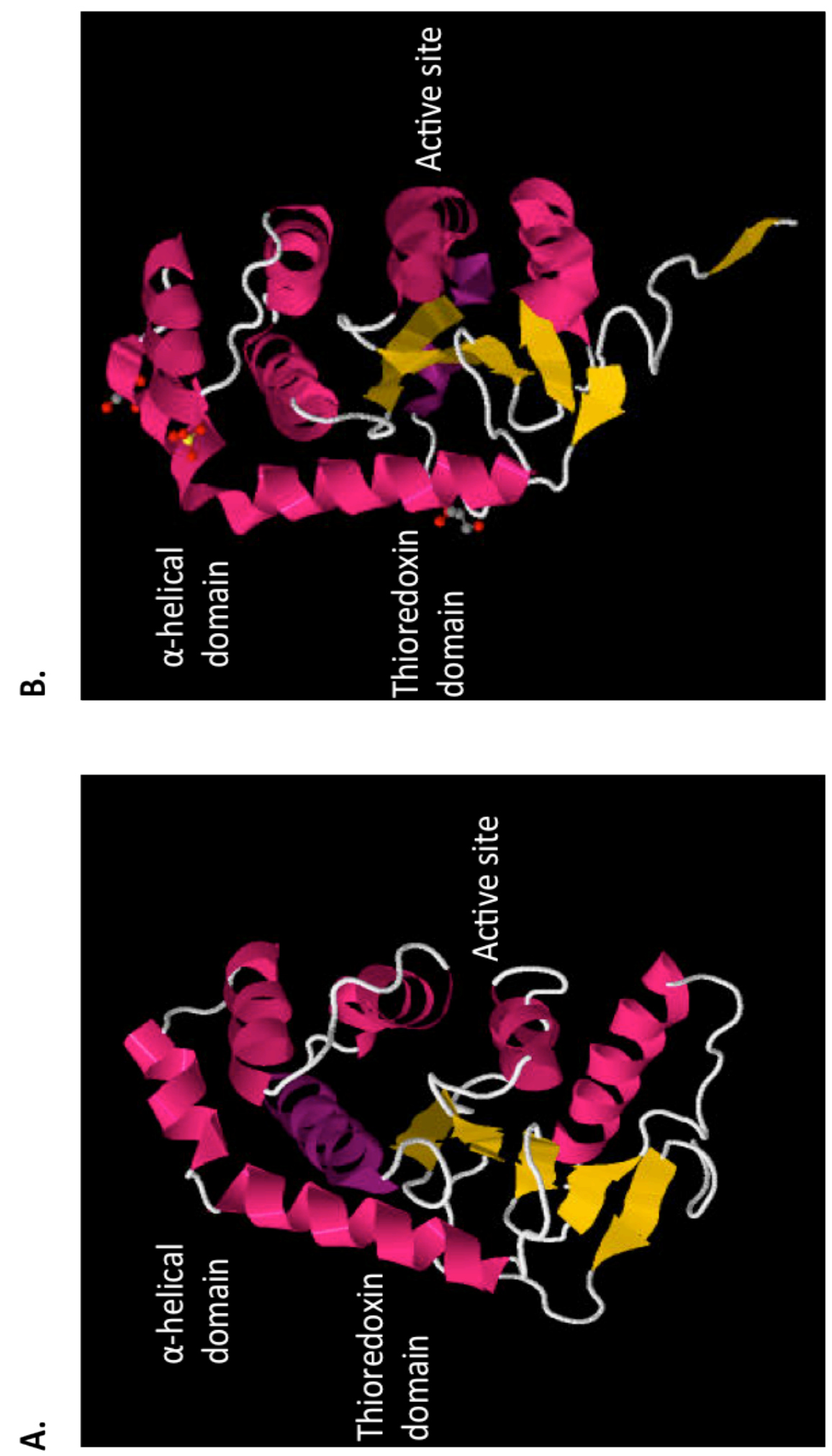
Figure 32. Crystal structures of EcDsbA and Lpn DsbA1. (A) EcDsbA (PDB 1a2L) and (B) Lpn DsbA1 (PDB 4jrr) depicting the active site consisting of the CXXC motif and cis-proline region. E. coli DsbA and $L p n$ DsbA1 thioredoxin folds have similar helices and conformations. 


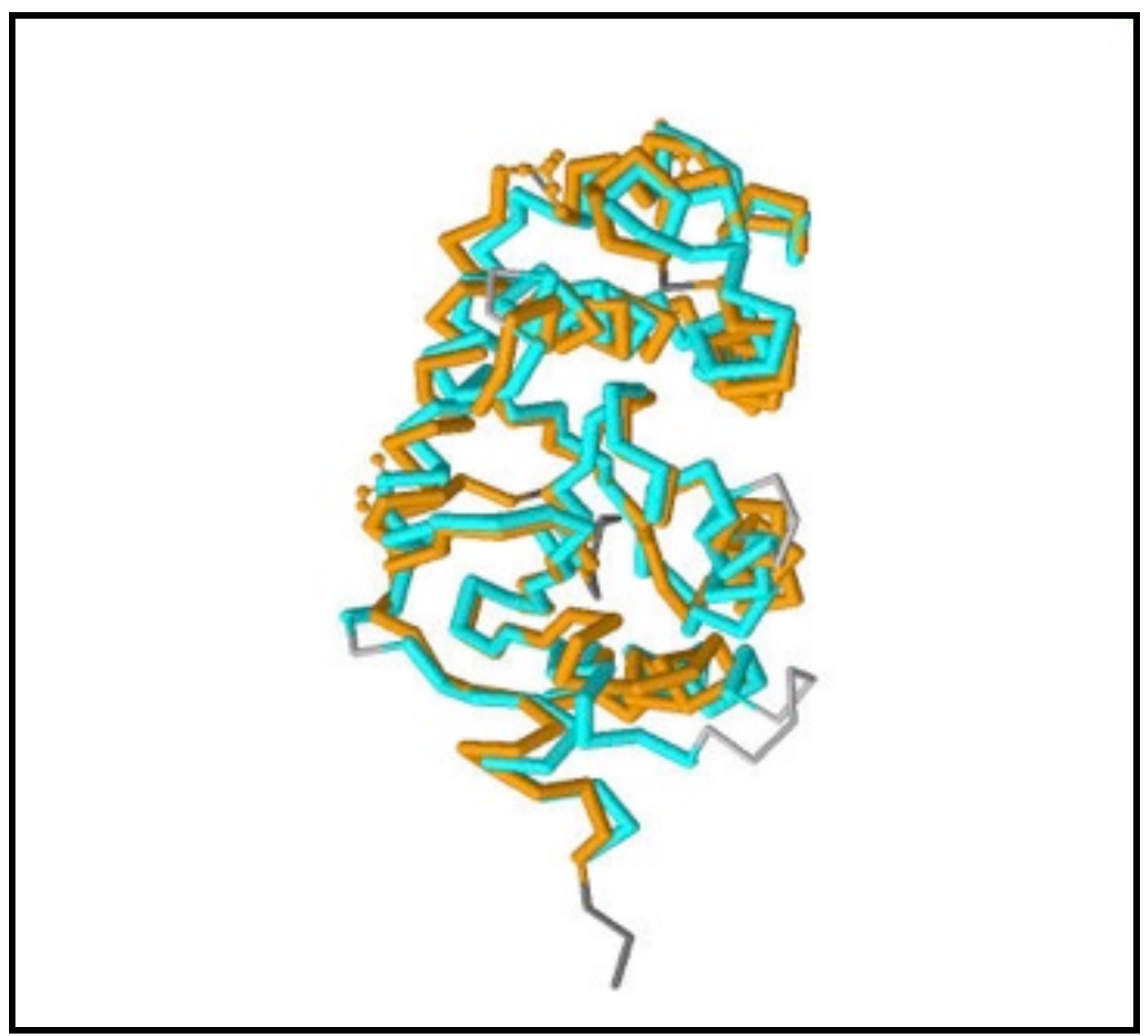

Figure 33. Superimposed. DsbA1 thioredoxin fold homologue crystallized from L. pneumophila is nearly identical to $E$. coli DsbA. Lpn DsbA1 (PDB 4jrr, orange) aligns well in space with EcDsbA (PDB 1a2j, blue). 
A.

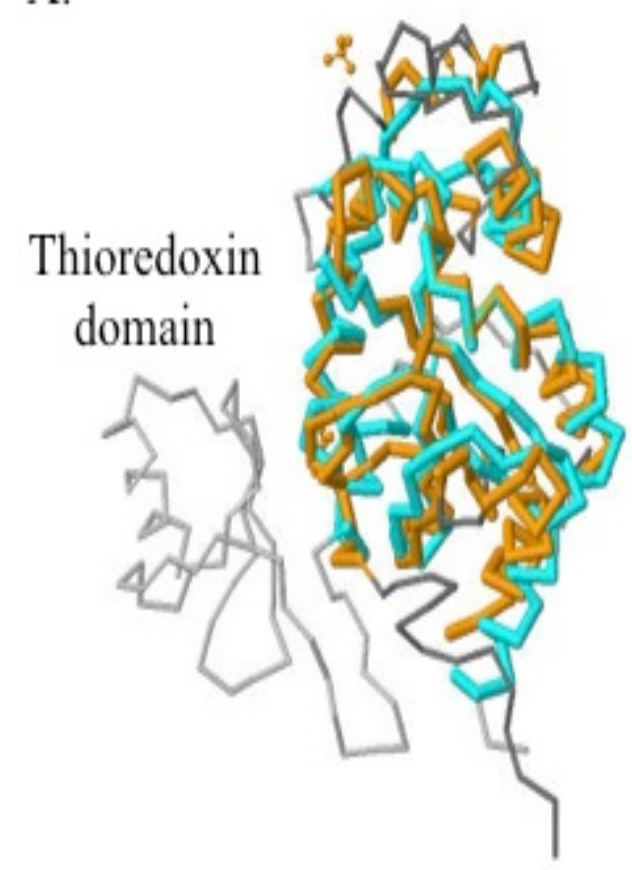

B.

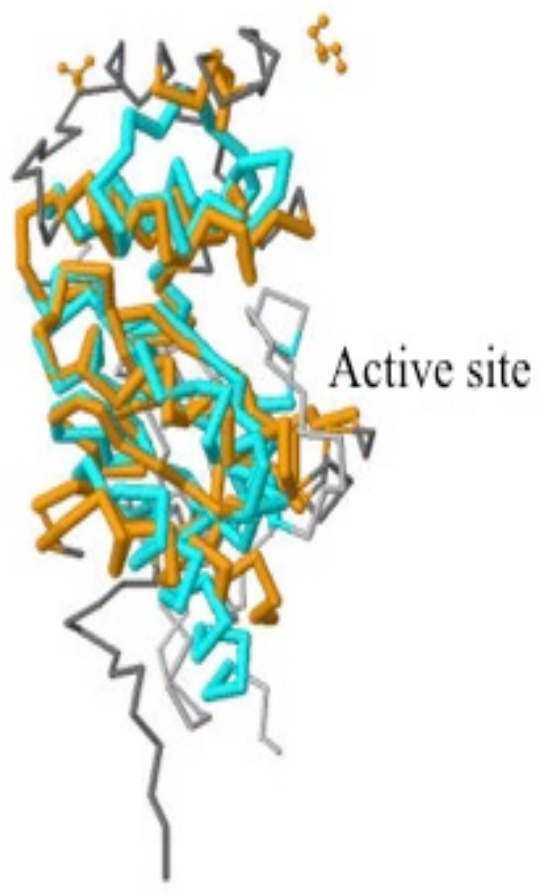

Figure 34. The thioredoxin fold and the active site of EcDsbC do not match up well with Lpn DsbA1. (A) E. coli DsbC (PDB 1eej, blue) contains extra alpha helices at its $\mathrm{N}$-terminus that do not line up well with L. pneumophila DsbA1 (PDB 4jrr, orange) crystal structure, (B) the active site of Lpn DsbA1 is pointed downward while EcDsbC active site is pointed upward. Both the thioredoxin fold and active site of Lpn DsbA1 are more similar to EcDsbA than EcDsbC. 


\section{Chapter 4. Discussion}

Over the past 20 years, E. coli has served as the primary model system for the study of disulfide bond formation in Gram-negative bacteria. However, this picture changed with the discovery of sets of enzymes that utilize the chemical properties of cysteines to form or reduce disulfide bonds in the periplasm of other bacterial species (Kadokura et al., 2003; Jameson-Lee et al, 2011; Kpadeh et al., 2013). The findings of this study build on the seminal work of Dr. Max Jameson-Lee who discovered DsbA2 and speculated that DsbA2 likely formed a novel system that similarly managed disulfide bond formation and protein folding in L. pneumophila. The studies presented herein provide solid evidence to support the hypothesis that the DsbA2 system differs fundamentally from the DsbA/DsbC system of $E$. coli. In the DsbA2 system, a single bifunctional homdimeric protein (DsbA2) catalyzes both the formation of and reductive isomerization of disulfides to promote protein folding. Extensive phylogenetic analysis reveals that the bifunctional DsbA2 system, first discovered in Lpn, is common to much of the microbial world that includes diverse species of Gram-negative soil and aquatic bacteria and all intracellular

pathogens that express a type IV secretion system. Perhaps the most significant finding of these studies is that the DSB system of E. coli can be functionally replaced by the DSB system of Lpn. Our work raises many new questions about the advantages and disadvantages of a one player (DsbA2) system versus two-player (DsbA/DsbC) system in the management of disulfide bond formation and rearrangement in microbial systems.

\subsection{Importance of DsbA2}

Remarkably, the newly discovered DsbA2 system appears to be quite common and includes many soil and aquatic species such as Caulobacter, Bradyrhizobium, Aeromonas, and 
Azospirillum and most human, animal, and plant pathogens that express T4SSs as major virulence factors (Christine et al., 2005). Interestingly, the DsbA2 homologue we first reported for $C$. crescentus (Jameson-Lee et al., 2011) has recently been studied in more detail by the Beckwith group (Cho et al., 2012). They named the protein $\mathrm{ScsC}$ as it was found in an operon containing a novel DsbD that had been named ScsB. Their studies confirmed that $\mathrm{Scs} C$ was a homodimer and exhibited protein disulfide isomerase activity. However, we were surprised that they reported ScsC was an orthologue of DsbC based on phylogenetic criteria (Cho et al., 2012). This contrasts with our conclusion based on phylogenetic criteria, including the conserved 27 amino acid dimerization domain sequence, as well as structural biology (crystal structure) considerations (Jameson-Lee et al., 2011; Kpadeh et al., 2013). First, superimpositions of the crystal structure of a DsbA2 orthologue from Silicibacter (PBD 3gyk) onto DsbA revealed structural identity that was not observed with the DsbC structure. Using Phyre simulations, DsbA2 from L. pneumophila also precicely fit onto the structure of DsbA and not onto DsbC (Jameson-Lee et al., 2011). The similarities between DsbA2 and DsbA and differences with DsbC are obvious from Figure 6. Finally, the NCBI site now shows that DsbA2/Com1 is a distinct clade that evolved from DsbA and not from DsbC. The Beckwith group did however note that like the DsbDs of $L$. pneumophila, the DsbD of C. cresentus was also essential.

DsbA2 is found in bacteria containing type IVb secretion systems including Rickettsia, Legionella, Bartonella, Anaplasma, Brucella, Ehrlichia, Coxiella, and Agrobacterium. Most of these genera have doubling times of $2 \mathrm{~h}$ or more, which raises the possibility that in fast-growing bacteria, the DsbA/DsbC system is required to accelerate correct disulfide bond formation to enable protein folding to keep pace with cell division. This possibility seems to be supported by growth rate differences noted with $E$. coli strains carrying single mutations in $d s b A$ and double 
mutations ( $d s b A d s b C$ and $d s b A d s b D)$ (Vertommen et al., 2008). In this regard, DsbA accelerates disulfide bond formation between consecutive cysteines as most nascent polypeptides enter the periplasm (Zapun et al., 1993; Kadokura \& Beckwith, 2009; Kadokura \& Beckwith, 2010). However, in other proteins, disulfide bonds may form after the protein has entered the periplasm (Kadokura \& Beckwith, 2009) or require correction of mispaired disulfides by DsbC (Denoncin et al., 2010). We suspect that in those slow-growing bacteria expressing DsbA2, such as $L$. pneumophila, disulfide bonds might be introduced after proteins enter the periplasm, which is supported by the efficiency and diversity of proteins captured by the DsbA2 P198T mutant protein (Jameson-Lee et al., 2011). It follows that the apparent essentiality of $d s b A 2$ is due to both its bifunctional nature and an absence of any backup system to repair mis-oxidized proteins that would accumulate to toxic levels in the periplasm. We also noted that overexpression of the DsbA2P198T mutant protein rendered L. pneumophila noninfectious by interfering with assembly and function of the T4SSs (Jameson-Lee et al., 2011). Since $d s b A 2$ was also essential in strains with mutations in dot/icm structural genes, we concluded that DsbA2 must be associated with proper folding of other proteins whose functions might also be essential, including OmpS porin and periplasmic Hsp60 (Butler et al., 1985; Fernandez et al., 1996; Garduno et al., 1998; Jameson-Lee et al., 2011).

The cell envelope of the prokaryotic cell is a major line of defense against environmental challenges (Hatahet et al., 2014). It is also a major site for the maturation of proteins exported from the cytoplasm to the periplasm, outer membrane and to the external environment. In many bacteria, the enzymes that catalyze disulfide bond formation form a distinct pathway from those enzymes that catalyze disulfide bond isomerization. The major argument for support of separate pathways has been that it prevents futile cycling of reducing equivalents from the cytoplasm. 
However, it seems clear from our work that the two processes can be carried out by a single protein, without apparent drain on reducing equivilents. A single system is also supported by examples of the dual activities ascribed to eukaryotic PDI, a four domain oxidoreductase capable of performing thiol oxidation as well as disulfide isomeration (Tian et al., 2006), and rational genetic design experiments in prokaryotes (Segatori et al., 2004). These studies raise the possibility that some bacteria may catalyze oxidation and isomerization by a single protein in the same compartment without any interference, hence supporting the existence of the alternative DsbA2 system discovered in our laboratory.

\subsection{DsbA2 of L. pneumophila differs from canonical DsbA of E. coli}

The results of our studies show that DsbA2 of L. pneumophila, which contains a 56amino-acid N-terminal extension relative to DsbA of E. coli, forms a homodimer in $L$. pneumophila and displays protein disulfide isomerase activity in both in vivo and in vitro assays. Our lab has previously reported that DsbA2 behaved similarly to DsbA by catalyzing the formation of disulfide bonds in secreted proteins of L. pneumophila (Jameson-Lee et al., 2011). Consistent with DsbA-like activity, a DsbA2 cis-proline (P198T) mutant protein formed stable disulfide cross-linked complexes with substrate proteins (Jameson-Lee et al., 2011). Only the disulfide is capable of capturing substrate proteins (Kadokura et al., 2004). In addition, DsbA2 exhibits protein disulfide isomerase activity similar to $\mathrm{Ec} \mathrm{DsbC}$. While the measured reduction potentials of DsbA and DsbC are similar (Hatahet et al., 2014), a stark structural difference between the two is found in domain organization of DsbC. Taken together; it appears that structural and kinetic barriers maintain the segregation of reducing and oxidizing pathways. For instance, the homodimeric arrangement of DsbC exerts steric constraints that preclude its 
interaction with and subsequent oxidation by DsbB (Segatori et al., 2006). Interestingly, DsbA2 is a homodimer, which interacts with either DsbB of L. pneumophila when either Lpn DsbD is present, suggesting its structural and functional distinctiveness from the canonical DsbA.

\subsection{How are the redox forms maintained?}

In support of the above suggestion, we determined that DsbA2 was present as a mixture of reduced thiol and disulfide forms in vivo (Jameson-Lee, UVa thesis 2012, Kpadeh et al., 2013), which contrasts with DsbA and DsbC, which are mostly oxidized and reduced, respectively (Shouldice et al., 2011). We determined by gel filtration that the mixture of oxidized and reduced forms was not due to equilibrium of monomer and dimer forms. Our studies do not rule out the possibility that each arm of the DsbA2 dimer might contribute to the mixture, one as the disulfide and the other as the free thiol. Our studies suggest that structural differences within the thioredoxin folds of DsbA2 might render them more accessible to the DsbB oxidases of $L$. pneumophila. In this regard, L. pneumophila expresses two alleles, DsbB1 and DsbB2 (also known as LidJ) (Conover et al., 2003) that show sequence divergence from E. coli DsbB. As mentioned above, the N-terminal domain of DsbA2 consists of 57 additional amino acids which may contribute to steric hindrance, thus preventing interaction with DsbB in the absence of either Lpn DsbDs. Specifically, deletion of the dimerization domain, which resulted in a monomeric DsbA2 (DsbA2 $\Delta \mathrm{N})$ was structurally different and interacted with Ec DsbB, and both DsbBs of $L$. pneumophila. Due to the distinct biology of Lpn DsbA2 reported thus far, we suggest that Lpn DsbBs can accommodate interaction with DsbA2 at a certain phase of the growth cycle.

As previously reported, L. pneumophila also expresses two alleles of DsbD (Jameson-Lee et al., 2011). We suggest that these competing oxidases and reductases interact with DsbA2 at 
differing efficiencies to maintain equilibrium between oxidized and reduced forms. Interestingly, Segatori et al. (2004) showed that a chimera composed of the dimerization domain of DsbC fused to the catalytic domain of DsbA also existed as a mixture in E. coli and displayed bifunctional activity, including partial complementation of a $d s b A$ mutant for motility (Segatori et al., 2004). Since DsbA2 did not restore motility to a $d s b A$ mutant, DsbA2 is either a poor substrate of DsbB or a good substrate of DsbD. Our studies found that DsbA2 is maintained as the free thiol in $E$. coli by DsbD and efficiently complemented a DsbC mutant in the PDI detector assay. By demonstrating that DsbA2 $\Delta \mathrm{N}$ was able to complement a $d s b A$ mutant of $E$. coli, we conclude that the lack of interaction between DsbA2 and DsbB is most likely the result of steric hindrance by the homodimer as well as efficient interaction with EcDsbD. Interestingly, reconstitution of Lpn $d s b$ genes in E. coli null mutant (RGP438) validated the redox status of DsbA2 as seen in Legionella (mixture of oxidized and reduced forms), thus restoring motility in E. coli. This finding supports our hypothesis that the reduced redox status observed by DsbA2 in E. coli is due to the efficient interaction of EcDsbD with DsbA2; thereby supporting the structurally differences from Lpn DsbDs.

Also, growth assessment of these genetically altered E. coli strains showed attenuation in growth overtime when expressed (Figure 22). Although we did not include data for E. coli strain JP114, we observed no difference in in vitro growth compared to RGP438. This prompted us to use the virulent DH5 $\alpha$ strain as a positive control in the growth assessment assay. It is important to indicate that the growth defect seen by the reconstituted strains may not be solely due to the effect of Lpn DsbDs, but also due to the contributing effect of the necessary antibiotics required for retention of the plasmids or to over expression of recombinant proteins. 
In addition, our findings thus raise a lot of questions about localization and structural differences, as other bacteria containing DsbA orthologues, such as pathogenic bacterium $N$. meningitidis, has three DsbA proteins, which differ in terms of localization, redox properties, and surface characteristics (Tinsley et al., 2004; Lafaye et al., 2008 \& 2009). Particularly, in certain bacteria, such as M. tuberculosis, DsbA is not re-oxidized by DsbB, but by a membrane protein homologous to eukaryotic vitamin K epoxide reductase (VKOR) (Dutton et al., 2008), which support our view that there are many variations and strategies to maintain extracytoplasmic redox associated with management of disulfide bond formation and protein folding.

\subsection{Functional activity of homodimeric DsbA2}

Our studies suggest that DsbA2 is not a homodimeric DsbA as demonstrated with the $E$. coli DsbC-DsbA chimera. We suggest that key amino acid differences in the cis-proline domain (TPA versus VPA in DsbA) similar to DsbC may favor PDI reductase activity. In the insulin reductase assay, DsbA2 and even the DsbA2 $\Delta \mathrm{N}$ monomer exhibited reductase characteristics more similar to those of DsbC than to those of DsbA (Fig. 11). Thus, the DsbA2 lineage appears to be a unique adaptation that combines both oxidase and reductase functions into a single protein in situ. To explore the basis for this adaptation, we have replaced the E. coli $d s b B$ and $d s b D$ genes with alleles from L. pneumophila, and we observed that DsbA2 did not interact with either Lpn DsbB1 or Lpn DsbB2, but did interact with both Lpn DsbD1 and Lpn DsbD2 to restore PDI activity. These results suggested that the combined activity of both Lpn DsbB and Lpn DsbD is required for DsbA2 to exist in the mixture of oxidized and reduced forms.

A number of refolding assays have been developed to demonstrate in vitro PDI activity (Berkmen et al., 2005; Kadokura \& Beckwith, 2009; Kadokura \& Beckwith, 2010). These assays 
often rely on refolding of scrambled enzymes like RNase I that contains consecutive as well as nonconsecutive disulfide bonds to restore RNase activity (Messens et al., 2007; Kadokura \& Beckwith, 2010). We can speculate that most DSB enzymes are technically bifunctional (oxidase and reductase). For example, EcDsbA is mostly oxidized but contains some reduced forms that do not exhibit PDI ativity. This may suggest that the bifunctional nature of a DSB enzyme depends on the oxidant (s) and reductant (s), respectively. In addition, several mutational analyses and structural studies (Bader et al., 2001; Arredondo et al., 2009) have shown that formal oxidases can be made into isomerases and vice versa, through small point mutations, over-production of an enzyme, or by subtly changing the redox environment. These experiments suggest that while a strict division of oxidase and reductase function is necessary to prevent a futile cycle, in reality there exists a gradient between oxidase and reductases and that enzymes can be coaxed toward one end or the other.

On the other hand, Lpn DsbA2, which is proposed in this study as a naturally bifunctional enzyme, is unique in that while a P198T mutation trapped interacting substrates similar to the $E$. coli DsbA monomer, it is equally reduced and oxidized in vivo and phylogentically distinct from DsbA and DsbC. This could suggest that DsbA2 may be a natural scaffold that has been adapted through evoulution to contain both oxidase and PDI activity.

PDI assays can yield mixed results, such as DsbA refolding RNase I (Messens et al., 2007). One advantage to the use of the PDI detector MBla for these assays is that the protein does not require denaturation prior to assay and the consecutive disulfide bonds produced are the result of DsbA activity (Ren \& Bardwell, 2011). In this folding assay, gain of enzyme activity is readily detected with the chromogenic penicillin substrate nitrocefin. DsbA2, but not DsbA1 efficiently restored the correct nonconsecutive disulfide bond pairing required for protein folding and Bla 
activity. The adaptation of this assay to a microplate format should enable development of rapid screens of proteins for PDI activity. In our hands the deep red color of the cleaved $\beta$-lactam is easily distinguished by visual observation. We have shown that the DsbA2 protein of $L$. pneumophila is a homodimer and is capable of delivering disulfide bonds to native unfolded proteins or through PDI activity to repair inappropriate disulfide bonds. While the DsbA2 lineage represents a subset of the DsbA family, the dimerization domain and threonine substitution for valine in the cis-proline domain are more similar to DsbC and represent unique adaptations that may facilitate or enhance the bifunctional activity of the enzyme.

\subsection{Reconstitution of $L$. pneumophila dsb genes in E. coli background}

We described above that expression of DsbA2 complemented a $\Delta d s b C$ mutant in a PDI/ reductase detector assay in E. coli (Ren and Bardwell, 2011, Kpadeh et al., 2013). On the other hand, $\operatorname{DsbA} 2 \Delta \mathrm{N}$ complemented a $d s b A$ mutant in soft agar motility assay in an $E$. coli background (Kpadeh et al., 2013). Knowing these two distinct pathways (oxidative and reductive) are independent in E. coli, and any cross talk may result in a futile cycle, we suggested that the DsbA2 paradigm in L. pneumophila is completely novel. Most importantly, none of the $d s b$ genes studied to date in other bacteria have been found to be essential (Heras et al., 2009); however, all of the $d s b$ genes in L. pneumophila seem to be essential except for $d s b A 1$. In order to address whether there is any difference in the efficiency of protein interactions between the oxidized DsbA2 and L. pneumophila oxidases (DsbB1 and DsbB2) in contributing to the maintenance of disulfide bond folding in the periplasm, we used a surrogate system to address the roles of two DsbB alleles (DsbB1 and DsbB2) in the maintenance of DsbA2 oxidase activity. An interaction assay of L. pneumophila $d s b$ genes; $d s b A 2 \Delta \mathrm{N}, d s b A 1$ or $d s b A 2$ with either $d s b B 1$ or $d s b B 2$ in an 
E. coli $d s b$ double mutant $(\Delta d s b A d s b B)$ obtained from James Bardwell showed DsbA1 and DsbA2 $\Delta \mathrm{N}$, but not DsbA2 interact with both L. pneumophila DsbBs to complement motility in the E. coli double mutant (Figure 17). Findings from these experiments validated our previous $d s b A$ complementation result with DsbA2 $\Delta \mathrm{N}$ (Kpadeh et al., 2013), suggesting that DsbA2 $\Delta \mathrm{N}$ partners with DsbB1 and DsbB2 for its oxidative activity; DsbB2 (LidJ) seens to be slightly more efficient. Since DsbA2 $\Delta \mathrm{N}$ does not exist in nature and DsbA2 is oxidized in vivo in $L$. pneumophila; we speculated that DsbA2 must interact with L. pneumophila DsbBs. However, in the E. coli background, EcDsbD efficiently maintained DsbA2 as the free thiol, thus preventing oxidation by DsbBs. We found that by expressing the Lpn DsbDs in E. coli that their poor efficiency in reducing DsbA2 permitted interaction and oxidation by Lpn DsbBs in this background. Future studies might examine the structural interactions between DsbBs and DsbA2 to confirm these interactions.

Although we did not investigate all of the possibilities stated above, knowing that DsbA2 contains a N-terminal extension, which is important for DsbA2 to form a homodimer in Lpn that behaves similarly to DsbC (dimer) by displaying protein disulfide isomerase activity in both in vivo and in vitro assays (Kpadeh et al., 2013). We sought to reveal how DsbA2 interactions with the reductants (DsbDs) could contribute to or moderate its interaction with DsbBs in vivo. Like DsbC, DsbA2 is a disulfide isomerase that is thought to correct wrongly formed disulfide bonds to produce a functional protein. DsbC is part of the reductive pathway that is linked, via DsbD, to the thioredoxin system of the cytosol (Rietsch et al., 1997). DsbC is noted to have an active-site cysteine pair with a redox potential almost as low as that of DsbA (Zapun et al., 1993; Wunderlich et al., 1993; Zapun et al., 1995), and should at least be sufficient to catalyze both disulfide-bond formation and rearrangement in the cell without the need for a specialized oxidant. 
However, this was not the case with DsbC as described by (Segatori et al., 2004); even though it contains high oxidation activity per se, in the periplasmic space it is maintained exclusively in the reduced state, and therefore can only catalyze disulfide-bond rearrangement. In contrast, we have demonstrated in the lab that periplasmic DsbA2 exists as a mixture of oxidized and reduced redox forms in L. pneumophila (Kpadeh et al., 2013), suggesting DsbA2 is able to associate with both Lpn DsbBs and Lpn DsbDs. We think this interaction is solely due to its moderate interaction with Lpn DsbDs, which are divergent to E. coli DsbD, which fully reduced DsbA2 when E. coli DsbB is present and not Lpn DsbBs.

DsbD consists of three domains: a N-terminal periplasmic domain (DsbD $\alpha$ ), a C-terminal periplasmic domain (DsbD $\gamma$ ), and an intervening hydrophobic membrane-embedded domain $(\mathrm{DsbD} \beta)$ (see Figs. $35 \& 36)$. Each of the three domains contains two redox-active cysteines, essential for the protein's function (Stewart et al., 1999). DsbD homologues are found in many other bacteria (Katzen et al., 2002). In E. coli, enzymes including DsbC, a protein that is involved in disulfide bond isomerization, DsbG, which restores the reduced form of cysteines that have been oxidized to sulfenic acid (Depuydt et al., 2009), and CcmG, which reduces the oxidized cysteines of apocytochrome $c$ so as to allow heme binding (Metheringham et al., 1995; Fabianek et al., 1998) derive their reducing power from cytoplasmic thioredoxin via the cytoplasmic membrane protein DsbD (Fig. 36). However, many bacteria including L. pneumophila, express protein homologues that comprise the cytoplasmic, intervening hydrophobic membraneembedded and a N-terminal periplasmic domains, or only the cytoplasmic domain of DsbD as reported for CcdA of Rhodobacter capsulatus which reduces proteins that are homologues of CcmG (Deshmukh et al., 2000; Katzen et al., 2002). It seems like electrons transfer from DsbD $\alpha$ is enough to reduce its substrates (Gupta et al., 1997; Katzen \& Beckwith, 2000; Collet et al. 
2002; Haebel et al., 2002; Stirnimann et al, 2005). Bioinformatic analyses of Lpn DsbDs, in this study reveal that Lpn DsbD1 N-terminal periplasmic domain is more similar to the E. coli DsbD (Fig. 35) than to the Salmonella Typhimurium encoded homologue of DsbD, scsB (Gupta et al., 1997; Cho et al., 2012), a newly identified distinct class which comprises DsbD homologues with quite different $\alpha$ domains. However, Lpn DsbD2 lacks the N-termial N $\alpha$ domain (Fig. 35, bottom right) found in DsbD1 that in E. coli delivers the reducing equivalents to DsbC. DsbD2 does share domain organization with the $\beta$ and $\gamma$ domains as EcDsbD. A further analysis of Lpn DsbD1 reveals the protein to contain several additional cysteine residues, which compared to EcDsbD could conceivably alter disulfide bonding or other structural changes that would likely influence catalytic efficiency with DsbA2. In summary, these results indicate DsbDs of L. pneumophila are divergent from EcDsbD structurally and functional, and DsbA2 has to moderately interact with both Lpn DsbBs and Lpn DsbDs in order to exhibit a bifunctional activity in vivo. 


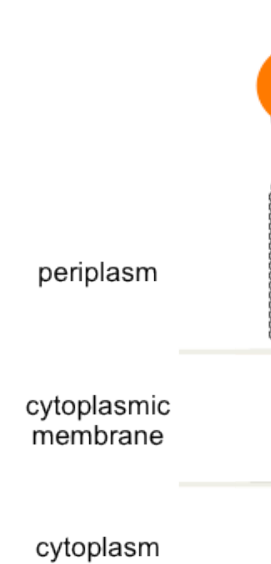

cytoplasm

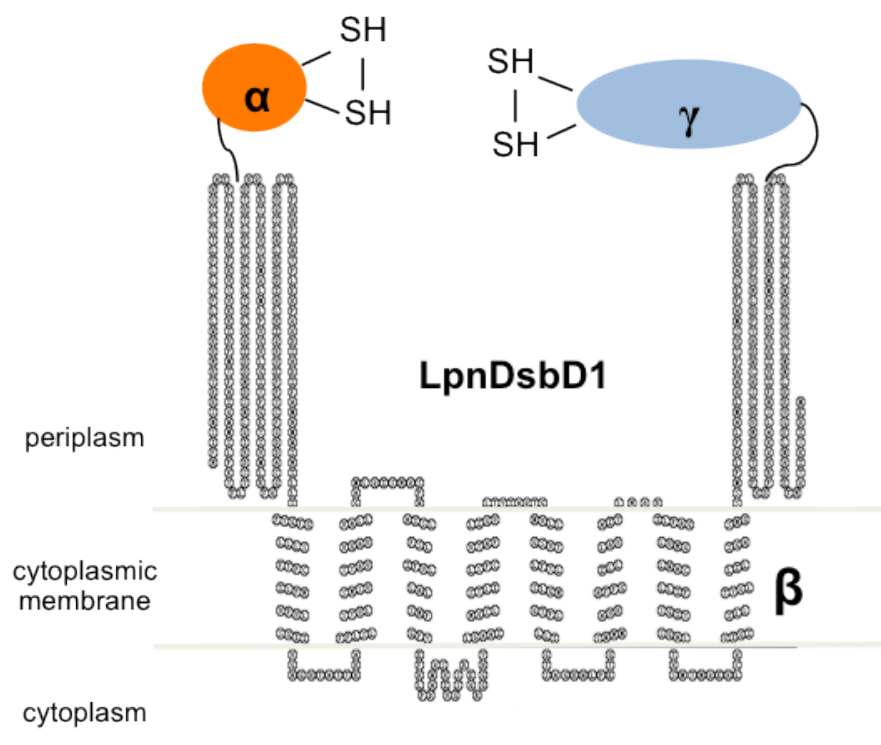

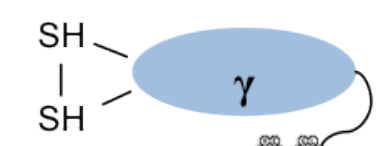

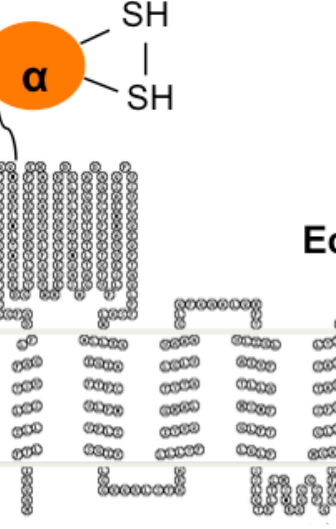

EcDsbD

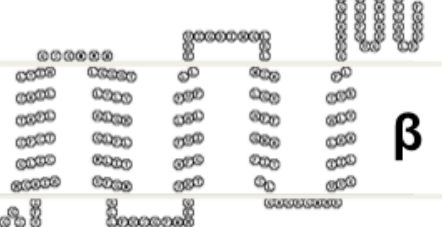

Figure 35. DsbD topological models of E. coli DsbD and Lpn DsbDs. Comparisons between Ec DsbD and Lpn DsbDs depicting the $\alpha, \beta$, and $\gamma$ domains. Lpn DsbD2 lacks the Na domain as compared to EcDsbD and Lpn DsbD1. 


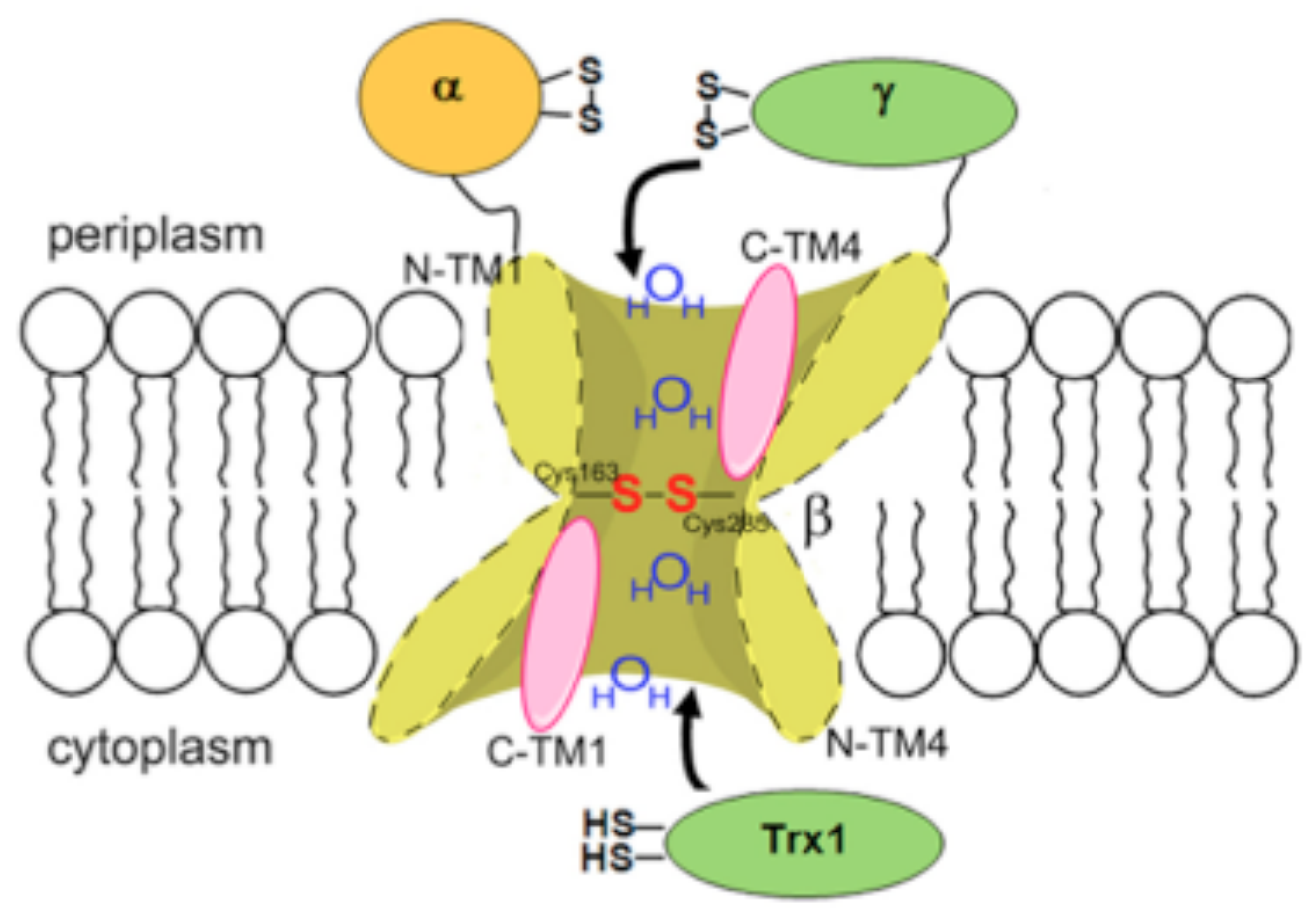

Figure 36. Structural model of E. coli DsbD. The model above shows that $D s b D \beta$ adopts an hourglass-like structure where Cys 163 and Cys285 are in the middle of the two water-exposed cavities. Halves of C-terminal TM1 and TM4 (C-TM1 and C-TM4) are water exposed (pink), while those of $\mathrm{N}$-terminal ones (N-TM1 and N-TM4) are not. Two thioredoxin modules (thioredoxin-1 and DsbD $\gamma$ ) may interact with Cys163 and Cys285 through these two waterexposed cavities. S, sulfur of thiol in a cysteine residue. It is noteworthy that Lpn DsbD2 is missing the $\alpha$ redox arm believed to directly reduce DsbA2. Adapted from Cho et al., 2012. 


\subsection{In vivo characterization of DsbA2 forms for virulence in $L$. pneumophila}

In addition, we characterized the monomer form of oxidoreductase DsbA2, DsbA2 $\Delta \mathrm{N}$, and its contributing role to the bifunctional activity of DsbA2 for virulence in the periplasm of $L$. pneumophila. Consistent with DsbA-like activity, a DsbA2 cis-proline (P198T) mutant protein formed stable disulfide cross-linked complexes with substrate proteins, but not with its dedicated oxidant DsbB (Kadokura et al., 2004; Jameson-Lee et al, 2011), and further exhibited a dominant negative effect that was due to interference with either assembly or function of the Dot/Icm T4SS apparatus (Jameson-Lee et al., 2011). DsbA2 was reported in (Jameson-Lee et al., 2011) to capture some important substrates including, Dot/Icm T4SS core structural proteins DotC, DotG, DotK and IcmX, the periplasmic and surface-localized invasin HtpB (Hsp60), and its cochaperone HtpA (Hsp10), and subunits of Omps of the MOMP complex. It is also important to note that other Dot/Icm proteins that do not contain cysteine residues such as DotD, DotF, IcmW and IcmY, or cytoplasmic proteins that do contain cysteine residues were not captured by DsbA2 P198T mutant protein. The Dot/Icm proteins captured through interaction with P198T muntant were considered essential for assembly and function of the T4SS. Also, L. pneumophila DsbA1 has been reported to be dispensable for growth, motility, and infectivity in cell-based assays and this gene is naturally absent from the genome of $C$. burnetti, which also express $d s b B$ and $d s b D$ genes, while DsbA2 is essential for virulence (Jameson-Lee et al., 2011; Kpadeh et al., 2013).

In order to characterize the importance of the monomer form of DsbA2 on pathogenesis, we utilized DsbA2 $\Delta \mathrm{N}$ as a tool to assess this effect by constructing a P198T mutation in DsbA2 $\Delta \mathrm{N}$ from (Kpadeh et al., 2013) to overcome the apparent essentiality of DsbA2. We examined DsbA2 $\Delta \mathrm{N}$ P198T mutant in vitro and by protein expression on SDS-PAGE and immunoblotting with anti-DsbA2 serum, and no defect in growth was seen. However, we show 
here that expression of the DsbA2 $\Delta \mathrm{N}$ P198T mutant protein in L. pneumophila strain AA100 produced a dominant-negative effect on DsbA2 function resulting to several virulence phenotypes including: (i) defect of intracellular multiplication in amoeba, (ii) a significant defect in attaching and invading HeLa cells similarly to DsbA2 P198T mutant in (Jameson-Lee et al., 2011), and (iii) a greater defect in contact dependent hemolysis of human erythrocytes, all of which suggest the mutant has an incomplete and non-functional Dot/Icm T4SS.

Although amoeba has been one of the most recognized model hosts for evaluating $L$. pneumophila infection and growth intracellularly, our amoeba results led us to further question whether the duration of the bacteria in contact with amoeba has any effect on the results generated. To eliminate any factors that may skew the analysis of the infectivity results, we infected non-phagocytic HeLa cells with our mutant to investigate the virulence of oxidized DsbA2 in vivo. HeLa cell is an established model system to study the invasiveness and biology of L. pneumophila (Garduno et al., 1998a \& Garduno et al., 1998c). We utilized this model to examine adherence and invasion; virulent strains of Legionella are adherent and invasive while nonvirulent strains are adherent but poorly invasive. Invasion is reported to be rapid and does not require de novo bacterial protein synthesis (Garduno et al., 1998a), suggesting that the factors necessary for invasion, which require a properly assembled Dot/Icm T4SS apparatus for their translocation, have to be constitutively expressed by virulent strains. Importantly, in addition to the capture of Dot/Icm components by Jameson-Lee et al. (2011), a wide range or substrate proteins were captured, including enhanced entry proteins (Ehn) that are required for entry into amoeba (Liu et al., 2008), and HtpB (Hsp60), which facilitates attachment and invasion of nonphagocytic cells (Garduno et al., 1998a\&b). Perhaps the reduced attachment and invasion 
observed with monomeric DsbA2 P198T mutant protein might be due to impairment of Ehn and/or HtpB function (s), respectively.

Due to similar activity assessed in DsbA2 P198T mutant, these results suggest that DsbA2 $\Delta \mathrm{N}$ P198T interfered with the function of DsbA2, and resulted in a disruption in the Dot/Icm T4SS complex. In addition, these results indicate that Lpn is dependent on the oxidized form of DsbA2 for the proper folding of periplasmic proteins, including those destined for the assembly into macromolecular structures.

\subsection{Why is DsbA1 maintained in L. pneumophila?}

In many pathogenic bacteria, DsbA-like proteins are important for assembly of virulence factors and for secretion systems that are dependent on disulfide bond formation (Heras et al., 2009). However, previous reports had shown no phenotype for Lpn $d s b A 1$ mutant (Jameson-Lee et al., 2011). This result was not concerning at first since $d s b A 1$ is absent in Coxiella, the most closely related bacteria to Legionella. Since DsbA1 showed enzyme activity in the insulin precipitation assay, and was more oxidizing than reducing similar to EcDsbA, we reexamined the biology of the Lpn $d s b A 1$ mutant. To confirm the role of DsbA1 in the DSB system of $L$. pneumophila, we closely evaluated any difference in in vitro broth culture AA100 WT and $\triangle d s b A 1$ constructs. Interestingly, we detected a defect in pigment production in strains lacking $d s b A 1$. We also examined the ability for $\triangle d s b A 1$ mutant to grow in regular or cys and iron limited BYE media, and no growth difference was detected compared to AA100 WT, suggesting that the defect in pigment production is not contributed by cys or iron acquisition. Pigment production such as melanin is important in microbial pathogenesis because it has been associated with virulence in many microorganisms, and is linked to protection against environmental insults 
(Nosanchuk and Casadevall, 2003). Therefore, the lack of dark-brownish pigment observed following overnight growth in broth might suggest that DsbA1 interacts with protein(s) that are important for modulating the virulence of Lpn. Because DsbA1 appears to have a phenotypic difference from wild type, we wanted to more definitively elucidate the functional activity of DsbA1 for virulence in an infectivity assay. Using L929 cells, we performed plaque assay using different constructs of Legionella AA100 strains including $d s b A 1$ knockout. In these studies, the $\triangle d s b A 1$ mutant demonstrated a hyper-virulent phenotype that disappeared upon complementation with WT DsbA1, indicating a modulating role for Legionella virulence. In further studies utilizing contact-dependent lysis of human erythrocytes, we were able to validate this hypervirulent phenotype demonstrated in L929 cells. All together, the defect in pigment production and the hypervirulent phenotype illustrated in vivo define a role for DsbA1 in Lpn. Noteworthy, this is the first report indicating an important role of DsbA1 in L. pneumophila.

Furthermore, protein capture assays indicated that DsbA1 interacts with fewer substrates in the periplasm of L. pneumophila compared to expression in E. coli. Knowing that capture studies has been very important for the characterization of oxidase function of DsbA in other pathogens, and DsbA2 in L. pneumophila (Jameson-Lee et al, 2011), we can suggest that DsbA1 is not evolutionarily selected against because it contributes to disulfide bond formation in the periplasm. Lastly, we successfully crystalized DsbA1, and comparison analysis of Lpn DsbA1, EcDsbA and EcDsbC showed that Lpn DsbA1 is more similar to EcDsbA structurally than EcDsbC. We concluded from these studies that although DsbA1 may not be as directly involved in protein folding associated with virulence traits. Our studies support a role, perhaps through interference with DsbA2 function, as a modulator of virulence in L. pneumophila. 


\section{Chapter 5. Conclusion}

Our studies contribute new functional knowledge on the biology of the DsbA2 system, a novel class of DSB oxidoreductase common to nearly all bacteria expressing T4SSs and many that do not. We have identified fundamental differences between the L. pneumophila DsbA2 system and the DsbA/DsbC system of E. coli (Fig. 21). These studies have also revealed that both redox forms of DsbA2 are most important and essential - the oxidase or the PDI reductase activity for Dot/Icm T4SS proper assembly and function. In addition, we have reported here that there is considerable diversity in DSB proteins and their biology, which our work has stimulated the possibility to explore these systems in other bacteria contributing to macromolecular assembly and function of T4SSs. The redundancy of $d s b$ genes $(d s b B 1, d s b B 2, d s b D 1$ and $d s b D 2)$ in $L$. pneumophila may provide a cumulative advantage, each contributing to the global quantity of a protein involved in a specific cellular process (Thomas, 1993). It is highly likely that under certain growth conditions, the presence of a single $d s b B$ gene or $d s b D$ gene is not sufficient to drive disulfide bond formation and that efficient oxidative folding requires the presence of both $d s b B$ s or $d s b D$ s. Alternatively, possessing two $d s b B$ or $d s b D$ genes may allow a broader spectrum of regulation than a single one, the expression of two oxidant/redundant operons may be differently modulated according to the environmental conditions, which would de facto give rise to more individualized functions for these two genes.

We also find it interesting that DsbA2 related proteins are present in a number of bacterial species instead of the DsbA/DsbC system as a means to manage extracytoplasmic disulfide bonding and protein folding (e.g. Coxiella burnetti which only contains DsbA2 and lacks both DsbA1 and DsbC). This genetically diverse group also includes most intracellular parasites that express T4SS, all of which grow very slowly compared to E. coli. Perhaps DsbA2 represents an 
evolutionary adaptation that is more efficient in orchestrating disulfide bond formation and protein folding that suits the lifestyles of slow-growing bacteria. Finally, we speculate $d s b A 2$ mutants can be used to probe how Dot/Icm system becomes activated following contact with a host cell, thus making a significant impact in the field of microbiology and microbial pathogenesis. Thus, targeting of disulfide bond formation pathways appears to be an attractive approach for the development of antimicrobials and anti-virulence agents. The future may yield inhibitors of these pathways of both basic and applied interest as some reports in literature have begun to address this concept (Shouldice et al, 2010b; Fruh et al., 2010). With these ideas in mind, further investigations will surely reveal the roles of DsbA2 for the proper folding of targeted-captured substrates, and essentiality during environmental survival and pathogenesis in humans. 


\section{References}

Abu Kwaik, Y. and Pederson, L. L. (1996). The use of differential display-PCR to isolate and characterize a Legionella pneumophila locus induced during the intracellular infection of macrophages. Mol Microbiol 21, 543-556.

Abu Kwaik, Y., Gao, L. Y., Stone, B. J., Venkataraman, C. \& Harb, O. S. (1998). Invasion of protozoa by Legionella pneumophila and its role in bacterial ecology and pathogenesis. Appl Environ Microbiol 64, 3127-3133.

Alli, O. A., Gao, L. Y., Pedersen, L. L., Zink, S., Radulic, M., Doric, M. \& Abu Kwaik, Y. (2000). Temporal pore formation-mediated egress from macrophages and alveolar epithelial cells by Legionella pneumophila. Infect Immun 68, 6431-6440.

Anfinsen, C. B. and Haber, E. (1961). Studies on the reduction and re-formation of protein disulfide bonds. $J$ Biol Chem 236, 1361-1363.

Anfinsen, C. B., Haber, E., Sela, M., \& White, Jr., F. H. (1961). The kinetics of formation of native ribonuclease during oxidation of the reduced polypeptide chain. Proc. Natl. Acad. Sci. USA 47, 1309-1314.

Anfinsen, C. B. (1973). Principles that govern the folding of protein chains. Science 181, 223230.

Arredondo, S. A., Chen, T. F., Riggs, A. F., Gilbert, H. F., \& Georgiou, G. (2009). Role of dimerization in the catalytic properties of the Escherichia coli disulfide isomerase DsbC. J Biol Chem 284, 23972-23979.

Arts, I. S., Ball, G., Leverrier, P., Garvis, S., Nicolaes, V., Vertommen, D., Ize, B., Dufe, V. T., Messens, J., Voulhoux, R., Collet, J. F. (2013). Dissecting the machinery that introduces disulfide bonds in Pseudomanas aeruginosa. mBio 4, 6:e00912-13. 
Backert, S. and Meyer, T. F. (2006). Type IV secretion systems and their effectors in bacterial pathogenesis. Curr Opin Microbiol 9, 207-217.

Bader, M. W., Hiniker, A., Regeimbal, J., Goldstone, D., Haebel, P. W., Riemer, J., Metcalf, P. \& Bardwell, J. C. A. (2001). Turning a disulfide isomerase into an oxidase: DsbC mutants that imitate DsbA. EMBO J 20, 1555-1562.

Baek, M. C., Kim, S. K, Kim, D. H., Kim, B. K. \& Choi, E. C. (1996). Cloning and sequencuig of the Klebsiella K-36 astA gene, encoding an arylsulfate sulfotransferase. Microb Immun 40, 531-537.

Banga, S., Gao, P., Shen, X., Fiscus, V., Zong, W. X., Chen, L. \& Luo, Z. Q. (2007). Legionella pneumophila inhibits macrophage apoptosis by targeting pro-death members of the Bc12 protein family. Proc Natl Acad Sci USA 104, 5121-5126.

Bardill, J. P., Miller, J. L. \& Vogel, J. P. (2005). IcmS-dependent translocation of SdeA into macrophages by the Legionella pneumophila type IV secretion system. Mol Microbiol 56, 90-103.

Bardwell, J. C., McGovern, K. \& Beckwith, J. (1991). Identification of a protein required for disulfide bond formation in vivo. Cell 67, 581-589.

Bardwell, J. C. A., Lee, J. O., Jander, G., Martin, N., Belin, D. Band, Beckwith, J. (1993). A pathway for disulfide bond formation in vivo. Proc Natl Acad Sci USA 90, 1038-1042.

Bardwell, J. C. A. and Beckwith, J. (1993). The bonds that tie: catalyzed disulfide bond formation. Cell 74:769-771.

Beckwith, J. (2007). What lies beyond uranus? Preconceptions, ignorance, serendipity and suppressors in the search for biology's secrets. Genetics 176, 733-740. 
Benson, R. F. and Fields, B. S. (1998). Classification of the genus Legionella. Semin Respir Infect 13, 90-99.

Berger, K. H., and R. R. Isberg. (1993). Two distinct defects in intracellular growth complemented by a single genetic locus in Legionella pneumophila. Mol. Microbiol 7, 7-19.

Berk, S. G., R. S. Ting, G. W. Turner, \& R. J. Ashburn. (1998). Production of respirable vesicles containing live Legionella pneumophila cells by two Acanthaamoeba Spp. Appl Environ Microbiol 64, 279-286.

Berkmen, M., Boyd, D., \& Beckwith, J. (2005). The nonconsecutive disulfide bond of Escherichia coli phytase (AppA) renders it dependent on the protein-disulfide isomerase, DsbC. $J$ Biol Chem 280, 11387-11394.

Bessette, P. H., Cotto, J. J., Gilbert, H. F., \& Georgiou, G. (1999). In vivo and in vitro function of the Escherichia coli periplasmic cysteine oxidoreductase DsbG. J Biol Chem. 274, 7784 -7792.

Bitar, D. M., M. Molmeret \& Y. Abu Kwaik. (2004). Molecular and cell biology of Legionella pneumophila. Int J Med Microbiol 293, 519-527.

Blackman, J. A., M. D. Hicklin, \& F. W. Chandler. (1978). Special expert pathology panel. Legionnaires' disease: pathological and historical aspects of a "new" disease. Arch. Path. Lab. Med. 102:337-343.

Bornstein, N., D. Marmet, M. H. Dumaine, M. Surgot, \& J. Fleurette. (1991). Detection of flagella in 278 Legionella strains by latex reagent sentisized with antiflagellum immunoglobulins. J Clin Microbiol 29, 953-960.

Brand, B. C., Sadosky, A. B., \& Shuman, H. A. (1994). The Legionella pneumophila icm locus: a set of genes required for intracellular multiplication in human macrophages. Mol Microbiol. 14, 797- 808 . 
Brenner, D. J., J. C. Feeley, \& R. E. Weaver. (1979). Legionellaceae . p. 279-288. In N. R. Kreieg, J. G. Holt (ed), Bergey's manual of systematic bacteriology. Volume 1. 1984. Williams \& Wilkins, Baltimore, MD.

Brenner, D. J. (1986). Classification of Legionellaceae. Current status and remaining questions. Isr J Med Sci 22, 620-632.

Brieland, J., M. McClain, L. Heath, C. Chrisp, G. Huffnagle, M. LeGendre, M. Hurley, J. Fantone, \& Engleberg, C. (1996). Coinoculation with Hartmannella model of Legionnaires' disease. Infect Immun 64, 2449-2456.

Bruggemann, H., A. Hagman, M. Jules, O. Sismeiro, M. A. Dillies, C. Gouyette, F. Kunst, M. Steinert, K. Heuner, J. Y. Coppee, \& Buchrieser, C. (2006). Virulence strategies for infecting phagocytes deduced from the in vivo transcriptional program of Legionella pneumophila. Cell Microbiol 8, 1228-1240.

Butler, C. A., Street, E. D., Hatch, T. P., \& Hoffman, P. S. (1985). Disulfide bonded outer membrane proteins in the genus Legionella. Infect Immun 48, $14-18$.

Byrne, B. and M. S. Swanson. (1998). Expression of Legionella pneumophila virulence traits in response to growth conditions. Infect Immun 66, 3029-3034.

Canfield, R. E. and Anfinsen, C. B. (1963). Chromatography of pepsin and chymotrypsin digests of egg white lysozyme on phosphocellulose. J Biol Chem 238, 2684-2690.

Carvalho, A. P., Fernandes, P. A. \& Ramos, M. J. (2006). Similarities and differences in the thioredoxin superfamily. Prog Biophys Mol Biol 91, 229-248. 
Catrenich, C. E. and W. Johnson. (1989). Characterization of the selective inhibition of growth of virulent Legionella pneumophila by supplemented Mueller-Hinton medium. Infect Immun 57, 1862-1864.

Chacinska, A., Koehler, C. M., Milenkovic, D., Lithgow, T. \& Pfanner, N. (2009). Importing mitochondrial proteins: machineries and mechanisms. Cell 138, 628-644.

Chandler, F. W., B. M. Thomason, \& G. A. Hebert. (1980). Flagella on Legionnaires' disease bacteria in the human lung. Ann Intern Med 93, 715-716.

Charpentier, X., Gabay, J. E., Reyes, M., Zhu, J. W., Weiss, A., \& Shuman, H. A. (2009). Chemical genetics reveals bacterial and host cell functions critical for type IV effector translocation by Legionella pneumophila. PLoS Pathog 5, e1000501.

Chen, J., K. S. de Felipe, M. Clarke, H. Lu, O. R. Anderson, G. Segal, \& H. A. Shuman. (2004). Legionella effectors that promote nonlytic release from protozoa. Science 303, 13581361.

Chien, M., Morozova, I., Shi, S., Sheng, H., Chen, J., Gomez, S. M., Asamani, G., Hill, K., Nuara, J., \& other authors. (2004). The genomic sequence of the accidental pathogen Legionella pneumophila. Science 305, 1966-1968.

Christie, P. J., Atmakuri, K., Krishnamoorthy, V., Jakubowski, S., \& Cascales, E. (2005). Biogenesis, architecture, and function of bacterial type IV secretion systems. Annu Rev Microbiol $59,451-485$.

Cho, S. H., Parsonage, D., Thurston, C., Dutton, R. J., Poole, L. B., Collet, J. F., \& Beckwith, J. (2012). A new family of membrane electron transporters and its substrates, including a new envelope peroxiredoxin, reveal a broadened reductive capacity of the oxidative bacterial cell envelope, MBio 3 (2012), http://dx.doi.org/10.1128/mBio.00291-11. 
Cirillo, J. D., S. Falkow, \& Tompkins, L. S. (1994). Growth of Legionella pneumophila in Acantamoeba castellanii enhances invasion. Infect Immun 62, 3254-3261.

Coers, J., C. Monaham, \& C. R. Roy. (1999). Modulation of phagosome biogenesis by Legionella pneumophila creates an organelle permissive for intracellular growth. Nat Cell Biol 1, 451-453.

Collet, J. F., Riemer, J., Bader, M. W., \& Bardwell, J. C. (2002). Reconstitution of a disulfide isomerization system. J Biol Chem 277, 26886-26892.

Conover, G. M., Derré, I., Vogel, J. P. \& Isberg, R. R. (2003). The Legionella pneumophila LidA protein: a translocated substrate of the Dot/Icm system associated with maintenance of bacterial integrity. Mol Microbiol 48, 305-321.

Dailey, F. E. and Berg, H. C. (1993). Mutants in disulfide bond formation that disrupt flagellar assembly in Escherichia coli. Proc Natl Acad Sci USA 90, 1043-1047.

Darby, N. J. and Creighton, T. E. (1995). Catalytic mechanism of DsbA and its comparison with that of protein disulfide isomerase. Biochemistry 34, 3576-3587.

de Felipe, K. S., Glover, R. T., Charpentier, X., Anderson, O. R., Reyes, M., Pericone, C. D. \& Shuman, H. A. (2008). Legionella eukaryotic-like type IV substrates interfere with organelle trafficking. PLoS Pathog 4, e1000117.

Denoncin, K., Vertommen, D., Paek, E., \& Collet, J. F. (2010). The protein disulfide isomerase DsbC cooperates with SurA and DsbA in the assembly of the essential $\beta$-barrel protein LptD. $J$ Biol Chem 285, 29425-29433.

Depuydt, M., Leonard, S. E., Vertommen, D., Denoncin1, K., Morsomme, P., Wahni, K., Messens, J., Carroll, K. S., Collet, J. F. (2009). A periplasmic reducing system protects single cysteine residues from oxidation. Science 326, $1109-1111$. 
Deshmukh, M., Brasseur, G., \& Daldal, F. (2000). Novel Rhodobacter capsulatus genes required for the biogenesis of various c-type cytochromes. Mol Microbiol 35, 123-138.

Doleans, A., Aurell, H., Reyrolle, M., Lina, G., Freney, J., Vandenesch, F., Etienne, J. \& Jarraud, S. (2004). Clinical and environmental distributions of Legionella strains in France are different. J Clin Microbiol 42, 458-460.

Dutton, R. J., Boyd, D., Berkmen, M., \& Beckwith, J. (2008). Bacterial species exhibit diversity in their mechanisms and capacity for protein disulfide bond formation. Proc Natl Acad Sci USA 105, 11933-11938.

Dutton, R. J., Wayman, A., Wei, J. R., Rubin, E. J., Beckwith, J. \& Boyd, D. (2010). Inhibition of bacterial disulfide bond formation by the anticoagulant warfarin. Proc Natl Acad Sci USA 107, 297-301.

Edelstein, P. H. (1981). Improved semiselective medium for isolation of Legionella pneumophila from contaiminated clinical and environmental specimens. J Clin Microbiol 14, 298-303.

Ewann, F. and Hoffman, P. S. (2006). Cysteine metabolism in Legionella pneumophila: characterization of an L-cystine-utilizing mutant. Appl Environ Microbiol 72, 3993-4000.

Fabianek, R. A., Hennecke, H., \& Thöny-Meyer, L. (1998). The active-site cysteines of the periplasmic thioredoxin-like protein CcmG of Escherichia coli are important but not essential for cytochrome $c$ maturation in vivo. J Bacteriol 180, 1947-1950.

Faulkner, G. and Garduno, R. A. (2002). Ultrastructural analysis of differentiation in Legionella pneumophila. J Bacteriol 184, 7025-7041.

Feeley, J. C., Gibson, R. J., Gorman, G. W., Langford, N. C., Rasheed, J. K., Mackel, D. C. \& Baine, W. B. (1979). Charcoal-yeast extract agar: primary isolation medium for Legionella pneumophila. J Clin Microbiol 10, 437-441. 
Fernandez, R., Logan, S., Lee, S., \& Hoffman, P. S. (1996). Elevated levels of Legionella pneumophila stress protein Hsp60 early in infection of human monocytes and L929 cells correlate with virulence. Infect Immun 64, 1968 -1976.

Fields, B. S. (1996). Molecular ecology of legionellae. Trends in Microbiol 4, 286-290.

Fields, B. S., Benson, R. F. \& Besser, R. E. (2002). Legionella and Legionnaires' disease: 25 years of investigation. Clin Microbiol Rev 15, 506-526.

Findlay, B. B., and S. Falkow. (1997) Common themes in microbial pathogenesis revisted. Microbiol Mol Biol Rev 61, 136-169.

Fliermans, C. W., W. B. Cherry, L. H. Orrison, S. J. Smith, D. L. Tison, \& Pope, D. H. (1981). Ecological distribution of Legionella pneumophila. Appl Environ Microbiol 41, 9-16.

Franco, I. S., Shuman, H. A., \& Charpentier, X. (2009). The perplexing functions and surprising origins of Legionella pneumophila type IV secretion effectors. Cell Microbiol 11, 1435-1443.

Fraser, D. W., Tsai, T.R., Orenstein, W., Parkin, W. E., Beecham, H. J., Sharrar, R. G., Harris, J., Mallison, G. F., Martin, S. M., \& other authors. (1977). Legionnaires' disease: description of an epidemic of pneumonia. N Engl J Med 297, 1189-1197.

Frech, C., Wunderlich, M., Glockshuber, R. \& Schmid, F. X. (1996). Preferential binding of an unfolded protein to DsbA. EMBOJ 15, 392-398.

Freedman, RB., Hint, T. O. R. \& Tuite, M. F. (1994). Protein disulphide-isomerase: building bridges in protein folding. TIBS 19, 331-336. 
Fronzes, R., Christie, P. J. \& Waksman, G. (2009a). The structural biology of type IV secretion systems. Nat Rev Micro 7, 703-714.

Gao, L. Y., Harb, O. S. \& Kwaik, Y. A. (1998). Identification of macrophage-specific infectivity loci (mil) of Legionella pneumophila that are not required for infectivity of protozoa. Infect Immun 66, 883-892.

Gao, L. Y. and Abu Kwaik, Y. (1999). Activation of caspase 3 during Legionella pneumophilainduced apoptosis. Infect Immun 67, 4886-4894.

Garduño, R. A., Quinn, F. D., \& Hoffman, P. S. (1998a). HeLa cells as a model to study the invasiveness and biology of Legionella pneumophila. Can J Microbiol 44, 430-400.

Garduño, R. A., Garduno, E., \& Hoffman, P. S. (1998b) Surface-associated Hsp60 chaperonin of Legionella pneumophila mediates invasion in a HeLa cell model. Infect Immun 66, 4602-4610.

Garduño, R. A., E. Garduño, M. Hiltz, \& P. S. Hoffman. (2002). Intracellular growth of Legionella pneumophila gives rise to a differentiated form dissimilar to stationary-phase forms. Infect Immun 70, 6273-683.

Gething, M. J. and Sambrook, J. (1992). Protein folding in the cell. Nature 355, 33-45.

George, J. R., L. Pine, M. W. Reeves, \& Harrell, W. K. (1980). Amino acid requirements of Legionella pneumophila. J Clin Microbiol 11, 286-291.

Glick, T. H., Gregg, M. B., Berman, B., Mallison, G. F., Rhodes, W. W. JR. \& Kassanoff, I. (1978). Pontiac fever: an epidemic of unknown etiology in a health department. I. Clinical and epidemiologic aspects. USA. J of Epidemiol 107, 149-160.

Graushof, U., Winther, J. K., Korber, P., Zander, T., Dallinger, P. \& Bardwell, J. C. A. (1995). Why is DsbA such an oxidizing disulfide catalyst? Cell 83, 947-955. 
Greer, P. W., Chandler, F. W. \& Hicklin, M. D. (1980). Rapid demonstration of Legionella pneumophila in unembedded tissue. An adaptation of the Giménez stain. Am J Clin Pathol 73, 788-790.

Greub, G. and D. Raoult. (2004). Microorganisms resistant to free-living amoeba. Clin Microbiol Rev 17, 413-433.

Grimshaw, J. P. A., Stirnimann, C. U., Brozzo, M. S., Malojcic, G., Grütter, M. G., Capitani, G. \& Glockshuber, R. (2008). DsbL and DsbI form a specific dithiol oxidase system for periplasmic arylsulfate sulfotransferase in uropathogenic Escherichia coli. J Mol Biol 380, 667-680.

Gross, C. A. (1996). Function and regulation of the heat shock proteins. Chap. 88 in Escherichia coli and Salmonella: cellular and molecular biology, American Society for Microbiology, Washington D.C., Neidhardt, Curtis III, Ingraham, Lin, Low, Magasanik, Reznikoff, Riley, Shaechter and Umbarger editors.

Guddat, L.W., Bardwell, J.C., Zander, TmAnd Martin, J. L. (1997). The uncharged surface features surrounding the active site of Escherichia coli DsbA are conserved and are implicated in peptide binding. Protein Sci 6, 1148-1156.

Guilhot, C., Jander, G., Martin, N. L. \& Beckwith, J. (1995). Evidence that the pathway of disulfide bond formation in Escherichia coli involves interactions between the cysteines of DsbB and DsbA. Proc Nat1 Acad Sci USA 92, 9895-9899.

Gupta, S. D., Wu, H. C., \& Rick, P. D. (1997). A Salmonella typhimurium genetic locus which confers copper tolerance on copper-sensitive mutants of Escherichia coli. J Bacteriol 179, 49774984. 
Haebel, P. W., Goldstone, D., Katzen, F., Beckwith, J., \& Metcalf, P. (2002). The disulfide bond isomerase DsbC is activated by an immunoglobulin-fold thiol oxidoreductase: crystal structure of the DsbC-DsbDalpha complex. EMBO J 21, 4774 - 4784.

Hailu, T. T., Foit, L., \& Bardwell, J. C. A. (2013). In vivo detection and quantification of chemicals that enhance protein stability. Anal Biochem. 434, 181-186.

Hatahet, F., Boyd, D. \& Beckwith, J. (2014). Disulfide bond formation in prokaryotes: History, diversity and design. Biochim Biophys Acta, http://dx.doi.org/10.1016/j.bbapap.2014.02.014.

Heinzen, R. A. (1997). Intracellular development of Coxiella burnetii. pp. 99-129. In: Rickettsial infection and immunity. Eds. Anderson, B., Bendinelli, M., and Friedman, H. Plenum Press, New York, N. Y.

Helsel, L. O., Bibb, W. F., Butler, C. A., Hoffman, P. S., \& McKinney, R. M. (1987). Recognition of a genus-wide antigen of Legionella by a monoclonal antibody. Curr Microbiol 16, 201-108.

Hendrix, L. R., Mallavia, L. P. \& Samuel, J. E. (1993). Cloning and sequencing of Coxiella burnetii outer membrane protein gene com1. Infect Immun 61, 470-477.

Heras, B., Kurz, M., Jarrott, R., Shouldice, S. R., Frei, P., Robin, G., Cemazar, M., ThönyMeyer, L., Glockshuber, R. \& Martin, J. L. (2008). Staphylococcus aureus DsbA does not have a destabilizing disulfide. A new paradigm for bacterial oxidative folding. J Biol Chem 283, 4261-4271.

Heras, B., Shouldice, S. R., Totsika, M., Scanlon, M. J., Schembri, M. A. \& Martin, J. L. (2009). DSB proteins and bacterial pathogenicity. Nat Rev Micro 7, 215-225.

Hiniker, A., Collet, J. F. \& Bardwell, J. C. A. (2005). Copper stress causes an in vivo requirement for the Escherichia coli disulfide isomerase DsbC. J Biol Chem 280, 33785-33791. 
Hoffman, P. S., Pine, L. \& Bell, S. (1983). Production of superoxide and hydrogen peroxide in medium used to culture Legionella pneumophila: catalytic decomposition by charcoal. Appl Environ Microbiol 45, 784-791.

Hoffman, P. S., Seyer, J. H. \& Butler, C. A. (1992). Molecular characterization of the 28- and 31-kilodalton subunits of the Legionella pneumophila major outer membrane protein. J Bacteriol $174,908-913$.

Holmgren A. (1979). Thioredoxin catalyzes the reduction of insulin disulfides by dithiothreitol and dihydrolipoamide. J Biol Chem. 254, 9627-9632.

Horwitz, M. A. (1983a). Cell-mediated immunity in Legionnaires' disease. J Clin Invest 71, 1686-97.

Horwitz., M. A. (1983b). Formation of a novel phagosome by the Legionnaires' disease bacterium (Legionella pneumophila) in human monocytes. $J$ Exp Med 158, 1319-31.

Hubber, A. and Roy, C. R. (2010). Modulation of host cell function by Legionella pneumophila type IV effectors. Annu Rev Cell Dev Biol 26, 261-283.

Hwang, C., Sinskey, A. J. \& Lodish, H. F. (1992). Oxidized redox state of glutathione in the endoplasmic reticulum. Science 257, 1496-1502.

Inaba, K., Takahashi, Y. Hei \& Ito, K. (2004). DsbB elicits a Red-shift of bound ubiquinone during the catalysis of DsbA oxidation. Journal of Biological Chemistry 279, 6761-6768.

Inaba, K., Murakami, S., Suzuki, M., Nakagawa, A., Yamashita, E., Okada, K. \& Ito, K. (2006). Crystal structure of the DsbB-DsbA complex reveals a mechanism of disulfide bond generation. Cell 127, 789-801. 
Inaba, K. (2010). MBSJ MCC Young Scientist Award 2009 REVIEW: Structural basis of protein disulfide bond generation in the cell. Genes to Cells 15, 935-943.

Ingmundson, A., Delprato, A., Lambright, D. G. \& Roy, C. R. (2007). Legionella pneumophila proteins that regulate Rab1 membrane cycling. Nature 450, 365-369.

Ishihara, T., Tomita, H., Hasegawa, Y., Tsukagoshi, N., Yamagata, H. \& Udaka, S. (1995). Cloning and characterization of the gene for a protein thiol-disulfide oxidoreductase in Bacillus brevis. J Bacteriol 177, 745-749.

Ito, K. \& Inaba, K. (2008). The disulfide bond formation (Dsb) system. Curr Opin Struct Biol $18,450-458$.

Jacob-Dubuisson, F., Pinkner, J., Xu, Z., Striker, R., Padmanhaban, A. \& Hultgren, S. J. (1994). PapD chaperone function in pilus biogenesis depends on oxidant and chaperone-like activities of DsbA. Proc Natl Acad Sci USA 91, 11552-11556.

Jameson-Lee, M., Garduno, R. A., \& Hoffman, P. S. (2011). DsbA2 (27 kDa DsbA2-like protein) of Legionella pneumophila catalyses extracytoplasmic disulfide-bond formation in proteins including the Dot/Icm type IV secretion system. Mol Microbiol 10, 1365-2958.

Jander, G., Martin, N. L. \& Beckwith, J. (1994). Two cysteines in each periplamic domain of the membrane protein DsbB are required for its function in protein disulfide bond formation. EMBOJ 13, 5121-5127.

Kadokura, H., Tian, H., Zander, T., Bardwell, J. C. A. \& Beckwith, J. (2004). Snapshots of DsbA in action: detection of proteins in the process of oxidative folding. Science 303, 534-537.

Kadokura, H. \& Beckwith, J. (2009). Detecting folding intermediates of a protein as it passes through the bacterial translocation channel. Cell 138, 1164-1173. 
Kadokura, H. \& Beckwith, J. (2010). Mechanisms of oxidative protein folding in the bacterial cell envelope. Antioxid Redox Signal 13, 1231-1246.

Kagan, J. C. and Roy, C. R. (2002). Legionella phagosomes intercept vesicular traffic from endoplasmic reticulum exit sites. Nat Cell Biol 4, 945-954.

Kamitani, S., Akiyama, Y. \& Ito, K. (1992). Identification and characterization of an Escherichia coli gene required for the formation of correctly folded alkaline phosphatase, a periplasmic enzyme. EMBO J 11, 57-62.

Katzen, F. \& Beckwith, J. (2000). Transmembrane electron transfer by the membrane protein DsbD occurs via a disulfide bond cascade. Cell 103, 769-779.

Katzen, F., Deshmukh, M., Daldal, F., \& Beckwith, J. (2002). Evolutionary domain fusion expanded the substrate specificity of the transmembrane electron transporter DsbD. EMBOJ 21, $3960-3969$.

Kaufmann, A. F., McDade, J. E., Patton, C. M., Bennett, J. V., Skaliy, P., Feeley, J. C., Anderson, D. C., Potter, M. E., Newhouse, V. F. \& others authors. (1981). Pontiac Fever: isolation of the etiologic agent (Legionella pneumophila) and demonstration of its mode of transmission. Am J Epidemiol 114, 337-347.

Kirby, J. E., Vogel, J. P., Andrews, H. L. \& Isberg, R. R. (1998). Evidence for pore-forming ability by Legionella pneumophila. Mol Microbiol 27, 323-336.

Kishigami, S., Akiyarna, Y. \& Ito, K. (1995). Redox States of DsbA in the periplasm of Escherichia coli. FEBS Lett 364, 55-58.

Kishigami, S. \& Ito, K. (1996). Roles of cysteine residues of DsbB in its activity to reoxidize DsbA, the protein disulphide bond catalyst of Escherichia coli. Genes Cells 1, 201-208. 
Kpadeh, Z. Z., Jameson-Lee, M., Yeh, A. J., Chertihin, O., Shumilin, I. A., Dey, R., Day, S. R., \& Hoffman, P. S. (2013). Disulfide bond oxidoreductase DsbA2 of Legionella pneumophila exhibits protein disulfide isomerase activity. J Bacteriol 195, 1825-33.

Lafaye, C., Iwema, T., Ferrer, J. L., Kroll, J. S., Griat, M., \& Serre, L. (2008). Preliminary crystallographic data of the three homologues of the thiol-disulfide oxidoreductase DsbA in Neisseria meningitidis. Acta Crystallogr Sect F Struct Biol Cryst Commun 64, 111-114.

Lafaye, C., Iwema, T., Carpentier, P., Jullian-Binard, C., Kroll, J. S., Collet, J. F., \& Serre, L. (2009). Biochemical and structural study of the homologues of the thiol-disulfide oxidoreductase DsbA in Neisseria meningitidis. J Mol Biol 392, 952-966.

Laguna, R. K., Creasey, E. A., Li, Z., Valtz, N. \& Isberg, R. R. (2006). A Legionella pneumophila translocated substrate that is required for growth within macrophages and protection from host cell death. Proc Natl Acad Sci USA 103, 18745-18750.

Lasica, A. M. \& Jagusztyn-Krynicka, E. K. (2007). The role of Dsb proteins of Gram-negative bacteria in the process of pathogenesis. FEMS Microbiol Rev 31, 626-636.

LeBlanc, J. J., Davidson, R. J. \& Hoffman, P. S. (2006). Compensatory functions of two alkyl hydroperoxide reductases in the oxidative defense system of Legionella pneumophila. J Bacteriol $188,6235-6244$.

Lee, J. V. and A. A. West. (1991). Survival and growth of Legionella species in the environment. J Appl Bacteriol symp sup 70, 121S-129S.

Leipelt, M., Schneidinger, B. \& Jaeger, K. E. (1997). Genbank accession U84726.

Lessi, M. and Lanka, E. (1994). Common mechanisms in bacterial conjugation and Ti-mediated T-DNA transfer to plant cells. Cell 77, 321-324. 
Liu, M., Conover, G. M., \& Isberg, R. R. (2008). Legionella pneumophila EhnC is required for efficient replication in tumor necrosis factor alpha-stimulated macrophages. Cell Microbiol 10, 1906-1923.

Luckey, M., Ling, R , Dose, A. \& Malloy, B. (1991). Role of a disulfide bond in the thermal stability of the LamB protein trimer in Escherichia coli outer membrane. J Bioi Chem 266, 18661871.

Luz, J. M. and Lennarz, W. J. (1996). Protein disulfide isomerase: a multifunctional protein of the endoplasmic reticulum. EYS 77, 97-117.

Machner, M. P. and Isberg, R. R. (2006). Targeting of host Rab GTPase function by the intravacuolar pathogen Legionella pneumophila. Dev Cell 11, 47-56.

Marra, A., S. J. Blander, M. A. Horwitz, \& H. A. Shuman. (1992). Identification of a Legionella pneumophila locus required for intracellular multiplication in human macrophages. Proc Natl Acad Sci USA 89, 9607-9611.

Marrie, T. J. (1993). Community-acquired Legionnaires disease: a reassessment, p. 46-47. In J. M. Barbaree, R. F. Breiman, and A. P. Dufour (ed.), Legionella: current status and emerging perspectives. American society for Microbiology, Washington, DC.

Marston, B. J., Lipman, H. B., \& Breiman, R. F. (1994). Surveillance for Legionnaires' disease: risk factors for morbility and mortality. Arch Intern Med 154, 2417-2422.

Martin, J. L., Bardwell, J. C. A. and Kuriyan, J. (1993). Crystal structure of the DsbA protein required for disulphide bond formation in vivo. Nature 365, 464-468.

McCusker, K. T., Braaten, B. A., Cho, M. W., \& Low, D. A. (1991). Legionella pneumophila inhibits protein synthesis in Chinese hamster ovary cells. Infect Immun 59, 240-246. 
McDade, J. E., Shepard, C. C., Fraser, D. W., Tsai, T. R., Redus, M. A. \& Dowdle, W. R. (1977). Legionnaires' disease: isolation of a bacterium and demonstration of its role in other respiratory disease. $N$ Engl J Med 297, 1197-1203.

Messens J., Collet, J. F., Van Belle, K., Brosens, E., Loris, R., \& Wyns, L. (2007). The oxidase DsbA folds a protein with a nonconsecutive disulfide. J Biol Chem 282, 31302-31307.

Metheringham, R., Griffiths, L., Crooke, H., Forsythe, S., \& Cole, J. (1995). An essential role for DsbA in cytochrome $c$ synthesis and formate-dependent nitrite reduction by Escherichia coli K-12. Arch Microbiol 164, 301-307.

Missiakas, D., Georgopoulos, C. \& Raina, S. (1993). Identification and characterization of the Escherichia coli gene $d s b B$, whose product is involved in the formation of disulfide bonds in vivo. Proc Nat1 Acad Sci USA 90, 7084-7088.

Missiakas, D., Georgopoulos, C. \& Raina, S. (1994). The Escherichia coli dsbC (xprA) gene encodes $a$ periplasmic protein involved in disulfide bond formation. EMBOJ 13, 2013-2020.

Missiakas, D., Schwager, F. \& Raina S. (1995). Identification and characterization of a new disulfide isomerase-like protein (DsbD) in Escherichia coli. EMBO J 14, 3415-3424.

Missiakas, D. and Raina, S. (1997a). Protein folding in the bactenal periplasm. J Bacteriol 179, 2465-2471.

Missiakas, D. and Raina, S. (1997b). Protein misfolding in the cell envelope of Escherichia coli: new signaling pathways. Trends Bioch Sci 22, 59-63.

Molofsky, A. B. and M. S. Swanson. (2004). Differentiate to thrive: lessons from the Legionella pneumophila life cycle. Mol Microbiol 53, 29-40. 
Morales, V. M., Bäckman, A. \& Bagdasarian, M. (1991). A series of wide-host-range lowcopy-number vectors that allow direct screening for recombinants. Gene 97, 39-47.

Morash, M. G., Brassinga, A. K., Warthan, M., Gourabathini, P., Garduno, R. A., Goodman, S. D., \& Hoffman, P. S. (2009). Reciprocal expression of integration host factor and $\mathrm{HU}$ in the developmental cycle and infectivity of Legionella pneumophila. Appl Environ Microbiol 75, 1826-1837.

Moulder, J. W., Hatch, T. P., Kuo, C. C., Schachter, J., \& J. Storz. (1984). Chlamydia. pp. 729-739. In: Bergey's Manual of Systematic Bacteriology. Eds. Kreig, R., and Holt, J. G. Williams and Wilkins, Baltimore, Maryland.

Muder, R. R. and Yu, V. L. (2002). Infection due to Legionella species other than $L$. pneumophila. Clin Infect Dis 35, 990-998.

Newton, H. J., Sansom, F. M., Dao, J., Cazalet, C., Bruggemann, H., Albert-Weissenberger, C., Buchrieser, C., Cianciotto, N. P., \& Hartland, E. L. (2008). Significant role for ladC in initiation of Legionella pneumophila infection. Infect Immun 76, 3075-3085.

Newton, H. J., Ang, D. K. Y., van Driel, I. R. \& Hartland, E. L. (2010). Molecular pathogenesis of infections caused by Legionella pneumophila. Clin Microbiol Rev 23, 274-298.

Ng, T. C., Kwik, J. F. \& Maier, R. J. (1997). Cloning and expression of the gene for a protein disulfide oxidoreductase from Azotobacter vinelandii: complementation of an Escherichia coli $d s b A$ mutant strain. Gen e188, 109-113.

Nguyen, M. H., J. E. Stout, \& Yu, V. L. (1991). Legionellosis. Infec Dis Clin North America 5, 561-584. 
Ngyen, T. M., D. Ilef, S. Jarraud, L. Rouil, C. Campese, D. Che, S. Haeghebaert, F. Ganiayre, F. Marcel, J. Etienne, \& Desenclos, J. C. (2006). A community-wide outbreak of Legionnaires disease linked to industrial cooling towers-how far can contaminated aerosols spread? J Infect Dis 193, 102-111.

Nosanchuk, J. D., and Casadevall, A. (2003). The contribution of melanin to microbial pathogenesis. Cell Microbiol 4, 203-223.

Okamoto, K, Baba, T., Yamanaka, H., Akashi, N. and Fujü, Y. (1995). Disulfide bond formation and secretion of Escherichia coli heat-stable enterotoxin II. J Bacteriol 177, 45794586.

Pan, X., Luhrmann, A., Satoh, A., Laskowski-Arce, M. A. \& Roy, C. R. (2008). Ankyrin repeat proteins comprise a diverse family of bacterial type IV effectors. Science 320, 1651-1654.

Pasculle, A. W., Feeley, J. C., Gibson, R. J., Cordes, L. G., Myerowitz, R. L., Patton, C. M., Gorman, G. W., Carmack, C. L., Ezzell, J. W. \& Dowling, J. N. (1980). Pittsburgh pneumonia agent: direct isolation from human lung tissue. J Infect Dis 141, 727-732.

Paumet, F., Wesolowski, J., Garcia-Diaz, A., Delevoye, C., Aulner, N., Shuman, H. A., Subtil, A. \& Rothman, J. E. (2009). Intracellular bacteria encode inhibitory SNARE-like proteins. PLoS ONE 4, e 7375.

Peek, J. A. and Taylor, R. K. (1992). Characterization of a periplasmic thiol:disulfide interchange protein required for the functional maturation of secreted virulence factors of Vibrio cholerae. Proc Natl Acad Sci USA 89, 6210-6214.

Philipps, B. and Glockshuber, R. (2002). Randomization of the entire active-site helix alpha 1 of the thiol-disulfide oxidoreductase DsbA from Escherichia coli. J Biol Chem 277, 43050-43057. 
Pine, L., Hoffman, P. S., Malcolm, G. B., Benson, R. F. \& Franzus, M. J. (1986). Role of keto acids and reduced-oxygen-scavenging enzymes in the growth of Legionella species. J Clin Microbiol 23, 33-42.

Qin, A., Scott, D. W., Rabideau, M. M., Moore, E. A., \& Mann, B. J. (2011). Requirement of the CXXC motif of novel Francisella infectivity potentiator protein B FipB, and FipA in virulence of F. tularensis subsp. tularensis. PLoS One 6, e24611.

Ren, G. and Bardwell, J. C. A. (2011). Engineered pathways for correct disulfide bond oxidation. Antioxid Redox Signal 14, 2399-2412.

Rietsch, A., Belin, D., Martin, N. \& Beckwith, J. (1996). An in vivo pathway for disulfide bond isomerization in Escherichia coli. Proc Natl Acad Sci USA 93, 13048-13053.

Rietsch, A., Bessette, P., Georgiou, G., \& Beckwith, J. (1997). Reduction of the periplasm disulfide bond isomerase, DsbC, occurs by passage of electrons from cytoplasmic thioredoxin. $J$ Bacteriol 179, 6602-6608.

Rogers, F. G., P. W. Greaves, A. D. Macrae, \& M. J. Lewis. (1980). Electron microscope evidence of flagella and pili on Legionella pneumophila. J Clin Pathol 33, 1184-1188.

Rogers, J., A. B. Dowsett, P. J. Dennis, J. V. Lee, \& C. W. Keevil. (1994). Influence of temperature and plumbing material selection on biofilm formation and growth of Legionella pneumophila in a model potable water system containing complex microbial flora. Appl Environ Microbiol 60, 1585-1592.

Rowbotham, T. J. (1980). Preliminary report on the pathogenicity of Legionella pneumophila for freshwater and soil amoebae. J Clin Pathol 33, 1179-1183.

Rowbotham, T. J. (1986). Current views on the relationships between amoebae, legionellae and man. Isr J Med Sci 22, 678-689. 
Roy, C. R., Berger, K. H. \& Isberg, R. R. (1998). Legionella pneumophila DotA protein is required for early phagosome trafficking decisions that occur within minutes of bacterial uptake. Mol Microbiol 28, 663-674.

Ruddock, L. W., Hirst, T. R \& Freeman, R. B. (1996). pH-dependance of the dithiol-oxidizing activity of DsbA (a periplasmic thiol:disulphide oxidoreductase) and protein disulphideisomerase: studies with a novel simple peptide substrate. Biochem J315, 1001-1005.

Ruiz, N., Chng, S. S., Hiniker, A., Kahne, D. \& Silhavy, T. J. (2010). Nonconsecutive disulfide bond formation in an essential integral outer membrane protein. Proc Natl Acad Sci USA 107, 12245-12250.

Sadoff, H. L. (1976). Encystment and germination in Azotobacter vinelandii. Bacteriol Rev 39, 516-524.

Sadosky, A. B. and Shuman, H. A. (1994). Genbank accession U15278.

Samuel, J. E., K. Kiss, \& Varghess, S. (2003). Molecular pathogenesis of Coxiella burnetii in a genomics era. Ann N Y Acad Sci 990, 653-663.

Segal, G., Purcell, M. \& Shuman, H. A. (1998). Host cell killing and bacterial conjugation require overlapping sets of genes within a 22-kb region of the Legionella pneumophila genome. Proc Natl Acad Sci USA 95, 1669-1674.

Segal, G. and H. A. (1999). Legionella pneumophila utilizes the same genes to multiply within Acanthamoeba castellanii and human macrophages. Infect Immun 67, 2117-2124.

Segatori, L., Paukstelis, P. J., Gilbert, H. F., Gerorgiu, G. (2004). Engineered DsbC chimeras catalyze both protein oxidation and disulfide-bond isomerization in Escherichia coli: reconciling two competing pathways. Proc Natl Acad Sci USA 101, 10018-10023. 
Segatori, L., Murphy, L., Arredondo, S., Kadokura, H., Gilbert, H., Beckwith, J., \& Georgiou, G. (2006). Conserved role of the linker alpha-helix of the bacterial disulfide isomerase DsbC in the avoidance of misoxidation by DsbB. J Biol Chem 281,4911-4919.

Seshadri, R., I. T. Paulsen, J. A. Eisen, T. D. Read, K. E. Nelson, W. C. Nelson, N. L. Ward, H. Tettelin, T. M. Davidsen, M. J. Beanan, R. T. Deboy, S. C. Daugherty, L. M. Brinkac, R. Madupu, R. J. Dodson, H. M. Khouri, K. H. Lee, H. A. Carty, D. Scanlan, R. A. Heinzen, H. A. Thompson, J. E. Samuel, C. M. Fraser, \& J. F. Heidelberg. (2003) Complete genome sequence of the Q-fever pathogen Coxiella burnetii. USA Proc Natl Acad Sci 100, 5455-5460.

Seshadri, R. and Samuel, J. (2005). Genome analysis of Coxiella burnetii species: insights into pathogenesis and evolution and implications for biodefense. Ann N Y Acad Sci 1063, 442-450.

Sevier, C. S. and Kaiser, C. A. (2008). Erol and redox homeostasis in the endoplasmic reticulum. Biochim Biophys Acta 1783, 549-556.

Sexton, J. A. and Vogel, J. P. (2002). Type IVb secretion by intracellular pathogens. Traffic 3, 178-185.

Sexton, J. A. and Vogel, J. P. (2004). Regulation of hypercompetence in Legionella pneumophila. J Bacteriol 186, 3814-3825.

Shevchik, V. E., Condemine, G. \& Robert-Baudouy, J. (1994). Characterization of DsbC, a periplasmic protein of Erwinia chrysanthemi and Escherichia coli with disulfide isomerase activity. EMBO J 13, 2007-2012.

Shevchik, V. E., Bortoli-German, I., Robert-Baudouy, J., Robinet, S., Barras, F. \& Condemine, G. (1995). Differential effect of $d s b A$ and $d s b C$ mutations on extracellular enzyme secretion in Erwinia chrysanthemi. Mol Microbiol 16, 745-753. 
Shouldice, S.R., Cho, S.H., Boyd, D., Heras, B., Eser, M., Beckwith, J., Riggs, P., Martin, J. L., \& Berkmen, M. (2010a). In vivo oxidative protein folding can be facilitated by oxidationreduction cycling. Mol Microbiol 75, 13-28.

Shouldice, S. R., Heras, B., Jarrott, R., Sharma, P., Scanlon, M. J., \& Martin, J. L. (2010b). Characterization of the DsbA oxidative folding catalyst from Pseudomonas aeruginosa reveals a highly oxidizing protein that binds small molecules. Antioxid Redox Signal 12, 921-931.

Shouldic, S. R., Heras, B., Walden, P. M., Totsika, M., Schembri, M. A., Martin, J. L. (2011). Structure and function of DsbA, a key bacterial oxidative folding catalyst. Antioxid Redox Signal 14, $1729-1760$.

Steel, T. W., J. Lanser, \& Sangster, N. (1990). Isolation of Legionella longbeacheae serogroup 1 from potting mixes. Appl Environ Microbiol 56, 49-53.

Steinert, M., L. Emody, R. Amann, \& Hacker, J. (1997). Resuscitation of viable but nonculturable Legionella pneumophila Philadelphia JR32 by Acanthamoeba castellanii. Appl Environ Microbiol 63, 2047-2053.

Steinert, M., U. Hentschel, \& Hacker, J. (2002). Legionella pneumophila: an aquatic microbe goes astray. FEMS Microbiol Rev 26, 149-162.

Stenson, T. H. \& Weiss, A. A. (2002). DsbA and DsbC are required for secretion of pertussis toxin by Bordetella pertussis. Infect Immun 70, 2297-2303.

Stirnimann, C. U., Rozhkova, A., Grauschopf, U., Grütter, M. G., Glockshuber, R., \& Capitani, G. (2005). Structural basis and kinetics of DsbD-dependent cytochrome $c$ maturation. Structure 13, 985-993.

Swanson, M. S. and Isberg, R. R. (1995). Association of Legionella pneumophila with the macrophage endoplasmic reticulum. Infect Immun 63, 3609-3620. 
Tan, Y. and Luo, Z. Q. (2011). Legionella pneumophila SidD is a deAMPylase that modifies Rab1. Nature 475, 506-509.

Tesh, M. J. and Miller, R. D. (1981). Amino acid requirements for Legionella pneumophila growth. J Clin Microbiol 13, 865-869.

Thomas, J. H. (1993). Thinking about genetic redundancy. Trends Genet 9, 395-399.

Thomas, V., T. Bouchez, V. Nicolas, S. Robert, J. F. Loret, \& Levi, Y. (2004). Amoebae in domestic water systems: resistance to disinfection treatments and implication in Legionella persistence. J Appl Microbiol 97, 950-963.

Tian, G., Xiang, S., Noiva, R., Lennarz, W., Schindelin, H. (2006). The crystal structure of yeast protein disulfide isomerase suggests cooperativity between its active sites. Cell 124, 61-73.

Tilney, L. G., Harb, O. S., Connelly, P. S., Robinson, C. G. \& Roy, C. R. (2001). How the parasitic bacterium Legionella pneumophila modifies its phagosome and transforms it into rough ER: implications for conversion of plasma membrane to the ER membrane. J Cell Sci 114, 46374650 .

Tinsley, C. R., Voulhoux, R., Beretti, J. L., Tommassen, J., \& Nassif, X. (2004). Three homologues, including two membrane-bound proteins, of the di- sulfide oxidoreductase DsbA in Neisseria meningitidis: effects on bacterial growth and biogenesis of functional type IV pili. $J$ Biol Chem 279, $27078-27087$.

Tison, D. L., D. H. Pope, W. B. Cherry, \& Fliermans, C. B. (1980). Growth of Legionella pneumophila in association with blue-green algae (Cyanobacteria). Appl Environ Microbiol 39, 256-259. 
Valster, R. M., Wullings, B. A. \& van der Kooij, D. (2010). Detection of protozoan hosts for Legionella pneumophila in engineered water systems by using a biofilm batch test. Appl Environ Microbiol 76, 7144-7153.

van Schaik, E. J., C. Chen, K. Mertens, M. M. Weber \& Samuel, J. E. (2013). Molecular pathogenesis of the obligate intracellular bacterium Coxiella burnetii. Nat Rev Microbiol 11, 561573.

Venetianer, P. and Straub, F. B. (1963). The enzymic reactivation of reduced ribonuclease. Biochim Biophys Acta 67, 166-168.

Vincent, C. D., Friedman, J. R., Jeong, K. C., Buford, E. C., Miller, J. L. \& Vogel, J. P. (2006). Identification of the core transmembrane complex of the Legionella Dot/Icm type IV secretion system. Mol Microbiol 62, 1278-1291.

Vivian, J. P., Scoullar, J., Rimmer, K., Bushell, S. R., Beddoe, T., Wilce, M. C. J., Byres, E., Boyle, T. P., Doak, B., \& other authors. (2009). Structure and Function of the Oxidoreductase DsbA1 from Neisseria meningitidis. Journal of Molecular Biology 394, 931-943.

Vogel, J. P., Andrews, H. L., Wong, S. K. \& Isberg, R. R. (1998). Conjugative transfer by the virulence system of Legionella pneumophila. Science 279, 873-876.

Wadowsky, R. M., R. B. Yee, L. Mezmer, E. J. Wing, \& Dowling, H. N. (1982). Hot water systems as sources of Legionella pneumophila in hospital and non-hospital plumbing fixtures. Appl. Environ. Microbiol 43, 1104-1110.

Wandersman, C. (1992). Secretion across the bacterial outer membrane. TIG 8, 317-322.

Watarai, M., Tobe, T., Yoshikawa, M. \& Sasakawa, C. (1995). Disulfide oxidoreductase activity of Shigella flexneri is required for release of Ipa proteins and invasion of epithelial cells. Proc Natl Acad Sci USA 92, 4927-4931. 
Winn, W. C., Jr. (1988). Legionnaires' disease: Historical perspective. Clin Microbiol Rev 150, 60-81.

Wunderlich, M. and Glockshuber, R. (1993) Redox properties of protein disulfide isomerase (DsbA) from Escherichia coli. Protein Sci 2, 717-726.

Yu, J., Webb, H. \& Hirst, T. R. (1992). A homologue of Escherichia coli DsbA protein involved in disulphide bond formation is required for enterotoxin biogenesis in Vibrio cholerae. Mol Microbiol 6, 1949-1958.

Yu, J., McLaughlin, S., Freedman, R. B. \& Hirst, T. R. (1993). Cloning and active site mutagenesis of Vibrio cholerae $d s b A$, a periplasmic enzyme that catalyzes disulfide bond formation. J Biol Chem 268, 4326-4330.

Yu, J. and Kroll, J. S. (1999). DsbA: a protein-folding catalyst contributing to bacterial virulence. Microbes Infect 1, 1221-1228.

Yu, V. L., Plouffe, J. F., Pastoris, M. C., Stout, J. E., Schousboe, M., Widmer, A., Summersgill, J., File, T., Heath, C. M., \& other authors. (2002). Distribution of Legionella species and serogroups isolated by culture in patients with sporadic community-acquired legionellosis: an international collaborative survey. J Infect Dis 186, 127-128.

Zapun, A., Bardwell, J. C. \& Creighton, T. E. (1993). The reactive and destabilizing disulfide bond of DsbA, a protein required for protein disulfide bond formation in vivo. Biochemistry 32, 5083-5092.

Zapun, A. and Creighton, T. E. (1994). Effects of DsbA on the disulfide folding of bovine pancreatic trypsin inhibitor and $\alpha$-lactalbumin. Biochemistry 33, 5202-5211. 
Zapun, A., Missiakas, D., Raina, S. \& Creighton, T.E. (1995). Structural and functional characterization of DsbC, a protein involved in disulfide bond formation in Escherichia coli. Biochemistry 34, 5075-5089.

Zhang, H. Z. and Donnenberg, M. S. (1996). DsbA is required for stability of the type IV pilin of enteropathogenic Escherichia coli. Mol Microbiol 21, 787-797.

Zheng, W. D., Quan, H., Song, J. L., Yang, S. L. \& Wang, C. C. (1997). Does DsbA have chaperone-like activity? Arch Biochem Biophys 337, 326-331. 